\title{
Neugeborenen-, Hunger- und Intoxikationsacidosis in ihren Beziehungen zueinander.
}

\author{
Studien über Acidosis bei Säuglingen, insbesondere im Lichte des \\ Wasserstoffionen-,,Stoff wechsels". \\ Von \\ Arvo Ylppö.
}

(Aus dem Kaiserin Auguste Victoria Hause zur Bekämpfung der Säuglingssterblichkeit im Deutschen Reiche, Charlottenburg [Dir.: Prof. Dr. Langstein].)

Mit 34 Textfiguren.

(Eingegangen am 1. Juli 1916.)

Inhaltsverzeichnis.

I. Einleitung (S. 269).

II. Geschichtliche Entwicklung der Acidosefrage (S. 270).

III. Methoden.

A. Die Bestimmung der Acidität oder der wahren Reaktion (S. 282).

1. Die wahre Reaktion des Urins (S. 283).

2. Die wahre Reaktion des Stuhls (S. 283).

3. Die wahre Reaktion des Blutes (S. 283).

4. Die wahre Reaktion der Gewebe einschließlich des Magen. und Darminhalts (S. 284).

B. Die Bestimmung der $\mathrm{CO}_{2}$-Regulationsbreite (S. 285).

1. Kritik der Methode (S. 287).

C. Die Dissoziationskurve des Oxyhämoglobins im Blnte (S. 290).

IV. Untersuchungen an jungen Säuglingen (Frühgeborenen und Neugeborenen).

A. Die wahre Reaktion des Urins und des Stuhls bei steigendem Alter und bei verschiedener Ernährung (S. 293).

1. Urin allein (S. 293).

2. Urin und Stuhl bei Frauenmilch- und bei Kuhmilchernährung (S. 296).

3. Einfluß der Acidität der Nahrung auf die wahre Reaktion des Urirs (S. 300).

B. Die $\mathrm{CO}_{2}$-Regulationsbreite des Blutes mit zunehmendem Alter (S. 303).

C. Die $\mathrm{O}_{2}$-Dissoziationskurve des Blutes mit zunehmendem Alter (S. 305).

D. Die Reaktion der Gewebe und des Herzblutes cinschließlich der Bestimmung der $\mathrm{CO}_{2}$-Regulationsbreite und der Dissoziationskurven bei Frühgeborenen und jungen Süuglingen (S. 308). 
Arvo Ylppö: Neugeborenen-, Hunger- und Intoxikationsacidosis usw. 269

V. Untersuchungen im Hunger.

A. Die Einwirkung des Hungers auf den Säuglingsorganismus im Sinne der "Acidose" (Untersuchungen wie A-D Kapitel IV) (S. 312).

B. Die Einwirkung des Hungers auf den Tierorganismus (S. 320).

VI. "Acidose". Untersuchungen bei Intoxikation. (S. 322).

A. Die wahre Reaktion des Urins und des Stuhls bei Intoxikation (S. 322).

B. Die Reaktion und die $\mathrm{CO}_{2} \cdot$ Regulationsbreite des Blutes bei Intoxikation (S. 323).

C. Die $\mathrm{O}_{2}$-Dissoziationskurve des Blutes bei Intoxikation (S. 326).

D. Die Reaktion der Gewebe bei Intoxikation (S. 332).

E. Die Reaktion des Magen- und Darminhalts bei Intoxikation (S. 338).

VII. Therapeutisches zur Bekämpfung der Acidosebei Intoxikation (S. 339).

VIIL. Besprechung der Ergebnisse mit Berücksichtigung der Klinik (S. 345).

IX. Zusammenfassung (S. 362).

$X$. Literaturverzeichnis speziell über Intoxikation und die Acidosefrage (S. 367).

XI. Protokolle mit Krankengeschichten (S. 374).

\section{Einleitung.}

Schon seit langem spielt die sogenannte Säurevergift ung als Ursache der verschiedensten Störungen im Kindesalter eine große Rolle. Aber erst durch die großen Fortschritte der physiologisch-chemischen Forschung am Ende des 19. Jahrhunderts bekam diese alte Lehre eine feste Grundlage. Nachdem Czerny und Keller, auf diesen neueren Anschauungen aufbauend, ihre Säureintoxikationshypothese der chronisch magendarmkranken Säuglinge aufgestellt haben, wurde die Frage in der Pädiatrie aktuell und gab Anlaß zu einer großen Reihe von Untersuchungen, die mehr oder minder direkt die Säurevergiftungsoder, wie sie nunmehr heißt, Acidosefrage berührten. Es wurde lebhaft für und wider diese neue Hypothese gestritten. In den ersten Jahren dieses Säculums war der Kampf besonders lebhaft, bis er dann allmählich nachließ, noch ehe eine volle Einigkeit erzielt worden. war.

Die Acidosefrage selbst hat seither durch neue biologische Methoden fortwährend eine durchgreifende Vertiefung erfahren. Aus diesem Umstand leitete ich die Berechtigung her, die Acidosefrage im Säuglingsalter nochmals aufzurollen.

Die leitenden Gedanken bei der nachfolgenden Arbeit will ich hier vorweg kurz skizzieren: 
I. Zunächst stellte ich mir zur Aufgabe, nach acidotischen Merkmalen im Organismus des gesunden, jungen Säuglings (neugeborenen oder frühgeborenen) zu fahnden. Dies mit Rücksicht auf die bekannte Tatsache, daß die Intoxikation am häufigsten bei jungen Säuglingen auftritt. Und die Intoxikation gilt doch ihrer Ähnlichkeit wegen mit dem Coma diabeticum, mit einem sicher acidotischen Zustande, als ein Paradigma der Acidose im Säuglingsalter.

II. Wollte ich bei jungen Säuglingen während des Hu ngers nach den gleichen Prinzipien nach acidotischen Merkmalen forschen. Die neueren Untersuchungen haben ja festgestellt, daß der Hungerzustand ein gewissermaßen acidotischer Zustand ist.

III. Wollte ich die Paradigma-Fälle, d. h. die Intoxikationen selbst, von dem gleichen Standpunkte aus untersuchen.

Zur Feststellung eines acidotischen Zustandes habe ich folgende Wege benutzt:

Ich habe in den drei erwähnten Gruppen

1. den ganzen Wasserstof fio ne $\mathrm{n}-,, \mathrm{S}$ to ff wechsel" verfolgt, $d, \mathrm{~h}$. ich habe unter Berücksichtigung der jeweiligen Nahrung die wahre Reaktion des Blutes, des Urins und Stuhls in kürzeren oder längeren Perioden systematisch festgestellt;

2. die Kohlensäure-Regulationsbreite des Blutes bestimmt, die ja nach den folgenden Ausführungen ein empfindlicher Indikator für die acidotischen Zustände ist;

3. die Saverstoff-Bindungsverhältnisse des Blutes mit Hilfe der Oxyhämoglobin-Dissoziationskurve bei den in Betracht kommenden Kindern systematisch bestimmt;

4. die wahre Reaktion der verschiedensten Gewebe (Muskulatur, Gehirn, Leber, Milz usw.) sowohl bei Intoxikationen als auch bei an anderen Krankheiten verstorbenen Kindern (darunter Frühgeborene, Neugeborene und ältere Säuglinge) untersucht.

Auf diese Weise hoffte ich einen Einblick, nicht nur in die bei den erwähnten Zuständen möglicherweise eintretenden Verschiebungen im Basen- und Säurengleichgewicht, sondern auch in die Veränderungen bezüglich der oxydativen und respiratorischen Funktionen des Organismus zu bekommen.

\section{Gesehichtliche Entwicklung der Acidosefrage.}

Bevor ich auf die Untersuchungen näher eingehe, lasse ich eine Ubersicht über die geschichtliche Entwicklung der Acidosefrage vorangehen. 
Fleis $\mathrm{ch}^{1}$ ) hat meines Wissens als erster im Jahre 1803 die Atrop hie der Säuglinge auf eine chronische $S$ ä urevergift ung zurückgeführt. In dem Kapitel „Darrsucht oder Auszehrung der Kinder“, S. 529 seines „Handbuches über die Krankheiten der Kinder", gibt er zunächst an, daß „allzuwässrige und vegeta bilische Nahrungsmittel" säureerzeugend wirken und daß diese Säuren ,in den ersten Wegen" eine sehr wichtige Ursache der Atrophie sind. Weiterhin sagt er wörtlich: „Der Speisebrey wird hierdurch verdorben und wahrhaft sauer, so wie auch die beste Galle die Verdauung nicht bewirken kann, denn sie wird durch die Säure praezipidirt. - Die nicht durch die Galle bezwungene Säure aber wird der Ausscheidung des Milchsafts nachtheilig werden, das minder genährte Kind wird immer mehr geschwächt, es wird also nach und nach sich die Krankheit einschleichen und langsam aber täglich zunehmen." Interessant ist ferner, wie er in der Alkalitherapie das wirksamste Mittel gegen Atrophie sah. S. 539 schreibt er: „Zur Tilgung der Säure dienen erdige, laugenhafte und bittere Mittel als: Magnesia alba, Kalchwasser" usw., zum Schluß noch Seife und eingedickte Ochsengalle. Fleisch kannte auch schon gut den Zustand, den wir jetzt Intoxikation der Säuglinge nennen, wie es aus seiner diesbezüglichen Beschreibung S. 512 hervorgeht: Als Beweis dafür mögen seine folgenden Sätze sprechen: ,Die Augen sind matt und eingefallen und die Kinder schließen solche im Schlafe gemeiniglich nur halb zu. Zuweilen ist bey dieser Krankheit bey einigen Patienten eine so große Unempfindlichkeit vorhanden, daß sich die Fliegen in das offene Auge setzen, ohne daß die Kinder mit den Augenliedern die geringste Bewegung machen, solche fortzujagen." Er hat aber keine Ursache für diese Krankheit angeben können, hat sie auch in keinen Zusammenhang mit der Säurevergiftung gebracht. Dies hat zum ersten Male Ja eger $\left.{ }^{2}\right) 1813$ getan. Er beschreibt unter dem Namen ,akute Magenerweichung“ ein Krankheitsbild, das identisch ist mit dem heutigen Bilde der Intoxikation. $\mathrm{Zu}$ der eigentümlichen Bezeichnung führten ihn seine Beobachtungen am Sektionstisch (Erweichung der Magenwände, die durch spätere Forschungen als postmortale Erscheinung nachgewiesen worden sind) und die damaligen Anschauungen, die den Verdauungstraktus (Magen und Darm) als ein einheitliches Ganzes betrachteten. Seine ,,akute Magenerweichung“",

1) Fleisoh, Handbuch über die Krankheiten der Kinder. Leipzig 1803 (F. G. Jacobäer), 1. Bd.

2) Jaeger, zit. nach Barthez et Rilliet, Handb. d. Kinderkrankheiten. Deutsche Ausgabe von Dr. Hagen. Leipzig 1855, Teil I, 701.

Zeitschrift flir Kinderheilkunde. O. XIV. 
die, wie er anführt, oft mit Gehirnaffektionen kompliziert ist, betrachtet er als Folge von Vermehrung der Essigsäure, die zerstörend auf die Magenwand wirke.

Aus diesen vagen Vermutungen kam man erst gegen Ende des 19. Jahrhunderts dank der neubelebten experimentellen Forschung hinaus. Den ersten Anstoß gaben die Untersuchungen von Walter ${ }^{1}$ ). Er fand 1877 bei Tieren nach Zufuhr von anorganischen Säuren (Salz- und Phosphorsäure) im Urin größere Mengen von Ammoniak und erklärte die Vermehrung des Ammoniaks als Abwehrmittel des Organismus gegen Säurevergiftung. Hallervorde $n^{2}$ ) fand dann 1880 bei Diabetikern im Urin ebenfalls einen vermehrten Gehalt an Ammoniak und glaubte sich berechtigt, in Analogie zu den Walterschen Versuchen, diesen auf abnorme im Körper zirkulierende Säuren zurückzuführen. Er hatte außerdem im Diabetikerurin eine vermehrte $\mathrm{P}_{2} \mathrm{O}_{5}$ Ausscheidung gefunden und glaubte aus diesem Grunde; daß die supponierten Säuren anorganischer Natur seien, hielt aber daneben auch das Vorhandensein von organischen Säuren wie Milchsäure $\mathbf{u}$. dgl. für möglich. 1883 erschien eine Arbeit von Stadelman $n^{3}$ ), worin er die Entdeckung einer neuen Säure im Diabetikerurin anzeigte, die er mit $\beta$-Crotonsäure identifizieren wollte. Im folgenden Jahre zeigten dann aber kurz nacheinander $K \ddot{u} l z^{4}$ ) und Minkowski ${ }^{5}$ ), daß es sich in der Tat nicht um $\beta$-Crotonsäure, sondern um $\beta$-Oxybuttersäure handelte. Diese kommt in großer Menge im Diabetikerurin vor. Es kam noch dazu, daß die große Atmung beim Coma diabeticum, wie sie Kußma ul $\left.{ }^{6}\right) 1874$ so klassisch beschrieben hatte, eine auffallende Ähnlichkeit mit der Atmung bei mit Säure vergifteten Tieren zeigte.

1) Walter, Fr., Untersuchungen über die Wirkung der Säuren auf den thierischen Organismus. Archiv f. experim. Pathol. u. Pharmakol. 7, 148. 1877.

2) Hallervorden, Utber Aussoheidung von Ammoniak im Urin bei patho. logischen Zuständen. Archiv f. experim. Pathol. u. Pharmakol. 12, 237. 1880 ,

3) Stadel mann, Über die Ursachen der pathologischen Ammoniakausscheidung beim Diabetes mellitus und des Coma diabeticum. Archiv f. experim. Pathol. u. Pharmakol. 11, 419. 1883.

4) Külz, Über eine neue linksdrehende Säure (Pseudooxybuttersäure). Ein Beitrag zur Kenntnis der Zuckerruhr. Zeitschr. f. Biol. 20, 165. 1884.

5) Minkowski, Uber das Vorkommen von Oxybuttersäure im Harn bei Diabetes mellitus. Ein Beitrag zur Lehre von Coma diabeticum. Archiv f. experim. Pathol. u. Pharmakol. 18, 35. 1884.

6) KuBmaul, Zur Lehre vom Diabetes mellitus. Dentsches Archiv f. klin. Med. 14, 1. 1874. 
Auf Grund des Obigen hielt man für festgelegt, daß es sich beim Coma diabeticum um einen Zustand der Säurevergiftung handelte, einen Zustand, den zuerst Na un $\mathbf{y}^{\mathbf{1}}$ ) mit dem Namen „Acidosis“" bezeichnete. Genauer gesagt meinte Naunyn mit Acidosis einen Zustand, bei dem Salze unverbrennender organischer Säuren in ungewöhnlicher Menge im Blute kreisen. Auf diese Definition möchte ich wegen der oft falschen Auffassung über den ursprünglichen Begriff der Acidosis schon hier besonders aufmerksam machen.

1897 wurde dieser Acidosisbegriff zum ersten Male in die Paediatrie übernommen. Den Stein ins Rollen brachte Keller ${ }^{2}$ ) mit seiner Arbeit über Ammoniakausscheidung. Er zeigte darin, daß bei chronisch magendarmkranken Säuglingen die Menge des ausgeschiedenen Ammoniaks sowohl absolut als auch im Verhältnis zum Gesamtstickstoff bedeutend vermehrt war. Als Erklärung könnte nach ihm entweder eine Vermehrung der im Blute zirkulierenden Säuren oder eine Störung in der Harnstoffsynthese oder die Kombination beider Faktoren in Frage kommen. Bei der näheren Analyse dieser Faktoren konnte Keller zeigen, daß die chronisch magendarmkranken Säuglinge sowohl verschiedene Aminosäuren (Glykokoll, Leucin, Asparagin) ${ }^{3}$ ) wie Ammoniaksalze ${ }^{4}$ ) normalerweise in ihrem Körper in Harnstoff überführen konnten. Damit mußte man nach Keller die Störung in der Harnstoffsynthese als ursächlicher Faktor bei der vermehrten Ammoniakausscheidung außer Betracht lassen. Bei Erwachsenen und im Tierexperiment hatte man beobachtet, daß bei Alkalizufuhr die Ammoniakmenge des Harns zurückgeht. In der zurückgehenden Menge, die man als Neutralisator der überschüssigen Säuren betrachtete, sah Münzer ${ }^{5}$ ) ein Kriterium für die Acidosis. An der Hand dieses sog. Schröder-Mü nzerschen Kriteriums glaubte dann van den $\operatorname{Bergh}^{6}$ ) einen schlagenden

1) Naunyn, Der Diabetes mellitus. 2. Auflage. Wien 1906 (Hölder).

2) Keller, Zur Kenntnis der Gastroenteritis im Säuglingsalter. Mitt. II: Ammoniakausscheidung. Jahresber. f. Kinderheilk. 44, 25. 1897.

3) Keller, Das Schicksal der Amidosäuren im Organismus des magendarmkranken Säuglings. Centralbl. f. allg. Pathol. u. pathol. Anat. 9, 739.1898.

4) Keller, Einfluß der Zufuhr von Ammoniaksalzen auf die Harnstoffausscheidung. Jahrb. f. Kinderheilk. 47, 187. 1898.

5) Münzer, Die harnstoffbildende Funktion der Leber. Arehiv f. experim. Pathol. u. Pharmakol. 33, 164. 1894. (Daselbst S. 193.)

6) van den Bergh, Zur Kenntniss der Gastroenteritis im Säuglingsalter. III. Mitt. : Einfluß von Alkalizufuhr auf die $\mathrm{NH}_{3}$-Ausscheidung. Jahrb. f. Kinderheilk. 45, 265. 1897. 
Beweis dafür bringen zu können, daß die vermehrte Ammoniakausscheidung im Urin chronisch magendarmkranker Säuglinge tatsächlich auf vermehrte Säurebildung (Acidosis) bei denselben Kindern zurückzuführen sei. Er sah nämlich bei Kindern, die 2-5 g Natrium bicarbonicum täglich bekamen, die Ammoniakmenge des Urins stark zurückgehen, in einzelnen Fällen sogar ganz verschwinden. Dazu kam noch, daß Czern $y^{1}$ ) eine auffallende Äbnlichkeit in der Respirationskurve und im ganzen Respirationstypus bei schwerkranken, im Sterben liegenden magendarmkranken Säuglingen und bei säurevergifteten Tieren fand. Auf Grund der obigen Befunde hielten nun Czerny und $\mathrm{Keller}^{2}$ ) sich berechtigt, in der Säurevergiftung chronisch magendarmkranker Säuglinge die Ursache der Atrophie zu suchen, und hiermit war die Czerny - Kellersche Säureintoxikations-Hypothese entstanden.

Diese Hypothese bildete den Ausgangspunkt einer großen Anzahl von Arbeiten der folgenden Jahre und hat dadurch ungemein befruchtend auf unsere Kenntnisse, nicht nur über chronische Verdauungsstörungen, sondern auch über andere Fragen aus dem Gebiete der Pathologie des Stoffwechsels im Säuglingsalter gewirkt.

Beim Versuch, die Hypothese zu stützen, stieß man auf Widersprüche. Zunächst konnte $\mathrm{Keller}^{3}$ ) nicht nachweisen, daß̣ im Urin bei chronisch magendarmkranken Säuglingen tatsächlich größere Mengen von organischen Säuren ausgeschieden werden, wie die ursprüngliche Hypothese voraussetzte. In früheren Arbeiten hatten Schrack ${ }^{4}$ ) und Baginsky ${ }^{5}$ ) keine Vermehrung der Acetonkörper im Urin chronisch magendarmkranker Säuglinge gefunden. Auch fand Keller durch die Freund-Liebleinsche Methode (die die Acidität durch das prozentuelle Verhältnis von zweifach saurer Phosphorsäure zur Gesamtphosphorsäure angibt) keine besondere Steigerung der Harnacidität bei magendarmkranken Kindern. Weiterhin haben

1) Czerny, Zur Kenntnis der Gastroenteritis im Säuglingsalter. IV. Mitt.: Respirationsstörungen. Jahrb. f. Kinderbeilk. 45, 271. 1897.

2) Siehe 1 u. flg., außerdem: Des Kindes Ernährung, Ernährungsstörungen und Ernährungstherapie. II. Bd., S. 135-189. Leipzig (Deuticke).

9) Diese Versuche von Keller sind nicht veröffentlicht worden, sie werden aber von der Breslauer Schule in obigem Sinne durch Freund und Steinitz zitiert. (Stei nitz, Monatsschr. f. Kinderheilk. 1, 227. 1902. - Freund, ibidem S. 231.)

4) Schrack $k$, Über Acetonurie und Diaceturie bei Kindern. Jahrb. f. Kinderheilk. 29, 4l1. 1889.

5) Baginsky, Über Acetonurie bei Kindern. Archiv f. Kinderheilk. 9, 1. 1888. 
Czerny und Keller ${ }^{1}$ ) nachgewiesen, daB die Vermehrung des Ammoniakgehaltes in außerordentlich hohem Grade vom Fettgehalt der Nahrung abhängig ist, und durch diesen Befund wurde der Wert der Ammoniakvermehrung als Ausdruck von im Körper im therschuß gebildeten Säuren stark beeinträchtigt.

Es meldeten sich auch allmählich die Stimmen, die die Vermehrung der Ammoniakausscheidung im Urin entweder ganz und gar oder teilweise auf Störung der normalen Oxydation von Ammoniak zu Harnstoff bei chronisch magendarmkranken Kindern zurückführen wollten. Freund ${ }^{2}$ ) glaubte als erster zeigen zu können, daß bei atrophischen Kindern eine gewisse Schwäche der oxydativen Funktionen vorhanden sei, indem er bei Atrophie verminderte Oxydation von Benzol in Phenol fand.

In noch bestimmterer Form trat Pfaundler ${ }^{3}$ ) für diese Meinung ein. In einer besonders wertvollen und sorgfältigen Arbeit aus dem Jahre 1901 unterzieht er alle oben angeführten Argumente für die Czern y - Kellersche Intoxikationshypothese einer eingehenden Kritik. Er zeigt zunächst, daß die Versuche von van den Bergh und das ganze Schröder - Münzersche Kriterium keineswegs imstande sind zu beweisen, daß der Ammoniakteil, der bei Alkalizufuhr aus dem Urin verschwindet, einfach als Neutralisator der Säuren angesehen werden darf. Erstens geht, wie schon aus den Stadel mannschen ${ }^{4}$ ) und auch aus van den Berghs eigenen Versuchen hervorgeht, auch die Gesamtstickstoffausscheidung bei Alkalizufuhr stark zurück, was damit zusammenhängt, daß künstlich zugeführte Alkalimengen bisher noch ganz unbekannte Umwälzungen im Stickstoffwechsel verursachen. Zweitens weist er nach, daß in einzelnen Versuchen von va n de $n$ Bergh der Urin alkalisch war, wobei einfach aus physikalisch-chemischen Gründen die Aussoheidung von Ammoniak unmöglich ist. Weiterhin zeigt Pfaundler, daß chronisch magendarmkranke Kinder nicht größere Mengen Ammoniak als gesunde Kinder bei der gleichen Ernährung ausscheiden. Nach ihm ist die renale Ammoniakausscheidung im Säuglingsalter erhöht, einesteils bei vermehrtem Fettgehalt der

1) Czerny u. Keller, Zur Kenntnis der Gastroenteritis im Säuglingsalter. V. Mitt.: Säurebildung. Jahrb, f, Kinderheilk. 45, 274. 1897.

2) Freund, Zur Kenntnis der Oxydationsvorgänge bei gesunden und kranken Säuglingen. Verhandl. d. Gesellsch. f. Kinderheilk., Hamburg 1901, S. 187.

3) Pfaundler, Über Stoffwechselstörungen bei magendarmkranken Säuglingen. Jahrb. f. Kinderheilk. 54, 247. 1901.

4) Stadelmann, Über den Einfluß der Alkalien auf den menschlichen Stoffwechsel. Stuttgart 1890. 
Nahrung, andernteils wenn eine Erkrankung des Leberparenchyms, z. B. höhergradige fettige Entartung, vorliegt. Und drittens, wenn aus irgendwelchen Gründen ein schwerster Allgemeinzustand begleitet von Zirkulations- und Respirationsstörungen vorliegt, bleibt es in gleicher Weise einerlei, ob das Kind an einer Ernährungsstörung gelitten hat oder nicht. Daß bei Erkrankungen des Leberparenchyms eine Oxydationsstörung der Leber vorhanden ist, versuchte er dadurch nachzuweisen, daß er Leberbrei auf Salicylaldehyd eine bestimmte Zeit wirken ließ und die Menge der durch die Oxydation entstandenen Salicylsäure feststellte. Er fand die größten Salicylsäuremengen bei Säuglingen, die makroskopische Leberveränderungen bei der Sektion zeigten und die im Leben den höchsten $\mathrm{NH}_{3}$-Koeffizienten hatten. Thie mich ${ }^{1}$ ) hatte schon vorher gezeigt, daß die meisten an Magendarmkrankheiten verstorbenen Säuglinge geringere oder größere Degenerationserscheinungen der Leber hatten. Es lag demnach der Gedanke nahe, daß die vermehrte Ammoniakausscheidung bei magendarmkranken Kindern auf die verminderte Oxydationsfähigkeit der geschädigten Leber bei den betreffenden Kindern zurückzuführen sei, ohne daß irgend welche Säurevergiftung zu bestehen brauchte. Die Angaben über die $\mathrm{Oxy}$ dationsstörung in der Leber von Pfaundler wurden dann weiterhin an der Hand derselben Methode von Brüning ${ }^{2}$ ) bestätigt.

Dadurch schien die Czerny-Kellersche Säurevergiftungs-Hypothese schwer erschüttert zu sein. Als Retter trat Steinit $\left.z^{3}\right)^{4}$ ) auf. Keller hatte schon in seiner "Malzsuppe"5) den Gedanken ausgesprochen, daß vielleicht die Acidose und die darauf folgende gesteigerte Ammoniakausscheidung auch dadurch hervorgerufen sein könnte, daß zu wenig Alkali in den Organismus eingeführt resp. aus dem Darm resorbiert würde. Und er hat sich auch in einer speziellen Arbeit ${ }^{6}$ )

1) Thiemich, Über Leberdegeneration bei Gastroenteritis. Zieglers Beiträge z. pathol. Anat. u. z. allg. Path, 20, 179. 1896.

2) Brüning, Über die Beziehungen zwischen Lebererkrankungen und postmortaler Oxydationskraft des Lebergewebes. Monatsschr. f. Kinderheilk. 2, 129. 1903.

3) Steinitz, Zur Kenntnis der chronischen Ernährungsstörungen der Säuglinge. I. Mitt.: Alkalistoffwechsel. Monatsschr. f. Kinderheilk. 1, 225. 1902.

4) Steinitz, Zur Kenntnis der chronischen Ernährungsstörungen der Säuglinge. Jahrb. f. Kinderheilk. 5\%, 689. 1903.

5) Keller, Malzsuppe, eine Nahrung für magendarmkranke Säuglinge. Jena 1898 (Gustav Fischer).

8) Keller, Zur Kenntnis der chronischen Ernährungsstörungen der Säuglinge. III. Fettumsatz und Acidose. Monatsschr. f. Kinderheilk. 1, 234. 1902. 
näher mit dieser Frage beschäftigt. Keller ging von dem Gedanken aus, daß das Fett der Nahrung im Magen- und Darmkanal durch Verseifung Alkali, Kalk und Magnesia bindet und durch Nichtresorption dem Organismus Basen entzieht, die sonst in den Kreislauf gelangen und hier saure Stoffwechselprodukte neutralisieren würden. Auf diese Weise könnte ein Alkalidefizit, eine relative Säuerung des Organismus, und infolgedessen eine gesteigerte Ammoniakausscheidung zustande kommen. Seine diesbezüglichen Versuche hatten aber ein negatives Ergebnis. Bei Fettanreicherung der Nahrung wurde der Gehalt der Faecestrockensubstanz an Ätherextrakt nicht wesentlich verändert. Das Verhältnis von Neutralfett plus freien Fettsäuren zu den Seifen änderte sich auch keineswegs konstant so, daß man auf diesem Wege keine vermehrte Alkaliausscheidung feststellen konnte. Dieses ist erst S teinitz gelungen in seinen ähnlich angestellten Versuchen, in welchen er bei 4 Kindern bei fettreicher Nahrung (bis 6\% Fett) den gesamten Alkalistoffwechsel verfolgte und dabei eine starke Vermehrung der durch den Darm ausgeschiedenen Alkalien und gleichzeitige Vermehrung des Ammoniaks im Urin feststellte. Auf Grund dieser Versuche wurde die Säurevergiftungs-Hypothese dahin formuliert, daß es sich bei ehronisch magendarmkranken Kindern nicht um eine echte Acidose handelt, mit vermehrter Produktion von Säuren, sondern um eine „relative Acidose" mit dem Hauptmerkmal einer gewissen Alkaliarmut des Organismus und infolgedessen einer Hyperproduktion von Ammoniak zur Neutralisation der in normalen Grenzen gebildeten Säuren.

Alle bisherigen Arbeiten versuchten lediglich durch Urin- und Stuhluntersuchungen Schlüsse auf die Acidose zu ziehen. Pfa u ndler ${ }^{1}{ }^{2}$ ) hat zuerst 1904 versucht, die Verhältnisse im Blute selbst klarzulegen. Er bestimmte bei einer Anzahl von Kindern, in der Mehrzahl der Fälle im Leichenblute, bei wenigen im Leben, die $\mathrm{OH}-$ Ionenkonzentration, konnte aber dabei im Blute von atrophischen und chronisch magendarmkranken Kindern keine konstanten Werte festlegen, die die Czern yKellersche Säurevergiftungslehre hätten stützen können. Nur bei Frühgeburten konnte er im Herzblute konstant erhöhte Acidität feststellen.

1) Pfaundler, Physikalisch-chemische Untersuchungen an Kinderblut. Verhandl. d. Gesellsch. f. Kinderheilk., Breslau 1904, S. 24, und Jahrb. f. Kinderheilk. 60, 719. 1904.

2) Pfaundler, UUber die aktuelle Reaktion des kindlichen Blutes. Archiv f. Kinderheilk. 41, 161. - 1905. 
Der „,chronisch magendarmkranke Säugling“ war bisher ziemlich ausschließlich das Objekt der Untersuchungen. Erst 1906 haben Meyer und Langstein ${ }^{1}$ ) akut schwererkrankte Säuglinge für ihre Acidoseuntersuchungen gewählt. Diese Kinder waren teils völlig bewußtlos, teils leicht benommen und hatten dünne, spritzende Stühle, so daß ich ihre Fälle von "Enterokatarrh" wohl ohne weiteres zu den heute mit „Intoxikation" bezeichneten rechnen darf. Diese Autoren hatten vorher bei gesunden, älteren Kindern ${ }^{2}$ ) und Säuglingèn eine starke Vermehrung der Acetonkörperausscheidung während der Kohlehydratkarenz resp. während des Hungers festgestellt; auch hatten sie beim Säugling eine starke Erhöhung des Ammoniakkoeffizienten während des Hungers nachgewiesen und haben dadurch zum ersten Male bewiesen, daß der Hunger beim Säugling zu einer intermediären Acidose führt. In allen ihren Fällen von „Enterokatarrh" fanden sie ebenfalls einen auffallend hohen Ammoniakkoeffizienten (10-49). Dieser und ein vermehrter Gehalt von flüchtigen Fettsäuren (die viel Alkali binden und diese dadurch dem Körper entziehen) im Stuhl ihrer Kinder, neben der Zuckerausscheidung im Urin, sind nach der Meinung der Autoren beweisend dafür, daß es sich in der Tat bei akut magendarmkranken Kindern um eine Acidose handelt. Ob es sich um eine echte ( $\dot{N}$ a un y $n$ sche Acidosis) oder eine relative Acidose (Steinitz) handelt, ist nicht erwähnt, und weil die Bestimmung der Acetonkörper unterblieben ist, läßt sich die Frage auch nicht entscheiden. Besonders hervorzuheben aber ist, daß Langstein und Meyer in der Acidose nur ein Symptom der Intoxikationserscheinungen, nicht aber die Krankheitsursache selbst erblickten. Zu dieser Ansicht führte sie die Beobachtung, daß therapeutisch ,durch den Hunger, der stets die Acidose vermehren muß, jegliche Intoxikationserscheinung verschwindet".

Durch Langstein und Meyer war der Weg für die weitere Forschung der Acidosefrage im Sinne der Säurevergiftung gewiesen, und man hätte erwarten können, daß sich hier ergebnisreiche Untersuchungen anschließen würden. Es kam aber anders. Die nächstfolgende, durch Finkelstein $^{3}$ ) gewiesene Forschung der Intoxikation nahm eine

1) Meyer u. Langstein, Die Acidose im Kindesalter. II. Mitt.: Die Acidose des Säuglings. Jahrb. f. Kinderheilk. 63, 30. 1906.

a) Langstein u. Meyer, Die Acidose im Kindesalter. I. Mitt.: Die Acidose des älteren Kindes. Jabrb. f. Kinderheilk. 61, 454. 1905.

3) Finkelstein, Über alimentäre Intoxikation. Berlin 1910 (Karger). Sonderabdr. aus Jahrb. f. Kinderheilk. 65-68. 
ganz andere Richtung. Finkelstein, ein scharfsinniger Kliniker, machte in seinen Untersuchungen „Utber alimentäre Intoxikation", die nunmehr klassisch geworden sind, die klinischen Beobachtungen zur Grundlage und zum Ausgangspunkte seiner Arbeit. In klinischen Untersuchungen hatte er in der Nahrung, besonders in dem Zucker, die Intoxikation hervorrufenden Eigenschaften nachgewiesen. Er stellt sich den Mechanismus der Intoxikation in der Weise vor, daß der Darm durch die aus dem Zucker entstehenden giftigen Gärungsprodukte geschädigt wird, und daß auf Grund dieser Schädigung die Molkensalze toxisch wirken. Er ist-der Meinung, daß normalerweise bei der Verdauung und der Resorption von Molkensalzen Veränderungen vor sich gehen, die im Sinne einer Entgiftung derselben wirken, und $\mathrm{da} ß$ sich diese Vorgänge in unzulänglicher Weise abspielen, wenn der Darm erkrankt ist. Daß bei Intoxikation auch saure Produkte des Fett- und Zuckerabbaues zu berücksichtigen sind, leugnet Finkelstein nicht, und bezüglich der Acidose sagt er, da $B$ man noch immer mit der Möglichkeit rechnen muß, ,daß dieselbe nur eins der Symptome darstellt, in denen sich der Zusammenbruch des Organismus offenbart". Auch ist er der Meinung, daß besonders bei der Dekomposition das Fett in dem oben besprochenen Sinne durch Alkaliverlust befördernd auf die relative Acidose wirken kann. Mit diesen Untersuchungen von Finkelstein wurden der alimentäre Einfluß und die Wirkung der Salze in den Vordergrund der Acidosefrage gerückt, und die verschiedensten Arbeiten, die durch seine Untersuchungen angeregt worden sind, lassen ja die ganze Acidose als Faktor bei Intoxikationen im großen und ganzen unberücksichtigt. Ich erwähne von diesen nur Hei $\mathrm{m}^{\mathbf{1}}$ ), der die Intoxikationen in erster Linie als den Ausdruck von Gewebsexsiccation schwersten Grades betrachtet; eine Exsiccation, die durch Salzanhäufung im Blute zu Fiebersteigerungen und anderen typischen Intoxikationssymptomen führt. Auch $J$ undell ${ }^{2}$ ), dem wir die ersten genaueren Kenntnisse über den $\mathrm{N}$ - und Mineralstoffwechsel bei Intoxikationen verdanken, berührt in seiner ausführlichen Arbeit die Acidosefrage gar nicht.

Einen neuen Versuch, die Intoxikation vom Standpunkte der

1) Heim, Die Rolle der Wärmestauung und Exsiccation bei der Intoxikation der Säuglinge. Archiv f. Kinderheilk. 59, 91. 1913.

2) Jundell, Untersuchungen über den Stoffwechsel bei der Dyspepsie und der alimentären Intoxikation. Zeitschr. f. Kinderheilk. 8, 235. 1913. 
Acidose zu betrachten, unternahm Salge $\left.\left.{ }^{1}\right)^{2}\right)$. Er fand bei einem moribunden $\left(1,4 \times 10^{-5}\right)$ und bei einem anderen schwerkranken, einige Tage später verstorbenen toxischen Kinde $\left(6 \times 10^{-7}\right)$ im Blute eine starke Vermehrung der Wasserstoffionenkonzentration; Werte, die sioh den bei künstlich säurevergifteten Tieren gefundenen nähern. Diese Werte stehen vereinzelt da, gegenüber anderen normal gefundenen Werten. Und Salge hat aus ihnen keine weitergehenden Schlüsse bezüglich der Acidosefrage bei Intoxikationen ziehen können. Er betont aber, daß sie als Zeichen von besonderer Labilität bezüglich der regulatorischen Fähigkeiten des Blutes gelten, Labilität, für die noch weiterhin die großen Schwankungen bezüglich der Leitfähigkeit und des osmotischen Druckes im Blute junger Säuglinge bei den verschiedensten Krankheiten mitsprechen, was auch von $\mathrm{Hagner}^{3}$ ) festgestellt und von Bernhard ${ }^{4}$ ) und Rovere ${ }^{5}$ ) in manchen Fällen von Intoxikation auch bei älteren Säuglingen bestätigt wurde.

Inzwischen hatte der Begriff Acidosis durch die biologische und die experimentell-physiologisch-chemische Forschung mancherlei Wandelungen erfahren. Man hatte beim Diabetes, einem sicher acidotischen Zustande, keine Vermehrung der Wasserstoffionenkonzentration im Blute gefunden. Und dies gab zunächst den Anstoß, neue Merkmale für die Acidosis zu suchen. Einige dieser Merkmale werde ich noch näher bei der Beschreibung der Methode in den nächsten Kapiteln besprechen. Ich erwähne hier nur die $\mathrm{CO}_{2}$-Spannung des Blutes. Dieselbe wurde von Porges, Leimdörfer und Markovicib) zuerst in die Klinik eingeführt und dann ziemlich allseitig als ein sicheres Maß

1) Salge, Die Reaktion des Blutserums bei alimentärer Intoxikation des Säuglings. Vorläufige Mitteilung. Zeitschr. f. Kinderheilk, 4, 92. 1912.

2) Salge, Beispiele für die Bedeutung physikalischer und physikalischchemischer Forsebungen in der Physiologie und Pathologie des Säuglings. Zeitschr. f. Kinderheilk. $\gamma, 292.1913$.

3) Hagner, Schwankungen im Eiweißgehalt und in der Leitfähigkeit beim Säuglingsblute. Zeitschr. f. Kinderheilk. 8, 50. 1913.

4) Bernhard, Osmotischer Druck und Eiweißgehalt des Blutes alimentär intoxizierter Säuglinge. Dissert. Leipzig 1913.

5) Rovere, Sulla concentrazione dell'albumina nel siero di sangue nelle gastro-enteriti dei lattanti. Rivista di clinica pediatr. 11, 347. 1913.

B) Porges, Leimdörfer u. Markovici, Über die $\mathrm{CO}_{2}$-Spannung des Blutes in pathologischen Zuständen. Zeitschr. f. klin. Med. 73, 389. 1910. 
für acidotische Zustände anerkannt $\left[\operatorname{Stra~ub}^{1}\right.$ ), Friedericia $\left.{ }^{2}\right)$, La uritzen $\left.\left.{ }^{3}\right)\right]$.

Dieses $\mathrm{MaB}$ hat jedoch durch die Untersuchungen von Hasselbalch $\left.{ }^{4}\right)^{5}$ ) und seine Mitarbeiter eine Beschränkung erfahren. Sie konnten nachweisen, daß neben der Ubbersäuerung des Blutes auch der Reizzustand des Atemzentrums einen großen Einfluß auf den Kohlensäuregehalt des Blutes ausübt.

In der Pädiatrie ist dieses Maß von Howland und Mac Marriott ${ }^{6}$ ) zur Anwendung gekommen. Diese haben 1914 bei toxischen Säuglingen nach der Pleschschen Methode den Kohlensäuregehalt der Alveolarluft bestimmt und dabei gefunden, daß bei Kindern, die Dyspnoe zeigten, der Kohlensäuregehalt tatsächlich vermindert war. Diese, Verminderung wurde durch Zufuhr von Natriumbicarbonat aufgehoben. Diese wichtigen Versuche sprechen nach dem Vorangehenden dafür, daß bei Intoxikationen eine Acidosis vorliegen müsse $\left.\left.{ }^{7}\right)^{8}\right)$.

Recht interessant und völlig abweichend von den oben beschriebenen

1) Straub, Herm., Acidosebestimmungen bei Diabetes mellitus. Klinische Untersuchungen über die Kohlensäurespannung der Alveolarluft. Deutsches Archiv f. klin. Med. 109, 223. 1913.

a) Friedericia, Uber die Bestimmung der diabetischen Acidosis durch Untersuchung der Kohlensäurespannung in der Lungenluft. Zeitschr. f. klin. Med. 80, 1. 1914.

3) Lauritzen, Über Acidosebestimmungen und ihre klinische Anwendbarkeit bei Diabetes mellitus. Zeitschr. f. klin. Med. 80, 13. 1914.

4) Hasselbalch, Neutralitätsregulation und Reizbarkeit des Atemzentrums in ihren Wirkungen auf die Kohlensäurespannung des Blutes. Biochem. Zeitschr. 46, 403. 1912 .

5) Hasselbalch u. Lundsgaard, Blutreaktion und Lungenventilation Skand. Archiv f. Physiol. 27, 13. 1912.

6) Howland u. Mac Marriott, Observations upon the so-called food intoxication of infants with especial reference to the alveolar air. Proc. of the Soc. for exper. Biol. and Med. r, 51. 1914.

7) Dieselben Autoren referierten in New-York Acad. of Med., Sect. on Pediatr. 2. XII. 1915 nach einem Bericht in dem zuletzt zu uns gekommenen Heft Archiv of Ped. 33, 124, Febr. 1916, daß sie bei Intoxikation auch die $\mathrm{O}_{2}$-Dissoziationskurve des Blutes $u$. a. bestimmt und hierbei weitere Anhaltspunkte für die Acidosis gefunden hätten.

8) Anmerk. b. d. Korrektur: Die diesbezügliche Arbeit von Howland und Mac Marriott ist inzwischen in American Journal of Diseases of Children, May 1916. Vol. 11. s. 309-325 ersehienen und erst während der Drucklegung meiner Arbeit zu uns nach Deutschland gekommen. Besonders erwähnenswert ist hier, daß auch diese Autoren schon den Wert der Reaktion des $\mathrm{CO}_{2}$ freien Blutes als acidotischen Indikator erkannt haben. 
Ansichten über Beziehungen zwischen Acidosis und Intoxikation sind die Meinungen der französischen Schule, die ich der Vollständigkeit halber noch zuletzt hinzufügen möchte. Uberall da, wo man diesseits der Säure irgendeine Rolle in der Pathologie der Ernährungsstörungen hat zuschreiben wollen, tritt nach Auffassung der französischen Schule hierfür der Harnstoff auf, der teils als Ursache, teils als Symptom betrachtet wird. Dem Namen Acidosis entspricht hier die Bezeichnung Azotémie. Als ihr prägnantestes Merkmal gilt nach $\mathrm{Hu}_{\text {tinel }}{ }^{1}$ ) u. a. die Vermehrung des Harnstoffes in der Cerebrospinalflüssigkeit. Nobécourt und Marcel Maillet ${ }^{2}$ ) haben bei Atrophie einen vermehrten Harnstoffgehalt (über $0,5 \mathrm{~g}$ pro Liter) der Cerebrospinalflüssigkeit gefunden und betrachten den Gehalt der Cerebrospinalflüssigkeit an Harnstoff als ein $\mathrm{MaB}$ für die Azotémie. Die ,atreptischen“"Zustände sind Folgeerscheinungen von chronischer Azotémie, eine noch hochgradigere Steigerung der Azotémie führt zur Somnolenz: somnolente Form der Azotémie, die gleichzeitig mit unserem Intoxikationszustande zu identifizieren wäre.

\section{Die Methoden.}

\section{A. Die Bestimmung der Acidität oder der wahren Reaktion.}

Eingangs wurde schon kurz angedeutet, daß die Acidität überall in dieser Arbeit durch die Bestimmung der Wasserstoffionenkonzentration festgestellt wurde, und zwar auf elektrometrischem Wege durch die Gaskettenmethode in der Anordnung, wie sie Michaelis ${ }^{3}$ ) zuletzt beschrieben hat. Auf diese Weise konnte die wahre Reaktion angegeben werden, die $\mathrm{ja}$ in biologischer Hinsicht das einzig Wichtige ist; denn von ihr ist der Verlauf der verschiedensten oxydativen und fermentativen Prozesse in hohem Grade abhängig. Die Gaskettenmethode ist die einzige Methode, die einwandfrei gestattet, in einem so komplizierten Gemisch, wie es die Körperflüssigkeiten und -ausscheidungen sind, die Wasserstoffionenkonzentration = die wahre Reaktion zu bestimmen. Die Indikatoren- und Titrationsmethoden versagen hier vollkommen aus verschiedenen Gründen, wie bei Michaelis genauer

1) Hutinel, Les reactions méningées dans l'azotémie chez les nourrissons. Paris médic. 4, 41. 1913.

2) Nobécourt et Marcel Maillet, Chlourémie et azotémie chez les nourrissons. Le Nourrisson 1, 75. 1913.

3) Michaelis, Die Wasserstoffionenkonzentration. Berlin 1914 (Springer). 
besprochen worden ist. Ich erwähne dies nur, weil diese Methoden immer noch zur Bestimmung der Acidität hierbei angewandt werden. Die Gaskettenmethode ist schon einige Male bei pädiatrischen Fragestellungen zur Anwendung gekommen. Ich nenne hier die Namen Pfaundler ${ }^{1}$ ), Allaria ${ }^{2}$ ) und Davidsohn ${ }^{3}$ ). Sie haben in ihren Arbeiten die Methode eingehender besprochen, so daß ich mich auf den Hinweis beschränken kann.

Es wurden überall Doppelbestimmungen, und zwar immer bei Zimmertemperatur, gemacht, die meistens gut miteinander übereinstimmten. In zweifelhaften Fällen wurde noch eine dritte Bestimmung vorgenommen, nur in einzelnen wenigen, wo das Material nicht ausreichte, mußte ich mich leider auf eine Bestimmung beschränken. Die Werte für die Aciditätbestimmungen sind in den am Ende der Arbeit veröffentlichten Protokollen in $P_{H}$, d. h. durch negativen Logarithmus der Wasserstoffionenkonzentration ausgerechnet und eingetragen worden. Diese Art, die Wasserstoffionenkonzentration durch negativen Logarithmus auszudrücken, stammt von Sörensen ${ }^{4}$ ), der dafür der Kürze halber den Namen „Wasserstoffexponent" $=P_{H}$ einführte.

\section{Die wahre Reaktion des Urins.}

Der Urin wurde in sterilen Kölbchen aufgefangen und gleich frisch für die Bestimmung verwendet. Nur im Falle 62 (Kind Köhler) wurde die Acidität im Sammelurin von 24 Stunden bestimmt. Dieser Urin wurde in einem auf Eis stehenden Gefäß gesammelt und mit etwas Toluol versetzt.

2. Die wahre Reaktion des Stuhles.

Der Stuhl wurde in Guttapercha aufgefangen, gleich frisch mit destilliertem, abgekochtem 'Wasser zerrieben, filtriert und das Filtrat gleich für die Bestimmung verwendet.

\section{Die wahre Reaktion des Blutes.}

Die wiederholte Blutgewinnung beim Säugling ist eine ungemein schwierige Prozedur. Früher habe ich das Blut teils durch Inzision aus

1) Pfaundler, loc. cit. S. 277 dieser Arbeit.

2) Allaria, Untersuchungen über Wasserstoffionenkonzentration im Säuglingsmagen. Jahrb. f. Kinderheilk. 6r, 123. 1908. (Erg.-Heft.)

3) Davidsohn, Beitrag zum Chemismus des Säuglingsmagens, Zeitschr. f. Kinderheilk 2, 420. 1911.

4) Sörensen, Enzymstudien II. Biochem. Zeitschr. 21, 131. 1909. 
der Ferse, teils durch Punktion aus der Vena poplitea gewonnen. Diese Wege hatten aber auch ihre Schwierigkeiten, und ich war besonders froh, als ich bei Beginn dieser Arbeit einen Aufsatz von Germain Blechmann ${ }^{1}$ ) in die Hände bekam, der über Blutgewinnung durch Punktion des Sinus longitudinalis durch die große Fontanelle berichtete. Ich habe diese Methode aufgenommen, und zwar mit besonders gutem Erfolg. Nur dank ihr ist es möglich gewesen, systematisch oft wiederholte Blutentnahmen beiSäuglingenzu machen. Die Methode verlangtallerdings eine gewisse Gewandtheit, wenn man sie aber erreicht hat, so ist es eine spielende Leichtigkeit, mit einer $10 \mathrm{ccm}$-Spritze, deren Kolben ich mit Paraffin überziehe, durch Punktion in derhinteren Eckedergroßen Fontanelle aus dem Sinus longitudinalis Blut zu erhalten. Irgendwelche nachteiligen Folgen habe ich nie beobachtet, habe auch bei zahlreichen Sektionen keine intrakranielle Blutung gefunden. In einer später erschienenen Arbeit hebt auch Tobler ${ }^{2}$ ) die Vorzüge der Methode hervor. Das so gewonnene Blut wurde aus der Spritze direkt in kleine $1-2 \mathrm{ccm}$ fassende Elektroden, die bis zu einem Drittel physiologische Kochsalzlösung mit ein paar Körnchen Hirudin enthielten, eingelassen und dann nach der Michaelisschen Schaukelmethode zur Bestimmung der (H') benutzt. Der Rest des Blutes wurde in eine kleine Flasche, die wie oben Kochsalzlösung mit etwas Hirudin, um die Gerinnung zu verhindern, enthielt, ausgespritzt und für weitere Verwendung (Dissoziationskurve resp. Kohlensäureregulationsbreite) bereitgestellt. Bei Leichen wurde das Blut durch Herzpunktion unmittelbar nach dem Tode gewonnen.

4. Die wahre Reaktion der Gewebe einschließlich des Magenund Darminhalts.

Utber die wahre Reaktion der Gewebe des Menschen ist bisher noch nichts bekannt. Auch über die Acidität der Gewebe bei Tieren lagen bis vor kurzem keine Angaben vor. Erst 1914 hat Michaelis $\left.{ }^{3}\right)^{4}$ eine Methode zur Bestimmung der (H') der Gewebssäfte ausgearbeitet. Sie ist sehr einfach und besteht darin, daß man wässrige Extrakte von

1) Blechmann, Technique des prélèvements de sang et des injections intraveineuses chez les nourrissons. Le Nourrisson $2,150.1914$.

2) Tobler, Zur Technik der diagnostischen Blutentnahme und der intravenösen Injektion beim Säugling. Monatsschr. f. Kinderheilk. 13, 384. 1915.

9) Michaelis u. Kramsztyk, Die Wasserstoffionenkonzentration der Gewebssäfte. Biochem. Zeitschr. 62, 180. 1914.

4) Michaelis, Die Bedeutung der Wasserstoffionenkonzentration des Blutes und der Gewebe. Deutsche med. Wochenschr. 40. Jahrg., 1170. 1914. 
zerriebenen Organen ohne weiteres zur Bestimmung benutzt. Weil man auf diese Weise durch spontane Süuerung etwas zu sauere Werte bekommt, hat Michaelis, um diesen Fehler auszuschalten, daneben auch die $\left(\mathrm{H}^{*}\right)$ in wässrigen Extrakten von gleich nach der Entnahme gekochten Organen bestimmt. Diese Werte sind wiederum durch Entweichen der Kohlensäure beim Kochen etwas alkalischer, als die Reaktion im Leben zeigen sollte. Das Mittel von beidenBestimmungen wird nach Michaelis annähernd die Reaktion der Gewebe im Leben anzeigen.

Ich habe mich an die Michaelisschen Vorschriften in der Hauptsache gehalten. Die Leichen wurden sofort nach dem Tode seziert. Ein Teil von allen in Frage kommenden Organen (Muskulatur, Herz, Gehirn, Leber, Milz, Niere) wurde zunächst herausgenommen, zwischen Handtüchern durch Pressen vom Blute befreit und dann in zwei Hälften zerlegt. Die eine wurde sofort in bereitstehendes kochendes Wasser geworfen, die andere Hälfte wurde frisch zerrieben, der Brei mit Wasser versetzt und in dem Filtrat gleich die $\left(\mathrm{H}^{*}\right)$ bestimmt. Nach 5-10 Minuten wurde mit der ersten Hälfte in gleicher Weise verfahren.

Anschließend an die Gewebe wurde auch die Acidität des Inhalts vom Magendarmkanal untersucht. Schon vor Herausnahme der Därme wurden dieselben in situ an bestimmten Stellen abgebunden: 1. am Pylorus, 2. ca. 60-100 cm unterhalb Pylorus, 3. am Ende des Ileums, 4. am Coecum, 5. am Rectum. Danach wurden Magen und Darm herausgenommen, aus dem Inhalt der einzelnen Abschnitte wässrige Extrakte hergestellt und in diesen die $\left(\mathrm{H}^{\prime}\right)$ bestimmt. Nur in einzelnen Fällen wurde der Inhalt mit Wasser verdünnt gekocht und dann erst die $\left(\mathrm{H}^{*}\right)$ bestimmt, um auf diese Weise einen Einblick zu gewinnen, inwieweit die Acidität des Magen- und Darmkanals auf flüchtigen freien Säuren beruht. Das Genauere ist in den Protokollen zu finden.

\section{B. Die Bestimmung der $\mathrm{CO}_{2}$-Regulationsbreite.}

Schon oben wurde angedeutet, $\mathrm{da} B$ Kohlensäure unter gleichbleibendem Reizzustand des Atemzentrums einen Indikator für die Acidosis abgibt. In den diesbezüglichen Untersuchungen hat man versucht, über den Kohlensäuregehalt des Blutes durch Bestimmung des $\mathrm{CO}_{2}$-Partialdruckes in der Alveolarluft Aufsehluß zu bekommen. Diese Bestimmung schien mir aber beim Säugling äußerst schwer durchführbar zu sein ${ }^{1}$ ), und dies führte mich zu folgenden Gedanken: Die $\left(\mathrm{H}^{*}\right)$ des Blutes ist

1) Howland und Marriott ist inzwischen gelungen, durch eine modifizierte Pleschsche Methode auch beim Säugling den $\mathrm{CO}_{2}$-Gehalt der Alveolarluft zu bestimmen, loc. cit. S. 281 dieser Arbeit. 
in hohem Maße abhängig von dem Kohlensäuregehalt des Blutes, wie u. a. Hasselbalch und Lundsgaard') nachgewiesen haben. Wird die Acidität eines und desselben Blutes unter verschiedenen KohlensäurePartialdrucken bestimmt, so ist sie, wie zu erwarten, am höchsten beim höchsten $\mathrm{CO}_{2}$-Gehalt und beim niedrigsten am niedrigsten. Wenn nun ein acidotischer Zustand wie Diabetes vorliegt, so wußte man, daß einerseits die $\left(\mathrm{H}^{*}\right)$ des Blutes unverändert aufrechterhalten wird, während andererseits der $\mathrm{CO}_{2}$-Gehalt in der Alveolarluft zurückgeht. Diese zwei Momente kann man nur dadurch in Einklang bringen, daß die sauren Komponente, die neben $\mathrm{CO}_{2}$ die gesamte Acidität des Blutes ausmachen, vermehrt sein mußten, wie aus dem Schema ohne weiteres ersichtlich ist.

$\left(\mathrm{H}^{*}\right)=$ Konstant $=\mathrm{CO}_{2}+$ übrige saure Komponente.

Da nun in einem gegebenen Falle, wo die (H") des Blutes bei alveolarem $\mathrm{CO}_{2}$-Druck normal, aber die Acidität desselben Blutesnach dem Austreiben von $\mathrm{CO}_{2}$ höher als in anderen Fällen war, so kann man ohne weiteres sagen, daß der $\mathrm{CO}_{2}$-Gehalt im Blute in diesem Falle vermindert sein mußte und an seine Stelle eine Vermehrung der anderen sauren Komponente eingetreten ist. Ich habe nun in mehreren Fällen die wahre Reaktion des Blutes, sowohl die aktuelle als auch die Reaktion des $\mathrm{CO}_{2}$-freien Blutes, untersucht und festgestellt, daß beim gesunden Menschen ziemlich konstante Werte (siehe Genaueres aus den Protokollen) festzulegen sind, Werte, die in einem ziemlich bestimmten Abstand voneinander liegen. Es wurde gefunden:

für $\mathrm{P}_{\text {II }}$ des $\mathrm{CO}_{2}$-freien Blutes normalerweise, abgerundet $\quad=8,45$ für $\mathrm{P}_{\mathrm{H}}$ des frischen, kohlensäurehaltigen Blutes, abgerundet $=7,45$

Differenz durchschnittlich $=\mathbf{1 , 0 0}$

Diese Differenz nenne ich die $\mathrm{CO}_{2}$-Regulationsbreite des Blutes. Beim normalen. Menschen ist sie abgerundet ea. 1,00, was ich von jetzt ab gleich $100 \%$ bezeichne. In der Folge wird analog in jedem Falle aus der Differenz der beiden $\mathrm{P}_{\mathbf{H}}$ durch Multiplikation mit 100 die Prozentzahl für die $\mathrm{CO}_{2}$-Regulationsbreite ermittelt und angegeben.

Ich werde in untenstehendem die verschiedenen Aciditätswerte des Blutes wie folgt bezeichnen: Die aktuelle Reaktion des Blutes, d. h. die Acidität bei alveolarem $\mathrm{CO}_{2}$-Partialdruck, bezeichne ich nach dem Vorschlage von Hasselbalch ${ }^{2}$ ) mit dem Namen regulierte

1) Hasselbalch u. Lundsgaard, loc. cit. S. 281 dieser Arbeit.

2) Hasselbalch, Die „,reduzierte“" und die ,regulierte“" Wasserstoffzahl des Blutes. Biochem. Zeitschr. 74, 56. 1916. 
Wasserstoffachl und die Realtion des $\mathrm{CO}_{2}$-freien Blutes nenre ich „Grundreaktion" resp. "Grund-Wasserstoffzahl". Diese Bezeichnung habe ich gewählt; weil sie am besten darauf hinweist, daß sie Ausdruck für die Grundbestandteile, $d$. $h$. für die nicht flüchtigen Bestandteile des Blutes ist. Die Grund-Wasserstoffzahi bildet gewissermaßen ein Gegenstück zur reduzierten Wasserstoffzahl, welchen Namen Hasselbalch vor kurzem für die Reaktion des Blutes bei $40 \mathrm{~mm} \mathrm{Hg} \mathrm{CO}$-Partialdruck eingeführt hat

Die praktische. Ausführung zur Bestimmung der $\mathrm{CO}_{2}$-Regulationsbreite gestaltete sich folgendermaßen: Zuerst wurde die regulierte Wasserstoffzahl wie oben bestimmt. Dann wurde ein Teil (ca. 2-3 ccm) von demselben Blute in einen Barcroftschen ${ }^{1}$ ) Tonometer gebracht, das ein zylindrisches Gefä $\beta$ von ca. $300 \mathrm{ccm}$ Inhalt ist. Das Gefä $B$ spitzt sich an dem einen Ende zu und ist mit einem Glashahn versehen, an dem anderen Ende befindet sich ein sog. Flaschenhals, der mit einem Gummistöpsel verschlossen werden kann. Das Gefäß wurde dann mittels Wasserstrahlpumpe, die mit dem spitzen Ende des Gefäßes durch Gummischlauch verbunden war, im Wasserbade bei $38^{\circ}$ ca. 5 Minuten lang unter ständigem Umdrehen evakuiert, dann in ein größeres Wasserbad gebracht und bei $38^{\circ} 25$ Minuten lang mittels Motors geschüttelt. Nun wurde das Gefäß geöffnet, das Blut direkt in die Elektrode hineingeleitet und die Grund-Wasserstoffzahl darin bestimmt. Der Abstand zwischen den Werten für die regulierte Wasserstoffzahl und für die Grund-Wasserstoffzahl ist gleichbedeutend mit der $\mathrm{CO}_{2}$-Regulationsbreite, die nach Multiplikation mit 100 in \% ausgedrückt wird.

\section{Kritik der Methode.}

Diese meine Methode scheint mir in besonders einfacher Weise einen guten Einblick in die Regulationsfähigkeit des Blutes zu gestatten, ohne daß man mit den umständlichen $\mathrm{CO}_{2}$-Partialdrucken zu manipulieren hat. Sie gibt nach zweimaliger Bestimmung der $P_{\mathbf{H}}$ bei ein und demselben Blute ohne weiteres Aufschluß über die Kohlensäuremenge des Blutes und über das Verhalten, d. h. Verminderung resp. Vermehrung der anderen sauren Bestandteile in ihm, also gerade über die Fragen, die uns bezüglich der Zusammensetzung des Blutes in acidotischen Zuständen besonders interessieren.

Aber auf zwei Punkte muß man bei der Methode achten, die Länge

1) Bareroft, The respiratory function of the blood. Cambridge 1914 (Monographie).

Zeltschrift für Kinderheilkunde. Orig. XIV. 
der Schüttelung und die damit verbundene Selbstsäuerung. Es ist wichtig, daß man im Wasserbade genügend lange schüttelt. Meistens genügen 15 Minuten, ich habe vorsichtshalber regelmäßig 25 Minuten lang geschüttelt. Längeres Schütteln bringt aber leicht Selbstsäuerung des Blutes mit sich. Die Selbstsäuerung tritt nicht in nennenswertem Maße bei im Leben entnommenem Blute auf, aber oft im Blute, das nach dem Tode entnommen wird: Auch möglichst sauberes Arbeiten ist Vorbedingung. Ich habe vorsichtshalber meine Glassachen, Tonometer usw. meistens erst nach Sterilisation benutzt.

Bei Fällen, in denen eine Acidosis mit saurem Blute, d. h. mit einem $\left(\mathrm{H}^{*}\right)$ größer als normalerweise vorliegt, kann die $\mathrm{CO}_{2}$-Regulationsgrenze unter Umständen annähernd normal gefunden werden. Hier sind beide Werte, sowohl die Grund-Wasserstoffzahl wie die regulierte Wasserstoffzahl etwa in gleichem Verhältnis größer geworden. Die $\mathrm{CO}_{2}$-Regulationsbreite allein könnte hier keinen Aufschluß über die tatsächlich vorhandene Acidität geben. Aber dies ist auch nicht notwendig, denn die vergrößerte regulierte Wasserstoffzahl sagt schon allein, daß da eine Acidosis vorliegt und die $\mathrm{CO}_{2}$-Regulationsbreite ist ja nur dazu da, um die verschleierten acidotischen Zustände, in denen der Körper noch imstande ist eine normale regulierte Wasserstoffzahl aufreohtzuerhalten, zu erkennen. In diesen letzterwähnten Fällen ist die $\mathrm{CO}_{2}$-Regulationsbreite ein zuverlässiger Indicator für den acidotischen Zustand.

Als Beispiel für die Leistungsfähigkeit der Methode entnehme ich den Protokollen folgende Versuche:

Blut vom gesunden Erwachsenen (mit Spritze aus der Armvene entnommen).

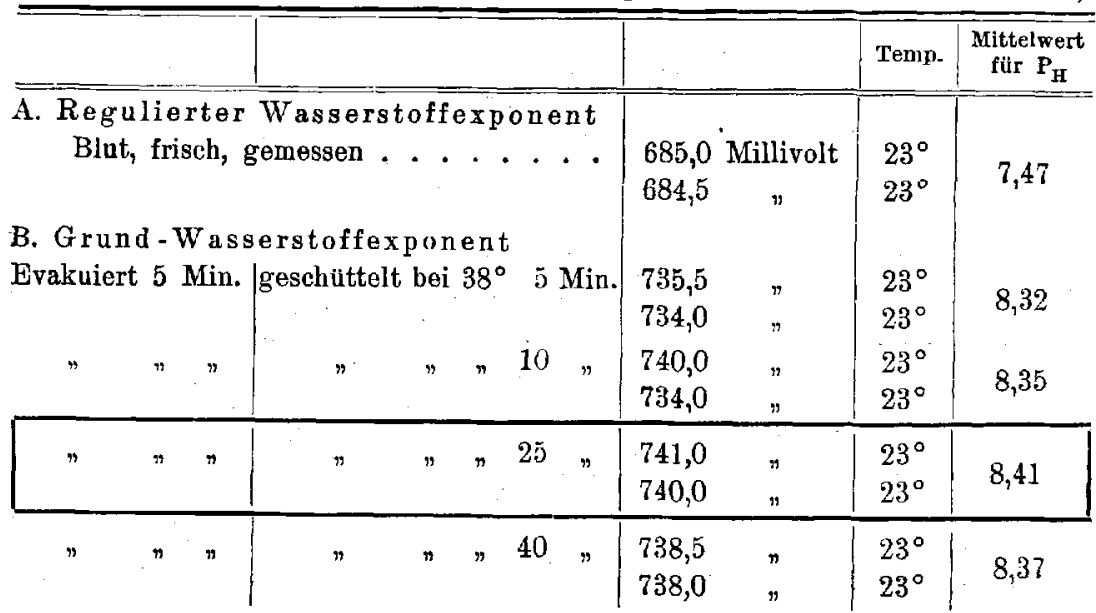

$\mathrm{CO}_{2}$-Regulationsbreite von $8,41-7,47=0,94=94 \%$. 
und Intoxikationsacidosis in ihren Beziehungen zueinander.

Blut von einem schwer toxischon Kinde (einen Tag vor dem Tode durch Sinuspunktion entnommen).

\begin{tabular}{|c|c|c|c|c|}
\hline & & & Temp. & $\begin{array}{c}\text { Mittelwert } \\
\text { fü } \mathbf{P}_{\mathbf{H}} \\
\end{array}$ \\
\hline $\begin{array}{l}\text { A. Regulierter } \\
\text { Blut, frisch, }\end{array}$ & $\begin{array}{l}\text { Wasserstoffexponent } \\
\text { gemessen ....... }\end{array}$ & $\begin{array}{l}683,0 \text { Millivolt } \\
688,0 \quad\end{array}$ & $\begin{array}{l}18^{\circ} \\
18^{\circ}\end{array}$ & 7,50 \\
\hline $\begin{array}{l}\text { B. Grund-Wass } \\
\text { Erakuiert } 5 \text { Min. }\end{array}$ & $\begin{array}{l}\text { serstoffexponent } \\
\text { geschüttelt bei } 38^{\circ} 15 \mathrm{Min} \text {. }\end{array}$ & $\begin{array}{l}695,0 \\
697,0\end{array}$ & $\begin{array}{l}18^{\circ} \\
18^{\circ}\end{array}$ & 7,72 \\
\hline$n$ & $" \quad " 30$, & $\begin{array}{l}696,0 \\
697,0\end{array}$ & $\begin{array}{l}18^{\circ} \\
18^{\circ}\end{array}$ & 7,73 \\
\hline
\end{tabular}

$\mathrm{CO}_{2}$-Regulationsbreite von $7,73-7,50=0,23=23 \%$.

Herzblut von einem an Intoxikation verstorbenen Kinde.

\begin{tabular}{|c|c|c|c|c|}
\hline & & & Temp. & $\begin{array}{c}\text { Mittelwert } \\
\text { für } \mathbf{P}_{\mathbf{H}}\end{array}$ \\
\hline $\begin{array}{l}\text { A. Regalierter } \\
\text { Blut, frisch, }\end{array}$ & $\begin{array}{l}\text { Wasserstoffexponent } \\
\text { gemessen } \cdots \therefore \therefore .\end{array}$ & $\begin{array}{l}642,0 \text { Millivolt } \\
641,0 \quad\end{array}$ & $\begin{array}{l}17^{\circ} \\
17^{\circ}\end{array}$ & 6,79 \\
\hline $\begin{array}{l}\text { B. Grund-Wass } \\
\text { Evakuiert } 5 \mathrm{Min} \text {. }\end{array}$ & $\begin{array}{l}\text { erstoffexponent } \\
\text { geschuttelt bei } 38^{\circ} 15 \mathrm{Min} .\end{array}$ & $\begin{array}{l}665,0 \\
664,0 \quad n\end{array}$ & $\begin{array}{l}17^{\circ} \\
17^{\circ}\end{array}$ & 7,20 \\
\hline$\ddot{n}$ & $" \quad n 25 n$ & $\begin{array}{l}665,0 \\
667,0\end{array}$ & $\begin{array}{l}17^{\circ} \\
17^{\circ}\end{array}$ & 7,22 \\
\hline$n \quad n \quad n$ & $n \quad n \quad n \quad 35 \ldots$ & $\begin{array}{ll}653,0 & n \\
657,0 & n\end{array}$ & $\begin{array}{l}17^{\circ} \\
17^{\circ}\end{array}$ & 7,03 \\
\hline
\end{tabular}

$\mathrm{CO}_{2}$-Regulationsbreite von $6,79-7,22=0,43=43 \%$.

Die Beispiele zeigen, daß 5 Minuten langes Evakuieren und $25 \mathrm{Mi}-$ nuten langes Schütteln vollkommen genügen, um $\mathrm{CO}_{2}$ so entweichen zu lassen, daß eine konstante Reaktion im Blute hervorgerufen wird ${ }^{\mathbf{1}}$ ). In Fällen, in denen keine Selbstsäuerung eingetreten ist, sind die Fehlerquellen der Methode gleich denen der Gaskettenmethode überhaupt, die ja, wie bekannt, eine der exaktesten biologischen Methoden ist.

1) Ob das Blut jetzt vollkommen $\mathrm{CO}_{2}$-frei ist, kann ich nicht ohne weiteres sagen. Die Konstanz der ( $\left.\mathrm{H}^{*}\right)$ zeigt aber, daß die Dissoziation der $\mathrm{CO}_{2}$ aus dem Natriumbicarbonat und aus anderen lookeren $\mathrm{CO}_{2}$-haltigen Verbindungen zu Ende geführt worden ist. 


\section{Die Dissoziationskurve des Oxyhämoglobins im Blute.}

Barcroft ${ }^{1}$ ) hat durch jahrelange mühevolle Arbeit mit seinen Mitarbeitern die Gesetze der $\mathrm{O}_{2}$-Bindung des Hämoglobins geschaffen. Er hat die Früchte seiner Arbeiten zur Nutzanwendung auch auf die menschliche Pathologie und Physiologie übertragen, indem er u. a. die Beziehungen der $\mathrm{O}_{2}$-Dissoziationskurve $=\mathrm{O}_{2}$-Bindungskurve des menschlichen Blutes zu den verschiedenen pathologischen und physiologischen Zuständen klarlegte. Uns interessiert an dieser Stelle besonders sein Befund, daß die Dissoziationskurve des Blutes bei Urämie und Diabetes einen abnorm niedrigen Verlauf nimmt, etwa wie bei Fällen, in denen man Süure künstlich in das Blut geführt hatte. Er betrachtet demnach eine $\mathrm{O}_{2}$-Dissoziationskurve, die einen niedrigeren Verlauf hat als in der Norm, als Ausdruck eines acidotischen Zustandes. Unter Acidose versteht er einen Zustand, in welchem abnorme Mengen von nicht flüchtigen Säuren im Körper kreisen, die eine Verdrängung der $\mathrm{CO}_{2}$ bewirken. Die Dissoziationskurve des Blutes ist demnach ein empfindlicher Indicator etwaiger Acidose des Blutes. Von diesem Gedanken ausgehend, habe ich auch bei Säuglingen Untersuchungen über den Verlauf der Dissoziationskurve bei den verschiedensten Zuständen vorgenommen.

Zuerst muß ich wohl kurz angeben, was man mit der Dissoziationskurve des Blutes meint. Die Dissoziationskurve stellt eine leicht Sförmige Linie dar, welche die prozentuelle Sättigung des Blutes mit Sauerstoff bei Änderung des $\mathrm{O}_{2}$-Partialdruckes anzeigt. Sie wird dadurch ermittelt, daß man das Blut mit Gasmischungen von bekanntem $\mathrm{O}_{2}$-Gehalt in Berührung bringt und nach dem Eintreten des Gleichgewichtes bestimmt, zu wieviel Prozent der maximalen Sättigung das Blut bei dem bekannten $\mathrm{O}_{2}$-Partialdruck der Gasmischung mit $\mathrm{O}_{2}$ gesättigt ist. Der $\mathrm{O}_{2}$-Partialdruck wird als Abszisse, die zugehörige prozentuelle Sättigung als Ordinate aufgetragen. Bestimmt man dann die prozentuelle Sättigung bei verschiedenen $\mathrm{O}_{2}$-Partialdrucken, so bekommt man auf dem Koordinatensystem mehrere Punkte; vereinigt man diese, so hat man eine Linie, die man $\mathrm{O}_{2}$-Dissoziationskurve des Blutes nennt. Bei der Bestimmung der Dissoziationskurve habe ich mich im großen und ganzen an die Vorschriften von Barcroft gehalten. Alle meine Dissoziationskurven weichen aber in einer Hinsicht prinzipiell von den Barcroftschen ab. Sie gelten alle für

1) Barcroft, loc. cit., S. 287 dieser Arbeit. 
Blut, das wie oben beschrieben $\mathrm{CO}_{2}$-frei gemacht wurde, im Gegensatz zu den Barcroftschen Kurven, die bei einem $\mathrm{CO}_{2}$ Partialdruck von $40 \mathrm{~mm} \mathrm{Hg}$ bestimmt worden sind. Schon durch $\mathrm{Bohr} \mathrm{r}^{1}$ ) wußte man, daß die $\mathrm{CO}_{2}$ erniedrigend auf den Verlauf der Dissoziationskurve wirkt; meine Kurven nehmen daher einen höheren Verlauf, als wenn sie bei $40 \mathrm{~mm} \mathrm{Hg}$ bestimmt worden wären.

Zur genaueren Orientierung über die Methode verweise ich auf die Monographie von Barcroft ${ }^{2}$ ), hier muß ich aber folgende Winzelheiten über die Ausführung der Bestimmungen herausgreifen:

Das zur Bestimmung zu benutzende Blut (ca. $2 \mathrm{ccm}$ ) wurde frisch in das Barcroftsche Tonometer (siehe oben) gebracht, dann wie oben von $\mathrm{CO}_{2}$ befreit und nachher mit Gasgemisch gefüllt, das nur aus $\mathbf{N}$ und $\mathrm{O}_{2}$ bestand, in den erforderlichen und vorher bestimmten Verhältnissen. Danach wurde das Tonometer in ein großes Wasserbad gebracht und darin mittels Motors bei $38^{\circ} 25$ Minuten lang geschüttelt; der Uberdruck wurde nach ein paar Minuten durch Offnen des Tonometerhahns ausgeglichen. Dann wurde das Tonometer aus dem Wasserbade herausgenommen und durch das spitze Ende ca. 0,1 ccm Blut, ohne in Berührung mit der Luft zu kommen, unter Ammoniak in die kleine Birne eines Barcroftschen Differenzialmanometers ${ }^{3}$ ) (für $0,1 \mathrm{ccm}$ Blut) geleitet. Das Differentialmanometer wurde dann in ein Wasserbad, meistens von Zimmertemperatur, gebracht und gewartet, bis das Gleichgewicht der Nelkenölmenisken eingetreten war. Dann wurde vorsichtig geschüttelt, wobei das zu untersuchende ungesättigte Blut $\mathrm{O}_{2}$ aus der kleinen Birne aufnahm. Dabei stieg der Meniscus in dem gleichseitigen Schenkel des Manometers. Die Abstandsdifferenz der Menisken wurde notiert, dann wurden die Hähne meistens geöffnet. Nach Verschließen der Hähne und nach eingetretenem Gleichgewicht wurde durch Neigen des Manometers ein Tropfen Kaliumferricyanid, der in der Ausstülpung der Birne lag, in das Blut geleitet und das Manometer weitergeschüttelt. Nach eingetretener Konstanz der Meniskenlage wurde deren Abstandsdifferenz notiert. Aus diesen zwei Differenzen habe ich dann die prozentuelle Sättigung in folgender einfacher Weise ohne weitere Korrektionen berechnet.

1) Bohr, Handbuch der physiologischen Methodik (Tigerstedt), II. Bd., 1. Hälfte, S. $1 \mathrm{ff}$.

2) Barcroft, loc. cit. S. 287; daselbst S. 296. Siehe auch Franz Müller, Die Eigenschaften des roten Blutfarbstoffes. Handb. d. Biochem., Erg.-Bd. 1913, S. 118.

3) bei Bleckmann \& Burger, Berlin N, Auguststr. 3 a nach meinen speziellen Angaben hergestellt. 
Bezeichnen wir die $\mathrm{O}_{2}$-Menge, die in dem zu untersuchenden Blute vor dem Schütteln vorhanden war, mit $X$; die Abstandsdifferenz nach dem ersten Schütteln war z. B. = 1 und nach der Zufuhr von Kaliumferricyanid, das ja bekanntlich $\mathrm{O}_{2}$ quantitativ aus dem Blute austreibt, $=4$, so ist

$$
4=1+\mathrm{X} \text { und daher } \mathrm{X}=3 \text {, }
$$

d. h. das. Blut war. zu $3 / 4$ gesättigt $=75 \% \quad \mathrm{O}_{2}$-Sättigung. Nachdem die $\mathrm{O}_{2}$-Sättigung bestimmt worden war, wurden aus demselben Tonometer je ca. $25 \mathrm{ccm}$ Gas zur Analyse zweimal entnommen. Die Gasanalysen wurden im Haldaneschen ${ }^{1}$ ) Gasanalysenapparat bestimmt. Hatte man nun den $\mathrm{O}_{2}$-Gehalt des Gasgemisches aus dem Tonometer ermittelt, so konnte man mit Hilfe des jeweiligen Barometerdruckes den $\mathrm{O}_{2}$-Partialdruck in $\mathrm{mm} \mathrm{Hg}$ im Tonometer angeben. Aus diesen zwei Zahlen: prozentuelle Sättigung und $\mathrm{O}_{2}$-Partialdruck in $\mathrm{mm} \mathrm{Hg}$ konnte ein Punkt für die Dissoziationskurve des untersuchten Blutes festgestellt werden, wie oben angegeben worden ist.

Ich hatte zwei Differenzialmanometer zur Verfügung und konnte daher denselben Punkt zweifach bestimmen. Die Differenz zwischen beiden Bestimmungen lag meistens $\pm 5 \%$, wie aus den Protokollen ersichtlich.

Zur Bestimmung eines weiteren Punktes an anderer Stelle der Dissoziationskurve habe ich immer frisches, bis dahin auf Eis gestandenes Blut in das Tonometer eingeführt, weil die Manipulationen so lange dauerten, daß Gefahr der Selbstsäuerung vorlag, wenn ich dasselbe Blut dem ganzen Verfahren nochmals hätte unterwerfen müssen.

Bisweilen habe ich noch einen dritten Punkt auf der Dissoziationskurve bestimmt, meistens sie aber nach der Hillschen ${ }^{2}$ ) Formel

$$
\frac{\mathrm{y}}{100}=\frac{\mathrm{Kx}^{\mathbf{n}}}{1+\mathrm{K}^{\mathbf{n}}}
$$

rechnerisch ermittelt. In dieser Formel bedeutet $\mathrm{y}=$ die prozentuelle $\mathrm{O}_{2}$-Sättigung, $\mathrm{x}=\mathrm{O}_{2}$-Partialdruck in $\mathrm{mm} \mathrm{Hg}, \mathbf{n}=$ Anzahl der aggregierten Hämoglobinmoleküle. Praktisch ist im normalen Blute $n=2,5$. Ich habe in pathologischen Fällen $n=1,5-2,5$ gefunden. Das meist Variable ist $\mathrm{K}$. Beim acidotischen Zustande ist es am kleinsten. Mit Hilfe dieser Formel kann die ganze Dissoziationskurve gebaut werden, wenn man einmal zwei Punkte bestimmt hat. Sie gestattet ebenfalls eine gute Kontrolle der experimentell gefundenen Punkte.

1) ebenfalls bei Bleckmann \& Burger erhältlich.

2) Siehe Barcroft, loc. cit. S. 287. 


\section{Untersuchungen an jungen Säuglingen (Frühgeborenen und Neugeborenen).}

A. Die wahre Reaktion des Urins und des Stuhls bei steigendem Alter und bei verschiedener Ernährung.

Es heißt bisher, daß nur dank fortdauernder, ununterbrochener Ausscheidung der sauren Stoffwechselprodukte das Blut imstande ist, eine so konstante Reaktion aufrechtzuerhalten, wie man sie gefunden hat. Diese allein herrschende Rolle der Nieren bei der Ausscheidung der sauren Nahrungs- und Körperprodukte ist schon zu einem so feststehenden Lehrsatz geworden, daß niemand an andere Ausscheidungsmöglichkeiten gedacht hat. Von diesem Gedanken ausgehend habe auch ich beim Beginn meiner Untersuchungen über den Wasserstoffionenstoff wechsel im Säuglingsorganismus neben dem Blute nur den Urin berücksichtigt. Später wurde ich eines Besseren belehrt, indem ich auch in dem Darm einen wichtigen Faktor bei der Regulation des Wasserstoffionenstoff wechsels im Säuglingsorganismus erblickte. Es stellte sich bald heraus, daß Darm und Nieren bei Säuglingen in sehr nahen Wechselbeziehungen bezüglich der Ausscheidung der sauren Produkte stehen.

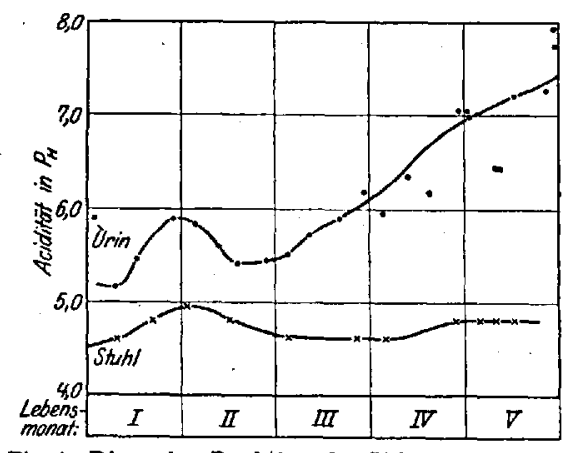

Fig. 1. Die wahre Reaktion des Urins und Stuhles in den ersten Lebensmonaten bei einer Frühgeburt. Fall 62. Geburtsgewicht $960 \mathrm{~g}$.

1. Urin allein (Protokolle Nr. $1-26$ und 60-63).

Zunächst untersuchte ich bei einer Reihe von kleinen Frühgeburten von $1000-1500 \mathrm{~g}$ Geburtsgewicht in verschiedenem Alter die Reaktion des Urins in Einzelproben und konnte dabei feststellen, daß die älteren und kräftigeren Frühgeburten in der Regel bei Frauenmilchernährung einen weniger sauren Urin als die jüngeren ausschieden. Bei systematischen, teils täglichen, teils wöchentlich einmal wiederholten Untersuchungsreihen konnte ich' dann festatellen, da $B$ in der Tat die Urinacidität bei einer und derselben Frühgeburt Neigung hat, allmählich geringer zu werden. Ein gutes Beispiel gibt Fall $1 \mathrm{ab}$, wo die ersten Werte am Anfang der Untersuchungsreihe im Alter von 24 Tagen um $\mathbf{P}_{\mathbf{H}}=$ ca. 5,5 schwanken, und wo die letzten Werte im Alter von $31 / 4-33 / 4$ Monaten um $P_{H}=$ ca. 7,2 liegen. 
Den schönsten diesbezüglichen Versuch zeigt aber der Fall 62. Bei diesem Kinde, das mit einem Gewicht von $960 \mathrm{~g}$ geboren wurde, konnte im Sammelurin von 24 Stunden bei gleichbleibender Nahrung (Frauenmilch) eine ununterbrochene Untersuchungsreihe von 6 Monaten vorgenommen werden. Die folgende Durchschnittstabelle zeigt die Zahlen:

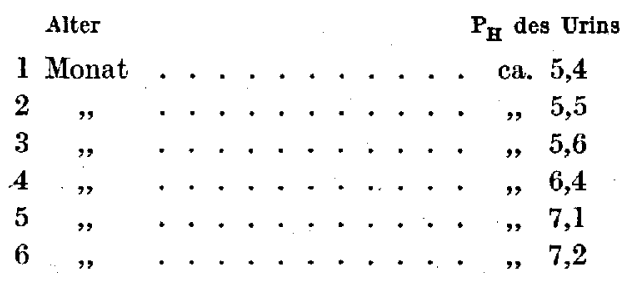

die außerdem auf der Fig. 1 graphisch dargestellt sind. Da sehen wir, wie die Acidität eine ziemlich gleichmäßige Neigung zeigt, mit dem zunehmenden Alter kleiner zu werden, d. h. $\mathbf{P}_{\mathbf{H}}$ wird größer.

Im Fall 3 sehen wir in etwas kürzerer Periode dieselbe Steigerung der $\mathbf{P}_{\mathbf{H}}$-Zahlen; im Fall $\mathbf{7}$ ebenfalls. Bei den anderen Frühgeburten hatte ich keine Gelegenheit, so langdauernde systematische Untersuchungen bei Frauenmilchernährung anzustellen, weil wegen Frauenmilchmangels eine größere Zahl von Frühgeburten längere Zeit nicht ausschließlich mit Frauenmilch ernährt werden konnte. Aber auch in kürzeren Beobachtungen kommt diese Neigung bereits mehr oder minder klar zutage. Eine Ausnahme bildet der Fall 24, wo der Urin schon in den ersten Tagen die Acidität von $P_{\mathbf{H}}$ ca. 7,0 zeigte. Auch Fall 25 hatte ebenfalls in den ersten Tagen $P_{\text {H }}$ ca. 6,70, es handelt sich aber dabei um ein pathologisches Kind mit kongenitalem Herzfehler und Durchfall, so daß ich diesen Fall nicht mit den anderen vergleichen darf. Bezüglich der Frühgeburten muß ich schon an dieser Stelle erwähnen, daß der Urin im allgemeinen in den ersten Zeiten merkbar saurer war als z. B. bei normalen Neugeborenen, bei denen ich ebenfalls Serienuntersuchungen angestellt habe.

Da lagen die Verhältnisse im Prinzip ähnlich wie bei den Frühgeburten. Die Acidität des Urins in den ersten Tagen war meistens auch auffallend hoch. $P_{\mathbf{H}}$ schwankte von ca. 5,4-5,9, aber im Gegensatz zu den Frühgeburten fing sie schon meist in der zweiten Woche an kleiner zu werden, so daß $P_{\mathbf{H}} \mathbf{6 , 2 - 7 , 0} \mathrm{zu}$ finden war. Als typisches Beispiel von diesen Verhältnissen mögen nachstehende Zahlen dienen: 
Fall 17. Neugeborenes, gesund (Geb.-Gew. $2700 \mathrm{~g}$ ).

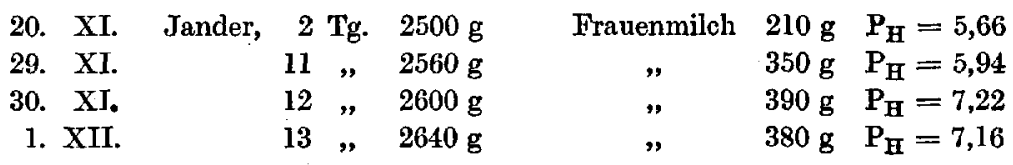

Die Zahlen für die Urinacidität bei den Neugeborenen schwanken übrigens in viel größeren Grenzen als bei den Frühgeburten, wodurch die Ubersicht hier weniger gut zur Geltung kommt. Dies mag wohl teilweise damit zusammenhängen, daß die Frühgeburten fast regelmäBig dreistündlich, auch in der Nacht, ihre Nahrung bekamen, während die Neugeborenen nur am Tage angelegt wurden. Bei Frühgeburten kann also unter keinen Umständen Hungerwirkung als ein die Urinacidität erhöhender Faktor in Frage kommen. Sie nahmen auch sämtlich während der Versuche regelmäßig zu. Bei Neugeborenen könnte .man dagegen in den ersten 3-4 Lebenstagen von einem gewissen Hungerzustand sprechen, dann aber fingen sie an züzunehmen und zeigten trotzdem noch eine Zeitlang auffallend hohe Acidität (Fall 16), eine Acidität, die dann im späteren Alter bei einem mit Frauenmilch ernährten, gesunden Säugling nicht mehr zu treffen war.

Wie sind nun diese hohen Aciditätswerte bei Neugeborenen in den ersten Lebenstagen und bei Frühgeburten in den ersten Lebensmonaten zu erklären? Der erste Gedanke ist natürlich, daß sie als Ausdruck einer Ubersäuerung des Organismus aufzufassen sind. Man könnte sich ja vorstellen, daß bei diesen Kindern aus irgendeinem uns vorläufig unbekannten Grunde in dem intermediären Stoffwechsel mehr saure Produkte als im späteren Alter auftreten. So plausibel diese Annahme auch im ersten Augenblick erscheint und so oft man auch den Gedanken trifft, daß die Urinacidität ein Ausdruck von im Uberfluß ausgeschiedenen sauren Produkten sei, so ist sie doch nicht ohne weiteres zulässig. Denn in solchem Gemisch, wie der Harn, gibt ja die Acidität gar keinen Aufschluß darüber, wieviel Säure im neutralisierten Zustande, die ja auch dem Blute entrissen worden ist, der Harn enthält. Wenn auch die Acidität keinen Aufschluß über die absolute Menge der ausgeschiedenen sauren Produkte gibt, so gibt sie jedenfalls, was uns wichtig ist, einen sicheren Beweis dafür, daß der Körper die sauren Stoffwechselprodukte, relativ betrachtet, weniger neutralisiert zur Ausscheidung bringt. Eine andere Frage ist dann, ob das mit irgendeinem Mangel an neutralisierendem Alkali, Ammoniak usw. zusammenhängt, oder ob es eine besondere Eigentümlichkeit dieses Organismus ist, 
die sauren Produkte zu geringerem Teil neutralisiert als normal zur Ausscheidung zu bringen. Um diese wichtige kardinale Frage zu klären, genügten natürlich nicht meine wenigen Urinuntersuchungen. Und andere Urinuntersuchungen bezüglich der wahren Reaktion bei Neugeborenen und Frühgeburten gab es nicht, ebensowenig wie überhaupt bei Säuglingen. Die einzigen bisherigen Versuche, die sich die Ermittlung der Acidität des Urins bei Säuglingen zum Thema gestellt haben, stammen von Keller') und Freund ${ }^{2}$ ), beziehen sich auf ,magendarmkranke" Säuglinge und sind mit der Titrationsmethode gemacht, die ja wie schon erwähnt, keinen Aufschluß über die wahre Reaktion des Urins geben kann. Klarheit hierüber könnte nur durch weitere, breiter angelegte Versuche geschaffen werden. Jedenfalls lag kein Grund vor, bei diesen Neugeborenen und besonders bei Frühgeburten einen chronischen Alkalimangel vorauszusetzen. Die Frühgeburten hatten während der Versuchsperioden ganz normale Brustmilchstühle und litten nicht unter Darmkatarrhen, die einen Alkaliverlust hätten verursachen können. Von vornherein war mir demnach mehr wahrscheinlich, daß die erhöhte Acidität des Urins hier in Zusammenhang mit etwaiger besonderer Eigentümlichkeit des Frühgeburten- bzw. Neugeborenenorganismus stand. Ich meinerseits habe mich bemüht, durch weitere Versuche dieser Frage näher zu treten, die ich jetzt folgen lasse:

\section{Urin und Stuhl bei Frauenmilch- und bei Kuhmilch- ernährung.}

Bei der Untersuchung der Frühgeburtenurine war es mir schon aufgefallen, daß Veränderungen der Nahrung, z. B. der Utbergang von Frauenmilch zur Kuhmilch, einen starken Einfluß auf die Reaktion des Urins ausübten. Um die Verhältnisse klarzulegen, habe ich besondere Untersuchungen vorgenommen, in welchen ich das Verhalten des Urins und des Stuhls bei Veränderung der Nahrung studieren wollte. Es zeigte sich dabei, daß hier bezüglich der Acidität ganz gesetzmäßige Verhältnisse vorliegen. Bezüglich der Acidität des Stuhles möchte ich zuerst erwähnen, daß sich bei Frauenmilchernährung eine auffallend starke Acidität zeigte, die monatelang nur unerwartet geringen Schwankungen unterworfen war. Dies im Gegensatze zu den Aciditätswerten

1) Keller, Über die Bedeutung der Acidität des Harns beim magendarmkranken Säugling. Jahrb. f. Kinderheilk. 47, 176. 1898.

2) Freund, Säuren und Basen im Urin kranker Säuglinge. Monatsschr. f. Kinderheilk. 1, 230. 1902 . 
des Urins, die großen Schwankungen und außerdem Verschiebungen mit zunehmendem Alter unterworfen waren. So z. B. bewegten sich in dem oben besprochenen Fall 62, wo ich imstande war, bei Frauenmilchernährung, neben dem Urin auch den Stuhl 6 Monate lang systematisch zu untersuchen, die Schwankungen in der Stuhlacidität zwischen $P_{\mathrm{H}}$ ca. $4,60-5,20$, dagegen in der Urinaciditït zwischen $P_{\mathbf{H}}$ 5,18-7,15; Verhältnisse, die ich auf Fig. 1 graphisch dargestellt habe.

Bei demselben Kinde wurde im Alter von 6 Monaten Nahrungsveränderung vorgenommen: Ưbergang von Frauenmilch auf $1 / 2-$ Milch (2\% Larosan, 5\% Rohrzucker). Dabei zeigte sich folgendes: die Reaktion des Stuhles, die bisher konstant stark sauer gewesen war (siehe Protokolle S. 407), schoß gleich vom folgenden Tage an hinauf, hoch ins alkalische Gebiet $\left(P_{\mathbf{H}}\right.$ von ca. 5,0 bis ca. 8,0 ), die bisher leicht alkalische Reaktion des Urins sank dagegen ins saure Gebiet $\left(P_{\mathrm{H}}\right.$ ca. $\left.7,60-6,20\right)$. Die Verhältnisse veranschaulicht. Fig. 2, wo das eigentümliche Kreuzen der Urin- und Stuhllinien besonders augenfällig zu Tage tritt.

Dieses Verhalten beruhte auf keinem Zufall, es wiederholte sich bei jeder gleichen .Untersuchung, so bei Fall 26, wo mitten in der Frauenmilchernährung eine 10 -

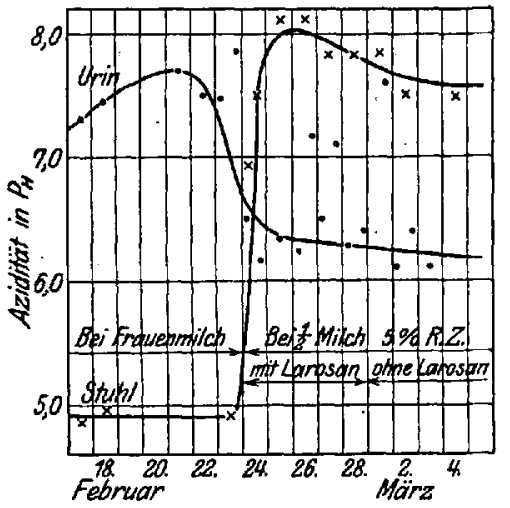

Fig. 2. Die Aclditit des Stuhls und Urins bel Ubergang von Frauenmilch auf $1 / 2-$ Milch. Fall 62: Fruhgeburt Kö h l er, Alter ca.6 $6 \mathrm{Mon}_{\text {, }}$ Gewicht $2650 \mathrm{~g}$.

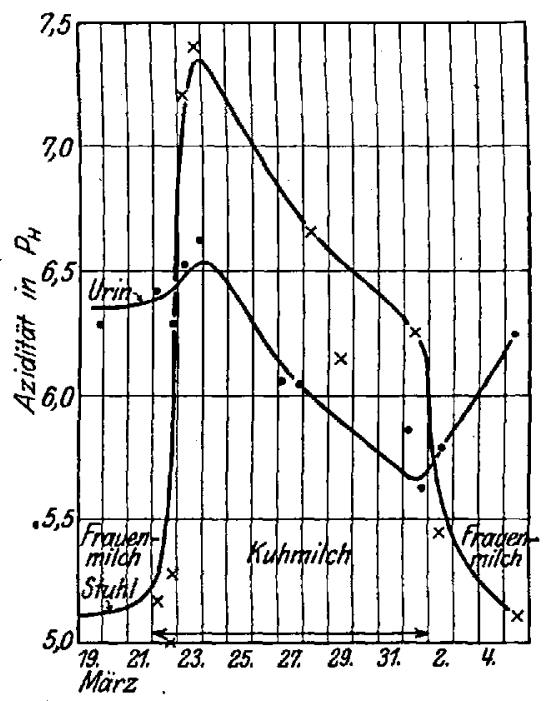

Fjg. 8. Die Acldităt des Stuhls und Urins bei Frauenmilch-und Kuhmilchernlihrung. Fall 26: Frühgeburt $G$ rieb ner, 31/2 Mon., Gewicht 3200 g. tägige Kuhmilchperiode eingeschoben wurde (siehe Protokoll S. 380) ${ }^{1}$ ).

1) Dr. Eitel, der bei uns die wahre Reaktion der Stühle bei verschiedener Ernährung in einer besonderen Arbeit untersucht hat, hat auch ständig dasselbe 
Hier stieg die Acidität des Stuhls ebenfalls zunächst von $P_{H}$ ca. 5,0 bis 7,80 und sank erst nach nochmaligem Utbergang zur Frauenmilchernährung zu den alten Werten bei $P_{\mathrm{II}}$ ca. 5,0. Die Urinreaktion sank dagegen etwas langsamer von $P_{H} 6,40-5,70$, um später wieder bis $P_{H}$ ca. 6,30 anzusteigen. Der Versuch ist auf Fig. 4 graphisch dargestellt.

Bezüglich des Urins hatte ich schon dieselbe Neigung, sauer zu werden, bei Utbergang von Frauenmilch zu Kuhmilchmischungen konstatiert. Weil aber bei jüngeren Frühgeburten, die mit Frauenmilch ernährt werden, der Urin sowieso verhältnismäßig saurer ist, so kam die

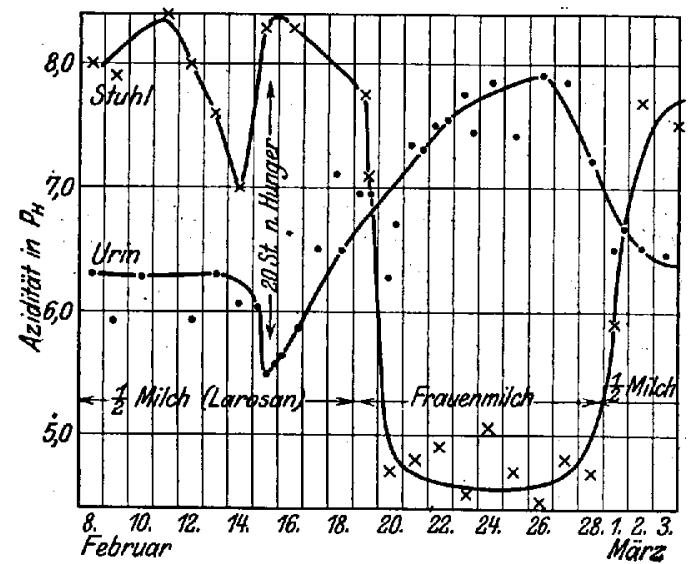

Fig. 4. Die Acidität des Stuhls und Uring bef Utbergang von Kuhmilch- zur Frauenmilchernährung. Fall 63: Frühgeburt Förster, 5 Mon., Gewicht $3100 \mathrm{~g}$.

Veränderung unregelmäßig und weniger übersichtlich zur Geltung (siehe Fall 3, 7 u. a.).

In Versuchen, in welchen die Versuchsbedingungen umgekehrt lagen als in den vorangegangenen, d. h. wo man von Kuhmilchmischungen zur Frauenmilchernährung überging, waren dieselben augenscheinlichen Umwälzungen bezüglich der Urin- und Stuhlacidität festzu-

stellen. Zeichnet man diese Verhältnisse graphisch, wie ich es auf. Fig. 4 gemacht habe, so kreuzen sich die Linien in ähnlicher Weise. Was aber die Lage der darstellenden Linien betrifft, so steht dieselbe hier in direktem Kontrast zu den auf Fig. 2 gezeichneten.

Dieses Verhalten des Stuhls und Urins bei Frauenmilch und Kuhmilchernährung ist bei gesunden Kindern mit normalen Stühlen vollkommen gesetzmäßig: bei Frauenmilch bewegt sich die Urinacidität oberhalb der Linie für die Stuhlacidität; bei Kuhmilchernährung ist das Verhältnis umgekehrt, wie auch aus den Versuchen Fig. 5, 6, 7, 8 und 9 klar hervorgeht. In allen diesen Versuchen war die Urinacidität bei Frauenmilchernährung bei Kindern

Verhalten bezüglich des Stuhles festgestellt. Seine Arbeit wird demnächst in dieser Zeitschrift erscheinen. 
im Alter von 4-5 Monaten $P_{\mathrm{H}}$ ca. 6,5-7,5, also bedeutend höher als in den eingangs erwähnten Versuchen bei jüngeren Frühgeburten.

Als Resü me e dieser Untersuchungen ist folgendes zu sagen: bei gleichbleibender Frauenmilchernährung ist die Acidität des Stuhles bei normalen Stühlen vom ersten Lebenstage an bis auf Monate hinaus ziemlich konstant $\left(P_{H} 4,6-5,2\right)$, während die Acidität des Urins unter gleichen Bedingungen mit zunehmendem Alter eine deutliche Steigerung zeigt. Bei Frühgeburten werden die bei Frauenmilchernährung üblichen Aciditätswerte des Urins von $P_{\mathbf{H}}$ ca, 6,5-7,5 meistens erst nach 2-4 Monaten erreicht, bei Neugeborenen schon in den ersten Wochen. Von dieser Zeit ab wird bei Frauenmilchernährung von einem gesunden Kinde ein Urin ausgeschieden, dessen Acidität meistens oberhalb des neutralen Punktes und Stühle, deren Acidität tief unterhalb desselben Punktes liegt. Bei Kuhmilchernährung liegen die Verhältnisse umgekehrt.

Es herrschen demnach unverkennbare Wechselbeziehungen bezüglich der wahren Reaktion gleichzeitiger Darm- und Nierenausscheidung. Wechselbeziehungen; die in hohem Maße durch Nahrungsveränderungen beeinflußt werden können.

Es fragt sich nun, in welcher Weise die Nahrungsveränderung diese Verschiebungen hervorrufen könnte. Sind es vielleicht irgendwelche sauren Produkte, die aus der Kuhmilch im Darmkanal entstehen, die dann im Körper aufgenommen und nur zum Teil neutralisiert ausgeschieden werden? Es können hierbei sowohl organische wie anorganische Säurebestandteile in Frage kommen. Nun wußte man durch Keller ${ }^{1}$ ) und Freund ${ }^{2}$ ), daß Phosphorsäure bei Kuhmilchernährung im Urin vermehrt vorkommt. Bei diesem vermehrten Gehalt an Phosphorsäure hatte Keller durch Titrationsmethode aber keine relative Vermehrung des primären Phosphates gefunden, das nur allein die Acidität erhöhend wirkt. Dagegen sprach eine Angabe von Sold i ${ }^{3}$ ), dafür, daß organische Säuren hierbei eine Rolle spielen könnten. Er berichtet, daß organische Säuren, d. h. flüchtige Fettsäuren in beträchtlichen Mengen, die außerdem bei verschiedenen Ernährungen größere Schwankungen zeigen, im Säuglingsharn auftreten. Dies veranlaßte mich, festzustellen, inwieweit organische Säuren, die in großen Mengen per os

1) Keller, loc. cit., S. 296 dieser Arbeit.

2) Freund, loc. eit., S. 296 dieser Arbeit.

3) Soldin, Zur Kenntnis der Darmfäulnis im Säuglingsalter bei verschiedenartiger Ernährung. Jahrb. f. Kinderheilk. 65, 292.1907. 
gegeben werden, tatsächlich imstande sind, die Aeidität des Urins zu steigern, und deswegen habe ich folgende Untersuchungen vorgenommen:

3. Einfluß der Acidität der Nahrung auf die wahre Reaktion des Urins.

Zunächst habe ich mehreren Kindern n-Essigsäure in Mengen von $1-3 \mathrm{ccm}$ pro $100 \mathrm{ccm}$ Frauenmilch gereicht. Die Acidität der Nahrung schwankte zwischen $P_{H}$ ca. 4,5-5,4, die der reinen Frauenmilch war $\mathrm{P}_{\mathrm{H}}$ ca. 6,8. Diese Nahrung bekamen kleine Frühgeburten im Alter von 2-3 Monaten. Der Urin wurde 2-3 mal täglich untersucht, es zeigte sich aber absolut kein Einfluß auf die Acidität des Urins. Dieselben Versuche wurden mit Milchsäure in gleichen Mengen und in gleicher Anordnung wiederholt. Die Werte der mit Säure versetzten Nahrung schwankten in Grenzen von $P_{H}$ ca. 4,4-5,5. Auch diese Versuche hatten ein negatives Ergebnis. Der Organismus scheint demnach die zugeführten Milch- resp. Essigsäuremengen glatt zerlegt zu haben. Dann versuchte ich noch größere Mengen von Säure zu reichen. Dies mußte ich aber, um Schädlichkeiten auszuschalten, in verschleierter Form tun. Ich habe deswegen die Essigsäure resp. Milchsäure gleichzeitig mit ihren Na-Salzen gegeben und zwar in folgendem Gemisch :

A.

$$
\frac{\text { n-Essigsäure }}{\text { n-Na-Acetat }} \frac{1 \text { Teil }=}{1 \text { Teil }=} 10,0 \mathrm{cem}
$$

und Frauenmilch . . . . . = $100,0 \mathrm{ccm}$

$P_{H}$ dieses Gemisches zeigte, wie physikalisch-chemisch vorauszusehen war, täglich ziemlich konstante Werte von $P_{H}$ 4,40:

B.

$$
\begin{array}{ll}
\mathrm{n} \text {-Milchsäure } & 1 \text { Teil }= \\
\hline \frac{\mathrm{n}}{2} \text {-Na-Lactat } & 4 \text { Teile }= \\
&
\end{array}
$$

und Frauenmilch . . . . . . = 100,0 $\mathrm{ccm}$

$P_{H}$ dieses Gemisches war ebenfalls wie oben konstant, $P_{H}$ ca. 4,6 (siehe Näheres über diese Versuche in den Protokollen, Fall 63, 24 und 25).

Die Kinder nahmen diese sauren Mischungen gern, zeigten absolut keine krankhaften Symptome, die Stühle blieben unverändert; bemerkenswert war nur eine auffallende Polyurie, die diese 2-3 tägigen Versuche noch mehrere Tage überdauerte. 
Bezüglich der Acidität des Urins bei dieser stark sauren Nahrung stellte sich in allen Versuchen folgendes heraus: die $\mathbf{P}_{\mathbf{H}}$-Wertedes Urins stiegen nach 24 Stunden unerwartet hoch, zeigten nach 2 Tagen oft Werte bis $P_{H} 8,50$, wie dies am besten aus Fig. 5 zu sehen ist.

Diese Befunde zeigen einwandfrei, $\operatorname{da} \beta$ im gesunden kindlichen Organismus sogar bei Frühgeburten sowohl Essigsäure wie Milchsäure glatt zerlegt werden, auch wenn sie in größeren Mengen eingeführt werden. Das unerwartete Verhalten des Urins: Steigen der Alkalescenz bei saurer Nahrung, ist darauf zurückzuführen, daß die Essigsäure- bzw. Milchsäurekomponente des Gemisches zerlegt wurden und das aus den Na-Salzen übrigbleibende Natri u m imstande war, die sauren Stoffwechselprodukte nicht nur normalerweise, sondern weit darüber zu neutralisieren.

Die Versuche zeigen einwandfrei, daß die flüchtigen Säuren, die im Darminhalt von manchen Autoren gefunden worden sind und denen Bahrdti) und Mitarbeiter eine große Rolle bei der Pathogenese der Verdauungsstörungen zuschreiben, in keiner Weise direkt als Säuren auf den intermediären Stoff wechsel Einfluß haben, weil sie eine aciditätsteigernde Wirkung vermissen lassen. Ihre Rolle bei den dyspeptischen Erscheinungen will ich nicht bestreiten, wenn auch meine Versuche dafür keinen

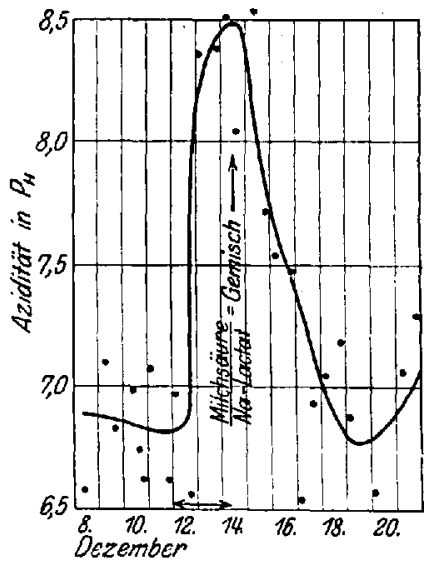

Fig. 5. EinfluB der, sauren Nabrung" (Milchsaure - Na - Lactat - Gemisch + Frauenmilch) auf die Aciditst des Urins. Fall 24: Fruhgeburt $B$ iging, 8 Tago alt, Gewicht $1900 \mathrm{~g}$. Anhaltspunkt geben können. $\mathrm{Da} \beta$ die Zufuhr von Essigsäure keinen Einfluß auf die Acidität ausübt, dafür sprechen auch die Versuche von Aron und $F r a n z^{2}$ ), die übrigens die Angaben von Soldin widerlegen. Diese Autoren haben flüchtige Fettsäuren im Säuglingsharn auch bei pathologischen Zuständen vermißt. Úber das Vorkommen von Milchsäure bei Säuglingen liegen meines Wissens bisher keine Angaben vor; nach den Untersuchungen,

1) Bahrdt, Zur Pathogenese der akuten Verdauungsstörung im Säuglingsalter. Verhandl. d. Gesellsch, f. Kinderheilk., Karlsruhe 1911, S. 62, und weitere Mitteilungen, Zeitschr. f. Kinderheilk. 1911-1913.

2) Aron u. Franz, Organische Säuren im .Säuglingsharn. Monatsschr. f. Kinderheilk. 12, 645. 1914. 
die man bei Erwachsenen ${ }^{1}$ ) angestellt hat, ist es aber wahrscheinlich, daß sie auch im Säuglingsurin normalerweise nicht vorkommt.

Der Vollständigkeit halber hätte ich noch Versuche mit anorganischen Säuren: Salzsäure und Phosphorsäure vornehmen müssen, um auch über die Wirkung des vermehrten Gehalts dieser Säuren in der Nahrung auf die Acidität des Urins Genaueres zu erfahren. Diese sind vorläufig ausgeblieben; es ist aber wahrscheinlich, daß sie eine Steigerung der Harnreaktion hervorrufen könnten, was auch vor kurzem bezüglich der Salzsäure von $\mathrm{Hassel} \mathrm{ba}_{\mathrm{ch}} \mathrm{c}^{2}$ ) bei Erwachsenen nachgewiesen worden ist. Inwieweit die Phosphorsäure, die ja bei Kuhmilchernährung vermehrt im Urin ausgeschieden wird, dieselbe Steigerung hervorruft, diese Frage ist

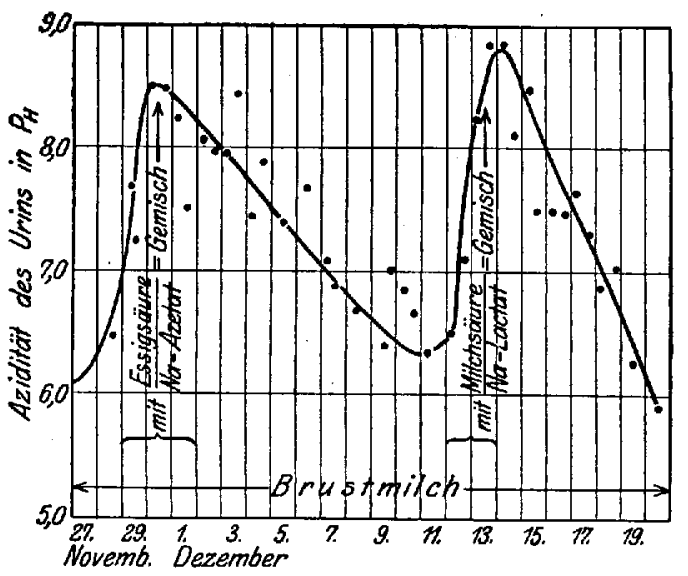

Fig. 6. Linfluß der ,sauren Nahrung" (Essigsăure-Na-Acetatresp. Milchsäure-Na-Lactat-Gemisch + Frauenmilch). Fall63;

Frühgeburt Förster, ca. 3 Mon., Gewicht $2000 \mathrm{~g}$. noch offen; es ist aber anzunehmen, daß die Aciditätssteigerungen, die wir bei Ernährung mit Kuhmilch im Urin festgestellthaben, wenigstens teilweise darauf zurückzuführen sind. Meine Absicht war aber nicht, tiefer in die einzelnen chemischen Faktoren hineinzublicken, die bei der Entstehung des acidotischen $\mathrm{Zu}$ standes in Frage kommen könnten, sondern ich hatte mir zur Aufgabe gestellt, nach allgemeingültigen acidotischen Merkmalen zu forschen. Ehe ich auf meine weiteren Versuche eingehe, möchte ich in diesem Zusammenhange noch besonders auf einen in den besprochenen Versuchen gemachten Befund hinweisen, der nicht nur eine pädiatrische, sondern eine allgemein physiologische Bedeutung hat. Michaelis ${ }^{3}$ ) hat nämlich in seiner „Wasserstoffionenkonzentration" S. 108 gesagt, daß ,die Wasserstoff-

1) Irisawa, zit. nach Porges, U̇ber die Milchsäure im Blute und im Harne. Wiener klin. Wochenschr. 24, 1147. 1911.

2) Hasselbalch, Ammoniak als physiologischer Neutralitätsregulator. Biochem. Zeitschr. $74,18.1916$.

3) Michaelis, Die Wasserstoffionenkonzentration. Berlin 1914 (Springer). 
zahl des Harns selten viel unter $10^{-7}$ und selbst nach Zufuhr großer Mengen von Alkalien allerhöchstens auf die $\left(H^{*}\right)$ des Blutes sinkt". Ich habe oft bei gesunden Kindern bei Frauenmilchernährung $\mathrm{P}_{\mathrm{H}}$ bis 7,92 (siehe Fall 63), nach dem Hunger sogar noch höhere Werte $P_{H}$ bis 8,56 (Fall 61), gefunden, während $P_{H}$ des Blutes normalerweise nur $P_{H} 7,4-7,6$ zeigt. Auch Hasselbalch hat spontanerweise beim

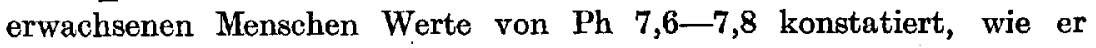
in seiner jüngst veröffentlichten Arbeit ${ }^{1}$ ) berichtet. Bei der Säurenahrung traten oft Werte bis $P_{H} \cdot 8,50$ auf. Dies alles zeigt, daß die Alkalescenz des Blutes keineswegs die höchste Grenze für die Harnreaktion nach der alkalischen Richtung hin bildet.

\section{B. Die $\mathrm{CO}_{2}$-Regulationsbreite des Blutes mit zunehmendem Alter. (Protokolle: $27-48$ u. a. Fälle.)}

Uber die Bedeutung dieses Begriffes weise ich auf den diesbezüglichen Abschnitt bei der Beschreibung der Methode hin und gehe gleich auf die Versuche näher ein. Ich habe die $\mathrm{CO}_{2}$-Regulationsbreite des Blutes im ganzen bei 12 Neugeborenen und Frühgeburten teils im Nabelschnurblut, bei der Geburt, teils in den ersten Stunden oder Tagen nach der Geburt untersucht. Es zeigte sich hier eine auffallende Abweiehung von den bei gesunden Erwachsenen und bei etwas älteren Kindern gefundenen Werten. Während die aktuelle Reaktion, $d$. h. der regulierte Wasserstoffexponent, bei allen untersuchten Objekten innerhalb der ziemlich engen Grenzen von $\mathrm{P}_{\mathrm{H}} \mathbf{7 , 2 5 - 7 , 5 2}$ schwankte, wich derGrund-Wasserstoffexponent bei Neugeborenen, einerlei, ob ausgetragene oder frühgeborene, deutlich von dem bei Erwachsenen und beim älteren Kinde gefundenen $a b$. Die durchschnittlichen Werte für den Grund-Wasserstoffexponenten bei Neugeborenen waren $P_{\mathbf{H}}$ ca. 8,0 (die Grenzwerte: 7,86 und 8,21; dieser letzte und höchste Wert wurde im Nabelschnurblut gefunden), dieselben in den anderen Gruppen $\mathrm{P}_{\mathrm{HI}}$ ca. 8,35. Rechnet man aus den Zahlen die durchschnittlichen Prozentwerte für die $\mathrm{CO}_{2}$-Regulationsbreite, so bekommt man ca. $60 \%$ (Grenzwerte $46-77 \%$ ) bei Ne ugeborenen. Berücksichtigt man nicht die im Nabelschnurblute, sondern nur die im Blute von einigen Stunden bis zu einigen Tagen alten Neugeborenen gefundenen Werte, so wird die Prozentzahl für die $\mathrm{CO}_{2}$-Regulationsbreite hier noch etwas niedriger

1) Hasselbalch, Ammoniak als physiologișcher Neutralitätsregulator. Biochem. Zeitschr. 74, 27. 1916. 
(ca. $55 \%$ ). Dies im Gegensatz zu den Erwachsenen, wo der entsprechende Durchschnittswert bei ca. 90\% und die Grenzwerte zwischen 84-100\% lagen. Diese Verhältnisse werden am besten aus der Fig. 7 ersichtlich.

Diese niedrigen Werte müssen als ein besonderes Charakteristic u m des Neugeborenenblutes bezeichnet werden und sprechen eine deutliche Sprache dafür, daß im Neugeborenenblute die regulatorischen Fähigkeiten viel kleiner sind als im Blute im späteren Lebensalter. Die Zahlen zeigen ja deutlich, daß die nicht flüchtigen, sauren Komponenten des Neugeborenenblutes auf Kosten der $\mathrm{CO}_{2}$ vermehrt sind. Diese Vermehrung kann nicht auf irgendeinen Hungerzustand (der ja auch in demselben Sinne, wie später gezeigt wird, wirkt) zurückgeführt werden, denn sie war schon bei der Geburt und auch schon nur
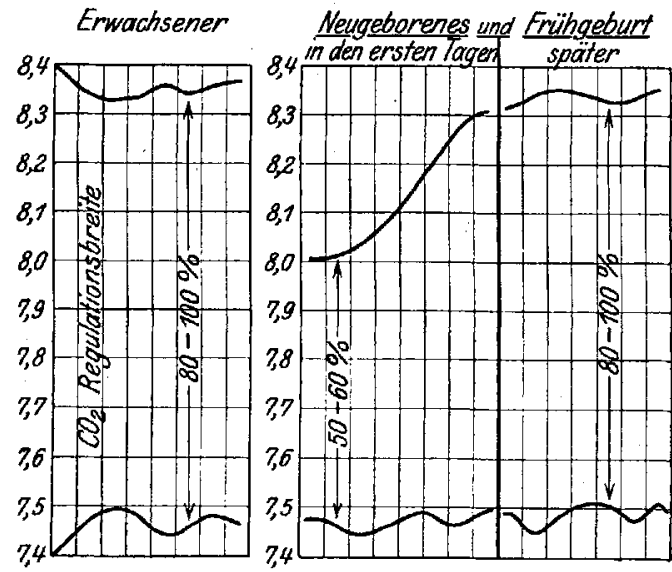

Fig. 7. $\mathrm{CO}_{\mathrm{a}}$-Regulationsbreite bei Neugeborenen und älteren Săuglingen und beim Erwachsenen. Obere Linie = Grund-Wasserstoffexponent $\mathbf{P}_{\mathbf{H}}$. Untere Linle $=$ Regulierter Wasserstoffexponent $P_{H^{*}}$. einige Stunden nach der Geburt deutlich und ausgesprochen nachweisbar. Man kann demnach ohne weiteres sagen, daß das Neugeborenenblutim Vergleich zum Blute in späteren Lebens - Abschnitten als sicher acidotisch zu bezeichnen ist. Wie schnell dann das Blut, ob in Tagen oder in Wochen nach der Geburt, allmählich dieselben regulatorischen Fähigkeiten erreicht, die man im späteren Säuglingsalter und nachher trifft, kann ich nicht sagen. Z. B. bei einer Frühgeburt im Alter von 18 Tagen war die $\mathrm{CO}_{2}$-Regulationsbreite $72 \%$, bei anderen Frühgeburten im Alter von 1-2 Monaten schwankte sie von $72-92 \%$, in einem Falle, bei einer 2 Monate alten Frühgeburt, war sie sogar $118 \%$ (siehe Fall 39, 44). Man könnte demnach vielleicht sagen, daß im Alter von 1-3 Monaten sich die $\mathrm{CO}_{2}$ - $\mathrm{Re}$ gulationsgrenze bei Frühgeburten ca. um $80 \%$ herum bewegt, d. h. in den unteren Grenzen der bei den Erwachsenen gefundenen Werte.

Nebenbei möchte ich auch hier erwähnen, daß ich für die $\mathrm{CO}_{2^{-}}$ Regulationsbreite in der Gravidität, während der Novak, Leim- 
dörfer, Porges ${ }^{1}$ ) Hasselbalch und Gammeltoft ${ }^{2}$ ) einen herabgesetzten $\mathrm{CO}_{2}$-Alveolardruck festgestellt haben, die sie aus diesen und anderen Gründen als einen acidotischen Zustand betrachten, in 3 Fällen Werte von $84-85 \%$ gefunden haben. Bei einem derselben 9 Stunden nach der Entbindung sogar nur einen Wert von $71 \%$; also auch insgesamt Werte, die an der unteren Grenze der $\mathrm{CO}_{2}$-Regulationsbreite beim normalen Menschen liegen. Diese ist gewissermaßen eine Kontrollprobe, die, soweit diese wenigen Fälle überhaupt einen Schlußsatz zulassen, dafür spräche, daß wir in der $\mathrm{CO}_{2}$-Regulationsbreite einen Indicator für den acidotischen Zustand sehen können.

\section{Die $\mathrm{O}_{2}$-Dissoziationskurve des Blutes mit zunehmendem Alter. (Protokolle Fall 49-52 u. a.)}

Es war nun erforderlich und angezeigt, weitere Beweise dafür zu erbringen, daß das Blut von einem neugeborenen Kinde, einerlei ob frühgeboren oder ausgetragen, tatsächlich acidotisch ist, wie die obigen Versuche es wahrscheinlich machten. Nach meiner Meinung konnte hierüber am besten die $\mathrm{O}_{2}$-Dissoziationskurve des Neugeborenenblutes Aufschlu $\beta$ geben. Wie bei der Besprechung der Methode schon erwähnt, weicht die $\mathrm{O}_{2}$-Dissoziationskurve bei Urämie und Diabetes beträchtlich von der Kurve des gesunden Erwachsenen ab. Barcroft spricht ja die Meinung aus, daß dies

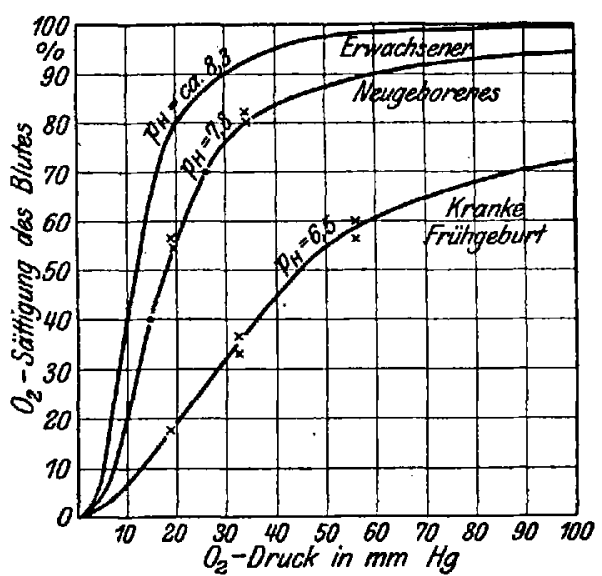

Fig. 8. $O_{g}$-Dissoziationskurve des $O_{2}$-freien Blutes beim gesunden Erwachsenen, beim Neugeborenen und bei einer kranken Frihgeburt.

Verhalten durch Anhäufung von sauren Produkten, die bei Urämie und Diabetes, welche er beide für acidotische Zustände hält, im Blute kreisen, verursacht wird. Weiterhin wußte man schon durch $\left.B o h r^{3}\right)$, daß der $\mathrm{CO}_{2}$-Partialdruck des Blutes einen gewaltigen Einfluß auf den Verlauf der Dissoziationskurve des

1) Novak, Leimdörfer u. Porges, Über die $\mathrm{CO}_{2}$-Spannung des Blutes in der Gravidität. Zeitsehr. f. klin. Med. 75, 301. 1912.

2) Hasselbalch u. Gammeltoft, Die Neutralitätsregulation des graviden Organismus. Biochem. Zeitschr. 68, 206. 1915.

3) Bohr, loc. cit., S. 291 dieser Arbeit. 
Blutes hatte. Barcroft ${ }^{1}$ ) und sein Schüler Mathison ${ }^{2}$ ), zeigten dann, daß andere Säuren dasselbe tun und sprachen die Vermutung aus, daß $\mathrm{CO}_{2}$ und andere Säuren ihren erniedrigenden Einfluß auf den Ver-

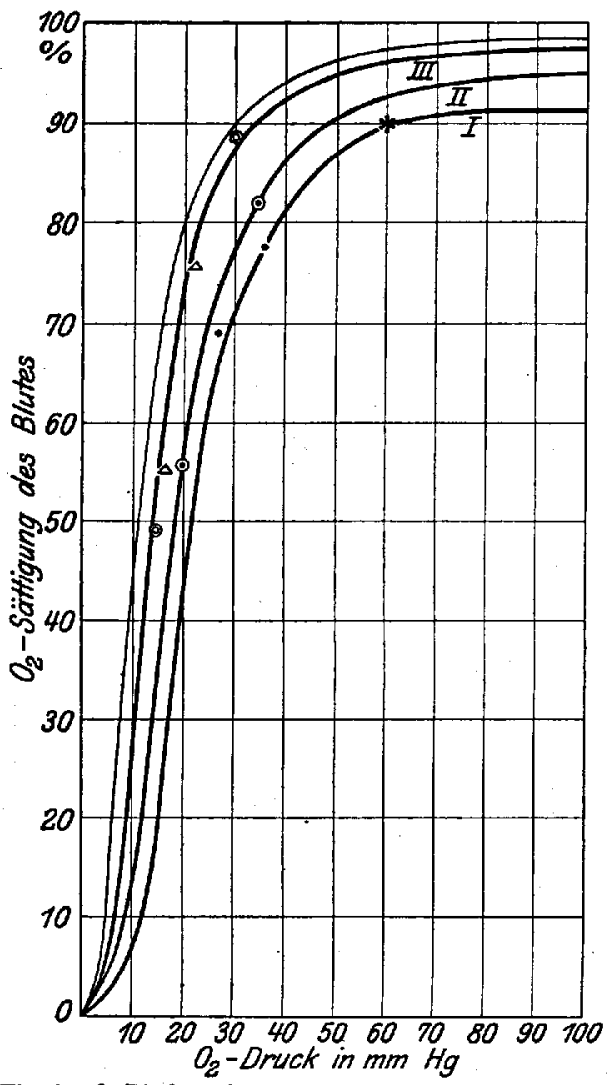

Fig. 9. $\mathrm{O}_{2}$-Bindungskurve des $\mathrm{CO}_{3}$-freien Blutes be zunehmendem Alter bei $38^{\circ}$. Fall 49 (Kind Jacoby), Brustkind. $I=$ Nabelsehnurblut, II = Blut im Alter von 5 Tagen, III = Blut im. Alter von 2 Monaten. Dunne Linie $=$ Normalkurve des Erwachsenen, $*=$ ein aus der Formel $\frac{\boldsymbol{Y}}{100}=\frac{K \cdot x^{n}}{1+K \cdot x^{n}}$ bereohneter Punkt. $\Delta=$ ans dem Blute der Mutter J, eine Woche vor der Entbindung bestimmte Punkte. lauf der Dissoziationskurve je nach dem Gehalt an freien $\mathbf{H}-$ Ionen ausüben. Rona und $\mathrm{mir}^{3}$ ) ist dann gelungen, den Beweis dafür zu bringen, daß die Dissoziationskurve des Blutes tatsächlich in ganz prägnanter Weise von der Größe der Wasserstoffzahldesselben abhängig ist. Nun hatte ich in den obigen Versuchen gefunden, daB die GrundWasserstoffzahl des Blutes bei Neugeborenen größer ist als im Blute beim späteren Alter $\left(P_{\mathrm{H}}\right.$ ca. 8,0 gegenüber 8,4$)$. Es war demnach schon von vornherein sehr wahrscheinlich, daß die Dissoziationskurve des $\mathrm{CO}_{2}$ - freien Neugeborenenblutes einen niedrigeren Verlauf annehmen müßte als im späteren Alter. Und dies bestätigte sich dann bei den Versuchen.

In Fig. 8 sind diese Verhältnisse graphisch dargestellt.

Wir sehen auf der Kurve, daB die $\mathrm{O}_{2}$-Sättigung beim Neugeborenen bis $20 \%$ kleiner ist als dieselbe beim Erwachsenen unter entsprechenden $\mathrm{O}_{2}$-Partialdrucken. Die Normalkurve des Erwachsenen, bei $\mathrm{CO}_{2}$-Partial-

1) Barcroft, loc. eit., S. 287 dieser Arbeit.

2) Mathison, Journ. of Physiol. 43, 347. 1911.

a) Rona u. Ylppö, Der Einfluß der Wasserstoffionenkonzentration auf die Dissoziationskurve des Hämoglobins. Biochem. Zeitschr. 76, 187. 1916. 
druck $=0$, habe ich aus der Barcroftschen Monographie entnommen. Als Grundlage zu der Kurve des Neugeborenen liegen eigene Bestimmungen bei 3 Kindern vor (Fall 49, 50 und 51). Die Kurve der kranken Frühgeburt (Fall 56) habe ich auf die Fig. 8 nur eingetragen, um die Differenzen noch anschaulicher zu machen.

Bei einem Kinde war ich imstande, die $\mathrm{O}_{2}$-Dissoziationskurve 1. gleich nach der Geburt im Nabelschnurblut, 2. im Alter von 5 Tagen und 3. im Alter von 2 Monaten zu bestimmen (Fall 49). Die betreffenden Dissoziationskurven sind auf Fig. 9 gezeichnet. Wie ersichtlich, ist die Kurre des Nabelschnurblutes am tiefsten, dann folgt die Kurve des Blutes am 5. Lebenstage, sie weicht auch noch immerhin, bis $15 \%$, von der Normalkurve ab; die Kurve des Blutes im Alter von 2 Monaten dagegen fällt aber schon annähernd zusammen mit der dünnen Linie, die die Dissoziationskurve des Erwachsenen angibt. Demnach ist das Blut bei der Geburt am stärksten acidotisch, am 5. Tage auch noch deutlich acidotisch und im Alter von 2 Monaten wohl als normal zu betrachten. Was die Kurve vom 5. Lebenstage betrifft, so könnte der Einwand gemacht werden, daB zu dem niedrigen Verlauf der Dissoziationskurve außer etwaiger acidotischer Beschaffenheit des Neugeborenenblutes auch noch eine gewisse Hungerwirkung beiträgt. Um den Einwand zu entkräften, bringe ich folgende klinische Daten: Das Kind wog bei der Geburt $2700 \mathrm{~g}$, war ein ausgetragenes debiles Kind. Physiologische Abnahme bis zum dritten Lebenstage $150 \mathrm{~g}$, von da an langsame Zunahme, so daß das Gewicht bei der Untersuchung am 5. Lebenstage wieder 2620 g erreicht hatte. Die Nahrungsmengen betrugen am ersten Tage 0 , am 2. Tage $90 \mathrm{~g}$, am 3 . Tage $170 \mathrm{~g}$, am 4. Tage $230 \mathrm{~g}$, am 5. Tage, dem Untersuchungstage, $260 \mathrm{~g}=70$ Kalorien pro $\mathrm{kg}$ Körpergewicht. Hieraus geht hervor, daß es sich höchstens um etwas knappe Ernährung, aber nicht um einen Hungerzustand am Tage der Blutentnahme handelte.

Dies alles genügt schon, um mit Sicherheit behaupten zu können, daß bei der Geburt und in den ersten Lebenstagen ein gewisser acidotischer Zustand existiert. Ich halte mich schon nach allem obigen berechtigt, hier von einer gewissen ,acidotischen Konstitution" des Neugeborenen- (Frühgeborenen-) Organismus zu sprechen. Diese Eigentümlichkeit des Neugeborenen-Organismus kommt noch durch die folgenden Untersuchungen besonders stark zur Geltung. 
D. Reaktion der Gewebe und des Herzblutes einschl. der Bestimmung der $\mathrm{CO}_{2}-$ Regulationsbreite und der Dissoziationskurven bei Frühgeburten und jungen Säuglingen (Protokolle 53-59 u. a.).

Später wird gezeigt, daß die Acidität des Blutes im toxischen $\mathrm{Zu}$ stande oft etwas zunimmt und daß die Zunahme im agonalen Stadium besonders rapid vorwärts geht. Gleichzeitig mit dem Größerwerden der regulierten Wasserstoffzahl vergrößert sich auch die Grund-Wasserstoffzahl. Meistens nimmt die Grund-Wasserstoffzahl schneller zu als die regulierte Wasserstoffzahl, und daraus folgt, da $B$ die $\mathrm{CO}_{2}$-Regulationsbreite immer kleiner wird, bis sie bisweilen nur ca. 10\% beim Eintreten des Exitus ausmacht. Dieses starke Annähern der Grund-Wasserstoffzahl an die regulierte Wasserstoffzahl habe ich in erster Linie bei Frühgeburten beobachtet. Diese Erscheinung kann nur als Ausdruck von besonders starker Vermehrung der nicht flüchtigen, sauren Stoffwechselprodukte erklärt werden, die imstande gewesen sind, beinahe die ganze $\mathrm{CO}_{2}$ aus dem Blute zu verdrängen. So z. B. fand ich im Fall 54 einer 18 Tage alten Frühgeburt von $1150 \mathrm{~g}$ im Blute, das unmittelbar vor dem Exitus durch Sinuspunktion gewonnen wurde, Grund-Wasserstoffzahl = 6,58 und regulierte Wasserstoffzahl $=6,45$, woraus sich eine $\mathrm{CO}_{2}$-Regulationsbreite von $13 \%$, ergibt. Hierbei ist noch besonders zu betonen, daß das Blut im wahren Sinne des Wortes schon im Leben stark sauer war, etwa hundertfach saurer als normalerweise.

Es ist klar, da $B$ in einem solchen Zustande das Leben nicht länger bestehen kann. Die lebenswichtigen oxydativen und fermentativen Funktionen können in so saurem Medium wie das Blut hier ihre Wirksamkeit nicht mehr normalerweise ausüben, und außerdem ist ja die Aufnahme der $\mathrm{CO}_{2}$ aus den Geweben ins Blut praktisch nicht mehr möglich.

Die Werte, die man im Herzblute eben verstorbener Frühgeburten findet, zeigen, daß die Säuerung des Blutes bei ihnen beim Eintreten des Todes sehr hochgradig ist. Mit einer einzigen Ausnahme (Fall 55, Kind Maud), wo der regulierte Wasserstoffexponent $P_{\mathrm{H}} 7,01$ war, sind die Werte für ihn besonders sauer und schwanken von $\mathbf{P}_{\mathbf{H}}$ ca. 6,2-6,7, ebenfalls die entsprechenden Werte für den GrundWasserstoffexponenten, die sich in den Grenzen von $P_{\mathbf{H}}$ ca. 6,5-7,3 bewegten. Diese Werte, die bedeutend größer sind als im entsprechenden Blute beim älteren Kinde, mit Ausnahme einiger toxischer Fälle, die später besprochen werden, beweisen, daß das Frühgeburtenblut, das schon im gesunden Zustande acidotische Merkmale zeigte, beim 
Herannahen des Todes auch in besonderer Weise zur Ubersäuerung neigt. Auch Pfau ndler war, wie oben (S. 277) erwähnt, schon aufgefallen, daß das Herzblut von Frühgeburten auffallend sauer ist.

Besonders interessante Verhältnisse zeigte das Herzblut von Frühgeburten bezüglich der Dissoziationskurve. In allen Fällen nahm sie einen auffallend niedrigen Verlauf, so daß die prozentuelle $\mathrm{O}_{2}$-Sättigung manchmal kaum $50 \%$ von der bei Erwachsenen unter entsprechenden $\mathrm{O}_{2}$-Partialdrucken gefundenen betrug (s. Fall 53, 56, 57, 59 u. a.). Die Verhältnisse gehen am besten aus der Fig. 8 hervor, wo die unterste Linie den Verlauf der Dissoziationskurve vom Fall 56 darstellt. Es handelt sich hier, wie aus dem Protokolle näher ersichtlich, um eine ca. 1 Monat alte Frühgeburt, die mit 2650g ohne irgendwelche akutentzündliche Erscheinungen an Lues zugrunde gegangen ist. Es ist ja ganz klar, daß das Blut, dessen $\mathrm{O}_{2}$-Bindung so stark von dem normalen abweicht, nicht mehr imstande ist, dem Organismus die nötige Menge $\mathrm{O}_{2}$ abzugeben und daß der Tod als Folge von lang- Fig. 10. Die Reaktion des Blutes und der Gewebe bel samer Erstickung betrachtet

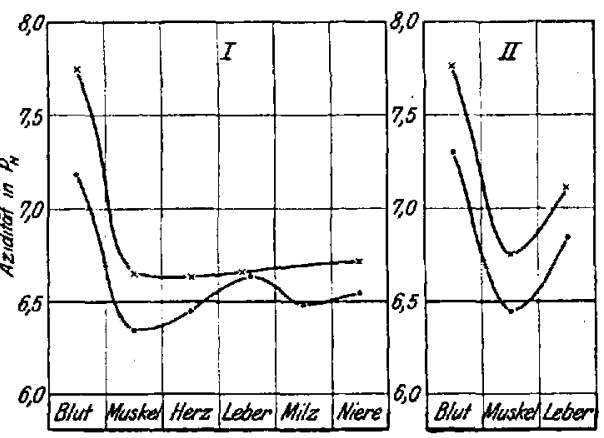
werden kann (s. die. Dissoziationskurve vom Fall 59 auf Fig. 18).

Was dann die Reaktion der verschiedensten Gewebe bei den Frühgeburten betrifft, so sind da ebenfalls auffallend starke Säuerungen zu konstatieren. Ich habe leider zum Vergleich keine Werte vom gesunden Säugling, der plötzlich ohne vorhergehende Erkrankung durch Unglücksfall usw. gestorben wäre, und mußte deshalb die Vergleichswerte für normale Verhältnisse aus Tierversuchen heranziehen.

Ich habe zunächst bei zwei gesunden Meerschweinchen unter besonderen Vorsichtsmaßregeln, um Selbstsäuerung zu viermeiden, die Acidität der Organe bestimmt (s. Genaueres Protokoll Fall 67-68). Die gefundenen Werte, die innerhalb der Grenzen fallen, die auch von Michaelis und Kramszty $\mathbf{k}^{1}$ ) bei gesunden Meerschweinchen gefunden worden sind, zeigt am anschaulichsten die graphische Darstellung auf Fig. 10.

1) Michaelis u. Kramsztyk, loc. cit., S. 284 dieser Arbeit (Methoden). 
Wir sehen, daß hier alle Werte, mit Ausnahme der für die Muskulatur, oberhalb des Wertes $P_{H} 6,5$ liegen. Diesen Grenzwert von 6,5 habe ich dann auf allen folgenden Figuren, die die Acidität der Gewebe darstellen, eingetragen, um einen bestimmten Vergleichspunkt bei der Beurteilung der pathologischen Werte zu haben.

Bei Frühgeburten, die ich unmittelbar nach dem Exitus sezieren konnte, stellte sich nun heraus, daß die Aciditätswerte für die Gewebe, sogar die für das Blut, meistens unterhalb dieser Vergleichslinie lagen. Besonders hochgradige Verschiebungen nach der sauren Richtung hin wurden im Falle 54 beobachtet. Hier handelt es sich um eine kleine Frühgeburt mit einem Geburtsgewicht von $1150 \mathrm{~g}$, die am 2. Lebenstage in die Klinils gebracht wurde. Das Kind neigte trotz Wärmewanne stark zur Untertemperatur, zeigte auch sonst allerlei Zeichen von Lebensschwäche, bekam am neunten Tage dünne Stühle, die trotz Frauenmilch-

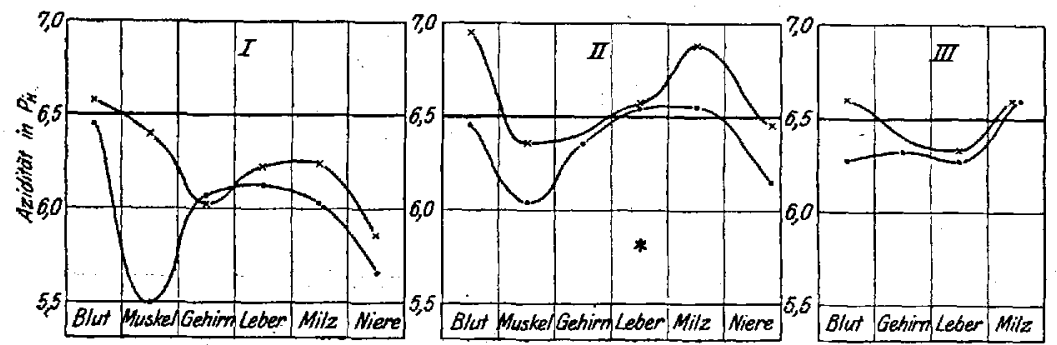

Fig. 11. Die Reaktion des Herzblutes und der Gewebe bei drei Fríhgeburten. Untere Linie: Reaktion der frischen Organe; obere Linie: Reaktion der gekochten Organe. I Fall 54, II Fall 53 , * Reaktion der Galle III Fall 56.

ernährung nicht besser wurden, und starb toxisch nach langandauernder Agonie mit einem Gewicht von $960 \mathrm{~g}$ im Alter von 18 Tagen (s. Fig. 11, I). Die Werte für die Muskulatur liegen auffallend tief; der für den frischen Muskel bei ca. $P_{H} 5,5$, der für den gekọchten Muskel bei ca. $P_{\mathrm{H}} 6,40$. Die Werte für Gehirn, Leber, Milz liegen in dem Gebiete ca. $\mathbf{P}_{\mathbf{H}} 6,0$ bis 6,2, die für die Niere bei ca. $P_{\mathrm{H}} 5,6-5,8$.

Diese Werte sind die sauersten, die ich gefunden habe. Es nähern sich ihnen nur die Befunde von Fall 70 (Fig. 28 II) und 71 (Fig. 26), wo es sich um ein $2 \frac{1}{2}$ und ein 8 Monate altes Kind handelt, die an schwerer Intoxikation zugrunde gegangen sind.

In einem anderen Falle 53 handelt es sich um ein 2 Wochen altes Kind, das im ca. 8. Schwangerschaftsmonate geboren und bis 2 Tage vor dem Tode ganz gesund war. Am 12. Lebenstage bekam das Kind ein phlegmonöses Erysipel am Halse und starb am 14. Tage plötzlich 
an Glottisödem. Hier war die Säuerung des Gewebes nicht so hochgradig wie in dem ersten Falle. Im dritten Falle dieser Fig. 11 sind die Organwerte von einer Frühgeburt, die im Alter von ca. 1 Monat an Lues zugrunde ging (s. Genaueres Fall 56), dargestellt. Diese Worte liegen mit Ausnahme des für die Milz ebenfalls unterhalb der Vergleichslinie und was besonders interessant: das $\mathrm{Blut}$, sowohl $\mathrm{CO}_{2}$-haltiges wie $\mathrm{CO}_{2}$-freies, ist beinahe ebenso sauer wie die Organe. Hier bestand also gar kein Säuregefäll vom Gewebe nach dem Blute hin, was die Ausscheidung von sauren Produkten aus den Körpergeweben ins Blut hätte ermöglichen können.

Bei den anderen Frühgeburten ist dieses Säuregefäll (Differenz in den Acidiätswerten zwischen Blut und Geweben) gewiß noch vorhanden, es ist aber überall auffallend gering im Vergleich zu den Befunden bei allen anderen Kindern, die im späteren Lebensalter verstorben sind.

Auch bei einer Totgeburt, bei der ich die Organe untersuchen zu können in der Lage war, war der augenfälligste Befund ein fehlendes Säuregefäll (s. Fig. 12, Protokoll 73).

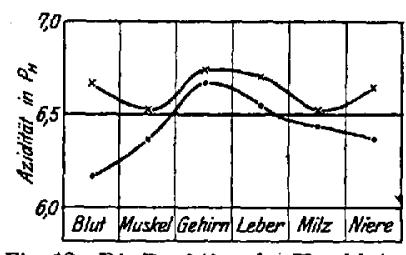

Fig. 12. Die Reaktion des Herzblutes und der Gewebe bei einer Totgeburt.

Hier handelt es sich keineswegs u m $\mathrm{CO}_{2}$-Anhäufung im Blute, sondern um eine Vermehrung von anderen sauren Stoffwechselprodukten, wie das die starksauren Werte für den regulierten Wasserstoffexponenten $P_{H}$ 6,17 und für den Grundwasserstoffexponenten $P_{H} 6,68$ belegen. Wenn auch die Werte für Gehirn- und Leberacidität dicht oberhalb der Vergleichslinie liegen, so bildet dies doch in Anbetracht der stark sauren Werte des Blutes keinen Gegenbeweis, daß es sich auch hier um abnorme Anhäufung von sauren Stoffwechselprodukten handelt. Und im Zusammenhang mit den anderen Befunden, die deutlich für die acidotische Konstitution des Neugeborenen-Organismus sprechen, kann wohl diese vorläufig einzig dastehende Untersuchung über die Acidität der Organe bei Totgeburten auch als Beitrag gelten.

Gegenüber diesen 7 Fällen, bei denen die Reaktion des Blutes und der Gewebe in gleichem Sinne für eine besondere acidotische Neigung des Organismus spricht, steht als einzige Ausnahme der Fall 58. Da waren bei einer Frühgeburt von $1100 \mathrm{~g}$, die im Alter von 2 Tagen an Lebensschwäche starb, im Gegensatz zu den vorigen die Werte für die Reaktion der Gewebe und des Blutes bedeutend weniger sauer. Blut $\mathrm{P}_{\mathrm{H}} 6,52$ resp. $\mathrm{P}_{\mathrm{H}} 7,35$, ungekochter Muskel $\mathrm{P}_{\mathrm{H}} 6,67$, gekochter 
Muskel $P_{H} 7$ 19. Die Werte für andere Organe schwankten zwischen ca. $P_{\mathbf{H}} 7,0-7,3$. Diese sind überhaupt die höchsten $P_{\mathbf{H}^{-}}$Werte, die ich für die Organe bei Säuglingen gefunden habe. Eine Erklärung für diesen Ausnahmebefund kann ich nicht abgeben und muß mich damit begnügen, ihn hiermit zu konstatieren.

Was sonst die Bestimmung der Reaktion im Gewebe eines verstorbenen Kindes betrifft, so bin ich mir wohl bewußt, daß dabei postmortale Säuerungprozesse in Frage kommen. Deswegen können die gefundenen Werte keinen sicheren Maßstab für die Werte im lebenden Organismus abgeben. Sie sind aber bei allen Kindern, die auch späterhin untersucht wurden, unter gleichen zeitlichen usw. Bedingungen ausgeführt, und die Abweichungen voneinander sind natürlich miteinander vergleichbar. Ubrigens ist es bemerkenswert, daß man oft im Gewebe eines verstorbenen Kindes, wie wir später sehen werden, Werte findet, die mit denen bei den gesunden Tieren, bei denen die Bestimmungen gleich nach der Tötung unter besonderen Vorsichtsmaßregeln vorgenommen wurden, zusámmenfallen. Dies spricht ja dafür, daß die spontane Säuerung in kurzer Zeit nur bis zu einem gewissen Grade rasch vor sich geht und dann beim längeren Stehen nur ganz langsam weiter geht. Dies habe ich auch bei meinen Versuchen oftmals beobachtet. Das Gesagte berechtigt mich, die gewonnenen Werte als Vergleichswerte anzuführen und Schlußsätze bezüglich der betreffenden untersuchten Objekte hieraus zu ziehen.

\section{Untersuchungen im Hunger.}

A. Die Einwirkung des Hungers auf den Säuglingsorganismus im Sinne der „Acidose“6 (Untersuchungen wie A-D, Kap. IV).

Die Acidität des Urins und Stuhls im Hunger beim künstlich und natürlich ernährten Säugling.

Durch verschiedene Untersuchungen an Tieren [zuletzt Elias und $\mathrm{Kolb}^{1}$ ) u. a.] und bei Erwachsenen [Barcroft ${ }^{2}$ ), Hasselbalch $\left.{ }^{3}\right)$ u.a.] ist nachgewiesen, daß der Hungerzustand in gewisser Hinsicht ein acido-

1) Elias u. Kolb, Über die Rolle der Säure im Kohlenhydratstoffwechsel. II. Mitt.: Über Hungerdiabetes. Biochem. Zeitsehr. 52, 331. 1913.

2) Bareroft, loc. cit., S. 287 dieser Arbeit.

3) Hasselbalch, Neutralitätsregulation und Reizbarkeit des Atemzentrums in ihren Wirkungen auf die $\mathrm{CO}_{2}$-Spannung des Blutes. Biochem. Zeitschr. 46, 403. 1912 . 
tischer Zustand ist. Langstein und Meyer ${ }^{1}$ ) hatten bei Säuglingen konstatiert, daß im Hunger die Menge der Acetonkörper und der Ammoniakkoeffizient im Urin beträchtlich steigen und auf Grund dieser Befunde den Schlußsatz gezogen, daß der Hunger beim Säugling acidoseerzeugend wirkt. Ich habe im folgenden versucht, an der Hand der oben besprochenen, für einen acidotischen Zustand charakteristischen Merkmale, bei hungernden Säuglingen diesbezügliche Untersuchungen anzustellen.

Für die ersten Hungerversuche wählte ich zwei etwas debile, sonst aber ganz gesunde und gut gedeihende Kinder. Das erste Kind, Fall 60 (Kind Jacoby), war am Anfang des ersten Versuches ca. 4 Monate alt

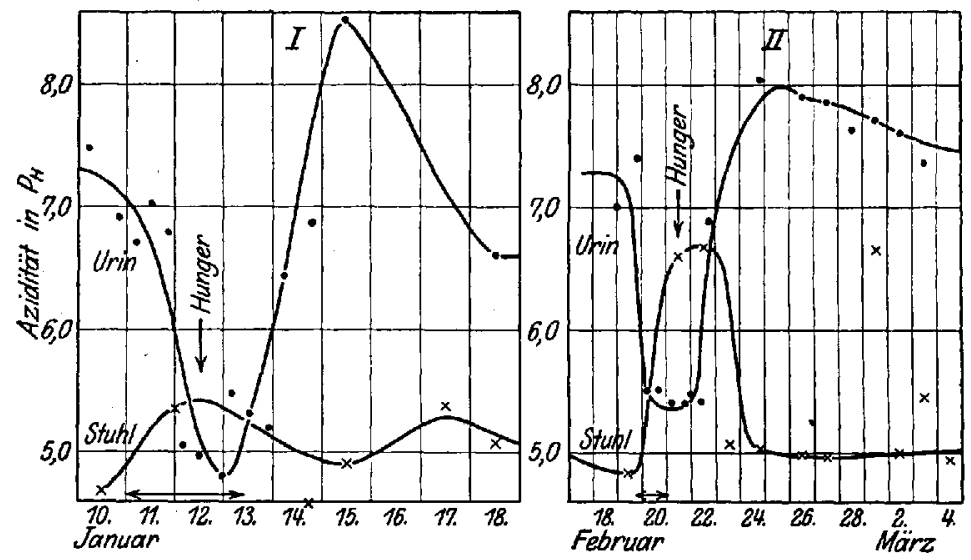

Fig. 13. Einwirkung des Hungers auf die Aciditht des Stuhls und Urins beim "Brustmilchkinde" Fall 61 (Kind Lux).

und wog $4740 \mathrm{~g}$. Es hatte in den letzten $1 \frac{1}{2}$ Monaten künstliche Ernährung $(1 / 2$ Milch) bekommen und wird hier im Gegensatz zu dem zweiten Versuchskinde als "Flaschenkind" bezeichnet. Das letztere, Fall 61 (Kind Lux), war eine Frühgeburt von ca. $1500 \mathrm{~g}$ Geburtsgewicht, die bisher nur Frauenmilch bekommen hatte und damit recht gut ge: diehen war, so daß es am Anfang des Versuches, im Alter von ca. 4 Monaten, $3000 \mathrm{~g}$ wog.

In dem ersten Hungerversuche, der bei beiden Kindern 2 Tage und 7 Stunden dauerte, bekamen die Kinder $1 / 2$ Ringerlösung $+1 / 2$ Tee in gleichen Mengen wie sie vorher Nahrung getrunken hatten, sonst aber nichts. Utber das klinische Verhalten s. Genaueres in den Protokollen Fall 60 und 61. Die Acidität des Stuhls und Urins wurde einige

1) Langstein u. Meyer, loc. eit., S. 278 dieser Arbeit. 
Tage vor dem Hungerversuch, während des Hungers und nach demselben untersucht, ebenfalls die aktuelle Reaktion des Blutes am Anfang und am Ende des Versuchs festgestellt.

Bezüglich des Blutes stellte sich bei beiden Kindern die interessante Tatsache heraus, daß die aktuelle Reaktion trotz des verhältnismäßig langdauernden Hungers sich absolut in kei ner nennenswerten Weise verändert hatte. Im Falle 60, „Flaschenkind“, war der regulierte Wasserstoffexponent vor dem Hunger $P_{H} 7,53$, am Ende desselben $P_{\mathbf{H}} 7,51$. Im Falle 61 , „Brustmilchkind“, zeigte er vor dem Hunger $P_{H} 7,48$, am Ende $P_{H} 7,53$. Um so deutlicher war aber der Einfluß des Hungers auf die Acidität des Urins, wiederum weniger deutlich auf die des Stuhls, wie dies aus Fig. 13 I und 14 I hervorgeht.

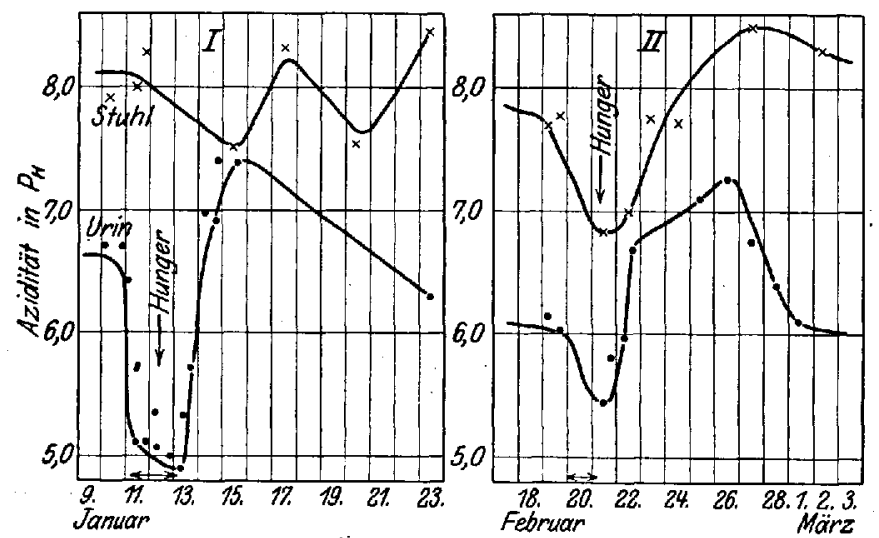

Fig. 14. Einwirkung des Hungers auf die Acidität des Stuhls und Urins beim „Flaschenkind" Fall 60 (Kind Jacoby).

Wir sehen aus den beiden Figuren, daß der Urin in beiden Fällen gegen Ende des ersten Hungertages eine stark vermehrte Acidität zeigte; die $\mathrm{P}_{\mathrm{H}}$-Werte waren so tief oder so klein, wie wir sie kaum bei den Frühgeburten normalerweise in den oben angeführten Versuchen gefunden haben. Die Acidität hielt sich während des Hungers unverändert ziemlich hoch ( $\mathrm{P}_{\mathbf{H}}$ klein!), um nach der Nahrungszufuhr am folgenden Tage wieder zu fallen. Die $\mathbf{P}_{\mathrm{H}}$-Werte des Urins sind in beiden Fällen und, wie aus den folgenden Versuchen hervorgeht, regelmäßig immer einige Tage nach dem Hunger böher als vor dernselben, was möglicherweise als irgendwelche Uberkompensationserseheinungen des Organismus beim Versuch, saure Hungerprodukte auszuscheiden, aufzufassen ist. Bei den gleichen Kindern wurden ca. 1 Monat später neue 
Hungerversuche vorgenommen. Diesmal dauerte die Hungerperiode 40 Stunden, während der die Kinder überhaupt nichts zu trinken bekamen. Sie waren also im absoluten Hunger. Bezüglich des klinischen Verhaltens dieser Kinder während des Versuches verweise ich auf die Protokolle Fall 60 und 61, erwähne hier nur, daß beim Fall 61 (Kind Lux) schon nach 24stündigem Hunger eine Temperatursteigerung bis $37,9^{\circ}$ auftrat. Diese erhöhte Temperatur hielt sich etwa unverändert bis das Kind Nahrung bekam, dann fiel sie wieder bis zur Norm auf $37^{\circ}$ zurück. Das Kind zeigte sonst immer vor und nach dem Hungerversuche eine auffallende Monothermie, so daß ich diese Fiebersteigerung als Durstfieber auffassen will. Dies ist um so wahrscheinlicher, als Müller ${ }^{1}$ ) auch von Fiebersteigerungen bei gelegentlichem Durst bei jungen Säuglingen berichtet.

Bezüglich der Urinund Stuhlacidität zeigte sich genau das gleiche Verhalten wie in den erstenHungerversuchen, wodieKinder $1 / 2$-Ringerlösung zum Trinken bekamen (s. Fig. 13 II, 14 II).

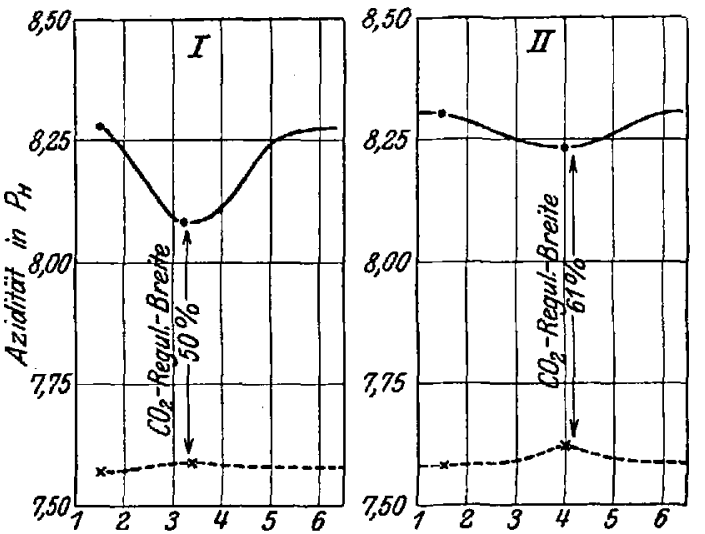

Ebenfalls waren in diesen Versuchen keine

Fig. 15. Verhalten der $\mathrm{CO}_{\mathbf{3}}$-Regulationsbreite beim 40 stundigen absoluten Hunger. I* "Flaschenkind", II "Brustmilchkind" (Fall 60 und 61).

Veränderungen in der aktuellen Reaktion des Blutes festzustellen. Ich habe aber diesmal auch die $\mathrm{CO}_{2}$-Regulationsbreite bestimmt und da eine große Versehiebung konstatiert. Beim Flaschenkinde war die Regulationsbreite vor dem Hunger $=70 \%$, am Ende des Hungers nur 50\%. Bei dem Brustkinde $72 \%$ vor dem Hunger, nachher $61 \%$. Diese Schwankungen bezüglich der $\mathrm{CO}_{2}$-Regulationsbreite sind auf Fig. 15 I; "Flaschenkind", und 15 II, "Brustmilchkind", graphisch dargestellt. Bei beiden Kindern ist demnach eine sichere und deutliche Verschmälerung der $\mathrm{CO}_{2}$-Regulationsbreite infolge des $\mathrm{Hungers}$ nachzuweisen.

Um die großen Verschiebungen bezüglich des Grund-Wasserstoff1910.

1) Müller, E., Durstfieber bei Säuglingen. Berl. klin. Wochenschr. 47, 673. 
exponenten noch besser zu veranschaulichen, habe ich Fig. 16 gezeichnet. Diese zeigt, daß beim „Brustmilchkind“ die Verschiebung bedeutend kleiner ist als beim „Flaschenkinde", was ja dafür spricht, daß bei dem "Flaschenkinde" die Vermehrung der sauren, nicht flüchtigen Stoffwechselkomponente, während des 40 stündigen Hungers eine bedeutend größere gewesen ist als bei dem "Brustmilchkinde“. Schon diese Versuche zeigen, daß neben der Steigerung der Urinacidität bei $m$ Hunger auch im Blute Veränderungen vor sich gehen, die bezeichnend für einen acidotischen Zustand sind.

In weiteren Hungerversuchen, die ich bei zwei kleineren Frühgeburten unternommen habe, zeigten sich im Prinzip die gleichen Er-

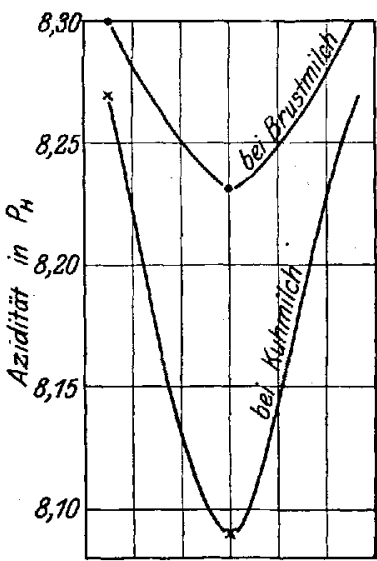

Fig. 16. Verhalter des GrundWasserstoffexponenten bei 40 stündigem Hunger bei einem, Flaschenkinde" und bei einem ,Brustmilch kinde ${ }^{a}$, Fall 60 und 61 . scheinungen, nur waren hier die Ausschläge besonders im Blute bedeutend größer. Die eine Frühgeburt, Fall 63 (Kind Förster), war mit $1500 \mathrm{~g}$. geboren, hatte von Anfang an bis zum Alter von ca. 2 Monaten Kuhmilchmischungen bekommen, dann ca. I Monat lang Frauenmilch und in den letzten $1 \frac{1}{2}$. Monaten vor dem Versuch wieder nur $1 / 2$ Milch (Larosan) mit 4\% Soxhletzucker. Ich betrachte dieses Kind dem folgenden gegenüber demnach als „Flaschenkind". Das Kind war gut gediehen, hatte regelmäßig zugenommen and machte einen verhältnismäßig kräftigen Eindruck. Der erste Versuch wurde im Alter von 4y/2 Monaten vorgenommen. Das Kind wog damals $2740 \mathrm{~g}$. Der Hunger dauerte $2^{1} / 2$ Tage, währenddessen bekam es nur $1 / 2$ Ringerlösung und 1/2 Tee. Derselbe Versuch wurde 10 Tage später wiederholt. Im Alter von $51 / 2$ Monaten wurde bei demselben Kinde ein dritter Hungerversuch vorgenommen, bei dem es sich aber im Gegensatz zu den vorangegangenen um absoluten Hunger handelte, der 20 Stunden dauerte.

Genau dieselben drei Versuche wurden bei einer gleichaltrigen, aber viel kleineren Frühgeburt, Fall 62 (Frühgeburt Köhler), vorgenommen. Das Geburtsgewicht dieses Kindes betrug nur $960 \mathrm{~g}$, die Gewichtszunahme war regelmäßig gut, das Kind machte aber im Gegensatz zu dem vorigen einen wenig lebenskräftigen und sehr schlaffen Eindruck. Es bẻkam über die Versuche hinaus Frauenmilch und wird aus diesem 
Grunde als „Brustmilchkind" bezeichnet. Beim Beginn des ersten Versuches, im Alter von etwas über 4 Monaten, betrug das Gewicht nur $2300 \mathrm{~g}$. Die Veränderungen in der Acidität des Stuhls und Urins in diesen Versuchen gehèn aus den Fig. 17, 18, 19 (s. auch Fig. 4) hervor.

Hier wie in den obigen Versuchen wird der Urin schon innerhalb der ersten 24 Stunden auffallend sauer, in beiden Fällen $\mathrm{P}_{\mathrm{H}}=$ ca. $5,4-5,6$, und hältsich dann unverändert bis zum Endedes Hungers. Nach der Nahrungszufuhr fängt die Urinreaktion nach ca. 24 Stunden an, sich rasch wieder nach der alkalischen Richtung zu verschieben. Am zweiten und den folgenden Tagen fand ich nach der Nahrungszufuhr die Urinreaktion bedeutend alkalischer als sie je vor dem Hunger war. Beim Brustmilchkinde fand ich da Werte $\mathrm{P}_{H}=$ ca. 8,2, während vor dem Hunger die Urinacidität um $P_{H}$ ca. 7,0 herum sich bewegte. Beim Flaschenkinde war ebenfalls nach der Nahrungszufuhr eine starke Steigerung der Werte für

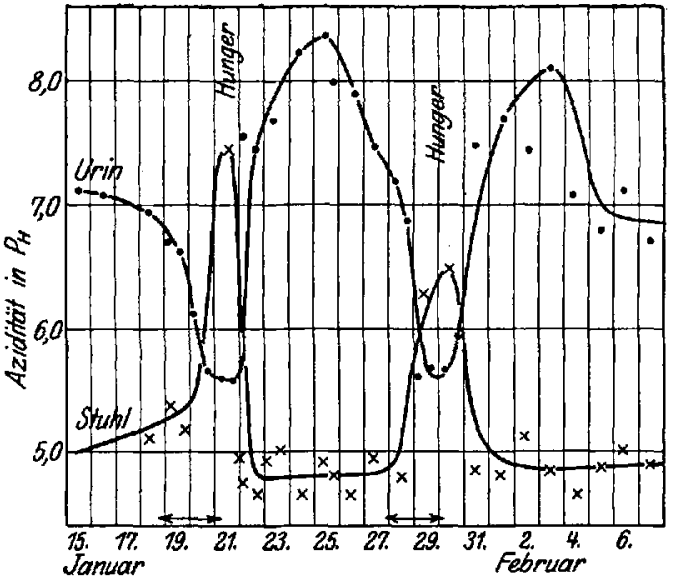

Fig. 17. Ijie Eiuwirkung des Hungers auf die Stuhl- und Harnaciditat bei einem "Brustmilchkinde", Fall 62 (Frthgeburt Köhler). Dauer des Hungers $21 / 2$ Tage, nur $1 / 2$ Ringerlösung $+1 / 2$ Tee.

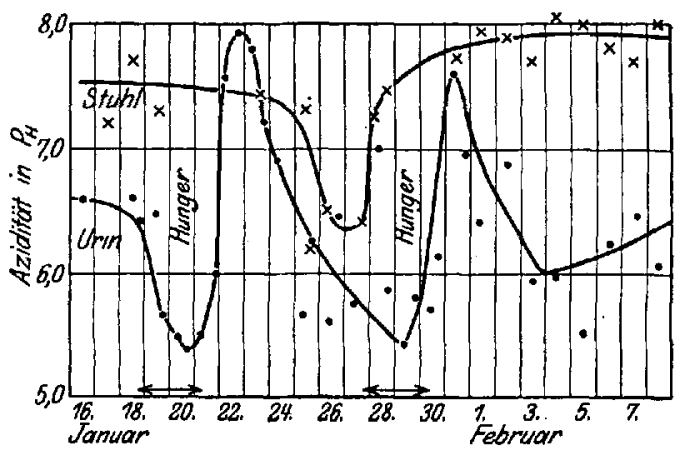

Fig. 18. Die Elnwirkung des Hungers auf die Stuhl- und Harnacidität bei einem, "Flaschenkinde", Fall 63 (Kind Förster). Dauer des Hungers $2 \%$ Tage, nur 1/2 Ringerlösung $+1 / 2$ Tee. $\mathrm{P}_{\mathrm{H}}$ der Urinreaktion zu konstatieren; am höchsten bis ca. $P_{H} 8,0$, während die Acidität des Urins bei demselben Kinde ca. um $P_{H}=6,5$ vor und nach dem Hunger schwankte. Diese Werte für die Urinacidität bei meinem Brustmilchkinde während des Hungers entsprechen ${ }^{\urcorner}$denen bei demselben Kinde (Frühgeburt!) im ersten Monat (siehe Tabelle S. 295 dieser Arbeit). Die 
Werte für Stuhlacidität zeigten keine nennenswerte Veränderung bei dem Flaschenkinde, wohl weil der Stuhl schon vor dem Hunger alkalisch war, $P_{H}=$ ca. 8,0. Beim Brustmilchkinde war dagegen bezüglich der Stuhlreaktion eine deutliche Ferschiebung nach der alkalischen Richtung hin konstatierbar.

Interessant waren die Veränderungen des Blutes infolge

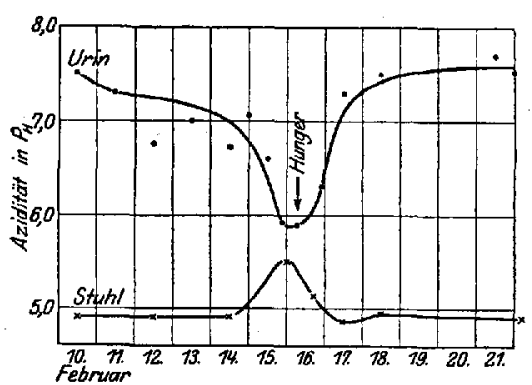

Fig. 19. Die Einwirkung des 20 stindigen absoluten Hungers bel elnem ,Brustmilchkinde", Fall 62 (Fribgeburt KöhIer). des Hungers. Bei meinem Brustmilchkinde zeigte sich im Gegensatz zu dem Flaschenkinde und zu den Befunden bei den beiden oben erwähnten Kindern, daß das Bl ut eine deutlich merkbare, wenn auch kleine Steigerung der aktuellen Reaktion in allen Hungerversuchen angenommen hatte. Im ersten Versuch war der regulierte Wasserstoffexponent vor dem Hunger $P_{H} 7,57$, am Ende des Hungers $P_{H} 7,33$; im zweiten vor dem Hunger $P_{\mathbf{H}} 7,50$, am Ende des Hungers $P_{\mathbf{H}} 7,41$, drei Tage nach dem Versuche wieder $P_{H} 7,50$. Im dritten Versuche vor dem

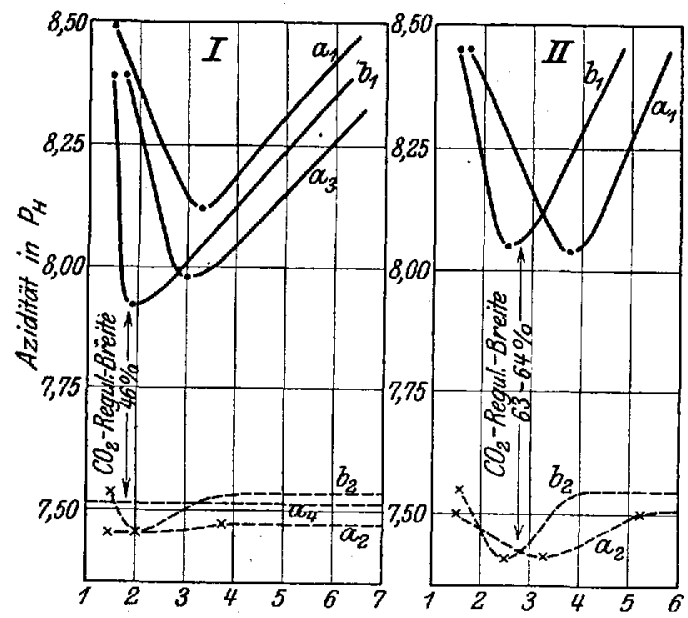

Fig. 20. EinfluB des Hungers auf die $\mathbf{C O}_{2} \cdot$ Regulationsbreite des Blutes bei zwei Frïhgeburten. I = Fall 68, Frühgeburt Förster, ca. $2800 \mathrm{~g}$, "Flaschenkind", $41 / \mathrm{g}$ bis 5 Monate alt. Obere Linie: Grund-Wasserstoffexponent; untere Linie : regulierter Wasserstoffexponent $a_{1}$ bis $a_{4}$ bei zwel $21 / 2$ tägigen Hungerversuchen, $b_{1}$ und $b_{2}$ bei 20 stündigem, absolutem Hunger. II = Fall 62, Frühgeburt Köhler, Gewicht ca.2300 g, „Brustmilchkind“. Linien und Zeichen wie bel $\mathrm{I}$.
Hunger $\mathrm{P}_{\mathrm{H}} 7,55$, am Ende des Hungers $\mathrm{P}_{\mathrm{H}}$ 7,41. Dies ist wohl das erste Mal, daß man beim Menschen eine Blutacidose mit Steigerung der $\left(\mathrm{H}^{*}\right)$, infolge von Hunger festgestellt hat. Ich muß besonders betonen, daß es sich hier, wie oben und in dem Protokoll 62 erwähnt, um eine sehr kleine Frühgeburt handelte, die nur dank besonders sorgfältiger Pflege am Leben erhalten werden konnte. Das Kind war schon in der Zeit der Versuche sehr blaß und 
schlaff, zeigte aber sonst keine krankhaften Erscheinungen. Was die $\mathrm{CO}_{2}$-Regulationsbreite bei dem Kinde betrifft, so zeigte sie auch in den beiden letzten Hungerversuchen, in denen sie bestimmt wurde, eine starke Verschmälerung von $95-63 \%$, resp. $90-64 \%$; Verhältnisse, die ich auf Fig. 20 II graphisch dargestellt habe.

Bei dem „Flaschenkinde" war wiederum keine Veränderung in der aktuellen Reaktion bezüglich des Hungers festzustellen. Dagegen war eine große Verschmälerung der $\mathrm{CO}_{2}$-Regulationsbreite in allen drei Hungerversuchen nachweisbar. Im ersten Versuch war die $\mathrm{CO}_{2}$-Regulationsbreite am Ende des Hungers $45 \%$, gegen $87 \%$ vor dem Hunger; im zweiten Versuche $66 \%$ gegen $104 \%$; im dritten Versuche $46 \%$ gegen $85 \%$. Die Verengung der $\mathrm{CO}_{2}$-Regulationsbreite ist, wie aus diesen Zahlen und aus Fig. $20 \mathrm{I}$ ersichtlich, bei diesem Flaschenkinde wiederum größer als die des obigen „Brustmilchkindes", Fall 62. Dies ist insofern ein unerwarteter Befund, weil bei dem „Brustmilehkinde" der Hunger sogar die aktuelle Reaktion, die der Körper sonst mit besonderer Hartnäckigkeit konstant aufrechterhält, nach der sauren Richtung hin verschieben konnte. Wenn man aber daran denkt, daß bei dem ersten „Flaschenkinde“, Fall 60, gegenüber dem ersten „Brustmilchkinde“, Fall 61, auch eine gröBere Verengung der $\mathrm{CO}_{2}$-Regulationsbreite in den Hungerversuchen auftrat, so fragt man sich unwillkürlich, ob da nicht eine spezielle Wirkung der vorangegangenen Kuhmilchnahrung in Frage kommen könnte.

Schlossmann und Murschhauser $\left.{ }^{2}\right)^{2}$ ) haben nun in ihren Stoffwechseluntersuchungen bei hungernden Säuglingen nachgewiesen, daß die unnatürlich ernährten Kinder im Gegensatze zu den natürlich ernährten Kindern im Hunger vielmehr Aceton und $\beta$-Oxybuttersäure ausscheiden. Nach ihnen scheidet z. B. das Brustkind am dritten Hungertage nur $1 / 3$ soviel von Aceton und $\beta$-Oxybuttersäure aus wie das unnatürlich ernährte Kind. Diese Untersuchungen sprechen ja deutlich dafür, daß das unnatürlich ernährte Kind im Hungerzustande bedeutend mehr saure Stoffwechselprodukte, die für Acidosis besonders charakteristisch gehalten werden, bildet und ausscheidet. Wenn auch der Zustand des Kindes, wie es am besten mein Fall 62 zeigt, eine große Rolle beim Auftreten der acidotischen Merkmale

1) Schlossmann, u. Murschhauser Einfluß der vorangegangenen Ernährung auf den Hungerstoffwechsel. Biochem. Zeitschr. 53, 273. 1913.

2) Schlossmann u. Murschhauser, Stoffwechsel des Säuglings im Hunger. Biochem. Zeitschr. 58, 493 . 1913/14. 
während des Hungers spielt, so spielt auch nach allem Obigen die vorangehende Nahrung eine besonders eminente Rolle hierbei.

Diese meine Hungerversuche zeigen demnach, daß der $\mathrm{Hunger}$ bei jedem Säugling zu einer Acidodis führt, und zwar ist der Grad der Acidosis gemessen an der Hand der $\mathrm{CO}_{2}$-Regulationsbreite des Blutes bei künstlich ernährtem Kinde größer als beim Brustmilchkinde, ebenfalls bei einem jungen Kinde größer als bei einem älteren Kinde. Unter Acidosis verstehe ich hier und wie auch sonst eine Vermehrung der organischen, möglicherweise auch anorganischen Säuren und deren Salze im Organismus, speziell im Blute. Acidosis kann eine sog. ,echte" Acidosis sein mit vermehrter $\left(\mathrm{H}^{*}\right)$ des Blutes oder eine verschleierte, bei der die aktuelle Reaktion des Blutes normal gefunden wird, die $\mathrm{CO}_{2}$-Regulationsbreite aber infolge der Anhäufung von abnormen sauren Produkten verschmälert ist.

\section{B. Die Einwirkung des Hungers auf den Tierorganismus.}

(Protokolle 64-66.)

Um noch einen genaueren Einblick in die Wirkung des Hungers, bezüglich acidotischer Veränderungen zu bekommen, habe ich zwei Hungerversuche bei gesunden Kaninchen vorgenommen. Zuerst ließ ich ein kräftiges, ausgewachsenes Kaninchen von $2880 \mathrm{~g}$ Gewicht 7. Tage lang (Fall 64) hungern. Bei Beginn des Hungers bestimmte ich die $\mathrm{CO}_{2}$-Regulationsbreite, sie war $63 \%$, nach eintägigem Hunger sogar etwas erhöht $69 \%$, am 5 . Tage $59 \%$, dann erst trat eine schnellere Verschmälerung der $\mathrm{CO}_{2}$-Regulationsbreite ein, und am 7. Tage war sie nur $36 \%$. Das Tier wurde dann getötet und die Organe mit möglichster Vorsicht und Schnelligkeit, um die Selbstsäuerung zu verhindern, zur Bestimmung der Gewebsreaktion benutzt. Es stellte sich dabei heraus, daß trotz der im Blute nachgewiesenen sicheren Vermehrung der $\left(\mathrm{H}^{*}\right)$ (vor dem Hunger war nämlich die regulierte Wasserstoffzahl $P_{H}=7,53$, nach dem Hunger aber nur $P_{H}=7,28$ ) doch keine besondere Vermehrung der Wasserstoffionen in den Organextrakten nachzuweisen war.

Alle Werte für die Organacidität; sowohl für die gekochten wie für die ungekochten Organe, sind größer als der oben besprochene Grenzwert $P_{\mathrm{H}} 6,5$.

Ich hoffte dann, daß ich beim jungen Kaninchen möglicherweise Hunger eine nachweisbare Säuerung der Organe hervorrufen könnte und ließ ein jüngeres Kaninchen von $2080 \mathrm{~g}$ Gewicht 10 Tage lang hungern. 
Auch hier war wiederum eine sichere Blutacidosis mit vermehrter $\left(\mathrm{H}^{\prime}\right)$ schon vom 4 . Hungertage an zu konstatieren. Gleichzeitig wurde auch die $\mathrm{CO}_{2}$-Regulationsbreite inmer schmäler und betrug am Ende des Versuches nur $43 \%$, gegenüber $80 \%$ beim Beginn des Versuches. Die aktuelle Reaktion des Blutes hatte sich von $P_{H}$ 7,38 am Anfang des Versuches bis $P_{\mathrm{II}} 7,07$ verschoben, also deutlich nach der sauren Richtung hin. Aber die Organe zeigten trotzdem keine saurere Reaktion als die Organe von einem erwachsenen gesunden Kaninchen, bei dem ich in gleicher Weise unter Berücksichtigung der obigen Vorsichtsmaßregel (wie aus den Protokollen genauer ersichtlich) die Bestimmungen ausgeführt habe. Das Verhalten der Organreaktion bei diesen Kaninchen ist auf Fig. 21 graphisch dargestellt. Da zeigt sich deutlich, daß während

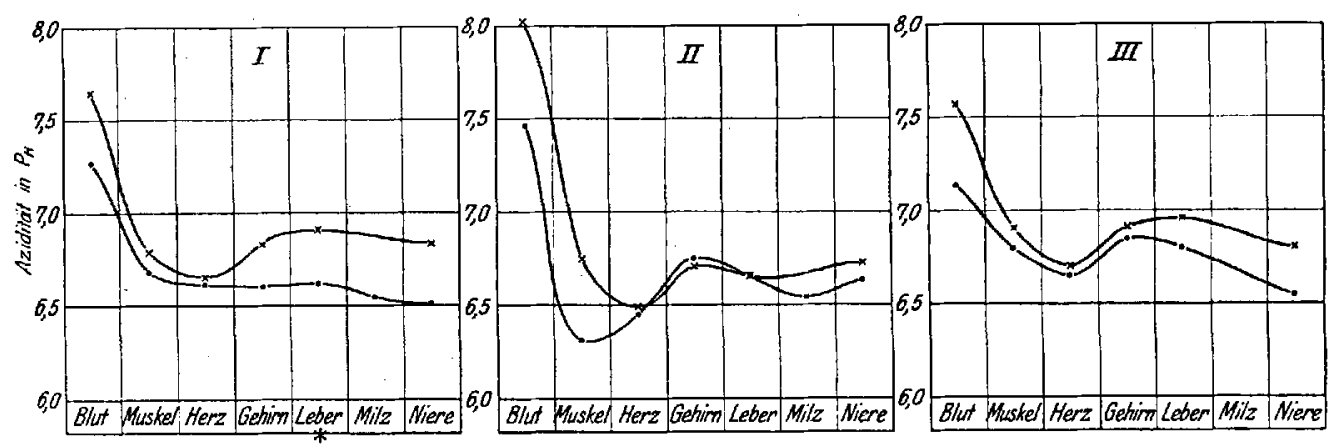

Fig. 21. Reaktion des Blutes und der Gewebe bei elnem gisunden und bei zwei hungernden Kaninchen. $I=$ Hunger von 7 Tagen, II $=$ Hunger von 10 Tagen.

bezüglich des Blutes große Unterschiede zwischen dem hungernden und gesunden Kaninchen vorhanden sind, sich die Reaktion der verschiedenen Gewebe bei allen beiden gleich verhält. Damit ist aber keineswegs gesagt, daß das Gewebe während des Hungers nicht saurer geworden wäre. Daß dies der Fall gewesen ist, beweist ja das Sauerwerden des Blutes in den beiden Hungerversuchen. Die Erklärung mag wohl darin liegen, daß die spontane Säuerung, die trotz aller Vorsichtsmaßregeln doch beim Töten der Tiere eingetreten ist, die im Leben sicher vorhanden gewesenen Differenzen bezüglich der Gewebsreaktion beim gesunden und hungernden Kaninchen ausgeglichen hat.

Meine beiden Kaninchen zeigten also im Hunger bezüglich der Blutreaktion viel größere acidotische Veränderungen als die oben besprochenen Säuglinge. Bei beiden war eine echte Blutacidosis mit vermehrter (H) infolge von Hunger aufgetreten. Das- 
selbe hat seinerseits auch $\mathrm{Hasselbalch}{ }^{1}$ ) beim hungernden Kaninchen beobachtet. Es scheint demnach ein gewisser Unterschied zwischen Kaninchenorganismus und dem menschlichen Organismus zu existieren. Das Kaninchen neigt demnach leichter zur Acidosis als der Mensch. Und interessant ist auch an dieser Stelle auf die alten Versuche von Gäthgens ${ }^{2}$ ) und Salkowski ${ }^{3}$ ) u. a. ${ }^{4}$ ) hinzuweisen, die ja schon nachgewiesen hatten, daß das Kaninchen bei künstlicher Säurezufuhr viel leichter als andere Tierarten (Hund) unter typischen SäureintoxikationsSymptomen zugrunde geht.

\section{VI. ,Acidose"6-Untersuchungen bei Intoxikation.}

Die vorangehenden Untersuchungen beziehen sich in der Hauptsache auf gesunde Säuglinge. Bei diesen wurde festgestellt, daß der Neugeborenen- und Frühgeborenenorganismus in den ersten Lebenstagen deutlich acidotisch ist. Ebenfalls wurde gezeigt, daß der Hunger beim gesunden Säugling einen acidotischen Zustand hervorruft, dessen Intensität von dem Zustande des Kindes und von der vorangehenden Nahrung abhängig ist.

Die jetzt folgenden Untersuchungen beschäftigen sich mit kranken Säuglingen, speziell mit Säuglingen, die klinisch das Bild der Intoxikation zeigten. Ich habe bei diesen Kindern unter denselben Prinzipien und Methoden wie oben nach acidotischen Merkmalen gesucht. Uber die Resultate dieser Untersuchungen will ich in derselben Reihenfolge, die ich mir bei den vorigen vorgeschrieben hatte, berichten:

\section{A. Die wahre Reaktion des Urins und des Stuhls bei Intoxikation.}

Nach allem Vorangehenden war es zu erwarten, daß die Reaktion des Urins sich im sauren Gebiete bewegen würde. Ist doch die Intoxikation schon wegen der meistens damit verbundenen heftigen Durchfälle, die die Nahrungsresorption stark beeinträchtigen, mit einem Hungerzustande gewissermaßen identisch. Und dazu kommt noch,

1) Hasselbalch, loc. cit., S. 312 dieser Arbeit.

2) Gäthgens, Zur Frage der Ausscheidung freier Säuren durch den Harn. Centralbl. f. d. med. Wissensch. 10, 833. 1872.

s) Salkowski, Über die Möglichkeit der Alkalienentziehung beim lebenden Thier. Virchows Archiv 58, 1. 1873.

4) Bostock, Gertrude, Zur Kenntnis der Säureintoxikation. Zeitschr. f. physiol. Chemie 84, 468. 1913. 
daß wir therapeutisch die Nahrungszufuhr besonders knapp bemessen. Was wieder die Stuhlreaktion betrifft, so hatte ich ja bereits gezeigt, daB sie in erșter Linie von der Qualität der Nahrung abhängig ist, so daB alle Kuhmilchmischungen bei normalen Stühlen eine alkalische Stuhlreaktion hervorrufen, im Gegensatze zur Frauenmilch, bei der die Reaktion sich im sauren Gebiete bewegt. Weiterhin ist die Stuhlreaktion in hohem Grade von der Beschaffenheit der Stühle abhängig, in dem Sinne, daß die wahre Reaktion der Stühle immer saurer wird, je dünner dieselben sind. Dies sowohl bei Frauenmilch wie bei Kuhmilch. Alles dies macht es wahrscheinlich, daß man bezüglich der Stuhlreaktion bei Intoxikation keine Gesetzmäßigkeit erwarten durfte. Und so war es auch, wie aus den Protokollen 69-90 ersichtlich ist. Ich gehe hier nicht näher auf die Zahlen ein, sondern erwähne nur, daß in den Fällen, wo viel Schleim und Eiter im Stuhl ausgeschieden wurde, die Reaktion sogar der dünnen Stühle verhältnismäßig wenig sauer, oft sogar alkalisch war (von $P_{H} 5,6-7,0$ ).

Was die Acidität des Urins betraf, so war dieselbe ziemlich konstant $P_{\mathrm{H}} 5,2-5,7$, solange der Intoxikationszustand dauerte und die Nahrungszufuhr gering war. Die untere Grenze der Urinreaktion liegt demnach bei Intoxikationen i m sa uren Gebiete, ziemlich genau in demselben sauren Gebiete wie beim Hunger. Es scheint hier bei ca. $\mathbf{P}_{\mathbf{H}}$ 5,2 die unterste Grenze für die Urinacidität beim Säugling zu liegen. Die Niere des Säuglings scheint auch in den schwersten pathologischen Zuständen diese Grenze nur selten überschreiten zu können. Nur bei einem Falle (21) habe ich im Urin $\mathrm{P}_{\mathrm{H}}=4,91$, bei einem Atrophiker gefunden. Interessant ist aber, daß ich bei den Frühgeburten gar nicht so selten Werte ca. $\mathrm{P}_{\mathrm{H}} 5,0$ getroffen habe, was dafür spricht, daß die Frühgeburtenniere auch in dieser Hinsicht eine gewisse Sonderstellung gegenüber der Niere beim anderen und älteren Kinde einnimmt (siehe Protokolle 2, 6, 7, 8).

\section{B. Die Reaktion und die $\mathrm{CO}_{2}$-Regulationsbreite des Blutes bei Intoxikation.}

Ich war imstande, im ganzen bei 20 Intoxikationen das Blut zu untersuchen. In 10 von diesen Fällen im Leben während des toxischen Stadiums; von letzteren starben 5 , bei denen ich dazu noch im Herzblute weitere Bestimmungen vornehmen konnte. Es stellt sich dabei folgendes heraus: viermal wurde schon im Leben eine sichere ,echte“ Acidosis mit vermehrter Wasserstoffionenkonzentration 
des Blutes festgestellt (Protokoll 69, 70, 82, 54) sechsmal war aber trotz schwerer oder leichterer Benommenheit infolge der Intoxikation keine sichere Vermehrung der freien Wasserstoffionen im Blute nachzuweisen (Protokoll 71, 74, 75, 77, 84, 86). In diesen letzten Fällen war das Blut im toxischen Stadium sogar alkalischer als späterhin nach dem Verschwinden der toxischen Erscheinungen, so z. B. im Falle 84, wo der regulierte Wasserstoffexponent während des toxischen Stadiums $P_{H}=7,66$ gegenüber $P_{H}=7,45$ drei Tage später, wo das Kind nicht mehr benommen war, betrug. Ebenfalls im Falle 74; da war die aktuelle Reaktion des Blutes zuerst $\mathrm{P}_{\mathrm{H}} 7,41,4$ Tage später bei leichter Benommenheit des Kindes $P_{H} 7,57$; und nach weiteren 2 Tagen, als das Kind wieder klar war, $P_{H} 7,37$. So war auch im Fall 77 die aktuelle Reaktion des Blutēs bei stark getrübtem Sensorium 6 Tage vor dem Tode $P_{H}=7,64$ gegenüber $P_{H}=6,87$ im Herzblute, das gleich nach dem Tode entnommen wurde. In allen diesen Fällen, in denen das Blutwährend des toxischen Stadiums alkalischer als normal gefunden wurde, war ein typisches tiefes, beschleunigtes toxisches Atmen zu beobachten. Diese eigentümliche unerwartete Erscheinung könnte meiner Ansicht nach wohl als eine Überkompensationserscheinung aufgefaßt werden. Durch das forcierte Atmen ist durch die Lunge nach aller Wahrscheinlichkeit relativ mehr $\mathrm{CO}_{2}$ ausgeschieden worden, als durch die neu entstandenen anderen sauren Stoff wechselprodukte ersetzt werden konnte. Mit Ausnahme von Fall 77, der starb, wurden alle diese Kinder, bei denen im toxischen Stadium eine Überkompensation, d. h. vermehrte Alkaleszenz des Blutes, nachgewiesen werden konnte, wieder hergestellt und geheilt entlassen. Von den 4 Fällen, bei denen im Leben eine ,echte" Acidose festgestellt wurde, kam nur ein einziger Fall (82) zur Heilung. Hier war bei einem bisher ganz gesunden Brustkinde gelegentlich eines akuten, heftigen Durchfalls eine Intoxikation aufgetreten. Auf der Höhe dieses Symptomkomplexes mit deutlich getrübtem Sensorium zeigte das Blut eine starke ,echte“ Blutacidose mit nur $P_{H}=7,09$. Das Kind erholte sich unerwartet rasch. Dieser Fall, der vorläufig einzig dasteht, betraf das nächst jüngste der von mir untersuchten toxischen Säuglinge. Bei ihm war, wie bei den ganz jungen Säuglingen überhaupt, die regulatorische Fähigkeit des Blutes besonders gering, daher die Vermehrung der (H) im Blute; nur der ausschließlichen Frauenmilchernährung haben wir hier wohl den günstigen Ausgang zuzuschreiben.

Bei einem weiteren toxischen Kinde, Fall 70, fand ich in den drei 
letzten Tagen vor dem Tode ebenfalls eine starke „echte“ Blutacidose $P_{H}=7,05$ resp. 7,09, resp. 7,03, im Herzblute 7,00. Dieser Fall zeigt, daß der Tod nicht unmittelbar zu erfolgen braucht, wenn auch die aktuelle Reaktion des Blutes sich beträchtlich nach der sauren Seite hin verschoben hat (siehe auch die Krankenkurve des Falles 70, die bei dem Protokoll liegt).

Die stärkste Säuerung des Blutes im Leben war im Falle 54 zu finden. Da handelt es sich um eine Frühgeburt, die seit Tagen dünne Stühle hatte und benommen war. $P_{\mathrm{H}}=6,54$ des kurz vor dem Tode entnommenen Blutes war also noch viel saurer als in dem vorangehenden Falle.

Wenn auch demnach bezüglich der aktuellen Reaktion des Blutes

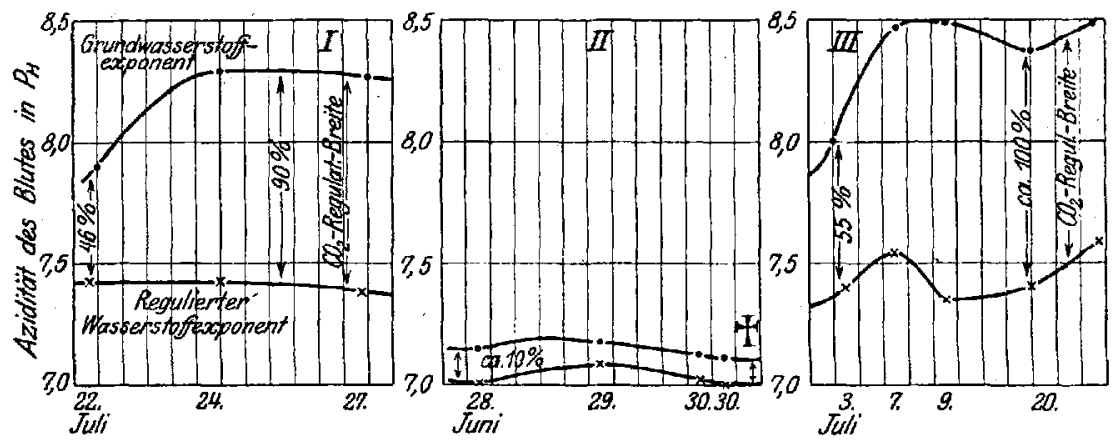

Fig. 22. Die Reaktion und die $\mathrm{CO}_{2}$-Regulationsbreite des Blutes bei Intoxikation.

I und III leichtere Fälle mit Hellung (Protokoll 75 und 74); II: schwerer Fall (70) mit Exitus. Obere Linie: Grund-Wasserstofiexponent; untere Linie: regulierter Wasserstoffexponent.

bei Intoxikation individuelle Unterschiede festzustellen waren, so verhielten sich toxische Kinder bezüglich der $\mathrm{CO}_{2}$-Regulationsbreite prinzipiell gleich. Uberall war eine starke Verschmälerung der $\mathrm{CO}_{2}$-Regulationsbreite nachzuweisen. Nur in seltenen Fällen von echter Blutacidose kam diese Verschmälerung aus den bei der Methode besprochenen Gründen nicht zur Geltung. In den Intoxikationsfällen, die zur Heilung kamen, schwankte die $\mathrm{CO}_{2}$-Regulationsbreite im toxischen Stadium zwischen 46 und $62 \%$, gegenüber $85-113 \%$ in der Rekonvaleszenz; in den Fällen, die mit dem Tode endigten, bewegten sie sich von ea. $8-\mathbf{4 0} \%$. So war sie z. B. im Falle 70 in den drei letzten Tagen vor dem Tode $15 \%$ resp. $9 \%$, resp. $8 \%$ und beim Tode selbst $11 \%$ (Fig. $22 \mathrm{II}$ ).

Mit Ausnahme von einem einzigen Befund (Fall 89) war die Wasserstoffionenkonzentration im unmittelbar nach dem Tode entnommenen 
Herzblute von sowohl toxischen wie nicht toxischen Säuglingen deutlich vermehrt. Bei Kindern, die in den letzten Tagen vor dem Tode toxisch gewesen waren, wurde mit Ausnahme des Falles 89 regelmäßig das am meisten saure Blut gefunden (siehe die vorigen Fälle, außerdem Fall 83, 85, 80, 81, 79, 77, 76 usw. in den Protokollen). Hier schwankte die aktuelle Reaktion zwischen $P_{H} 6,5$ bis ca. 7,0 , denen gegenüber bei Kindern, die aus verschiedenen Gründen mit freiem Sensorium bis vor dem Tode gestorben waren, Werte von $P_{H} 7,20 \ldots-7,40$ (siehe Fall 87,90 u. a.) gefunden wurden. Die regulatorische Fähigkeit des Blutes scheint beim Herannahen des Todes so stark nachzulassen, daß die Konstanz der Reaktion auch schon beim Eintreten des Todes nicht mehr hat aufrechterhalten werden können.

Wenn ich hier schon das Ergebnis dieser Untersuchungsreihe zusammenfassend darlege, so würde es dahin lauten:

Bei Ernährungsstörungen und anderen Krankheiten, in deren Verlauf ein sog. toxischer Symptomenkomplex auftritt, ist in der Mehrzahl der Fälle mit tödlichem Ausgang in den letzten Tagen eine sichere „echte" Blutacidose mit vermehrter Wasserstoffionenkonzentration vorhanden. In Fällen, die in Heilung übergehen, ist eine ,echte" Blutacidose äußerst selten zu finden. Beim Tode ist fast bei jedem Kinde eine echte Blutacidose vorhanden, bei Kindern, die unter dem Bilde der Intoxikation zugrunde gegangen sind, ist die Vermehrung der Wasserstoffionenkonzentration im Blute größer als bei Säuglingen, die an anderen Krankheiten zugrunde gegangen sind. Die $\mathrm{CO}_{2}$-Regulationsbreite ist in jede $\mathrm{m}$ Falle von Intoxikation bedeutend schmäler als normal. In Fällen, die in Heilung übergehen, findet man im Reparationsstadium schon frühzeitig normale Werte wieder. Die Verschmälerung ist auch in diesen Fällen bedeutend geringer als in den Fällen mit letalem Ausgang. Da ist die $\mathrm{CO}_{2}$-Regulationsbreite in den letzten Tagen sehr gering (bis ca. 10\%) und wird noch geringer oder bleibt hier bis zum Eintreffen des Todes (siehe hierbei Fig. 33, S. 365).

\section{C. $0_{2}$-Dissoziationskurve des Blutes bei Intoxikation (Protokolle Fall 76-78, 83-90).}

Durch Bestimmung der $\mathrm{O}_{2}$-Dissoziationskurve bei Intoxikation hoffte ich einen guten Einblick in die tieferen Stoffwechselvorgänge bei derselben zu gewinnen. Die $\mathrm{O}_{2}$-Dissoziationskurve gibt ja neben dem, daß sie ein guter Indicator für den acidotischen Zustand ist, 
auch einen ausgezeichneten Einblick in die oxydativen Funktionen des Blutes. Wir können ja aus dem Verlauf der $\mathrm{O}_{2}$-Dissoziationskurve ohne weiteres schließen, ob das betreffende Blut imstande ist, viel oder wenig $\mathrm{O}_{2}$ mit sich ins Gewebe zu führen, außerdem ob das Blut leicht oder schwer $\mathrm{O}_{2}$ dem Gewebe abgeben kann. Die $\mathrm{O}_{2}$-Dissoziationskurve des Blutes schien mir demnach ein gutes Mittel an die Hand zu geben, die Intensität der viel bestrittenen Oxydationsprozesse bei Intoxikation zu studieren.

Ich habe im ganzen bei acht toxischen Kindern die Dissoziationskurven bestimmt. Bei drei von diesen Kindern außer nach dem Tode im Herzblute, auch schon im Leben in dem durch Sinuspunktion gewonnenen Blute. Außerdem bei zwei weiteren Kindern (Bronchopneumonie und Lues congenita) ${ }^{1}$ ).

Es zeigte sich, daß bei toxischen Kindern die $\mathrm{O}_{2}$-Bindung des Blutes auffallend gering ist, im Vergleich zum Blute des gesunden Menschen. Z. B. im Falle 83 (Kind Müller) war die Abweichung der Dissoziationskurve von der Normalkurve sehr groß. Hier handelte es sich um ein $2^{1} / 2$ Monate altes, künstlich ernährtes, schwer toxisches Kind, das schon seit 4 Tagen schwer benommen war. Die $\mathrm{O}_{2}$-Bindung des Blutes, das einen Tag vor dem Tode entnommen wurde, betrug z. B. bei $35 \mathrm{~mm}$ $\mathrm{Hg} \mathrm{O}_{2}$-Partialdruck nur $50 \%$ gegenüber $92 \%$ beim gesunden Menschen. Dieser auffallend niedrige Verlauf der Dissoziationskurve ist ja in voller Ubereinstimmung mit der Reaktion des Blutes, die eine deutliche Verschiebung nach der sauren Seite hin zeigte. Der Grund-Wasserstoffexponent des Blutes war nur $P_{H}=7,10$, die aktuelle Reaktion $P_{H}=$ 6,96. Die Dissoziationskurve des Herzblutes von demselben Kinde zeigte einen noch niedrigeren Verlauf. $\mathrm{Da}$ war die prozentuelle $\mathrm{O}_{2}$-Sättigung des Blutes bei demselben $\mathrm{O}_{2}$-Partialdruck $(35 \mathrm{~mm} \mathrm{Hg}$ ) nur noch ca. $32 \%$ und die Reaktion des Blutes zeigte auch eine dementsprechende vermehrte Säuerung. Die aktuelle Reaktion war $P_{H}=6,58$ und der Grund-Wasserstoffexponent $P_{H}=6,65$. Die beiden Kurven sind auf Fig. 23, Kurve 1 und 2 dargestellt.

Dieselbe Fig. 23 zeigt zwei weitere Dissoziationskurven I und II, die die Kurven von einem leichteren Falle (Fall 84 Kind Piskol) von

1) Bei Beginn dieser Arbeit habe ich in der Berlin. Physiol. Gesell. im Anschluß an einen anderen Vortrag vorübergehend auf diese Untersuchungen hingewiesen. Der Bericht hierüber (Berlin. Klin. Wochenschrift 1915. 5. 252) ist teilweise unrichtig. Ich betonte damals nur, daß die ganz gewaltigen Abweichungen von der Norm erst kurz vor dem Tode stattfinden. 
Intoxikation darstellen. Es war ein Ammenkind, das 4 Monate lang an der Brust gut gediehen und dann abgesetzt worden war. Nach 1 Monat künstlicher Ernährung (1/2-Milch) akuter Durchfall mit

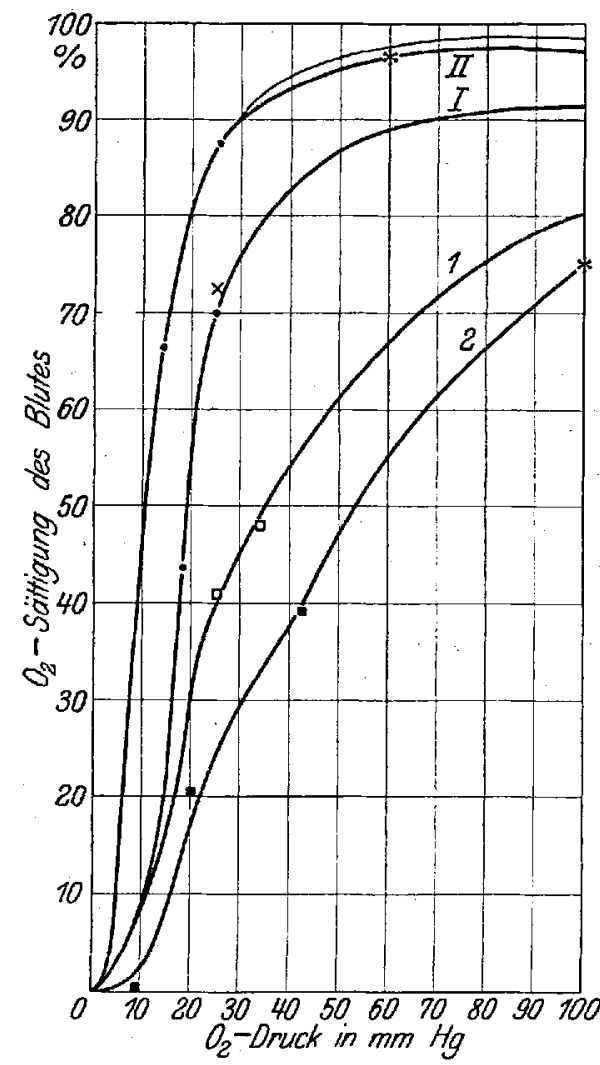

Fig. 23. O,-Dissoziationskurve des Blutes bei Intoxikation. I und II Fall 84, geheilt, I = Kurve während des toxischen Stadiums, II = Kurve in der Rekonvaleazenz. 1 und 2 Fall 8!3 (Kind Miuller) $\dagger$. $1=$ Kurve ca. einen Tag vor dem Tode; $2=$ Kurve nach dem Tode. Dünne Linie $=$ Kurve des gesunden Menschen, $x=$ ein Punkt der Kurve rom Fall 86 Intoxikation, geheilt. $*=$ Punkt aus der Formel $\frac{Y}{100}=\frac{K \cdot x^{n}}{1+K \cdot x^{n}}$ berechnet. demonstriert den Verlauf der Kurve und zeigt dabei, daß die Kurve schọ einen viel höheren Verlauf hat und beinahe mit der Normalkurve zusammenfällt.

Auf derselben Figur ist weiterhin noch ein Punkt mit Zeichen $x$ eingetragen. Dieser ist aus dem Blute eines weiteren leicht toxischen 
Kindes (Fall 86 ) bestimmt. Dieser Fall kam wie der vorige zur Heilung und der bestimmte Punkt zeigt auch, daß seine Dissoziationskurve annähernd mit dem vorangehenden Falle während des toxischen Stadiums übereinstimmt.

Die Kurven auf Fig. 23 stellen gewissermaßen die Verhältnisse für die äußersten Grenzfälle der Intoxikation dar; und zwar können die beiden oberen Linien als typisch für die leichten Fälle von Intoxikation, die beiden unteren Linien als typisch für die allerschwersten Fälle betrachtet werden. Dazwischen liegen die Dissoziationskurven von allen anderen Intoxikationen. Bei letzteren lassen sich schon aus dem klinischen Bilde, der Intensität der Durchfälle und der Dauer des toxischen Stadiums gewisse Schlüsse auf die $\mathrm{O}_{2}$-Bindung des Blutes ziehen.

Besonders interessant waren die Verhältnisse bei einem Fall von Intoxikation beim Brustkinde. Hier handelt es sich um ein 19 Tage altes Kind (Fall 76), das an der Brust dünne Stühle ohne Erbrechen und hohes Fieber bekam. Am zweiten Tage starke Apathie, die am vierten zum tiefsten Komazustand führte, in dem das Kind am gleichen Tage starb. Die Dissoziationskurve des gleich nach dem Tode entnommenen Herzblutes zeigte, trotzdem die Reaktion des Blutes verhältnismäßig starke Verschiebung nach der sauren Seite hin (GrundWasserstoffexponent 6,92, regulierter Wasserstoffexponent 6,85) nachwies, doch nicht so hochgradige Abweichung von dem Verlauf der normalen Kurve wie z. B. bei dem oben besprochenen Fall 83 (Kind Müller). Ob dieser Befund irgendwie im Zusammenhang mit der vorangegangenen Frauenmilchernährung zu bringen ist, oder ob dabei die verhältnismäßig kurze Krankheitsdauer ausschlaggebend gewesen ist, kann ich natürlich auf Grund dieses einen verhältnismäßig seltenen Falles nicht entseheiden. In Anbetracht meiner oben besprochenen Hungerversuche, in denen das Brustmilchkind merkbar weniger Neigung zur Acidose als das künstlich ernährte Kind zeigte, ist aber auch hier anzunehmen, daß die gleiche schützende Rolle der Frauenmilchernährung mildernd auf die acidotischen Veränderungen im Verlaufe der Intoxikation gewirkt hat.

Ich habe die Kurve von diesem Kinde auf Fig. 24 III dargestellt. Auf dieselbe Figur habe ich zum Vergleich Dissoziationskurven von verschiedenen anderen Kindern gebracht. Die unterste Linie IV zeigt die Dissoziationskurve des Herzblutes vom Fall 83, die auch bereits auf Fig. 23 dargestellt worden ist. Linie II stammt vom Herzblute einer kleinen Frühgeburt von $1000 \mathrm{~g}$ (Fall 59 ), die im Alter von 15 Stunden 
an Lebensschwäche starb. Die Linie I, Fall 90 (Maier) bezieht sich auf ein atrophisches Kind, das an chronischer Bronchopneumonie zugrunde ging. Dieses Kind bekam in der letzten Zeit 250-500 g Frauen-

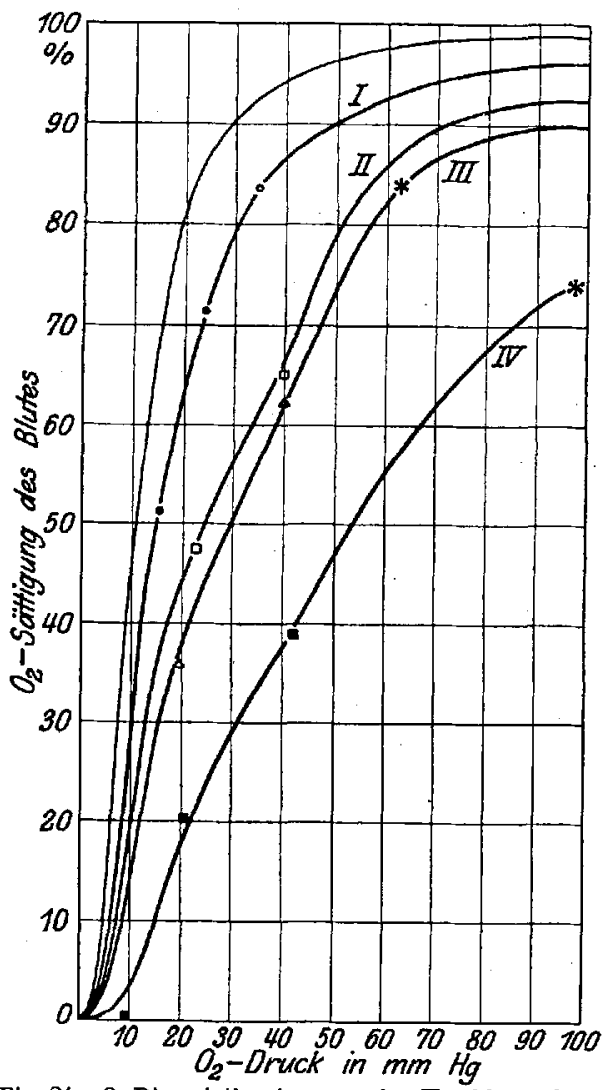

Fig. 24. $\mathrm{O}_{2}$-Dissoziationskurven des Herzblutes bei an verschiedenen Krankheiten verstorbenen Säuglingen. $I=$ Fall 00, $A$ trophie und chronische Broncho . pneumonie; II = Fall 59 , Frühgeburt von $1650 \mathrm{~g}$, 12 std. alt, + ; III = Fall 70 , Intoxikation an der Brust, 19 Tage alt; IV = Fall 83, schwere prolongierte Intoxikation. * = aus der Formel $\frac{Y}{100}=\frac{K \cdot x^{n}}{1+R \cdot x^{n}}$ berechnete Punkte. Dünne Linie $=$ Normalkurve, $\mathrm{CO}_{\mathrm{a}}=0$ bei $38^{\circ}$. Tode bei chronischer Bronchopneumonie, wie oben erwähnt, ausschließlich Frauenmilch bekommen.

Die Fig. 24 zeigt demnach, daß die $\mathrm{O}_{2}$-Bindung des Blutes beim Eintreten des Todes bei diesen Kindern ganz verschieden gewesen ist. Die bei anderen Kindern gefundenen Dissoziationskurven fallen je nach

mileh täglich; das Sensorium war frei, Stühle etwas zerfahren. Das Herzblut zeigte ganz beträchtliche Vermehrung der Wasserstoffionenkonzentration bei der aktuellen Reaktion. Die Vermehrung waraber hauptsächlich auf eine Anhäufung von $\mathrm{CO}_{2}$ zurückzuführen, die $\mathrm{CO}_{2}$-Regulationsbreite war auffallend hoch, $88 \%$, die GrundWasserstoffzahl war dem entsprechend hoch $P_{\dot{H}}=\mathbf{7 , 5 7}$ und infolgedessen wurde bei dem Kinde im Herzblute eine Dissoziationskurve gefunden, deren Verlauf nur verhältnismäßig wenig von dem normalen abwich. Die prozentuelle $\mathrm{O}_{2}$-Sättigung derselben betrug ca. $84 \%$ bei $35 \mathrm{~mm} \mathrm{Hg} \mathrm{O}_{2}$-Partialdruck, gegenüber der entsprechenden Sättigung $93 \%$ normalerweise. Die verhältnismäßig geringe Acidose bei diesem Atrophiker wird wohl auf die ,anti-acidotischen" Eïgenschaften der Frauenmilch zurückzuführen sein, die ich später noch genauer besprechen werde. Das Kind hatte ja wochenlang vor seinem 
den Diagnosen und klinischen Erscheinungen im großen und ganzen in den Rahmen dieser Kategorien, und kann ich diese Kurven als Charakteristicum je für ähnliche Fälle bezeichnen.

Auf Grund alles Obigen kann ich bezüglich der Dissoziationskurve des Blutes folgendes sagen:

Die $\mathrm{O}_{2}$-Bindung des $\mathrm{B}$ lutes ist bei Intoxikation stark herabgesetzt ${ }^{1}$ ); das Blut kann unter gleichen Bedingungen z. B. bei $35 \mathrm{~mm}$ $\mathrm{Hg} \mathrm{O}_{2}$-Partialdruck oft nur den dritten Teil von den normalen Mengen unter gleichen Bedingungen binden. In Fällen von Intoxikation, die in Heilung übergehen, ist die Herabsetzung des $\mathrm{O}_{2}$-Bindungsvermögens während des toxischen Stadiums deutlich vorhanden, beim Klarwerden des Sensoriums kehren aber bald die normalen Verhältnisse zurück. In den Fällen mit letalem Ausgang ist die $\mathrm{O}_{2}$-Bindung beträchtlich niedriger und sinkt sukzessive bis zum Tode herab. Bei anderen parenteralen Erkrankungen (Bronchopneumonia, Lues) ist ebenfalls eine Verminderung in der $\mathrm{O}_{2}$-Bindung im Herzblute nachzuweisen. Die Herabsetzung ist aber bedeutend geringer als bei den Kindern, die unter Intoxikationserscheinungen zugrunde gegangen sind.

Bei einem toxischen Kinde wird demnach bedeutend weniger $\mathrm{O}_{2}$ mit dem Blute ins Gewebe geführt und es ist demnach ganz klar, daß bei Intoxikation die Gewebe unter einem gewissen $\mathrm{O}_{2}$ Mangel leiden müssen, der ja bekanntlich die normalen $0 x y d a-$ tionsvorgänge beeinträchtigt, so daß im Stoffwechsel manche Zwischenstufen entstehen können, darunter Produkte mit saurem Charakter (Acetonkörper usw.), die dann ihrerseits imstande sind, die Acidose selbst zu erzeugen.

In diesem Zusammenhange ist aber auf einen experimentellen Befund von Mathiso ${ }^{2}$ ) bezüglich der $\mathrm{O}_{2}$-Abgabe einer mit Säure versetzten Hämoglobinlösung hinzuweisen. Er hat nämlich gefunden, daß Hämoglobinlösungen, die mit Säure versetzt waren, in relativ viel kürzerer Zeit $\mathrm{O}_{2}$ abgaben, als dieselben Hämoglobinlösungen ohne

1) Auch Howland und Marriott sprachen in "A discussion of acidosis \&e." in New York Academy of Medicine, Section on Pediatrics, 2. XII. 1915, von herabgesetztem Verlauf der Dissoziationskurve bei Intoxikation. Die Arbeit ist meines Wissens bisher noch nicht veröffentlicht worden. Siehe auch S. 281, Anm. 7 dieser Arbeit.

Anmerk. bei der Korrektur: Die Arbeit ist inzwischen erschienen, siehe näheres S. 281, Anm. 8 dieser Arbeit.

2) Mathison, The Influence of Acids upon the Reduction of arterial Blood. Journ. of Physiol. 43, 347. 1911. Siehe auch S. 306 dieser Arbeit. 
Säure unter den gleichen Bedingungen. Ich möchte diesem Befunde eine gewisse Bedeutung bezüglich der Intoxikation zuschreiben, wo eine Blutsäuerung, entweder eine echte oder eine verschleierte, wie oben gezeigt, auftritt. Man könnte sich vorstellen, daß in dieser erleichterten $\mathrm{O}_{2}$-Abgabe des Hämoglobins im sauren Blute ein gewisses Schutzmittel der Natur zu erblicken ist. Bei Intoxikation wird mit dem Blute weniger $\mathrm{O}_{2}$ dem Gewebe zugeführt, was die Entstehung der Acidose befördert, aber gleichzeitig wird von dem Blute verhältnismäßig leichter $\mathrm{O}_{2}$ an das Gewebe abgegeben und dadurch die schädliche Wirkung der verminderten Zufuhr einigermaßen paralysiert resp. kompensiert.

\section{Die Reaktion der Gewebe bei Intoxikation. (Protokolle 62-72. $76-81,85,88$.}

Die Bestimmungen der Reaktion der Gewebe beim hungernden Kaninchen, bei denen ich keine Abweichung gegenüber der Reaktion der Gewebe beim normalen Kaninchen fand, ließen vermuten, daß die sicheren acidotischen Veränderungen während der Intoxikation doch nicht in der Reaktion der Gewebe nachweisbar zur Geltung kämen. Die Untersuchungen belehrten mich aber anders.

Ich habe im ganzen bei zwölf Intoxikationen die Reaktion der Gewebe einschlieBlich des Blutes bestimmt. Die Bestimmungen wurden nach der oben beschriebenen Methode unmittelbar nach dem Tode ausgeführt. In 9 Fällen wurde die Reaktion mehrerer Organe: in der Regel die des Blutes, der Muskulatur, des Herzens, der Leber, Milz und Niere bestimmt, mit Ausnahme von einem Falle sowohl die Reaktion der frischen, wie auch der gekochten Organe. In drei weiteren Fällen wurde neben dem Blute nur die Reaktion der Gehirnsubstanz und der Gehirnflüssigkeit bestimmt.

Im Gegensatz zu dem Befund bei der oben erwähnten Totgeburt, wo die Reaktion der Gewebe hauptsächlich oberhalb der Vergleichslinie $P_{H}=6,5 \cdot \operatorname{lag}$ (siehe Fig. 12, S. 311 dieser Arbeit), befanden sich bei Intoxikation die Werte meistens unterhalb der Vergleichslinie. Zwei Fälle, die eine besondere Phosphatmisching, wie im näohsten Kapitel genauer besprochen wird, erhielten, zeigten Werte, die deutlich oberhalb derselben Linie lagen. Am deutlichsten kommen diese großen Unterschiede im Falle 71 (Kind Baatz, 8 Monate) zur Geltung. Es war ein gut entwickeltes Kind, das an einem heißen Tage plötzlich wässerige Stühle und sehr hohes Fieber bekam. Am dritten Tage wurde es benommen, am vierten Tage zu uns in die Klinik gebracht. Bei der Auf- 
nahme war das Kind tief toxisch, Temperatur bis $41^{\circ}$, kein Erbrechen, Stühle anfangs wässerig, am zweiten Tage gut, am dritten Tage bestand schon Verstopfung. 3 Tage nach der Aufnahme Exitus, nach vorangegangenen Krämpfen und tiefem komatösem Zustande. Die Einzelheiten über den klinischen Verlauf gehen am besten aus der Fig. 25 hervor (siehe außerdem Genaueres im Protokoll 71).

Was die Reaktion der Gewebe betrifft, so sind die Verhältnisse auf Fig. 26 graphisch dargestellt. Wir sehen da zuerst, daß. die Werte für die "Grundreaktion" des Blutes und der Muskulatur etwa im gleichen Gebiete ca. $P_{\mathrm{H}}=7,25$ liegen. Beide sind viel weniger sauer als alle anderen Organe, deren Reaktion nur ca. $P_{H}=6,30$ ist. Demnach liegt hier eine starke Ubersäuerung der Organe im Vergleich zu der des Blutes (auch wenn man die aktuelle Reaktion berücksichtigt) und der Muskulatur vor. Dies ist insofern

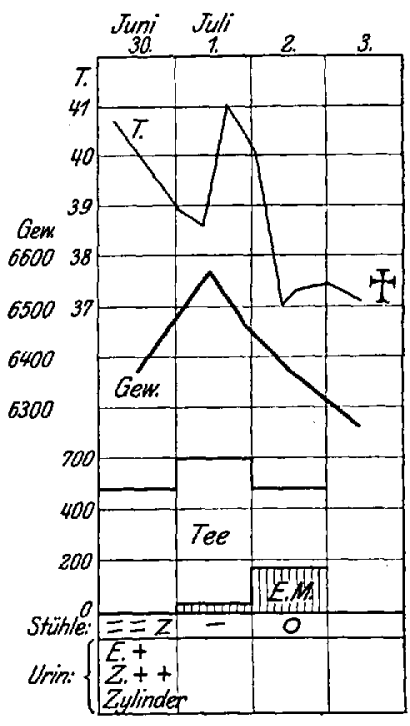

Fig. 25. Fall 71 , Kind Ba tz, 8 Monate alt (Hitze?)-Intoxika tion. von ganz besonderem lnteresse, weil es zeigt, daß nach aller Wahrscheinlichkeit der hauptsächlichste Ort für die Säureproduktion bei

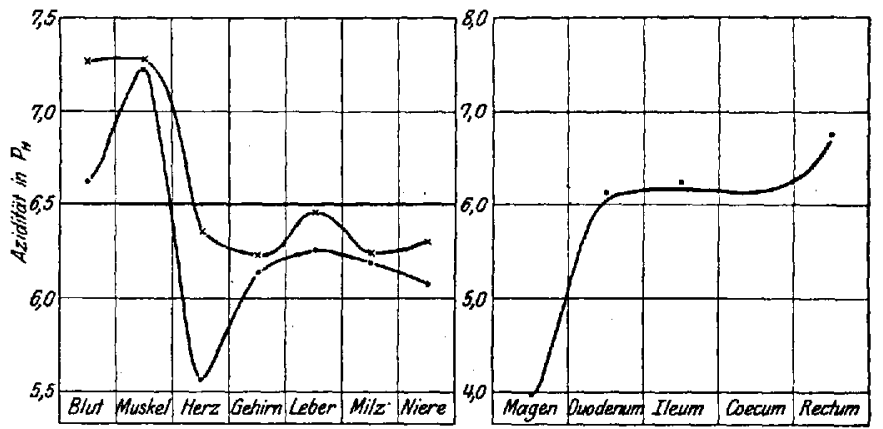

Fig. 26. Die Reaktion des Blutes und der Gewebe bei Iutoxikation. Fall 71, Kind Baatz. Die rechte Seite der Fig. gibt die Reaktion des Magendarminhalts an.

Intoxikation nicht in der Muskulatur, sondern in einem anderen Organ zu suchen ist.

Dieser Befund war aus zwei Gründen befremdend. Erstens weil ich bei hungernden und gesunden Tieren (siehe Fig. 10 und 21) die 
sauerste Reaktion in der Muskulatur gefunden hatte. Dies hatte auch Michaelis und Kramsztyk $\mathbf{k}^{\mathbf{1}}$ ) beim gesunden Tiere konstatiert. Zweitens geht doch die gewöhnliche Ansicht dahin, daß die Muskulatur, in der man während der Arbeit starke Milchsäurebildung festgestellt hatte ${ }^{2}$ ), als der wichtigste Ort für die Säurebildung im Organismus angesehen werden darf.

Der Fall 71 steht nicht vereinzelt da, denn mit Ausnahme von einem einzigen Fall 72 (Intoxikation an der Brust) fand ich bei allen toxischen Kindern die Reaktion der Muskulatur weniger sauer als die der anderen Organe, speziell der Leber und der Milz, wie dies auch aus Fig. 27 hervorgeht.

Auf der Kurve II, der folgenden Fig. 28, ist dasselbe Verhalten deutlich zu erkennen. Ebenfalls besonders schön auf Kurve 31 I und II

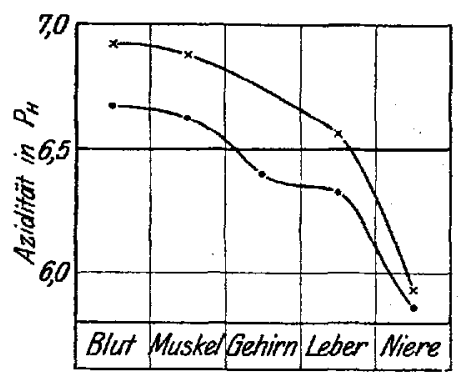

Fig. 27. Die Acidităt des Blutes und der Organe bei Intoxikation. Fall 76 , Kind Sucrow, 19 Tage alt, Intoxikation an der Brust. und auch auf Kurve 30 , die ich aus besonderen Gründen erst in dem nächsten Kapitel bringe.

Auf der Fig. 28 II ist die Reaktion für die Leber und Milz ebenfalls kleiner als die der Körpermuskulatur. Die Reaktion der Herzmuskulatur ist gewiß viel saurer als die der anderen Organe und zeigt eine unerwartet stark saure Reaktion für den frischen Extrakt $P_{\mathbf{H}}=5,28$, eine weniger stark saure für den gekochten Extrakt $P_{H}=6,28$. Die Herzmuskulatur scheint auch in anderen Fällen Neigung zu einer saureren Realktion als die Körpermuskulatur zu zeigen. Dies scheint teils auf vermehrtem $\mathrm{CO}_{2}$-Gehalt des Herzmuskels, teils aber auch auf vermehrtem Gehalt an anderen Säuren zu beruhen, was wohl darauf zurückzuführen ist, daß das Herz im Vergleich zur anderen Körpermuskulatur eine auffallend intensive Arbeit bis zum Tode auszuführen hat. Möglich ist aber auch, daß hier eine besonders intensive postmortale Selbstsäuerung in Frage kommt. Auf derselben Fig. 28 ist das Verhalten der Gewebereaktion auch bei dem oben erwähnten Ausnahmefall 72 auf der Kurve I graphisch dargestellt, dessen

1) Michaelis u. Kramsztyk, Wasserstoffionenkonzentration der Gewebssäfte. Biochem. Zeitschr. 62, 180. 1914. Siehe auch S. 284 dieser Arbeit.

2) Hammarsten, Lehrbuch der physiologischen Chemie. Wiesbaden 1910, S. 562 u. a. Seiten. 
Eigentümlichkeiten ich hierzwischen mit einigen Worten berühren möchte.

Wir sehen da erstens, daß die Reaktion der Körper- und Herzmuskulatur im Gegensatz zu den vorigen Fällen hier viel saurer ist (ca. $P_{H}=6,15$ ) als die Reaktion der Leber, Milz und Niere (ca. $P_{H}=$ 6,60 ), die ja ihrerseits keine besondere Steigerung der Acidität zeigen; liegen doch ihre Werte oberhalb der horizontalen Vergleichslinie. Weiterhin ist in diesem Falle bemerkenswert, daß die Reaktion der Gehirnsubstanz auffallend weniger sauer (ca. $P_{H}=7,10$ ) ist, als alle anderen Organe. Die Erklärungen für die eigentümlichen Abweichungen bei diesem Kinde glaube ich aus der klinischen Vorgeschichte und dem

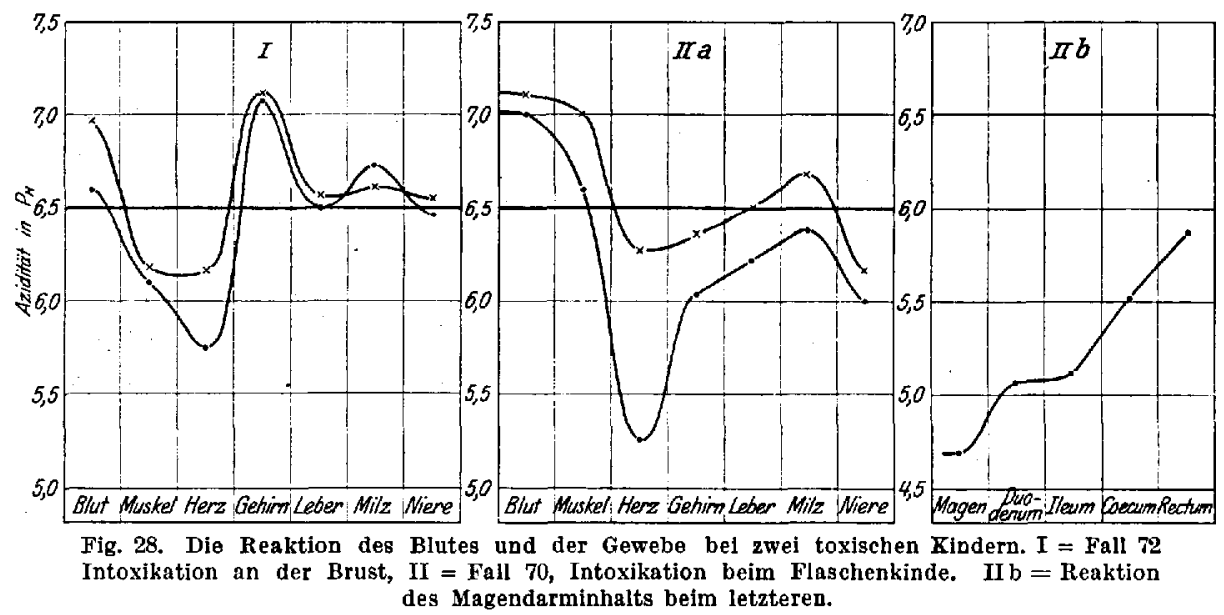

Verlaufe der Intoxikation geben zu können. Es handelte sich hier um ein Brustkind, das plötzlich an der Brust die Nahrung verweigerte, nach ein paar Stunden Krämpfe bekam, schwer benommen wurde und innerhalb ca. 5 Stunden unter hyperpyretischer Temperatur (ca. $40^{\circ}$ ), fortdauernden Krämpfen und Benommenheit zugrunde ging. Die Sektion ergab nur Gehirnödeme und Injektion der Därme als Todesursache. Die stark saure Reaktion der Körpermuskulatur könnte demnach ungezwungenerweise durch die infolge der fortdauernden Krämpfe entstandenen Milch- und anderen organischen Säuren erklärt werden. Und der Umstand, daß die parenchymatösen Organe keine besondere saure Reaktion hatten, hängt wohl in Zusammenhang mit der ganz ungewöhnlich kurzen Dauer der Krankheit. Darauf beruht auch wohl, da $\beta$ die Reaktion der Gehirnmasse nur eine so geringe Verschiebung nach der sauren Richtung hin erlitten hatte. 
Was übrigens die Reaktion der Gehirnmasse und der Gehirn- resp. Lumbalflüssigkeit betrifft, so zeigte sie ebenfalls in der Mehrzahl der anderen Intoxikationen eine geringere Acidität als die der anderen Organe. Dies hat seine Erklärung wohl darin, daß erstens der selbständige Stoffwechsel im Zentralnervensystem sowieso verhältnismäßig gering ist und daß zweitens der Säfteaustausch vom Blute in die Gehirnsubstanz selbst, resp. in die Cerebrospinalflüssigkeit und umgekehrt, der schon normalerweise sehr langsam vor sich geht, in pathologischen Zuständen besonders daniederliegt, wie das u. a. aus Usenersis) Untersuchungen hervorgeht. Ich begnüge mich an dieser Stelle mit dem Hinweis auf diesen Befund und werde später an der Hand einer Spezialtabelle auf diese Frage genauer eingehen.

Es könnte nun der Einwand gemacht werden, daß die stark saure Reaktion der parenchymatösen Organe insbesondere der Leber im Vergleich zur Reaktion des Blutes und der Muskulatur einfach auf besonders lebhafte postmortale Säuerung zurückzuführen ist. $\mathrm{Da}$ dies nicht zutreffen kann, bestätigt schon gewissermaßen der Tierversuch, wo wie oben angegeben, die stärkste postmortale Säuerung in der Muskulatur zu finden war. Und meine Untersuchungen, in denen ich gleichzeitig mit der Leberreaktion auch die Reaktion der Galle bestimmte, sprechen entschieden dagegen. Ich fand nämlich, daß die Reaktion der Galle meistens genau die gleiche war, wie die der Leber; so z. B. im

$$
\begin{aligned}
& \text { Fall } 70 \text { Leber, frisch . . . } \mathrm{P}_{\mathbf{H}} 6,21 \\
& \text { Galle . . . . . , } 6,21 \\
& \text { Fall } 71 \text { Leber, frisch . . . „, 6,36 } \\
& \text { Galle . . . . . . . ,6,14 } \\
& \text { Fall } 77 \text { Leber, frisch . . . , 6,15 } \\
& \text { Galle . . . . . . , 6,04 usw. }
\end{aligned}
$$

Wäre nun die stark saure Reaktion der Leber auf eine postmortale Säuerung zurückzuführen, so müßte die Galle, die ja nur eine lebende Leberzelle sezernieren kann, bedeutend alkalischer sein. Wenn sie nun das nicht ist, sondern dieselbe Reaktion besitzt wie die Leber selbst, so muß man ja annehmen, daß die Reaktion der Leber im Leben schon annähernd so sauer gewesen ist. Die Leber produziert demnach bei Intoxikation cine Galle, die eine auffallend saure Reaktion hat, im Gegen-

1) Usener, Über die Verteilungs- und Ausscheidungsverhältnisse des Urotropin im menschlichen Körper und deren Verwendung zur Differentialdiagnose des Hydrocephalus. Zeitschr. f. Kinderheilk. 8, 111. 1913. 
satz zu der deutlich alkalischen oder strenggenommen neutralen Galle, die eine normale Leber produziert.

Ich bin nach alledem geneigt, diesem Befunde, daß die Leber eine saure Galle bei Intoxikation a usgeschieden hat und daB das Leberparenchym selbst eine stark saure Reaktion beim Eintreten des Todes besitzt, eine prinzipielle Bedeutung beizumessen. Die Leber scheint demnach bei der Säurebildung bei Intoxikation eine große Rolle zu spielen und es scheint beinahe, als ob die Úbersäuerung des Organismus, d. h. die Entstehung der Acidose, in erster Linie auf die Leber als größtes parenchymatöses Organ zurückzuführen ist. Diese Anschauung findet eine gewisse Stütze in den Untersuchungen von Embden und Kalberlah ${ }^{1}$ ), die im Tierversuche gezeigt haben, daß die Fähigkeit, Acetonkörper bei künstlicher Durchblutung zu bilden, von untersuchten Körperorganen nur ausschließlich der Leber zuzuschreiben ist.

Ob die Milz bei der Säurebildung irgendwelche Rolle spielt, weiß man nicht. Die mit der Leberreaktion analog sauren Werte könnte man aber dafür sprechen lassen.

Was die Reaktion der Niere betrifft, so war sie auch oft stark sauer. Ich kann aber diesem Befunde keine besondere Bedeutung beimessen, denn die Niere ist ja sowieso das Sammel- und Ausscheidungsorgan für die sauren Produkte, die natürlich die Reaktion des Nierenparenchyms in erster Linie beeinflussen. So fand ich ja auch oft die Reaktion der Niere annähernd gleich der Reaktion des kurz vor dem Tode gelassenen oder in der Blase gefundenen Urins (siehe z. B. Fall 70, Urin $P_{\mathrm{H}}=5,62$, Niere $\mathrm{P}_{\mathrm{H}}=6,00$ ).

Zusammenfassend läßt sich das Ergebnis dieser Untersuchungsreihe in folgenden Sätzen darlegen: Die Organe von toxischen Kindern zeigen eine deutliche Vermehrung der Acidität im Gegensatz zu den Organen einer Totgeburt und den Organen von gesunden Tieren. Die Reaktion der parenchymatösen Organe ist in der Mehrzahl der Fälle sauerer als die Reaktion der Muskulatur und des Gehirns. Weil die Galle dieselbe Reaktion wie die Leber zeigt, so wird die saure Reaktion der Leber als schon zum Teil im Leben existierend betrachtet. Und der Leber wird hiermit eine große Rolle bei der Entstehung der sauren Stoffwechselprodukte bei Intoxikation zugeschrieben. Die Reaktion des Zentralnerven-

1) Embden u. Kalberlah, Ưber Acetonbildung in der Leber. Beiträge z. chem. Physiol. u. Pathol. 8, 121. 1906. 
systems ist meistens weniger sauer als die anderer Organe. Dies wird mit dem langsamen Säfteaustausch zwischen dem Blute und dem Zentralnervensystem und mit dem geringen Stoffwechsel in diesem in Zusammenhang gebracht.

\section{E. Die Reaktion des Magen- und Darminhalts bei Intoxikation. (Protokolle Fall 69, 70, 71, 76, 78, 79.)}

Um einen Einblick in die Aciditätsverhältnisse des Magen- und Darminhalts bei Intoxikation zu erhalten, habe ich in mehreren Fällen gleich im Anschluß an die Bestimmung der Organreaktion auch die Reaktion des Magendarminhalts bestimmt. Ich habe das dabei benutzte Verfahren bereits bei der Besprechung der Methode in Kürze erwähnt (S. 285 dieser Arbeit). Es zeigte sich in diesen Untersuchungen erstens : daß die Acidität des Magendarminhalts ebenso wie die Acidität des Stuhles in hohem Grade von der vorangehenden Nahrung abhängig ist; zweitens, daß die Acidität des Magendarminhaltes sukzessive abnimmt: der Mageninhalt ist immer am sauersten, meistens ca. $P_{H}=4,0-5,0$, der Inhalt des Duodenums viel weniger sauer und dann der Reihe nach Ileum-Coecum-Rectum-Inhalt. Die Aciditätskurve in $\mathbf{P}_{\mathbf{H}}$ ausgedrückt, bildet hierdurch eine aufsteigende Linie, wenn man den Inhalt vom Magen nach dem Rectum hin verfolgt (siehe Fig. 26, 28, 30, 31). Die Endwerte für den Inhalt im Rectum sind bei vorangehender Frauenmilchernährung etwa wie die Werte für die Stuhlacidität bei Frauenmilchernährung bis ca. $P_{H}=5,5$. Finden aber Blutungen oder sonst besonders reichliche Exsudationen im Magen oder im Darm statt, so kann die Reaktion auch bei Frauenmilchernährung interessanterweise leicht alkalisch werden, wie dies z. B. Fall 76 beweist, in dem die Werte ziemlich gleichmäßig, sowohl für den Darm- wie für den Mageninhalt innerhalb $\mathrm{P}_{\mathrm{H}}=6,80-7,50$ schwankten.

Dies alles zeigt, daß die Acidität des Stuhls von allzu vielen Faktoren abhängig ist, die man kennen müßte, ehe man irgendwelche Schlüsse über die Acidität des Magen- und Darminhaltes bei Intoxikation ziehen könnte. Auf Grund der obigen Untersuchungen steht das eine nur fest, daß im Darm selbst keine besonders hohen Aciditätswerte zu finden waren, wie man möglicherweise hätte erwarten können. Die regelmäBige Abnahme der Acidität, je mehr man im Darme nach abwärts geht, spricht, wie ioh in allen meinen Fällen konstatieren konnte, jedenfalls deutlich dagegen, daß irgendwo im Dünndarm größere Säuremengen entstehen, die die dyspeptischen Erscheinungen hervorrufen 
sollten [Salge'), Tobler und Bessa $\left.\mathrm{u}^{2}\right)$ ]. Würde im Dünndarme irgendwelche beträchtlichere Säureproduktion [durch Bakterien, Salge ${ }^{\mathbf{1}}$ )] stattfinden, so müßte doch in diesem Abschnitt eine größere Acidität des Darminhaltes konstatierbar sein, was ich aber nicht habe bestätigen können. Statt Säuerung des Darminhaltes findet man im Darmkanal eine stetige Abnahme der Acidität, die wohl nur so zu erklären ist, da $B$ in der ganzen Länge des Darmes je nach Umständen mehr oder minder (letzteres bei Intoxikation wahrscheinlich) alkalische Darmsekrete ausgeschieden werden, die die Acidität der Nahrung allmählich herabsetzen. Außerdem spielen hier auch Resorptionsvorgänge mit.

Neben der aktuellen Reaktion habe ich gelegentlich bei dem Fall 69 auch die Reaktion des gekochten, von allen flüchtigen Bestandteilen befreiten Darminhalts bestimmt. Auf diese Weise wollte ich mir einen kleinen Begriff machen, in welchem Maße die flüchtigen, freien Fettsäuren und $\mathrm{CO}_{2}$ bei der Reaktion des Dárminhaltes beteiligt sind. Es zeigte sich, daß die Alkalescenz des Stuhles nur in ganz mäßigem Grade zunimmt, wenn man die flüchtigen Säurebestandteile verjagt hat. Im Duodenum stieg $\mathrm{P}_{\mathrm{H}}$ von ca. 7,20 auf ca. 7,90, im Ileum von ca. 7,40 bis ca. 8,30 zurück. Genauere Untersuchungen in dieser Richtung habe ich nicht unternommen, es lag dies ja außerhalb meines Themas. Der einzige Fall würde nur dafür sprechen, daß der Anteil der flüchtigen Säuren bei der Reaktion des Darminhaltes nicht besonders hoch $\mathrm{zu}$ bemessen ist.

\section{Therapeutisches zur Bekämpfung der Acidose bei Intoxikation.}

$\mathrm{DaB}$ die Intoxikation einen gewissen acidotischen Zustand in sich schließt, geht zur Genüge aus dem Vorangegangenen hervor. Nun ist die Intoxikation meistens mit einem absoluten oder relativen Hungerzustand verbunden, und dieser begünstigt ja schon seinerseits die Entstehung der Acidose bei einem gesunden Organismus. Nun wäre der erste Gedanke, im Falle man der Acidose eine schädliche Rolle bei der Intoxikation zuschreibt, der, daß man den Kindern reichlich Nahrung zuführt. Die Nahrungsaufnahme und -resorption ist aber schon wegen des Zustandes des Kindes, teils wegen der Appetitlosigkeit, teils wegen des heftigen Durchfalls im akuten Stadium äußerst gering. Und in den

1) Salge, Der akute Dünndarmkatarrh des Säuglings. Leipzig 1906.

2) Tobler u. Bessau, Allgemeine pathologische Physiologie der Ernährung und des Stoffwechsels im Kindesalter. Wiesbaclen 1914. 
Fällen, wo eine Nahrungsaufnahme von seiten des Kindes möglich ist, geben wir wiederum dem Kinde nur kleine Mengen, weil durch die Empirie in der Klinik nachgewiesen wurde, daß die Entgiftung der toxischen Kinder rascher, wie das besonders von Finkelstein ${ }^{1}$ ) hervorgehoben' worden ist, bei stark beschränkter Nahrungszufuhr eintritt. Es könnte demnach scheinen, daß die Acidose etwas ganz Nebensächliches und Harmloses bei Intoxikation wäre, und doch ist dem wohl nicht so.

Jegliche Verschiebung in der wahren Reaktion der Gewebe bringt unbedingt eine große Beeinträchtigung der Gewebsfunktionen mit sich. Durch die experimentelle Forschung wissen wir, daß z. B. alle enzymatischen Prozesse ebenso wie alle anderen chemischen Leistungen überlebender Organe in hohem Maße von der wahren Reaktion der Durchströmungsflüssigkeit a.bhängig sind. Rona und Wilenko ${ }^{2}$ ) haben zum erstenmal in ihren Untersuchungen über die Glykolyse am überlebenden Kaninchenherzen einwandfrei nachgewiesen, daß schon ganz geringe Verschiebungen nach der sauren Seite hin in der Reaktion der Durchströmungsflüssigkeit eine starke Herabsetzung des Zuckerverbrauchs daselbst hervorrufen.

$\mathrm{Da} B$ ähnliche Abhängigkeitsverhältnisse zwischen der Reaktion der Gewebe und den Funktionen derselben auch beim Säugling und auch im toxischen Stadium existieren, unterliegt keinem Zweifel. Dieser Gedankengang hat mich zu den folgenden therapeutischen Versuchen zur Bekämpfung der Acidose bei der Intoxikation veranlaßt.

Gewöhnlich reicht man heutzutage den toxischen Kindern neben Tee auch Ringerlösung zu dem Zweck, die Exsiccation zu verhindern. Ringerlösung erfüllt auch in dieser Hinsicht ihre Aufgabe gut, versagt aber, wenn man von ihr eine Beeinflussung der Reaktion des Körpers erlangen will. Als Gemisch von neutralen Salzen besitzt sie keine reaktionsregulatorische Fähigkeit, solche können nur sog. „Puffermischungen" ausüben, Mischungen, die gewöhnlich aus einer Säure und ihren Natriumsalzen bestehen, deren Mengenverhältnis in der Mischung für die Reaktion ausschlaggebend ist. Eine Lösung, die die Acidose bekämpfen sollte, mußte so beschaffen sein, daß ihre Reaktion etwa der des normalen Blutes gleich und daß sie imstande ist, diese Reaktion aufrechtzuerhalten, trotz gegenteiliger Wirkung der sauren

1) Finkelstein, loc. cit., S. 278 dieser Arbeit.

2) Rona u. Wilenko, Beiträge zur Frage der Glykolyse. IV. Biochem. Zeitschr. 62, 1. 1914 . 
Blutbestandteile. Aus physiologisch-chemischen Gründen konnten hier nur Bicarbonatlösungen und Phosphatmischungen in Frage kommen. Bicarbonat zusammen mit $\mathrm{NaCl}$ hatten schon $\mathrm{Heim}$ und $\left.\mathrm{John}^{1}\right)^{2}$ ) bei Intoxikation als Mittel gegen Exsiccation empfohlen. Bicarbonatmischungen haben aber viel Nachteiliges gegen sich. Erstens wird bei der forcierten Atmung bei Exsiccation die $\mathrm{CO}_{2}$ bald so weit ausgetrieben, daß die Mischung ihre Reaktion nicht mehr aufrechterhalten kann. Es bleibt hier durch die Abgabe der $\mathrm{CO}_{2}$ durch die Lungen $\mathrm{Na}$ frei, das Anionen vor allem $\mathrm{Cl}$, aus dem Gewebe bindet und auf diese Weise starke Wasserretention [100-600 g Zunahme in den ersten 24 Stunden bei Säuglingen bei Heim und $\left.\operatorname{Joh}^{1}\right)$ ] hervorruft und zu Odemen führt, die ja sehr oft bei Na-Bicarbonat-Medikation auftreten und deren Bedingungen von $\mathrm{Wyss}^{3}$ ) genauer studiert und erklärt worden sind. Es bleiben demnach nur Phosphatgemische übrig.

Nach vielem Hin- und Herprobieren bin ich zuletzt zu einer Mischung gekommen, die genau die Reaktion des normalen Blutes zeigt, und die bei subcutaner Zufuhr in kurzer Zeit eine Urinreaktion hervorruft, die annähernd mit der der eingeführten Mischung übereinstimmt. Die Zusammensetzung der Mischung war folgende:

$\frac{1 / 3 \text { prim. Natriumphosphat }}{1 / 3 \text { sekund. Natriumphosphat }}=\frac{\left.1 \text { Teil }{ }^{4}\right)}{8 \text { Teile }}=15 \mathrm{ccm}$
Natriumchlorid . . . . . . . . . . $5 \mathrm{~g} \mathrm{~g}$
Kaliumchlorid . . . . . . . . . . . $0,1 \mathrm{~g}$
Destill. Wasser . . . . . . . . bis $1000 \mathrm{ccm}$

Dieses Gemisch habe ich im ganzen 18 Kindern gereicht. 16 von diesen litten an verschiedenen Erkrankungen, in 4 Fällen handelte es sich um toxische Kinder. Bei 8 Kindern trat ein mehr oder weniger hohes vorübergehendes Fieber im Anschluß an die Injektion auf. $\mathrm{Ob}$ das Fieber bei dieser Mischung auf die Salzbestandteile zurückzuführen

1) Heim u. John, Über die interne Anwendung von Salzlösungen bei Behandlung der akuten Ernährungsstörungen im Säuglingsalter. Monatsschr. f. Kinderheilk. 6, 561. 1907.

2) Heim u. John, Die Behandlung der Exsiccation mit Salzlösungen. Jahrb. f. Kinderheilk. ro, 96. 1909.

3) v. Wyss, Über Ödeme durch Natrium bicarbonicum. Deutsches Archiv f. klin. Med. 111, 93. 1913.

4) Nach Michaelis wird 1. ,1/3 mol. prim. Natriumphosphat" folgenderweise hergestellt: $100 \mathrm{ccm}$,molare“ oder "dreifach normale" Phosphorsäure (Kahlbaum) $+100 \mathrm{ccm} \mathrm{n-NaOH}+100 \mathrm{ccm} \mathrm{H}_{2} \mathrm{O}$. 2. "1/3 mol. sek. Natriumphosphat": $100 \mathrm{ccm}$ molare Phosphorsäure $+200 \mathrm{~cm} \mathrm{NaOH}$. 
ist, etwa wie Meyer') u. a. das Kochsalzfieber erklären, oder ob die Ursache in irgendwelchen anderen Bestandteilen (bakteriellen oder anderen unbekannten chemischen Giften) zu suchen ist, wie es bei Kochsalzfieber. Samelsohn'); Bendix und Bergmann ${ }^{3}$ ) und Aron ${ }^{4}$ ) behaupten, vermag ich nicht zu entscheiden. Jedenfalls geschah die Resorption in ähnlicher Weise wie sie bei subcutanen Kochsalz- und Ringerlösungen vor sich geht, ohne daß lokale Schädigungen nachzuweisen gewesen wären.

Ich bespreche hier unten in Kürze nur die 4 Fälle von Intoxikation, bei denen ich die Mischung angewandt habe. Zwei von den Kindern

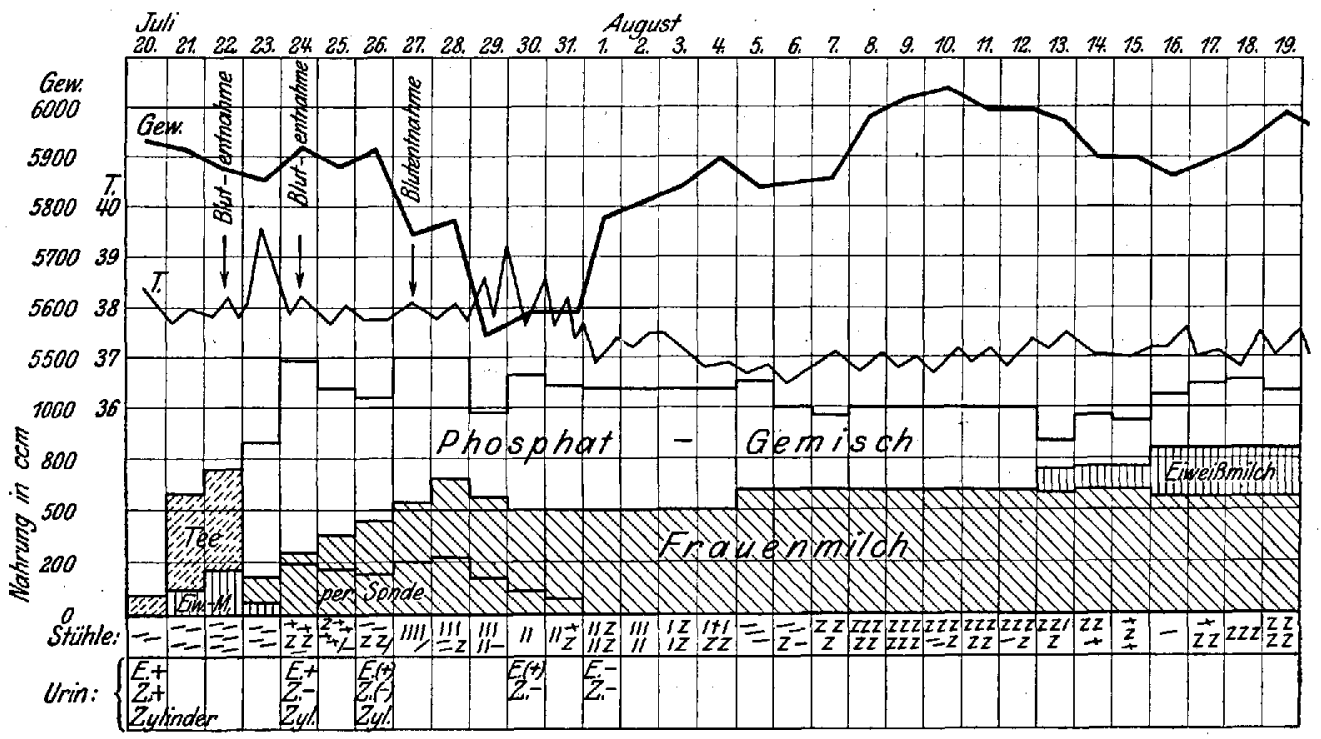

Fig. 29. Krankenkurve Fall 75 (Kind S.) ,Phosphat"kind.

starben schon einen Tag nach der Aufnahme in die Klinik, zwei blieben am Leben und bekamen die Mischung längere Zeit. Die Befunde bei einem von den letzteren lasse ich hier folgen:

1) L. F. Meyer, Experimentelle Untersuchungen zum alimentären Fieber. Deutsche med. Wochenschr. 35, 194. 1909.

2) Samelson, Über das sogenannte Kochsalzfieber. Monatsschr. f. Kinder. heilk. 11, 125. 1912 .

3) Bendix u. Bergmann, Über das sogenannte Kochsalzfieber. Monatssehr. f. Kinderheilk. 11, 387. 1912.

4) Aron, Beobachtungen über die temperatursteigernde Wirkung subcutaner Salzinfusionen beim Säugling. Verhandl d. Gesellsch. f. Kinderheilk., Wien 1913, S. 21. 
Es handelt sich um ein 4 Monate altes, gut entwiékeltes Kind, das im Verlaufe von akutem Durchfall toxisch geworden war. Das Kind wurde am 20. Juli in leicht toxischem Zustande eingeliefert, trank leidlich und bekam, wie aus der beigefügten Kurve genauer zu ersehen, in den ersten Tagen geringe Mengen Eiweißmilch und Tee. Am 22. Juli wurde eine Blutentnahme gemacht und dabei eine auffallend schmale $\mathrm{CO}_{2}$-Regulationsbreite (nur $46 \%$ ) festgestellt. Das Kind war dann leicht toxisch. Vom 23. Juli früh ab erhielt es dann neben Frauenmilch größere Mengen Phosphatgemisch, teils aus der Flasche, teils mit Sonde. Am 24. Juli, wo das Kind noch deutlich toxisch war, nochmalige Blutuntersuchung, die schon eine auffallend hohe $\mathrm{CO}_{2}$-Regulationsbreite $(85 \%)$ zeigte.

Bei der dritten Blutentnahme am 27. Juli war noch eine leichte Apathie vorhanden, trotzdem war die Regulationsbreite auffallend hoch (90\%): Gleichzeitig war eine Abnahme der Urinacidität zu beobachten. Am 23. Juli, ehe das Kind Phosphatgemisch bekommen hatte, zeigte der Urin $P_{\mathrm{H}}=5,61$, am Mittag schon $P_{\mathrm{H}}=6,46$, um 2 Uhr nachmittags $P_{H}=7,15$ und an den folgenden Tagen $P_{H}=7,0$ bis 7,30. Nun wurde aber gleichzeitig vom 23. Juli ab neben Phosphatgemisch auch Frauenmilch gegeben, die im gleichen Sinne wie Phosphatgemisch normalerweise wirkt, so da $\beta$ hier zwei Faktoren vorhanden gewesen sind, was verhindert, die Abnahme der Urinacidität allein dem Phosphatgemisch zuzuschreiben. Jedenfalls habe ich bei einem toxischen Kinde bei Frauenmilch allein so hohe $\mathbf{P}_{\mathrm{H}}$-Werte nie gefunden. Bei dem zweiten Fall von Intoxikation, der zur Heilung kam, verhielt sich die Urinacidität wie hier. Da wurde auch neben Phosphatgemisch Frauenmilch gegeben. Bei Säuglingen, die Phosphatgemisch und irgendwelche Kuhmilchmischung (Eiweißmilch) während des toxischen $\mathrm{Zu}$ standes bekommen hätten und die imstande gewesen wären, die Wirkung des alleinigen Phosphatgemisches auf die Urinacidität bei Intoxikation besser klarzulegen, habe ich keine Versuche anstellen können, weil alle toxischen Kinder Frauenmilch bekamen.

Schöner und einwandfreier geht die Wirkung des Phosphatgemisches bei zwei Säuglingen hervor, die in den ersten 24 Stunden nach der Aufnahme an Intoxikation zugrunde gegangen sind. Da zeigte sich bei der Bestimmung der Organreaktion die eminente Wirkung des Phosphatgemisches.

Im Falle 69 (Kind Fischer), wo es sich um ein schwer toxisches Kind handelte, injizierte ich um 1 Uhr mittags $120 \mathrm{ccm}$ obigen Phosphatgemisches, das langsam noch resorbiert wurde. Nach ca. 1 Stunde trat 
dann Exitus ein. Die Reaktion aller Organe und des Blutes wurde gleich nach dem Tode bestimmt. Dabei stellte sich heraus, daß die Reaktion der Organe viel weniger sauer war als bei allen oben besprochenen Intoxikationen.

Wir sehen, daß die Reaktionswerte, mit Ausnahme der für die Niere, alle oberhalb der Vergleichslinie $\mathbf{P}_{\mathbf{H}}=6,5$ liegen. Dies im Gegensatz zu den oben besprochenen Intoxikationen, bei denen sie meistens unterhalb derselben Linie lagen, besonders die der parenchymatösen Organe. Interessant waren weiterhin die auffallend hohen $P_{H}$-Werte im Darminhalt. Die Reaktion im unteren Abschnitt des Darmes war ja auffallenderweise alkalisch. Diese alkalische Reaktion beruhte, wie genauer
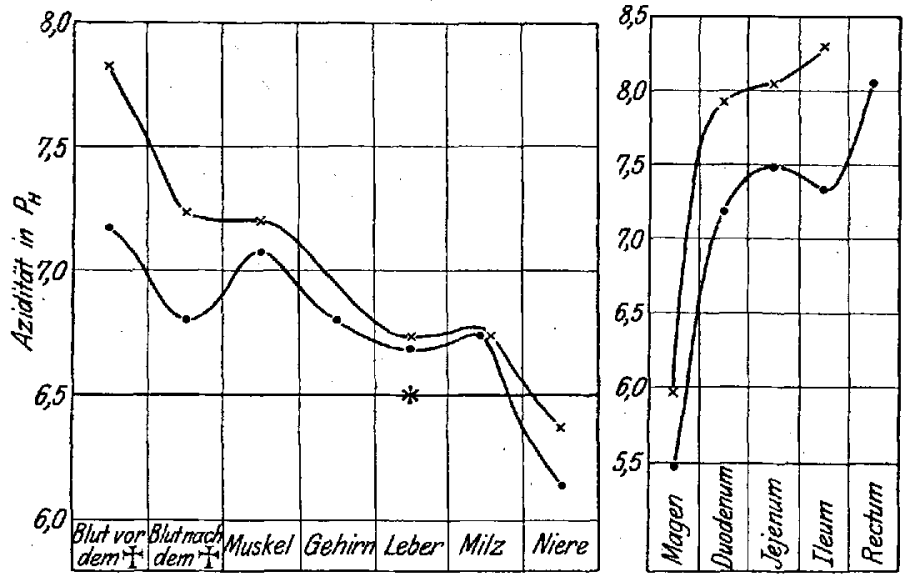

Fig. 30. Reaktion deg Blutes und der Organe bei Intoxikation. „Phosphatkind* Fall 69 (Fischer). Die rechte selte der Kurve zeigt die Reaktion des Magendarminhalts an: untere Linie die des frigchen, obere die des gekochten Inhalts; $*=\mathbf{P}_{\mathrm{H}}$ der Galle.

aus dem Protokoll ersichtlich, auf reichlicher Exsudation des Blutes und der Darmsekrete daselbst, die hier ihrerseits vielleicht dank der Phosphatwirkung keine stärkere Säuerung haben annehmen können.

In Fall 78 waren die Verhältnisse analog zu dem vorangegangenen Fall 69. Hier handelt es sich ebenfalls um ein schwer toxisches Kind, bei dem innerhalb 24 Stunden zweimalige Injektion von je $150 \mathrm{~g}$ Phosphatgemisch vorgenommen wurde. Das Phosphatgemisch resorbierte langsam, aber beim Tode, drei Stunden nach der letzten Injektion, war an der Injektionsstelle keine besondere Schwellung zu beobachten. Die Reaktionswerte für die Organe lagen auch in diesem Falle oberhalb der Vergleichslinie (Pankreas und Niere ausgenommen) und was besonders interessant: die Galle zeigte eine alkalische Reaktion, wie ich 
sie früher nie gefunden hatte. Die Reaktion des Darminhalts verhielt sich genau wie in dem vorigen Falle. Was die Konsistenz und weitere Beschaffenheit desselben betrifft, so ist darüber auch dasselbe zu sagen.

Diese letzten Versuche sind geeignet zu zeigen, daß subcutan in den Körper eingeführtes Phosphatgemisch imstande ist, mit Erfolg die starke Neigung zur Säuerung der Gewebe in toxischem Zustande' zu bekämpfen:

Es wäre recht interessant gewesen, die Einwirkung des Phosphatgemisches auf die $\mathrm{CO}_{2}$-Regulationsbreite und die $\mathrm{O}_{2}$-Dissoziationskurve

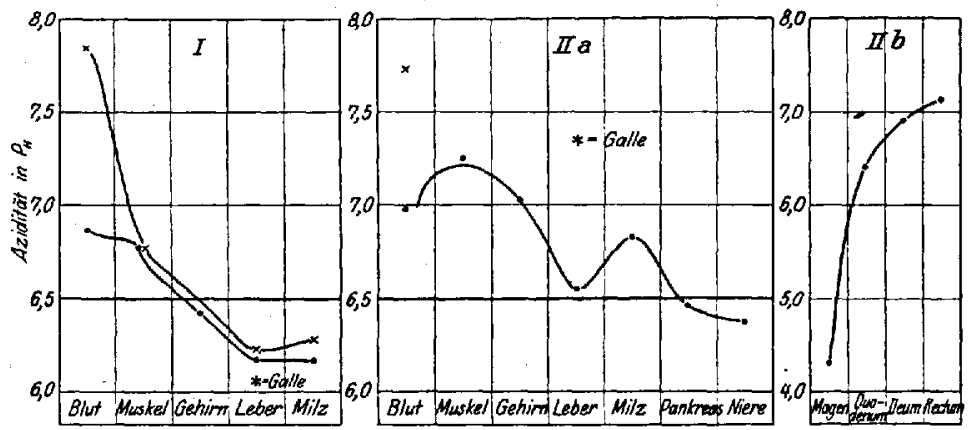

Fig. 31. Reaktion des Blutes und der Gewebe bei Intoxikation. I = toxisches Kind ohne Phosphatgemisch, Fall 77 (Cornet): untere Linie Reaktion der frischen Gewebe, obere Linie die der gekochten Gewebe. II = Phosphatkind, Fall 78 (Kreutzer): a) Reaktion der frischen Organe; b) die des Magendarminhaltes.

zu studieren, um die feinere Beeinflussung des Gemisches auf die reaktionsregulatorischen und oxydativen Fähigkeiten des Blutes kennenzulernen. Dies sind aber Aufgaben, die der Zukunft vorbehalten bleiben. Wenn auch diese therapeutischen Versuche nicht direkt zu meinem Thema gehören, das ja nur das Wesen der Acidose beim Säugling beleuchten soll, so habe ich diese wenigen und unvollständigen Versuche hier angeführt in der Hoffnung, daß diese anderenorts Anregung zur weiteren Forschung auf dieser wenigstens theoretisch gut begründeten Basis geben mögen.

VIII. Besprechung der Ergebnisse mit Berücksichtigung der Klinik.

Hiermit sind meine Untersuchungen abgeschlossen. Es geht aus denselben in der Kürze zunächst der wichtige Befund hervor, da $B$ der menschliche Organismus sich bei der Geburt und in den ersten Lebenstagen physiologisch in einem acidotischen Zustande befindet. Die „Acidose"-Zeichen verschwinden dann, können aber leicht durch einen 
Hungerzustand, beim jungen Säugling in merkbarer Intensität, künstlich hervorgerufen werden und treten bei den sog. Intoxikationszuständen in besonders starker Form hervor.

Diese Tatsachen sprechen dafür, daß die drei Zustände unbedingt in näheren Beziehungen zueinander stehen müssen und es drängt sich die Frage auf: worin bestehen dieselben? Und weiterhin, welche Rolle spielt die Acidose bei Intoxikation? Ist sie hierbei nur ein gelegentliches Symptom oder steht die Entstehung des toxischen Krankheitskomplexes in ätiologischem Zusammenhang mit der Acidose?

Ich will zunächst über die erste Frage einige Worte sagen: Die acidotischen Merkmale des Neugeborenenorganismus, die ich kurzweg als die ,acidotische Konstitution" des Neugeborenenorganis$\mathrm{m} u \mathrm{~s}$ bezeichne, hängen sicherlich in irgendeiner Weise mit dem Graviditätszustand zusammen, der ja, wie oben erwähnt, nachweislich ein acidotischer Zustand ist. Ihren Ursprung allein in den mütterlichien Organismus zu verlegen, wäre wohl verfehlt, denn das weitere Schicksal des Neugeborenen und der Mutter spricht ja dafür, daß der Säuglingsorganismus in der ersten Lebenszeit zu einem acidotischen Zustande besonders leicht zurückkehrt, während die acidotischen Zustände bei der Frau, Urämie inbegriffen, nach der Entbindung, also nach dem Zusammenleben mit dem kindlichen Organismus, nur äußerst selten vorkommen. Dieses deutet darauf hin, daß die embryonalen Lebensfunktionen am wahrscheinlichsten die hauptsächlichste Ursache der physiologischen Acidose der Gravidität sind. Und von diesem Standpunkte aus ersẹeint mir mein Befund, daß der Neugeborenenorganismus so deutliche acidotische Merkmale zeigt, nicht mehr so befremdend. Es wäre demnach hier in der acidotischen Konstitution des Neugeborenen nur eine gewisse Fortdauer der für die embryonale Zeit charakteristischen Erscheinungen zu sehen, in dem Sinne, daß die Funktionen verschiedener Organe nach der Geburt noch eine Zeitlang mangelhaft ausgebildet sind und erst allmählich aus den für die embryonale Zeit charakteristischen Abweichungen in die normale Tätigkeit übergehen. Ich habe schon in meiner Arbeit über den Icterus neonatorum ${ }^{1}$ ) zu begründen versucht, daß z. B. die Leber in.den ersten Tagen nach der Geburt vorübergehend ihre Funktionen wie die embryonale Leber ausübt. Dieselbe Rückständigkeit der Leber beim Neugeborenen könnte bei der Entstehung der acidotischen Konstitution eine große Rolle spielen. Oben habe ich gezeigt, daß das

1) Ylppö, Icterus neonatorum (inkl. Ict. n. gravis) und Gallenfarbstoffsekretion beim Foetus und Neugeborenen. Zeitschr. f. Kinderheilk. 9, 208. 1913. 
Neugeborenenblut dem Gewebe relativ weniger $\mathrm{O}_{2}$ zuführen kann als das Blut in den späteren Lebensperioden. Dadurch werden Bedingungen geschaffen, die die Entstehung saurer, abnormer, intermediärer Produkte begünstigen. Und daß im Säuglingsorganismus die Oxydationsvorgänge auch nicht in normaler Weise vor sich gehen, zeigen am schönsten die Untersuchungen von $\operatorname{Simon}^{1}$ ), der ja nachgewiesen hat, daß die $\mathrm{N}$-haltigen Körper des Urins beim Neugeborenen qualitativ ganz anders beschaffen sind als beim Erwachsenen. Die normale $\mathrm{N}$-Ausscheidung ist ganz und gar zugunsten dẹr abnormen intermediären Stoffwechselprodukte verschoben, bei denen besonders reichlich Oxyproteinsäure und Polypeptide, bis $12 \%$, in den ersten Lebenstagen ausgeschieden werden.

Dieselben acidotischen Merkmale verschwinden ja nach der Geburt verhältnismäßig rasch, nur bei einzelnen Frühgeburten existieren sie wohl längere Zeit. Diese Annahme wird besonders wahrscheinlich, nachdem, wie ich oben nachgewiesen habe, bei Frühgeburten der Urin bei Frauenmilchernährung oft monatelang eine verhältnismäßig saure Reaktion zeigte. Dies im Gegensatze zu den späteren Monaten, wo die Urinacidität in der Regel sich im weniger sauren resp. im alkalischen Gebiete bewegte. Aber der acidotische Zustand ist immer noch, wenn auch in latenter Form vorhanden und kann künstlich leicht ausgelöst werden. Das beweisen die Hungerversuche, in denen es mir gelungen ist, neben der stark sauren Reaktion des Urins in allen Fällen eine starke Verschmälerung der $\mathrm{CO}_{2}$-Regulationsbreite zu zeigen. In einem Falle sogar eine echte Acidosis mit vermehrter $\left(\mathrm{H}^{\prime}\right)$ des Blutes. Die Befunde beweisen, daß man im Säuglingsorganismus durch Hunger sehr leicht den gleichen acidotischen Zustand hervorrufen kann, den er bei der Geburt gezeigt hat. Beim Erwachsenen läßt sich etwas Ähnliches durch Hunger viel schwerer erzeugen und eine direkte echte Acidosis ist wohl noch nie bei ihm nachgewiesen worden.

Nun zur Frage der Intoxikation! In dieser haben wir ja auch. einen acidotischen Zustand kennengelernt. Wir haben regelmäßig auf dèr Höhe der toxischen Symptome eine verschmälerte $\mathrm{CO}_{2}$-Regulationsbreite, bisweilen auch eine echte Acidose mit vermehrter (H') des Blutes und immer eine herabgesetzte $\mathrm{O}_{2}$-Bindung des Blutes $\mathrm{u}$. a. typische Symptome der Acidose gefunden. Ist die Acidose hier wie beim

1) Simon, Zur Stickstoffverteilung im Urin des Neugeborenen. Zeitschr. f. Kinderheilk. 2, 1. 1911. 
Hunger nur ein Symptom oder ist sie die Ursache der Intoxikation? Diese Kardinalfrage liegt zur Beantwortung vor. Ehe ich auf sie genauer eingehe, muß ich zunächst erwähnen, was ich hier und in allem Vorangehenden unter Intoxikation verstehe. Ich bezeichne darunter lediglich den sog. toxischen Symptomen komplex, dessen einzige sichere Merkmale eine mehr oder minder akut einsetzende Bew ußtseinsstörung, die nicht auf gewöhnlichen bakteriellen Meningitiden beruht und weiterhin ein besonderer tiefer ,toxischer" A temtypus sind. Diese Intoxikation kann sowohl durch infektiöse, alimentäre

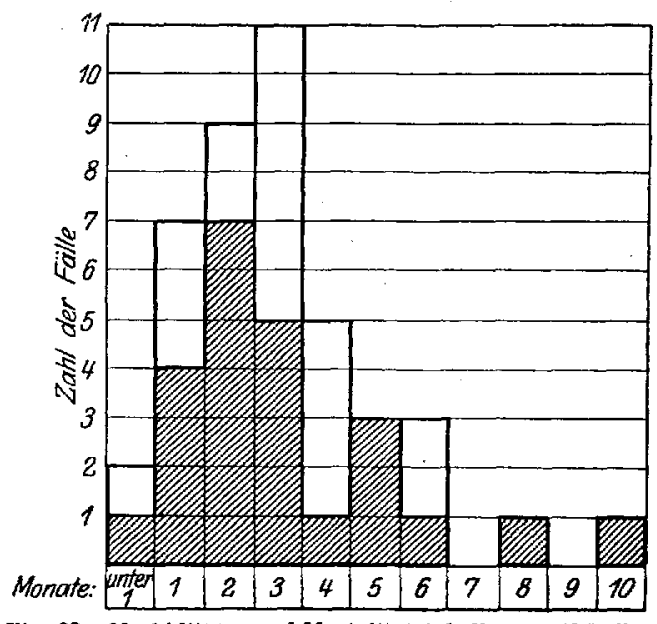

Fig. 82. Morbiditäts- und Mortalitătstabelle von 42 Făllen von Intoxikation. Schraffiert: Anzahl der Sterbefälle. wie auch thermomechanische (Hitze usw.) Noxen ausgelöst werden. Weil ich es in den einzelnen Fällen von Intoxikation für unmöglich halte, nur aus der Vorgeschichte und dem klinischen Bilde den genaueren Anteil einzelner von diesen Noxen zu bestimmen, so vermeide jich hier alle weiteren Attribute für das Wort Intoxikation. Ich kann nicht ohne weiteres Finkelstein ${ }^{\mathbf{1}}$ ) zustimmen, der die Mehrzahl der Intoxikationen für alimentär hält, auch wenn dabei Infektionen nachzuweisen sind, die er nur als ,,zufällig hineinspielend" betrachtet.

Was zunächst das Auftreten der Intoxikation betrifft, so weiß man ja, daß sie mit besonderer Vorliebe in den ersten Lebensmonaten vorkommt. Mangels besserer Statistik habe ich die wenigen Intoxikationsfälle, die ich während dieser Arbeit beobachtet habe (im ganzen 42), von diesem Standpunkte statistisch ausgerechnet.

Diese Fälle habe ich auf Fig. 32 eingetragen; es ist ersichtlich, daß die meisten Erkrankungen, d. h. ca. 70\% schon in den ersten 4 Liebensmonaten, absolut genommen, die meisten im 4. Lebensmonat auftreten. Von da aufwärts nimmt die Anzahl der Fälle rapid ab; am größten ist die Mortalität im 3. Monate, sehr groß ebenfalls im 2. und 1. Monat, wie klinisch bekannt. Genauere Prozentzahlen kann ich natürlich

1) Finkelstein, loc. cit., S. 278 dieser Arbeit. 
bei einer so kleinen Anzahl von Beobachtungen nicht angeben, dies genügt aber schon, um zu zeigen worauf es ankommt, nämlich zahlenmäßig zu beweisen, wie gehäuft die Intoxikation gerade in den ersten Monaten auftritt.

Dieses gehäufte Auftreten von Intoxikation in den ersten Lebensmonaten legt natürlich den Gedanken nahe, daß die Intoxikation in gewissem Z usammenhange mit der ,acidotischen Konstitution“" des Neugebore ne norganis mus steht. Dies wird um so wahrscheinlicher, wenn man bedenkt, daß beinahe alle Frühgeburten, die kleinsten ohne Ausnahme, vor dem Tode toxisch werden; dies einerlei, ob es sich um Ernährungsstörungen, um Darmerkrankungen oder um andere infektiöse Prozesse handelt. Zieht sich der Tod noch etwas länger hin, so verfallen sie leicht in einen tief komatösen Zustand. Diese Beobachtung habe ich in unserer Frühgeburtenabteilung besonders oft machen können. Und hierbei ist es ganz gleichgültig, ob die Frühgeburt vorher ausschließlich mit Frauenmilch oder Zwiemilch resp. Kuhmilch ernährt worden war. Diese kranken Frühgeburten zeigen, wie die obigen Blut- und Organuntersuchungen ergeben haben, die acidotischen Merkmale in höchster Potenz. Wenn wir uns noch erinnern, daß manche von ihnen in den ersten Monaten bei Frauenmilchernährung saureren Urin ausschieden als späterhin, so können wir wohl daran denken, daß der Frühgeburtenorganis mus noch monatelang acidotisch ist und daß der verschleierte Acidosezustand bei ausgebrochener Erkrankung wieder besonders aktuell wird und vor dem Tode hochgradige Intensität annimmt. Bei Frühgeburten können wir ungezwungenerweise einen gewissen kontinuierlichen $\mathrm{Zu}$ sammenhang mit der angeborenen acidotischen Konstitution des Neugeborenenorganismus und der bei der Intoxikation auftretenden Acidose konstruieren. Das auslösende Moment bei Intoxikation der Frühgeburten, besonders der mit Frauenmilch ernährten, ist wohl in der Mehrzahl der Fälle ein rein infektiöser Prozeß (Sepsis, Phlegmone usw.), der den Organismus leicht aus dem Gleichgewicht bringt, und eine von den Folgeerscheinungen ist das Aktivwerden der Acidose.

Weniger übersichtlich ist der Zusammenhang der acidotischen Konstitution des Neugeborenenorganismus mit der Intoxikation bei anderen Säuglingen. Da habe ich gewiß bei Kuhmilchernährung im allgemeinen saurere Urinwerte als bei Frauenmilchernährung gefunden. Ich erinnere an dieser Stelle an die Nahrungswechselversuche, in denen dieser Umstand besonders klar zutage trat. Weiterhin ist der folgende 
Befund vom acidotischen Standpunkte interessant, nämlich: daß bei Kuhmilchernährung der Stuhl eine alkalische Reaktion zeigte, im Gegensatz zu den Frauenmilehstühlen, bei denen ich eine saure Reaktion gefunden habe, bei gleichzeitigem umgekehrtem Verhältnis für den Urin. Es spricht dies dafür, daß die sauren Komponente des Darminhalts bei Kuhmilchnahrung verhältnismäßig mehr neutralisiert werden als bei Brustmilchnahrung, was möglicherweise auf Kosten der sonst zur Resorption stehenden Alkalimengen vor sich geht.

Wenn man weiter daran denkt, daß im Gegensatz zur Frauenmilchernährung bei Kuhmilchernährung im Urin bedeutend mehr Phosphorsäure [Moll1 $)$, zuletzt Ka miner und Ma yerhof $\left.\left.{ }^{2}{ }^{2}\right)\right]$, die ja auch ihrerseits Alkali bindet, ausgeschieden wird, so könnte man hierbei an eine gewisse chronische Alkaliarmut des Organismus bei Kuhmilchernährung glauben [Steinitz $\left.\mathrm{z}^{3}\right)$.

Im Hungerversuch zeigte sich ferner, daß das Kuhmilchkind viel größere Neigung zur Acidose hat als das Flaschenkind. Diese Befunde sind bemerkenswert, wenn man sie in Zusammenhang mit der Tatsache bringt, daß gerade die Flaschenkinder in erster Linie der Intoxikation anheimfallen. Inwieweit aber vor Entstehung der Intoxikation bei jedem Flaschenkinde sichere acidotische Merkmale im Blute vorhanden sind, steht nicht fest. Bei chronisch kranken Kindern habe ich gewiß eine deutliche Verschmälerung der $\mathrm{CO}_{2}$-Regulationsbreite, wenn auch nicht konstant, nachgewiesen. Weil ich aber kein Kind vor der Entstehung der Intoxikation daraufhin habe untersuchen können, habe ich keine strikten direkten zahlenmäßigen Beweise über diesen wichtigen Punkt an der Hand. Die klinische Erfahrung ist aber imstande, eine wertvolle Ergänzung in dieser Hinsicht zu erbringen.

Wir wissen ja, daß die Intoxikation in der Mehrzahl der Fälle mit großen Gewichtsverlusten einhergeht und außerdem, daß sie mit besonderer Vorliebe die chronischkranken ,dekomponierten“ Säuglinge trifft. Diese Gewichtsverluste sind sowohl auf verminderte Nahrungsaufnahme wie eine Einschmelzung der Körpersubstanz selbst zurückzuführen,

1) Moll, Die klinische Bedeutung der Phosphorausscheidung im Harn beim Brustkind. Jahrb. f. Kinderheilk. 69, 129, 304, 450. 1909.

2) Kaminer u. Mayerhofer, Über den klinischen Wert der Bestimmung des anorganischen Phosphors im Harn unnatürlich ernährter Säuglinge. Zeitschr. f. Kinderheilk. 8, 24. 1913.

$\left.{ }^{3}\right)$ Steinitz, loc. cit., S. 276, Anm. 3 dieser Arbeit. 
wie es L. F. Meyer ${ }^{1}$ ) und in besonders eingehender und beweisender Weise $\mathrm{Junde} \mathrm{I}^{2}$ ) nachgewiesen haben. Die Gewichtsabnahmen, die oft ganz gewaltigen Umfang erreichen, beweisen, daß der Körper schon vor dem Eintreten des toxischen symptomenkomplexes sich in einem hochgradigen, besonders akut entstandenen $\mathrm{H}$ ungerzustand befindet, der nachweislich ein acidotischer $\mathrm{Zustand}$ ist.

Man fragt sich nun, tritt der toxische Symptomenkomplex infolge der vorhandenen Acidose ein, oder wird er durch andere hypothetische Gifte eingeleitet? Durch Tierversuche ist bekannt, daß ein Symptomenkomplex wie der toxische einfach und allein durch Ubersäuerung des Blutes hervorgerufen werden kann, dessen Auftreten in hohem Maße von der Wasserstoffionenkonzentration des Blutes abhängig ist, wie dies am besten die Säurevergiftungsversuche von A. Szili ${ }^{3}$ ) beweisen.

$\mathrm{Daß}$ die obigen Verbältnisse für den Säuglingsorganismus gelten, ist natürlich klar. In den Fällen von Intoxikation, in denen ich eine echte Acidose mit vermehrter Wasserstoffionenkonzentration des Blutes gefunden habe, könnte man ja ohne weiteres daran denken, daß die direkte Säuerung des Blutes auch die Bewußtseinsstörung hervorgerufen hatte. Eine Acidose mit vermehrter Wasserstoffionenkonzentration des Blutes habe ich aber nur in den schwersten Intoxikationsfällen beobachtet und es ist sehr wahrscheinlich, daß sie erst im Verlaufe der Intoxikation entstanden ist und demnach hierbei nicht als Beweis gelten kann. In allen Hungerversuchen aber und in allen Fällen von Intoxikation habe ich dagegen eine Acidose mit verschmälerter $\mathrm{CO}_{2}$ Regulationsbreite und dementsprechend eine vergrößerte GrundWasserstoffzahl gefunden. Und was hier besonders wichtig: wo ich nur bei toxischen Kindern die WasserstoffzahI des L u mbalpunktates untersucht habe, haben sich die Werte eigentümlicherweise sehr den Werten der Grund-Wasserstoffzahl genähert. Hier war ein gewisses interessantes Abhängigkeitsverhältnis zu konstatieren, wie es aus einigen Beispielen der folgenden Tabelle klar ersichtlich ist.

Beim gesunden Kinde ist $P_{\mathbf{H}}$ des Lumbalpunktates, $c a=8,3-8,4$. und dementsprechend der Grund-Wasserstoffexponent $P_{H}=$ ca. 8,4 . Bei den toxischen Säuglingen waren diese beiden Zahlen bedeutend kleiner und näherten sich ebenfalls einander. Beide zeigten annähernd

1) L. F. Meyer, Zur Kenntnis des Stoffwechsels bei den alimentären Intoxikationen. Jahrb. f. Kinderheilk. 65, 585. 1907.

2) Jundell, loc. cit., S. 279 dieser Arbeit.

a) A. Szili, Experimentelle Untersuchungen über Säureintoxikation. Archiv f. d. ges. Physiol. 115, 82. 1906.

Zeitschrift für Kinderheilkunde. 0. XIV. 
Arvo Ylppö: Neugeborenen-, Hunger-

Die Acidität des Lumbal- bezw. Gehiln-

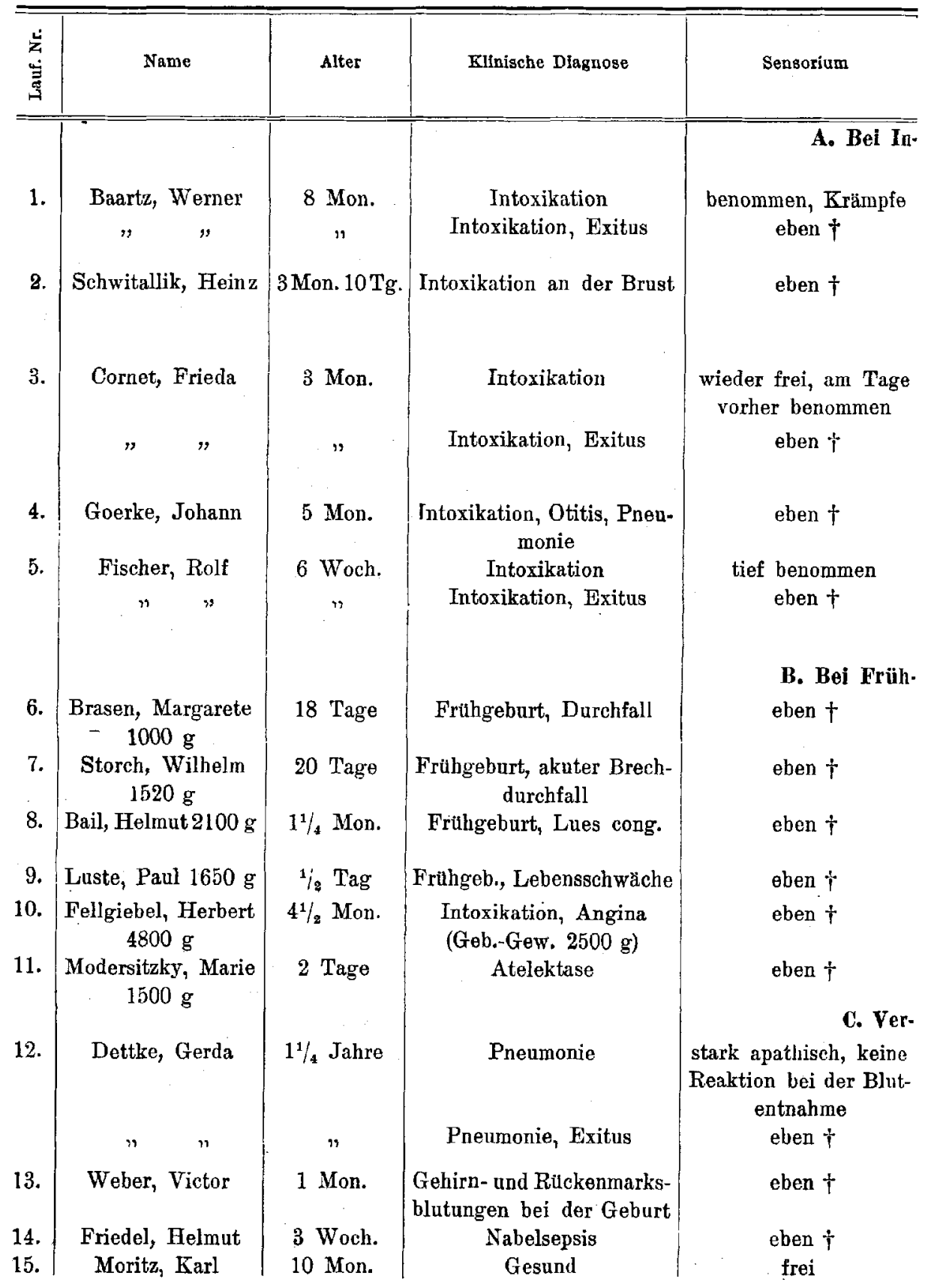


punktates und des Blutes in $\mathbf{P}_{\mathbf{H}}$.

\begin{tabular}{|c|c|c|c|c|c|}
\hline Datum & $\begin{aligned} & \mathbf{a}=\text { Lumbalpunktat } \\
& \text { oder } \\
& \mathbf{b}=\text { Gehirnpunktat } \\
& \text { Beschaffenbeit }\end{aligned}$ & $\underline{\mathbf{P}_{\mathbf{H}}}$ & \begin{tabular}{|r|}
$\mathbf{B}$ \\
Regulierter \\
Wasserstoff- \\
exponent \\
$\mathbf{P}_{\mathbf{H}}$ \\
\end{tabular} & $\begin{array}{l}\text { Grund- } \\
\text { wasserstoff- } \\
\text { exponent } \\
\mathbf{P}_{\mathbf{H}} \\
\end{array}$ & Bemerkungen. \\
\hline \multicolumn{6}{|c|}{ toxikationen. } \\
\hline 1. VII. & $a=$ klar, Eiweiß vermehrt & $7,7 \overline{\mathrm{s}}$ & 7,44 & 7,82 & \\
\hline 3. VII. & $\mathrm{b}=$ leicht opaleszierend & 6,79 & 6,61 & 7,28 & $\begin{array}{l}\text { Sektion: Enteritis hae- } \\
\text { morrh. }\end{array}$ \\
\hline 19. VII. & $\begin{array}{c}b=m . \text { Gehirnsubstanz } \\
\text { 1) ungekocht } \\
\text { 2) gekocht }\end{array}$ & $\begin{array}{l}7,09 \\
7,19\end{array}$ & 6,59 & 6,97 & $\begin{array}{l}\text { Sektion: Enteritis hae- } \\
\text { morrh., Pleuritis ad- } \\
\text { haesiva }\end{array}$ \\
\hline 8. XIr. & $\mathrm{a}=\mathrm{klar}$ & 7,98 & 7,64 & $\longrightarrow$ & Sektion: Atrophia univ., \\
\hline 14. XII. & $b=$ leicht opaleszierend & 6,85 & 6,38 & 6,95 & $\begin{array}{l}\text { Bronchitis chron., } \mathrm{Ne}- \\
\text { phritis. }\end{array}$ \\
\hline 26. VIII. & $\mathrm{b}=\mathrm{klar}$ & 7,36 & 7,10 & - & $\begin{array}{l}\text { Sektion: Bronchopneu- } \\
\text { monie, Nephritis. }\end{array}$ \\
\hline $\begin{array}{c}20 . \mathrm{V} \cdot 16 \\
n\end{array}$ & $\begin{array}{c}a=k J a r \\
b=\mathrm{m} . \text { Gehirnsubstanz }\end{array}$ & $\begin{array}{l}7,81 \\
6,80\end{array}$ & $\begin{array}{l}7,17 \\
6,80\end{array}$ & $\begin{array}{l}7,81 \\
7,22\end{array}$ & $\begin{array}{l}\text { Sektion: Enteritis, hae- } \\
\text { morrh. mit Darmge- } \\
\text { schwuren. }\end{array}$ \\
\hline \multicolumn{6}{|l|}{ geburten. } \\
\hline 22. $\mathrm{X}$. & 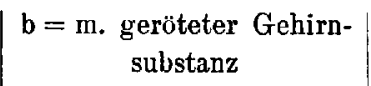 & 6,00 & 6,45 & 6,58 & $\begin{array}{l}\text { Sektion: Enteritis, Ate- } \\
\text { lektase. }\end{array}$ \\
\hline 24. X. & 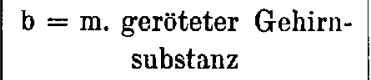 & 6,11 & 6,23 & - & - \\
\hline 30. $\mathrm{X}$. & $\mathrm{b}=$ trube Flüssigkeit & 6,31 & 6,29 & 6,60 & $\begin{array}{l}\text { Sektion: Lues cong. } \\
\text { Aplasie der Nieren. }\end{array}$ \\
\hline 30. VIII. & $\mathrm{b}=\mathrm{m}$. Gehirnsubstanz & 6,33 & 6,34 & - & - \\
\hline 29. VIII. & $\mathrm{b}=\mathrm{m}$. Gehirnsubstanz & 6,18 & 6,37 & 一 & 一 \\
\hline $\begin{array}{r}\text { 17. IX. } \\
\text { schiedenes }\end{array}$ & $\mathrm{b}=$ leicht getrübt, gelblich & 6,65 & 6,66 & - & $\begin{array}{l}\text { Sektion: Atelektase, } \\
\text { Enteritis haemorrh. }\end{array}$ \\
\hline 9. XI. & $\left\{\begin{array}{l}a=\text { leichtes Spinnwebege- } \\
\text { rilst, sonst klar } \\
\quad b=\text { leicht trube }\end{array}\right.$ & $7,0 t$ & 7,49 & 8,10 & $\begin{array}{l}\text { Sektion: Pneumonie. } \\
\text { Ödeme der Gehirn- } \\
\text { häute. }\end{array}$ \\
\hline 23. IX. & $\mathrm{b}=$ etwas getrubt & 6,74 & 6,63 & 一 & Sektion: klin. Diagnose. \\
\hline $\begin{array}{l}\text { 9. XII. } \\
\text { 3. VII. } 15\end{array}$ & $\begin{array}{c}\mathbf{b}=\text { trübe Flüssigkeit } \\
\mathbf{a}=\text { klar }\end{array}$ & $\begin{array}{l}7,79 \\
8,33\end{array}$ & $\begin{array}{l}6,71 \\
7,45\end{array}$ & $\begin{array}{l}7,97 \\
8,37\end{array}$ & $\begin{array}{l}\text { Sepsis mit Peritonitis. } \\
\text { Zwillingskind. }\end{array}$ \\
\hline
\end{tabular}


gleichgroße Verschiebungen nach der sauren Seite hin. Beim Herannahen des Todes wurde $\mathbf{P}_{\mathbf{H}}$ des Lumbalpunktates aber oft rascher sauer als $P_{\text {I }}$ für den Grundwasserstoffexponenten. Und bei Frühgeburten konnte man in der Regel Wasserstoffzahlen für das Lumbalpunktat und die Gehirnsubstanz finden, die etwa gleichgroß wie die regulierte Wasserstoffzahl waren. Oft war die Reaktion des Gehirns bei einer Frühgeburt sogar noch saurer als die des Blutes. Dies alles deutet daraufhin, da $B$ bei Frühgeburten das Zentralnervensystem dieselbe starke Neigung zur Säuerung besitzt wie das Blut und andere Organe. Dies im Gegensatz zu den älteren Säuglingen, bei denen das Zentralnervensystem eine scheinbare diesbezügliche Selbständigkeit hat, so daß die Säuerung des Blutes und der Gewebe meistens der des Lumbalpunktates bzw. der Gehirnsubstanz vorangeht. Hiernach wird es leicht verständlich, warum bei Frühgeburten so leicht der toxische Symptomenkomplex auftritt.

Es ist demnach sehr wahrscheinlich, daß bei einem Säugling ebenso, wie die Grund-Wasserstoffzahl vor dem Eintreten des toxischen Symptomenkomplexes eine Verschiebung nach der sauren Seite hin erlitten hat, auch die Wasserstoffzahl des Lumbalpunktates und wohl auch der Gehirnsubstanz selbst dieselbe Veränderung durchgemacht hat.

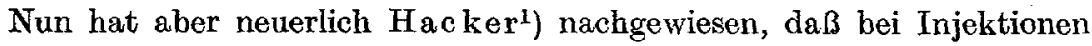
von verschiedensten Säuren die Nerven Lähmungserscheinungen zeigen, die allein nur von der Wasserstoffionenkonzentration abhängig sind. Analog verhält sich wohl auch das Zentralnervensystem des Säuglings. Es ist anzunehmen, daß auch hier bei geringgradiger Verschiebung der Reaktion nach der sauren Seite hin Lähmungserscheinungen auftreten, als deren gelindeste Form zunächst die Bewußtseinsstörung aufzufassen is $\left.{ }^{2}{ }^{2}\right) . \mathrm{Da} B$ bei Säuglingen mit akuten starken Gewichtsstürzendie Acidose neben anderen schädlichen Noxen zur Entstehung des toxischen Symptomenkomplexes in hohem Maße beitragen kann, scheint mir nach allem Vorangegangenen sehr wahrscheinlich.

Daß andererseits aber eine typische In toxikation auftreten kann,

1) Hacker, Reversible Lähmungen von Hautnerven durch Säuren und Salze. Zeitschr. f. Biol. 64 (Neue Folge 46), 224. 1914.

2) $\mathrm{Na}$ chtrag b. d. Korrektur. Inzwischen ist es mirgelungen, einen direkten Beweis hierfür zu erbringen. Nach intralumbaler Einspritzung von saurem Phosphatgemisch $\left(\mathrm{P}_{\mathrm{H}}=6,72\right)$ trat bei einem idiotischen Kinde ein typischer toxischer Zustand ein, welcher ca. 24 Stunden andauerte. Bei Kontrollversuchen mit neutralem oder leicht alkalischem $\left(\mathrm{P}_{\mathbf{H}}=7,91\right)$ Phosphatgemisch trat dagegen nur eine leichtere, bald vorübergehende Apathie ein. Die Versuche werden später veröffentlicht. 
ohne daß vorher irgendwelche acidotischen Merkmale vorhanden zu sein brauchen, ist sicher. Ich selbst verfüge über zwei solcher Fülle. In dem ersten Fall (72) handelt es sich um einen 3 Monate alten Säugling, der bisher ausschließlich Frauenmilch bekommen hatte. Das Kind erkrankte ganz plötzlich, wurde ziemlich am Anfang schon toxisch und starb unter dem Bilde der schwersten Intoxikation innerhalb 5 Stunden. Die Sektion ergab eine hämorrhagische Enteritis als Todesursache. Die Untersuchungen des Blutes und der Gewebe zeigten, daß hier beim Tode eine sichere Acidose vorlag. In dem 2. Falle handelte es sich um ein 11/4 Jahre altes, sehr kräftiges Kind (Nr. 12 der vorangehenden Tabelle), das im Verlaufe von akuter Pneumonie plötzlich toxisch wurde. Das Blut zeigte im Leben normale aktuelle Reaktion, dagegen aber eine $\mathrm{CO}_{2}$-Regulationsbreite von nur $51 \%$, und der GrundWasserstoffexponent zeigte eine deutliche Verschiebung nach der sauren Seite hin $P_{H}=8,10$. Die wahre Reaktion des klaren Liquors zeigte eine dementsprechende Vermehrung der Wasserstoffiỏnen, $P_{H}=8,09$. Hier waren vor der Entstehung des toxischenSymptomenkomplexes außer eintägigem Kranksein keine Gewichtsstürze oder andere Momente vorhanden, die die Entstehung einer Acidose hätten begünstigen können.

Wenn nun auch bei der Entstehung der Intoxikation die verschiedensten Schädigungen und Noxen je nach dem Fall die erste ursächliche Rolle spielen, so ist die große Bedeutung der Acidose im Verlaufe der Intoxikation selbst weder zu verleugnen noch zu vergessen. Dies letztere wird mehrmals getan. Deswegen will ich hier einzelne Symptome der Intoxikation in ihren Beziehungen und in ihrer Abhängigkeit mit einigen Worten berühren. Was die Bewußtseinsstörung selbst betrifft, so ist nach dem Vorangegangenen klar, daß sie so lange existiert, als eine Säuerung der Gehirnsubstanz andauert. Für die Tätigkeit des Atemzentrums, das ja die Atembewegungen und das ganze Atmen leitet, hat eine Verschiebung der Reaktion des Blutes schwere Folgen. Hasselbalch und Lundsgaard $\left.{ }^{1}\right)^{2}$ ), Douglas ${ }^{3}$ ) u. a. haben nachgewiesen, daß die Tätigkeit des Atemzentrums in sehr hohem Maße von der Wasserstoffionenkonzentration des Blutes abhängig ist. Schon ganz geringe Vergrößerung der Wasserstoffionenkonzentration

1) Hasselbalch u. Lundsgaard, Elektrometrische Reaktionsbestimmung des Blutes bei Körpertemperatur. Biochem. Zeitschr. 38, 77. 1912.

2) Hasselbalch u. Lundsgaard, Blutreaktion und Lungenventilation. Skand. Archiv f. Physiol. 27, 13. 1912.

3) Douglas, Die Regulation der Atmung beim Menschen. Ergebnisse d. Physiol. 14, 338. 1914. 
des Blutes wirkt als starker Reiz auf das Atemzentrum, das darauf mit forciertem tiefem Atmen reagiert. So könnte auch das toxische Atmen erklärt werden. Bei der Intoxikation entstehen fortwährend saure Stoffwechselprodukte, die die Reaktion des Blutes zu erhöhen versuchen. Das Atemzentrum verhindert dies eine Zeitlang dadurch, daß die Atemzüge tiefer und frequenter werden, wodurch mehr $\mathrm{CO}_{2}$ ausgeschieden und die $\mathrm{CO}_{2}$-Regulationsbreite infolgedessen verbreitert wird, wie wir es gesehen haben. Bisweilen wird sogar mehr $\mathrm{CO}_{2}$ als nötig ausgeschieden, und die aktuelle Reaktion des Blutes kann in diesen Fällen sogar alka lischer sein als in der Norm. Ähnliches haben wix auch schon oben beobachtet (siehe z. B. Fall 74 und auch einzelne Hungerversuche). Sie wurden schon als Zeichen von Uberkompensation seitens des Atemzentrums angedeutet. Vermehren sich diese sauren Stoffwechselprodukte aber fortwährend, so kann auch das forcierte Atmen für die Dauer nicht mehr die Entstehung einer echten Acidose mit Vermehrung der Wasserstoffionenkonzentration des Blutes verhindern und die Folge davon ist, daß das Atemzentrum allmählich auch gelähmt wird. Daher auch vermutlich das unregelmäßige, oft absetzende Atmen, das man in den schwersten Intoxikationen längere oder kürzere Zeit vor dem Tode oft beobachten kann. Dies mag genügen, um zu zeigen, wie enge Beziehungen und Abhängigkeitsverhältnisse zwischen de $\mathrm{m}$ A te m t y pus und den Atemstörungen einerseits und der Acidose bei der Intoxikation andererseits existieren.

An zweiter Stelle möchte ich die Zuckerausscheidung und ihr Verhältnis zu der Acidose bei Intoxikation berühren. Schon von Hof $\operatorname{ten}^{1}$ ) hatte nachgewiesen, da $B$ bei Cholera infantum Zucker im Urin auftritt. Nach ihm haben dasselbe manche andere getan, aber erst nachdem Meyer ${ }^{2}$ ) in 150 Fällen von Intoxikation ohne Ausnahme Zucker im Urin gefunden hatte, wurde diesem Befunde eine besondere Bedeutung beigelegt. Finkelstein ${ }^{3}$ ) selbst hält die Zuckerausscheidung pathognomonisch für die Intoxikation und hat sie zum Eckpfeiler seiner Lehre über alimentäre Intoxikation erhoben. Feer ${ }^{4}$ ) hat diese

1) von Hof sten, Cholera infantum pa allmänna barnhuset i. Stockholm 1887 . Ref. Centralbl. f. Kinderheilk. 1887; zit. nach Grosz, Jahrb. f. Kinderheilk. 34, 84. 1892.

2) L. F. Meyer, Zur Kenntnis des Stoffwechsels bei den alimentären Intoxikationen. Jahrb. f. Kinderheilk. 65, 585. 1907.

3) Finkelstein, Monographie loc. cit., S. 278 dieser Arbeit.

4) F'eer, Die Ernährungsstörungen im Säuglingsalter und ihre Behandlung. Beihefte zur Med. Klin. 5, 1. 1909. 
Konstanz vermißt, und ich selbst habe ebenfalls nur in ca. 65\% der Fälle Zucker im Urin nachweisen können (Nylander und Fehling I und II). Diese Prozentzahl bezieht sich sowohl auf den ersten (meistens Tee-)Tag, als auch auf die darauffolgenden Tage, wo die Nahrungsmengen je nach der Schwere der Fälle rascher oder langsamer anstiegen. Danach gehört die Zuckerausscheidung nicht unbedingt zu dem toxischen Symptomenkomplex. Die individuelle Toleranz bei Zuckerzufuhr scheint demnach auch bei Intoxikation großen Schwankungen unterworfen zu sein. Dies aber nur nebenbei. Fest steht jedenfalls die Tatsache, daß in der Intoxikation verhältnismäßig oft Zucker im Urin gefunden wird. Und unsere Frage ist nun: steht diese Zuckerausscheidung bei Intoxikation in irgendwelchem Zusammenhang mit der Acidose?

Die Intoxikation schließt in sich einen acidotischen Zustand, der sich, wie oben genauer besprochen, entweder verschleiert, nur durch die $\mathrm{CO}_{2}$-Regulationsbreite usw. feststellbar, oder offen durch Vermehrung der Wasserstoffionenkonzentrationen des Blutes zutage tritt. Andererseits wissen wir durch die neuesten Fermentuntersuchungen, besonders, durch die von Michaelis ${ }^{1}$ ) und seinen Mitarbeitern, daß alle fermentativen Prozesse ihre optimale Wirkung nur bei einer ganz bestimmten Wasserstoffionenkonzentration ausüben können. Besonders bezüglich der Glykolyse haben Rona und Wilenkoº, wie oben erwähnt, nachgewiesen, daß die Zuckerverbrennung im Organismus in besonders hohem Grade von der Wasserstoffionenkonzentration abhängig ist. Geringe Verschiebungen der Reaktion nach der sauren Richtung hin verursachen starke Herabsetzung der. Zuckerzerstörung. Dies gilt wohl auch für den'Säuglingsorganismus und demnach sind die hohen Blutzuckerwerte bei Intoxikation, die Götzky ${ }^{3}$ ) und Nie$\operatorname{mann}{ }^{4}$ ) beobachtet haben, als Zeichen von gestörter Glykolyse beim toxischen Säugling aufzufassen. Je nach der Durchlässigkeit der Nieren (für den Zucker) führt ein erhöhter Blutzuckergehalt zur Ausscheidung von, Zucker im Urin.

Daß ein unkomplizierter acidotischer Zustand allein imstande ist, eine Zuckerausscheidung im Urin hervorzurufen,

1) Michaelis, loc. cit., S. 282 dieser Arbeit.

2) Rona u. Wilenko, loc. cit., S. 340 dieser Arbeit.

$\left.{ }^{3}\right)$ Götzky, Der physiologische Blutzuckergehalt beim Kinde nach der Mikromethode von Bang. Zeitschr. f. Kinderheilk. 9, 44. 1913.

4) Niemann, Die alimentäre Glykämie des Säuglings. Jahrb. f. Kinderheilk. 83, 11. 1916 . 
zeigen schon die Versuche von Ruschhaupt'1). Er konnte bei Kaninchen nach Acetoneinatmung im Urin Zucker nachweisen und Rietschels ${ }^{2}$ ) Verdienst ist dann, die Gültigkeit dieser Versuche auch für den menschlichen Organismus bewiesen zu haben. Rietschele ${ }^{2}$ ) hat fernerhin gezeigt, daß sogar ein gesundes Brustkind bei dem ja jede chronische alimentäre Schädigung auszuschließen ist, nach 2-3 tägigem absolutem Hunger, bei nachher eingerichteten, verhältnismäßig geringen Milchmengen Zucker im Urin ausscheidet. Er führte seinen Befund auf die Sohädigungen des Darmepithels infolge von Inanition zurück. Mir scheint es aber wahrscheinlicher, daß es sich hier um eine intermediäre Störung der Zuckerverbrennung infolge von Acidose handelt. Daß beim Brustkinde während des Hungers eine Acidose (in einem Falle sogar eine „echte" Acidose mit vermehrter Wasserstoffionenkonzentration des, Blutes) entsteht, geht klar aus meinen Versuchen hervor. Daß die Herabsetzung der Zuckertoleranz und die Zuckerausscheidung während und nach dem Hunger tatsächlich a uf Utbersäuerung des Blutes und der Gewebe beruht, geht in einwandfreier Weise aus den Versuchen von Elias und Kolb $b^{3}$ ) hervor. Diese zeigen, zunächst, daß bei jungen hungernden Tieren bei Verabreichen von verhältnismäßig kleinen Glykosemengen, Hyperglykämie und Zuckerausscheidung im Urin auftritt. Und fernerhin, was uns besonders hier interessiert, daß die Hyperglykämie und Zuckerausscheidung sich durch Zufuhr von Alkali (Sodalösung) stets herabsetzen, in den meisten Fällen sogar coupieren ließ. Ich selbst habe gelegentlich meiner Phosphatversuche die Zuckerausscheidung nach Phosphatgabe bei einem toxischen Kinde (Føll 75) verschwinden sehen ${ }^{4}$ ).

1) Ruschhaupt, Über Acetonglykosurie. Archiv f. experim. Pathol. u. Pharmakol. 44, 127. 1900.

2) Rietschel, Inanition und Zuckerausscheidung im Säuglingsalter. Zeitschr. f. Kinderheilk. $\%, 282.1913$.

3) Elias u. Kolb, Über die Rolle der Säure im Kohlenhydratstoffwechsel. II. Mitt.: Über Hungerdiabetes. Biochem. Zeitschr. 52, 331. 1913.

4) Die Phosphatversuche, die, wie ich oben angeführt habe, eigentlich nicht in den Rahmen dieser Arbeit gehören, liegen vorläufig in sehr geringer Zahl vor. Ich hoffe aber, sie demnächst in Zusammenhang mit einer Arbeit über die Zuckerausscheidung im Säuglingsalter erweitern zu können, die ich aus mannigfachen Gründen einer eingehenderen Prüfung unterziehen will. Tch möchte nur voraussehicken, daB ich hier absichtlich vermieden habe, etwas über die Natur des ausgeschiedenen Zuckers zu sagen. Die allgemeine Meinung geht ja, nachdem Lang stein und Steinitz im Urin schwer magendarmkranker Säuglinge nur Lactose und Galaktose gefunden hatten (Lactase und Zuckerausscheidung bei magen- 
Es scheint mir nach alledem, daß die herabgesetzte Toleranz für Zucker und die Zuckerausscheidung in Zusammenhang mit der Acidose bei Intoxikation zu bringen sind ${ }^{1}$. Sobald wir die Zuckerausscheidung, wenn auch nur zum Teil, als Folgeerscheinung von Acidose auffassen, büßt sie ihre Bedeutung ein, als Richtschnur bei unserem ernährungstherapeutischen Vorgehen bei Intoxikationen zu dienen. Denn ernähren wir den toxischen Säugling längere Zeit sehr knapp, so befördert dieser relative Hungerzustand die Acidose, die ihrerseits die Zuckertoleranz herabsetzt und die weitere Zuckerausscheidung im Urin dadurch begünstigt. Es lockt mich in diesem Zusammenhang die Zuckerausscheidung, die ich nicht so selten bei Neugeborenen in den ersten Lebenstagen gefunden habe ${ }^{2}$ ), mit derselben bei Intoxikation in Parallele zu ziehen. Der Neugeborenenorganismus zeigt ja gerade in den ersten Tagen einen acidotischen Zustand, der nicht nur auf Hunger beruht und der auch hier in ähnlicher Weise wie die Acidose bei Intoxikation die Zuckerausscheidung begünstigt. Die Be-

darmkranken Säuglingen. Beiträge z. chem. Physiol. u. Pathol. 7, 575. 1906) dahin, daß dies die einzig ausgeschiedenen Zúckerarten sind. Mir scheint es aber sehr wahrscheinlich, daß neben Galaktose auch der andere Komponent des Milchzuckers: Glykose bei Säuglingen in den in Frage kommenden Zuständen zur Aussoheidung kommt. Auch Prof. Langstein hat sich mir gegenüber dahin geäußert, daß die Identifizierung der Zuckerarten mit Hilfe der Osazone in den kleinen zur Verfügung stehenden Mengen sehr unsicher işt. Auch die Gärungsprobe läßt uns hier manchmal im Stich.

1) Nachtrag bei d. Korrektur: Arneth berichtet im eben erschienenen Heft der Deutsch. Med. Wochenschrift (3. August 1916; S. 937), daß er bei schweren Fällen von Flecktyphus neben der großen Atmung auch eine mehrere Tage lang andauernde Zuckerausscheidung im Urin vorgefunden hat. Er ver. mutet hierbei eine gleichzeitige Gifteinwirkung, sowohl auf das Respirationswie auf das Zuckerzentrum, die ja beide benachbart im verlängerten Mark liegen. Bei Intoxikation der Säuglinge, wo wir analoge Verhältnisse vorfinden, kann man nach allem Vorangegangenen diese ,Giftwirkung“ in erster Linie in dem acidotischen Zustande des Körpers bei Intoxikation suchen.

2) Die Zuckerausscheidung im Neugeborenenurin scheint in der pädiatrischen Literatur nicht genügend bekannt zu sein. Hoeniger (Über die ephemere traumatische Glykosurie bei Neugeborenen. Deutsche med. Wochenschr. 3r, 500. 1911) hat boi 4 Kindern nach Zangengeburt Zuckerausscheidung beobachtet, bei anderen Neugeborenen aber nicht und glaubt sie auf das Trauma zurückführen zu müssen. Siehe auch v. ReuB, Die Krankheiten des Neugeborenen. Enzyklopädie der klin. Medizin. Berlin 1914 (Springer). Heller hat bei gelegentlichen Versuchen keinen Zucker im Neugeborenenurin nachweisen können (Zeitschr. f. Kinderheilkunde 13, 134. 1916). Weitere Angaben über die Zuckerausscheidung beim Neugeborenen habe ich nicht gefunden. 
kämpfung der Zuckerausscheidung muß demnach mit der Bekämpfung der Acidose zusammenfallen. In dem oben angegebenen Phosphatgemisch, das durch seine Regulationsfähigkeit imstande ist, die normale Reaktion des Organismus aufrechtzuerhalten, scheint mir ein Weg zu diesem Ziele angezeigt zu sein.

Auch die Frauenmilchtherapie kommt hierdurch in ein besonders interessantes Licht. Seit alters her hat man in der Franenmilch die letzte Rettung bei der Intoxikation gesucht. Nun habe ich ja oben gezeigt, daß bei Frauenmilchernährung der Körper bestrebt ist, die sauren Produkte der Nahrung durch den Darm auszuscheiden, was man wohl aus der konstant sehr hohen Wasserstoffionenkonzentration der Stühle bei Frauenmilchernährung schließen darf. Die alkalischen Bestandteile der Nahrung werden aber resorbiert, wie dies aus der auffallend niedrigen Wasserstoffionenkonzentration des Urins, dessen Reaktion sich meistens im alkalischen Gebiete bewegte, hervorgeht. Die Frauenmilchernährung wirkt demnach deutlich, ,antiacidotisch“. Sie schließt hiermit eine gewisse Alkalitherapie in sich, und wir können von diesem Standpunkte die guten Erfolge bei Frauenmilch - bei einer zuckerreichen Nahrung! - uns wenigstens teilweise verständlich machen. Inwieweit hier noch der Umstand eine Rolle spielt, daB die sauren Zucker-(auch Fett-)abbauprodukte, die für den Organismus als Gift wirken sollten in der Hauptsache ohne neutralisiert zu werden, schon durch den Darm ausgeschieden werden, lasse ich dahingestellt.

Zum Schluß möchte ich noch mit einigen Worten über die Rolle der Leber als mitwirkender Faktor bei der Intoxikationsacidose sprechen. Bei den Untersuchungen über die Reaktion der Gewebe fand ich ja mit einer ziemlichen Regelmäßigkeit, daß die Acidität der Leber größer als die der anderen Organe war. Weil Galle, die ja nur von der Leberzelle produziert wird, annähernd dieselbe saure Reaktion zeigte, so glaube ich mich nicht irren zu können, wenn ich diesen Befund nicht allein auf eine postmortale Säuerung, sondern auch auf vitale Verhältnisse übertrage. Bei der Intoxication würde demnach schon im Leben in der Leber eine starke Säuerung stattfinden. Der Zucker- und Glykogenabbau leiden deswegen stark, es werden reichliche Mengen von Acetonkörpern gebildet, Körper, die nach Embden und Kalberlah ${ }^{1}$ ) überhaupt nur in der Leber gebildet werden können und die in besonderer Weise zur Entstehung der Acidose beitragen.

1) Embden v. Kalberlah, loc. cit., S. 337 dieser Arbeit. 
Ob bei dieser Begünstigung der Acidose direkte Zerstörungen oder degenerative Schädigungen des Leberparenchyms selbst, die ja bei magendarmkranken Kindern nach Thiemich) besonders häufig und speziell bei Intoxikation wohl immer vorhanden sind, ausschlaggebend sind, oder ob dabei die verminderte $\mathrm{O}_{2}$-Zufuhr, die ich durch die Untersuchungen der $\mathrm{O}_{2}$-Dissoziationskurve des Blutes habe nachweisen können, in Frage kommen, kann ich nicht entscheiden. Aber das ist auch nebensächlich. Die Hauptsache ist, daß hiermit die Leber als der wichtigste Ort für die Produktion der sauren Stoffwechselprodukte bei der Intoxikation anzusehen ist ${ }^{2}$ ). Hierin wird nichts dadurch geändert, dass bei Kuhmilchernährung ausserdem noch vermehrte Mengen von sauren Stoffwechselprodukten, besonders der Phosphorsäure, ausgeschieden werden.

Interessant ist in diesem Zusammenhange, daß Fischler ${ }^{3}$ ), ein sehr verdienter Forscher auf dem Gebiete der Leberpathologie, nunmehr sich gezwungen sieht, die Leber für die Fleischintoxikation bei den $\mathrm{Hunden}$ mit Eckscher Fistel verantwortlich zu machen. $\mathrm{Zu}$ dieser Meinung $\mathrm{kam}$ er, nachdem es ihm gelang, die Fleischintoxikation nur dann regelmäBig hervorzurufen, wenn er die Hunde vorher einige Tage hungern ließ und gleichzeitig Phlorrhizin einreichte. Dieser Hinweis über die jetzige Auffassung von der Fleischintoxikation scheint mir hier erwähnenswert in Anbetracht der Analogie des toxischen Symptomenkomplexes bei Fleischintoxikation und Säuglingsintoxikation. Wenn ich auch nicht so weit gehe, daß ich bei Säuglingen die Leber allein für die Entstehung des toxischen Symptomenkomplexes verantwortlich machen will, so kann ich wohl doch nach allem Obigen der Leber als dem größten parenchymatösen Organ eine außerordentlich wichtige Rolle bei der Entstehung der Intoxikationsacidose zuschreiben.

1) Thiemich, loc. cit., S. 276 dieser Arbeit.

2) Es mag an dieser Stelle erwähnt werden, daß Tugendreich (Histologischer Nachweis der Acidose des Säuglings. Berl. klin. Wochenschr. 1908, S. 886) und Rott (Die Farbreaktion des Gewebes bei der Säuglingsacidose. Monatsschr f. Kinderheilk. 7, 73. 1908/09), mittels einer Fürbemethode in Leberschnitten bei Intoxikation die Acidose nachweisen wollten. Koch (Utber den mikrochemischen Nachweis der Acidose bei Ernährungsstörungen. Monatsschr. f. Kinderheilk. 8, 465. 1909/10) konnte aber ihre Angaben nicht bestätigen und betont, da $\beta$ diese Farbreaktion der Leber für irgendwelche diagnostische Zwecke nicht zu verwerten ist. Die Farbenunterschiede sind von allzu vielen technischen und anderen Zufälligkeiten abhängig.

3) Fischler, Die Hervorbringung der Fleischintoxikation beim Eckschen Fistelhunde. Deutsches Archiv f. klin. Med. 113, 530. 1914. 


\section{Zusammenfassung.}

Beim Säugling wurde ein ausgesprochen acidotischer Zustand gefunden:

l. Im Neugeborenen - Alter, d. h. bei der Geburt und in den ersten darauf folgenden Tagen; dies bei Kindern, einerlei ob frühgeboren oder ausgetragen. Aus diesem Grunde wird von einer acidotischen Konstitution des Neugeborenen gesprochen.

2. Während des Hungers.

3. Bei der Intoxikation, womit hier die Gesamtheit der Erkrankungen bezeichnet werden, die den sog. toxischen Symptomenkomplex zeigen.

Mit der Acidose wird ein Zustand bezeichnet, in welchem organische unverbrennende Säuren und deren Salze, möglicherweise auch anorganische, in vermehrten Mengen im Körper kreisen. Die Wasserstoffionenkonzentration, als Ausdruck der wahren Reaktion des Blutes und der Körpersäfte, kann bei einer Acidose, die so aufgefaßt wird, dank verschiedener Regulationsvorgänge im Körper, normal, oft aber a uch größer und in vereinzelten Fällen, vermutlich infolge von Utberkompensation durch das übermäßig gereizte Atemzentrum, kleiner als in der Norm sein. Die Bestimmung der Wasserstoffionenkonzentration, die in dieser Arbeit durch Gaskettenmethode ausgeführt wurde, allein genügt demnach nur, diejenigen acidotischen Zustände als solche zu erkennen, in denen eine tatsächlich erhöhte Wasserstoffionenkonzentration vorhanden ist. Zur Feststellung anderer acidotischer Zustände müssen weitere Wege eingeschlagen werden.

In der vorliegenden Arbeit wurden deshalb die acidotischen $\mathrm{Zu}$ stände daneben durch folgende teils neue Methoden nachgewiesen:

A. Mittels eines neuen Indicators für Acidose, der $\mathbf{C O}_{2^{-}}$ Regulationsbreite genannt wird, die in Prozenten ausgedrückt, einen Einblick über den Grad der Acidose gestattet. Zur Ermittelung dieser ist eine vorherige Bestimmung der aktuellen Reaktion und der „Grundreaktion“ des Blutes notwendig. Mit "Grundreaktion" des Blutes wird die Reaktion des $\mathrm{CO}_{2}$-freien Blutes bezejchnet.

B. Durch Bestimmung der $\mathrm{CO}_{2}$-Dissoziationskurve des Blutes, die gleichzeitig als sie als Indicator für die Acidose gilt, auch Aufschluß über die $\mathrm{O}_{2}$-Zufuhr ins Gewebe gibt. 
und Intoxikationsacidosis in ihren Beziehungen zueinander.

C. Durch Bestimmung des Wasserstoffionen-, Stoff wechsels", d. h. durch die Bestimmung der wahren Reaktion des Stuhls und des Urins neben der des BIutes unter Berücksichtigung der jeweiligen Nahrung und anderer Umstände.

D. Durch Bestimmung der wahren Reaktion verschiedenster Organe gleich nach dem Tode.

Auf diesem Wege wurde zur Begründung der drei ersten Behauptungen folgendes festgestellt:

ad 1. Sowohl beim frühgeborenen als a usgetragenen Neugeborenen ist die $\mathrm{CO}_{2}$-Regulationsbreite schon bei der Geburt merkbar und in den ersten darauf folgenden Tagen bedeutend kleiner als später. Der Urin ist hier (bei genügender Frauenmilchernährung) meistens saurer als später, besonders bei ganz kleinen Frühgeborenen. Bei diesen bleibt der Urin oft 2-5 Monate lang auffallend saver, um erst dann allmählich bei gleichbleibender Frauenmilchernährung zu den normalen Werten aufzusteigen.

Die $\mathrm{O}_{2}$-Dissoziationskurvezeigt bei Neugeborenen und Frühgeburten einen auffallend niedrigen Verla uf. Die gleiche Menge von Hämoglobin kann demnach hier dem Gewebe weniger $\mathrm{O}_{2}$ zuführen als normalerweise.

Die Organe der an verschiedenen Krankheiten verstorbenen Frühgeburten zeigen im allgemeinen saurere Werte als die der anderen Säuglinge, mit Ausnahme der an schwerer Intoxikation zugrunde gegangenen.

ad 2. Beim Hunger wurde eine starke Verschmälerung der $\mathrm{CO}_{2}$-Regulationsbreite festgestellt; bei Kuhmilchkindern eine noch intensivere als bei Brustmilchkindern. Bei einer kleinen Frühgeburt wurde hierbei sogar eine deutliche Vermehrung der Wasserstoffionenkonzentration im Blute nachgewiesen. Der Urin wird beim Hunger stark sauer.

ad 3. In den schwersten Fällen von Intoxikation ist die Wasserstoffionenkonzentration meistens schon im Leben, ständig kurzvordem Todedeutlich vermehrt. Bei den in Heilung übergegangenen Fällen, mit Ausnahme von einem, ist sie normal, bisweilen sogar kleiner (Blut alkalischer!) als in der Norm. Dagegen ist die $\mathrm{CO}_{2}$ Regulationsbreite bei allen Intoxikationen stark 
verschmälert und wird beim Herannahen des Todes besonders schmal; gleichzeitig beginnt die Wasserstoffionenkonzentration sich besonders rapid zu vermehren (siehe genauer Fig. 33).

Die $\mathrm{O}_{2}$-Dissoziationskurve des Blutes hat bei Intoxikation immer einen niedrigeren Verlauf als in der Norm. Bei leichteren Fällen ist die Abweichung geringer, bei schweren oft sehr bedeutend, kurz vor dem Tode aber erst besonders hochgradig, so daß das Blut unter bestimmten $\mathrm{O}_{2}$-Partialdrucken kaum $1 / 3$ yon der normalen $\mathrm{O}_{2}$ Menge aufnehmen kann. Bei Intoxikation ist die $\mathrm{O}_{2}$ Zufuhr, wie das Gewebe, demnach herabgesetzt, und hierdurch sind die Bedingungen zu verschiedensten $0 x y-$ dationsstörungen gegeben.

Im Herzblut, das auch die Verhältnisse unmittelbar vor dem Tode charakterisiert, waren diese Veränderu ngen des Blutes bei Intoxikation besonders hochgradig, merkbar größer als bei den an anderen Krankheiten verstorbenen Säuglingen, nur die Frühgeburten ausgenommen.

Die wahre Reaktion verschiedener Gewebe ist bei Intoxikation in der Regel mehr sauer als bei anderen Erkrankungen; die Reaktionswerte gleichen denen bei Frühgeburten.

Die Intoxikationsacidose kann a us der Neugeborenenund Hungeracidose wenigstens teilweise abgeleitet werden. Die Neugeborenenacidose kann hierbei als eine noch vorhandene latente acidotische Disposition wirken. Treten doch die meisten Intoxikationen in den ersten Lebensmonaten auf. Von den bei dieser Arbeit beobachteten bis ca. $70 \%$ der Fälle in den ersten 4 Monaten. Die Hungeracidose wirkt als tatsächlicher beitragender Faktor bei der Entstehung der Intoxikationsacidose in der großen Gruppe der Intoxikationen, die mit beträchtlichen Gewichtsstürzen einhergehen.

In dieser Gruppe, die die Mehrzahl der Intoxikationen u mfabt, spielt die Acidose eine gewisse ursächliche Rolle bei der Entstehung des toxischen Symptomenkomplexes.

Es kommen aber, wenn auch seltener, ganz plötzlich mitten in voller Gesundheit einsetzende Intoxikationen ovor, wo die Acidose 


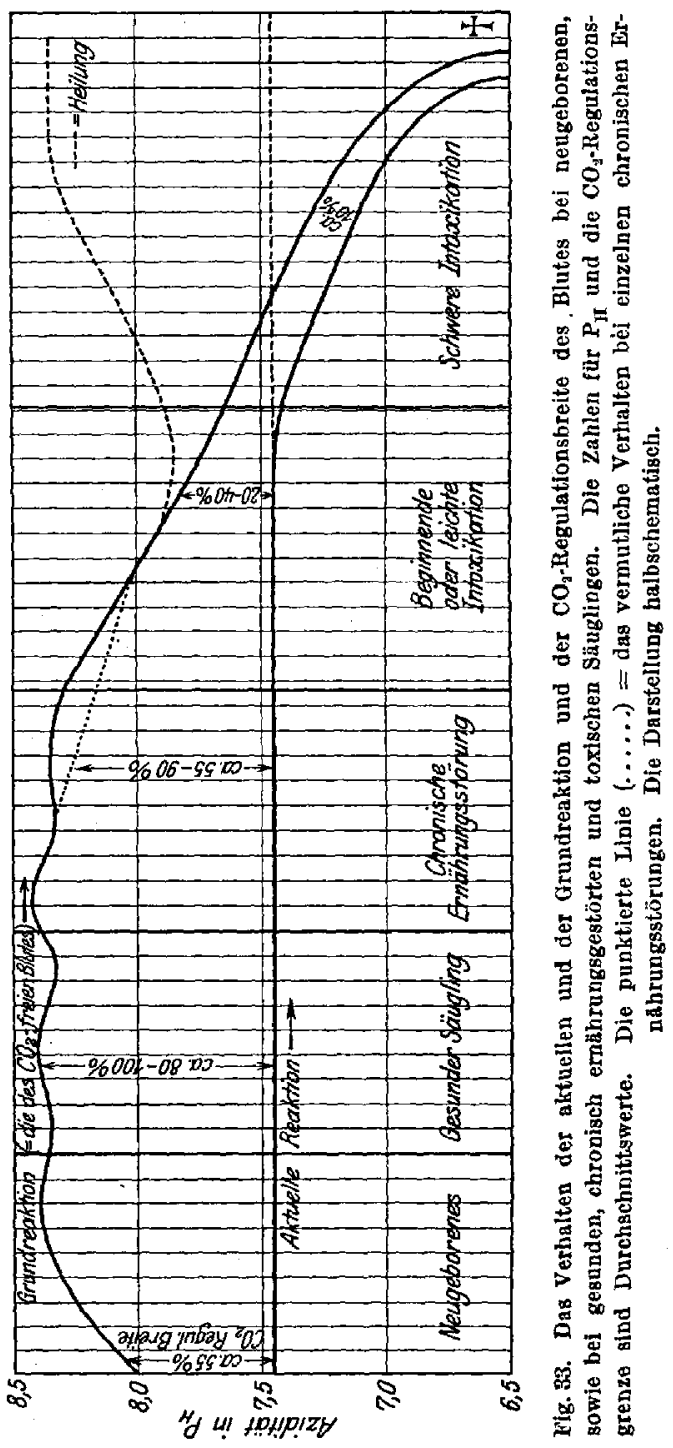


erst nach dem Auftreten des toxischen Symptomenkomplexes als eine Folgeerscheinung einsetzt.

Die wahre Reaktion der Leber wurde bei Intoxikationen in der Regel relativ saurer als die der anderen Organe gefunden. Weil die Galle, ein Produkt der lebenden Leberzelle, meistens annähernd dieselbe Wasserstoffionenkonzentration hatte wio die Leber kurz nach dem Tode, so wird es für sehr wahrscheinlich gehalten, daß diese relativ saure Reaktion des Lebergewebes schon im Leben existiert. Aus diesen und anderen Gründen wird die Leber als wichtigster Entstehungsort der sauren Stoffwechselprodukte bei Intoxikation angesehen.

Es werden ferner die Beziehungen der Acidose selbst mit einigen Einzelsymptomen der Intoxikation besprochen. Hierbei wird besonders betont, daß die Zuckerausscheidung im Urin in erster Linie von dem acidotischen Zustande des Körpers bei der Intoxikation abhängig ist.

Zur Bekämpfung der Acidose bei Intoxikation wird ein besonderes Phosphatgemisch vorgeschlagen, das bestrebt ist, eine normale Körperreaktion gegenüber der. Säuerung aufrechtzuerhalten.

Weiter wird darauf hingewiesen, daß die Frauen milchernährung in gewissem Sinne auch eine, ,antiacidotisehe" Therapie in sich schließt.

Bei Frauenmilchernährung werden nämlich viel saure Produkte durch den Darm als nicht neutralisiert ausgeschieden, worauf eine konstant vorkommende saure Reaktion der Stühle (hohe Wasserstoffionenkonzentration) deutet. In den Kreislauf aber werden bei Frauenmilchernährung hauptsächlich nur basische Bestandteile aufgenommen; denn der Urin zeigt hier meistens eine Reaktion, die sich im alkalischen Gebiete bewegte. Dagegen ist das Verhältnis bei den gewöhnlichen Kuhmilchmischungen in der Regel umgekehrt, wie es am deutlichsten beim Nahrungswechsel beobachtet werden konnte. Bezüglich der Ausscheidung der freien Wasserstoffionen des „Wasserstoffionenstoffwechsels" bestehen demnach zwischen Darm und Niere vikarierende Wechselbeziehnngen.

Welcher von diesen Ausscheidungswegen in erster Linie in Anspruch genommen wird, hängt bei Nahrungszufuhr von der Art der Nahrung ab. In Hungerzuständen fällt die Ausscheidung der freien Wasserstoffionen ziemlich ausschließlich der Niere zu. 
Herrn Professor Langstein will ich wieder an dieser. Stelle für seine liebenswürdige Unterstützung und sein reges Interesse an dieser Arbeit meinen besten Dank aussprechen.

\section{Literatur-Verzeichnis.}

(Bezieht sich nur auf Arbeiten, die in direktem Zusammenhang mit der Acidose. frage stehen. Andere im Text erwähnte Arbeiten siehe nur Fußnote.)

Araki, Über die Bildung von Milchsäure und Glykose im Organismus bei Sauerstoffmangel. III. Mitt. Zeitschr. f. physiol. Chemie 16, 45’. 1892.

Aron Hans u. Marianne Franz, Organische Säuren im Säuglingsharn. Monatsschr. f. Kinderheilk. 12, 645. 1914.

Aschenheim, Ưber Zuckerausscheidung im Kindesalter. Verhandl. d. Gesellsch. f. Kinderheilk., Salzburg 1909, S. 178.

Baginsky, Über Acetonurie bei Kindern. Archiv f. Kinderheilk. 9, 1. 1888.

Bahrdt, Zur Pathogenese der akuten Verdauungsstörung im Säuglingsalter. Verhandl. d. Gesellsch. f. Kinderheilk, Karlsruhe 1911, S. 62.

Barthez u. Rilliet, Handbuch der Kinderkrankheiten. Deutsche Ausgabe von Dr. Hagen. Leipzig 1855, I. Teil, S. 701.

Bauer, Über Lungenblähung bei alimentärer Intoxikation. Verhandl. d. Gesellsch. f. Kinderheilk., Wien 1913, S. 25.

Begu n, Herrmann u. Münzer, Über Acidosis und deren Regulation im menschlichen Körper. Biochem. Zeitschr. \%1, 255. 1915.

Bendix, Zur Frage der Ammoniakausscheidung durch den Harn bei magendarmkranken Säuglingen. Jahrb. f. Kinderheilk. 48, 165. 1898.

Benjamin, Das Blut bei den Ernährungsstörungen des Säuglings. Verhandl. d. Gesellsch. f. Kinderheilk., Cöln 1908, S. 286.

van den Bergh, Zur KenntniB der Gastroenteritis im Säuglingsalter. III. Mitt.: Einfluß von Alkalizufuhr auf die Ammoniakausscheidung. Jahrb. f. Kinderheilk. 45, 265. 1897.

Bernhard, Osmotischer Druck und Eiweißgehalt des Blutes alimentär intoxizierter Säuglinge. Dissert. Leipzig 1913.

Bernheim - Karrer, Utber den Einfluß der Milchzersetzung auf die Entstehung akuter Verdauungsstörungen der Säuglinge. Zeitsohr. f. Kinderheilk. 13, 435. 1916.

Bostock, Gertrude, Zur Kenntnis der Säureintoxikation. Zeitschr. f. phys. Chemie 84, 468. 1913.

Brüning, Über die Beziehungen zwischen Lebererkrankungen und postmortaler Oxydationskraft des Lebergewebes. Monatsschr. f. Kinderheilk. 2, 129. 1903.

Camerer, Die stickstoffhaltigen Bestandteile im menschlichen Urin und die sogenannte Acidose. Monatsschr. f. Kinderheilk. 2, 1. 1903.

Mo Cleave, Th. C., Acute acid intoxication in children. Journ. of the Amer. med. Assoc. 61, 1764. 1913. Ref.: Zentralbl. f. d. ges. inn. Med. 9, 4. 1914.

Czerny, Zur Kenntnis der Gastroenteritis im Säuglingsalter. IV. Mitt.: Respirationsstörungen. Jahrb. f. Kinderheilk. 45, 271. 1897. 
Czerny u. Keller, Zur Kenntnis der Gastroenteritis im Säuglingsalter. V. Mitt.: Säurebildung. Jahrb. f. Kinderheilk. 45, 274. 1897.

- - Des Kindes Ernährung usw. II. Teil, 9. Kapitel, S. 135ff. Alimentäre Toxikosen der Kinder im ersten Lebensjahre. Leipzig und Wien 1906/09 (F. Deuticke).

Douglas, C. Gordon, Die Regulation der Atmung beim Menschen. Ergebnisse d. Physiol. 14, 338. 1914.

Elias, Uther die Rolle der Säure im Kohlenhydratstoffwechsel. Über Säurediabetes. Biochem. Zeitschr. 48, 120. 1913.

- u. Kolb, Über die Rolle der Säure im Kohlenhydratstoffwechsel. II. Mitt.: Über Hungerdiabetes. Biochem. Zeitschr. 52, 331, 1913.

Embden u. Kalberlah, Über Acetonbildung in der Leber. Beiträge z. chem. Physiol. u. Pathol. 8, 121. 1906.

Feer, Die Ernährungsstörungen im Säuglingsalter und ihre Behandlung. Beibefte zur Med. Klin. 5, 1. 1909.

Feldman u. Hill, Journ. of Physiol. 42, 439. 1911. Zit. bei Douglas, Ergebnisse d. Physiol. 14, 338. 1914.

Finkelstein, Zur Ätiologie der Ernährungsstörungen der Säuglinge. Verhandl. d. Gesellsch. f. Kinderheilk, Stuttgart 1906, S. 117.

- Über alimentäre Intoxikation im Säuglingsalter. (Monographie.) Berlin 1910 (Karger). Sonderabdruck aus dem Jahrb. f. Kinderheilk 65/68.

Fischler, Die Hervorbringung der Fleischintoxikation beim Eckschen Fistelhunde. Deutsches Arohiv f. klin. Med. 113, 530. 1914.

Fleisch, Handbuch über die Krankheiten der Kinder. 4 Bde. Leipzig 1803 (Jacobäer). Daselbst 1. Bd., S. 510ff.

Forschbach, Zur Frage der Muskelmilchsäure beim Diabetes mellitus und der glykolytischen Kraft des Muskels. Biochem. Zeitschr. 58, 339. 1913/14.

Freund, Zur Kenntnis der Oxydationsvorgänge bei gesunden und kranken Säuglingen. Verhandl. d. Gesellsch. f. Kinderheilk., Hamburg 1901, S. 187.

- Zur Kenntnis der chronischen Ernährungsstörungen der Säuglinge. II. Mitt.: Säuren und Basen im Urin kranker Säuglinge. Monatsschr. f. Kinderheilk. 1, 230. 1902.

Friedericia, Über die Bestimmung der diabetischen Acidosis durch Untersuchung der Kohlensäurespannung in der Lungenluft. Zeitschr. f. klin. Med. 80, 1.1914.

Gaethgens, Zur Frage der Ausscheidung freier Säuren durch den Harn. Vorläufige Mitteilung. Centralbl. f. d. med. Wissensch, 10, 833. 1872.

Götzk y, Der physiologische Blutzuckergehalt beim Kinde nach der Mikromethode von Bang. Zeitschr. f. Kinderheilk. 9, 44. 1913.

Griesbaoh u. Oppenheimer, Über Milchsäurebildung im Blute. Biochem. Zeitschr. 55, 323. 1913.

Hacker, Reversible Lähmungen von Hautnerven durch Säuren und Salze. Zeitsohr. f. Biol. 64, 224. 1914.

Hagner, Schwankungen im Eiweißgehalt und in der. Leitfähigkeit beim Säuglingsblute. Zeitschr. f. Kinderheilk. 8, 50. 1913.

Hallervorden, Über Ausscheidung von Ammoniak im Urin bei pathologischen Zuständen. Archiv f. experim. Pathol. u. Pharmakol. 12, 237. 1880. 
Hasselbalch, Neutralitätsregulation und Reizbarkeit des Atemzentrums in ihren Wirkungen auf die Kohlensäurespannung des Blutes. Biochem. Zeitsohr. 46, 403. 1912.

- Ammoniak als physiologischer Neutralitätsregulator. Biochem. Zeitschr. 74 , 18. 1916.

_- Die „reduzierte“" und die „regulierte" Wasserstoffzahl des Blutes. Biochem. Zeitschr. 74, 56. 1916.

- u. Lundsgaard, Blutreaktion und Lungenventilation. Skand. Archiv f. Physiol. 2r, 13. 1912.

- u. Gammeltoft, Die Neutralitätsregulation des graviden Organismus. Biochem. Zeitschr. 68, 206. 1915.

- u. Lindhard, Zur experimentellen Physiologie des Höhenklimas. IV. Biochem. Zeitschr. 74, 1. 1916.

Hecht, Das Verhalten der Fettsäurebildung im Darminhalt des Säuglings. Münch. med. Wochenschr. 57, 63. 1910.

Heoker, Periodisches Erbrechen mit Acetonämie. Periodische Acetonämie. Ergebnisse d. inn. Med. u. Kinderheilk. 7, 242. 1911.

Heim, Die Rolle der Wärmestauung und Exsiccation bei der Intoxikation der Säuglinge, Archiv f. Kinderheilk. 59, 91. 1913.

- u. John, Ưber die interne Anwendung von Salzlösungen bei Behandlung der akuten Ernährungsstörungen im Säuglingsalter. Monatsschr. f. Kinderheilk. 6, 561. 1907.

- - Die Behandlung der Exsiccation mit Salzlösungen. Jahrb. f. Kinderheilk. 70, 96. 1909.

Henderson u. Spiro, Zur Kenntnis des Ionengleichgewichts im Organismus. I. Teil: Über Basen- und Säuregleichgewicht im Harn. Biochem. Zeitschr. 15, 105. 1909.

- u. Palmer, On the several factors of acid excretion. Journ. of biol. Chem. 18, 305. 1914.

Hildebrandt, Ưber eine experimentelle Stoffwechselabnormität. Zeitschr. f. physiol. Chemie 35, 141. 1902.

Hirso hfeld, Untersuchungen über alimentäre Intoxikation in ihren Beziehungen zum sympathischen Nervensystem. Jahrb. f. Kinderheilk. r8, 197. 1913. Erg.-Heft.

Hof meister, Über Resorption und Assimilation der Nährstoffe. VI. Mitt.: Über den Hungerdiabetes. Arohiv f. experim. Pathol. u. Pharmakol. 26, 355. 1890.

Hofsten, von, Cholera infantum på allmänna barnhuset i Stockholm 1887. zit. nach Grosz; Jahrbuch f. Kinderh. 34, 84. 1892.

Howland and Me K. Marriott, Observations upon the so-called food intoxication of infants with especial reference to the alveolar air. Proc. of the Soc. for experim. Biol. and Med. 12, 51. 1914.

- - als Referenten in: A discussion of acidosis with special reference to that occuring in the diseases of childhood in the New York Academy of Medicine, Section on Pediatrics, Dec. $2^{\text {nd }}$ 1915. Ref. : Arch. of Ped. 33, 124. Febr. 1916. Hüssy, Weitere Beitrüge zur Kenntnis der Acidosis im Kindesalter. Zentralbl. f. Physiol. u. Pathol. d. Stoffwechsels, N. F. 1, 36. 1906. 
Hutinel, Les rêactions méningées dans l'azotémie chez les nourrissons. Paris méd. 4, 41. 1913.

Jaeger, Über akute Magenerweichung durch Vermehrung der Essigsäure usw. Zit. nach Barthez u. Rilliet, Handb. d. Kinderkrankheiten. Deutsche Ausgabe von Dr. Hage n. Leipzig 1855, I. Teil, S. 701.

Irisawa, Ủber die Milchsäure im Blute und im Harne. Zit. nach O. Porges, Wiener klin. Wochenschr. 24, 1147. 1911.

Jundell, Untersuchungen über den Stoffwechsel bei der Dyspepsie und der alimentären Intoxikation. Zeitschr. f. Kinderheilk. 8, 235.1913.

Kaminer u. Mayerhofer, Über den klinischen Wert der Bestimmung des anorganischen Phosphors im Harne unnatürlich ernährter Säuglinge. Zeitschr. f. Kinderheilk. 8, 24. 1913.

Keller, Zur Kenntniss der Gastroenteritis im Säuglingsalter. II. Mitt.: Ammoniakausscheidung. Jahrb. f. Kinderheilk. 44, 25. 1897.

- Utber die Bedeutung der Acidität des Harns beim magendarmkranken Säugling. Jahrb. f. Kinderheilk. 4\%, 176. 1898.

- Zur Kenntnis der Gastroenteritis im Säuglingsalter. VI. Mitt.; Einfluß der Zufuhr von Ammoniaksalzen auf die Harnstoffausscheidung. Jahrb. f. Kinderheilk. 4\%, 187. 1898.

- Das Schicksal der Amidosäuren im Organismus des magendarmkranken Säuglings. Centralbl. f. allg. Pathol. u. pathol. Anat. 9, 739. 1898.

- Malzsuppe, eine.Nahrung für magendarmkranke Säuglinge. Jena 1898 (Fischer).

- Zur Kenntnis der chronischen Ernährungsstörungen der Säuglinge. IIL. Mitt.: Fettumsatz und Acidose. Monatsschr. f. Kinderheilk. 1, 234. 1902.

Kenneway, Pembrey and Poulton, Observations on acidosis. Journ of Physiol. 4r. 1913/14. (Proc. of the Physiol. Soc., Oct. 18, 1913, p. X/XI.)

Klose, Zur Kenntnis der Körperzusammensetzung bei Ernährungsstörungen. Verhandl. d. Gesellsch. f. Kinderheilk., Wien 1913, S. 268 und Jahrb. f. Kinderheilk. 80, 154. 1914.

Kooh, Über den mikrochemischen Nachweis der Acidose bei Ernährungsstörungen. Monatsschr. f. Kinderheilk. 8, 465. 1909/10.

Koppel u. Spiro, Über die Wirkung von Moderatoren (Puffern) bei der Verschiebung des Säure-Basengleichgewichtes in biologischen Flüssigkeiten. Biochem. Zeitschr. 65, 409. 1914.

Külz, Über eine neue linksdrehende Säure (Pseudooxybuttersäure). Ein Beitrag zur Kenntnis der Zuckerruhr. Zeitschr. f. Biol. 20, 165. 1884.

Kussma u\}, Zur Lehre vom Diabetes mellitus. Deutsches Archiv f. klin. Med. 14, 1.1874.

Langstein u. Meyer, Die Acidose im Kindesalter. I. Mitt.: Die Acidose des älteren Kindes. Jahrb. f. Kinderheilk. 61, 454. 1905.

- u. Steinitz, Lactase und Zuckerausscheidung bei magendarmkranken Säuglingen. Beiträge z. chem. Physiol u. Pathol. 7, 575. 1906.

Lauritzen, Utber Acidosebestimmungen und ihre klinische Anwendbarkeit bei Diabetes mellitus. Zeitschr. f. klin. Med. 80, 13.1914.

Luzzatto, Über die Natur und Ursachen der Morphinglykosurie. Archiv f. experim. Pathol. u. Pharmakol. 52, 95. 1905. 
Magnus-Levy, Die Acetonkörper. Ergebnisse d. inn. Med. u. Kinderheilk. 1, 352. 1908. (Gute Literaturübersicht über Acetonkörper.)

Marshall, The theories of acidosis and acidosis in children. Arch. of Pediatr. 31, 121. 1914.

Martius, Konstitution und Vererbung in ihren Beziehungen zur Pathologie. Enzyklopädie der klin. Med. Berlin 1914 (Springer).

Masel, Zur Frage der Säurevergiftung beim Coma diabeticum. Zeitschr. f. klin. Med. 79, Heft 1/2. Ref. im Zentralbl. f. Physiol. 28, 181. 1914.

Meyer, Zur Kenntnis des Stoffwechsels bei den alimentären Intoxikationen. Jahrb. f. Kinderheilk. 65, 585. 1907.

- Experimentelle Untersuchungen zum alimentären Fieber. Deutsche med. Wochenschr. 35, 194. 1909.

- Die Bedeutung der Mineralsalze bei den Ernährungsstörungen des Säuglings. Verharidl. d. Gesellsch. f. Kinderheilk., Salzburg 1909, S. 1.

- u. Langstei n, Die Acidose im Kindesalter. II. Mitt.: Die Acidose des Säuglings. Verhandl. d. Gesellsch. f. Kinderheilk., Meran 1905, S. 182 und Jahrb. f. Kinderheilk. 63, 30. 1906.

- u. Rietschel, Über den Eiweißstoffwechsel bei schweren Ernährungsstörungen der Säuglinge. Verhandl. d. Gesellsch. f. Kinderheilk., Stuttgart 1906, S. 241.

Michaelis, Die Wasserstoffionenkonzentration. Ihre Bedeutung für die Bio. logie und die Methoden ihrer Messung. Berlin 1914 (Springer).

- Die Bedeutung der Wasserstoffionenkonzentration des Blutes und der Gewebe. Deutsche med. Wochenschr. 40, 1170. 1914.

- u. Kramsztyk, Die Wasserstoffionenkonzentration der Gewebssäfte. Biochem. Zeitsohr. 62, 180: 1914.

Minkowski, Über das Vorkommen von Oxybuttersäure im Harn bei Diabetes mellitus. Ein Beitrag zur Lehre vom Coma diabeticum. Archiv f. experim. Pathol. u. Pharmakol. 18, 35. 1884.

Mohr u. Staehelin, Handbuch der inneren Medizin. Bd. IV., S. 589, Kapitel: Diabetes mellitus von J. Baer. Berlin 1912 (Springer).

Morse, Acid intoxication in infance and childhood. Arch. of Pediatr., Aug. 1905; zit. bei Hecker, Ergebnisse d. inn. Med. u. Kinderheilk. 7, 242. 1911.

Müller, E., Durstfieber bei Säuglingen. Berl. klin. Wochenschr. 47, 673. 1910.

Müller, Franz, Ủber Acetonglykosurie. Archiv f. experim. Pathol. u. Pharmakol. 46, 61. 1901.

Müller, Friedrich, Handbuch der Emährungstherapie; zit. nach Langstein u. Meyer, Jahrb. f. Kinderheilk. 61, 459. 1905.

Münzer, Die harnstoffbildende Funktion der Leber. Archiv f. experim. Pathol. u. Pharmakol. 33, 164.1894.

Naunyn, Der Diabetes mellitus. 2. Aufl. Wien 1906 (Hölder).

Niemann, Die alimentäre Glykämie des Säuglings. Jahrb. f. Kinderheilk. 83, 11. 1916.

Nob é court et Marcel Maillet, Chlorurémie et Azotémie chez les nourrissons. Le Nourrisson 1, 75. 1913.

von Noorden, Die Zuokerkrankheit und ihre Behandlung. VI. Aufl. Berlin 1912 (Hirschwald). 
Novak, Leimdörfer u. Porges, Über die $\mathrm{CO}_{2}$-Spannung des Blutes in der Gravidität. Zeitschr. f. klin. Med. F5, 301. 1912.

- u. Porges, Über die Acidität des Blutes bei Osteomalazie. Wiener klin. Wochenschr. 26, 1791. 1913.

Pechstein, Reaktion des ruhenden und arbeitenden Froschmuskels. Biochem. Zeitschr. 68, 140. 1915.

Pfaundler, Über Stoffwechselstörungen bei magendarmkranken Säuglingen. Jahrb. f. Kinderheilk. 54, 247. 1901.

- Physikalisch-chemische Untersuchungen an Kinderblut. Verhandl. d. Gesellsch. f. Kinderheilk., Breslau 1904, S. 24.

- Zur Frage der ,Säurevergiftung" beim chronisch magendarmkranken Säugling. Jahrb. f. Kinderheilk. 60, 719. 1904.

- Über die aktuelle Reaktion des kindlichen Blutes. Archiv f. Kinderheilk. 41, 161. 1905 .

Porges, Über die Autointoxikation mit Säuren in der menschlichen Pathologie. Wiener klin. Wochenschr. 24, 1147. 1911.

- Leimdörfer u. Markovici, Über die $\mathrm{CO}_{2}$-Spannung des Blutes in pathologischen Zuständen. Zeitschr. f. klin. Med. 73, 389. 1910.

Reis u. Jehn, Alimentäre Galaktosurie bei Leberkrankheiten. Deutsches Arch1v f. klin. Med. 108, 187. 1912.

Rietschel, Inanition und Zuckerausscheidung im Säuglingsalter. Zeitschr. f. Kinderheilk. $\%$, 282.1913.

Rona u. Wilenko, Beiträge zur Frage der Glykolyse. IV. Biochem. Zeitschr. 62, 1. 1914.

- u. Ylppö, Der Einfluß der Acidität auf die Dissoziationskurve des Hämoglobins. Biochem. Zeitschr. (im Druck).

Rott, Die Farbreaktion des Gewebes bei der Säuglingsacidose. Monatsschr. f. Kinderheilk. \%, 73. 1908.

Roubitschek, Alimentäre Galaktosurie bei experimenteller Phosphorvergiftung. Deutsches Archiv f. klin. Med. 108, 225. 1912.

Rovere, Sulla concentrazione dell' albumina nel siero di sangue nelle gastroenteriti dei lattanti. Riv. di Clin. Pediatrica 11, 347, 1913.

Ruschhau pt, Über Acetonglykosurie. Archiv f. experim. Pathol. u. Pharmakol. 44, 127. 1900.

Salge, Der akute Dünndarmkatarrh des Säuglings. Leipzig 1906 (Thieme).

- Die Reaktion des Blutserums bei alimentärer Intoxikation des Säuglings. Vorläufige Mitteilung. Zeitschr. f. Kinderheilk. 4, 92. 1912.

- Beispiele für die Bedeutung physikalischer und physikalisch-chemischer Forschungen in der Physiologie und Pathologie des Säuglings. Zeitschr. f. Kinderheilk. $\%$, 292.1913.

Salkowski, Über die Möglichkeit der Alkalientziehung beim lebenden Thier. Arohiv f. pathol. Anat. u. Physiol. u. f. klin. Med. 58, 1. 1873.

Schlossmann u. Murschhauser, Über den EinfluB der vorangegangenen Ernährung auf den Stoffwechsel im Hunger. Biochem. Zeitschr. 53, 265. 1913.

- - Der Stoffwechsel des Säuglings in Hunger. II. Mitteil.' Biochem. Zeitschr. 58, 493. 1914. 
Schwyzer, Acidose und Anstrengung. Biochem. Zeitschr. 60, 310. 1914.

Secchi, Über die Wirkung der Salzsäure auf die Alkaliausscheidung. Biochem. Zeitschr. 6r, 143. 1914.

von Skramlik, Über Harnacidität. Zeitschr. f. physiol. Chemie r1, 290. 1911. Soldin, Zur Kenntnis der Darmfäulnis im Säuglingsalter bei verschiedenartiger Ernährung. Jahrb. f. Kinderheilk. 65, 292. 1907.

Sonnenberger, Über Intoxikationen durch Milch. Verhandl. d. Gesellsch. f. Kinderheilk., Frankfurt a. M. 1896, S. 129.

Stadelmann, Utber die Ursachen der pathologischen Ammoniakausscheidung beim Diabetes mellitus und des Coma diabeticum. Archiv f. experim. Pathol. u. Pharmakol. 17, 419. 1883.

- Über den Einfluß der Alkalien auf den menschlichen Stoffwechsel. Stuttgart 1890.

Stä ubli, Beiträge zu Pathologie und Therapie des Diabetes mellitus. Deutsohes Archiv f. klin. Med. 93, 107. 1908.

- Untersuchungen über die Acetonurie bei Diabetes mellitus. Korrespondenzbl. f. Schweizer Ärzte 38, 137. 1908.

Steinitz, Zur Kenntnis der chronischen Ernährungsstörungen der Säuglinge. I. Mitt. : Alkalistoffwechsel. Monatsschr. f. Kinderheilk. 1, 225. 1902.

- Zur Kenntnis der chroniscben Ernährungsstörungen der Säuglinge. Jahrb. f. Kinderheilk. 57, 689, 1903.

Stoeltzner, Oxypathie. Berlin 1911 (Karger).

Straub, Acidosebestimmungen bei Diabetes mellitus. Klinische Untersuchungen über die Kohlensäurespannung der Alveolarluft. Deutsches Archiv f. klin. Med: 109, 223. 1913.

- Acidose des Blutes bei Urämie. Münch. med. Wochenschr. 61, 1499. 1914.

Szili, Experimentelle Untersuchungen über Säureintoxikation. Archiv f. d. ges. Physiol. 115, 82. 1906.

Thiemich, Utber Leberdegeneration bei Gastroenteritis. Zieglers Beiträge $z$. pathol. Anat. u. z. allg. Pathol. z0, 179. 1896.

Tobler, Über die Schwefelaussoheidung im Harn der Säuglinge. Verhandl, d. Gesellsch. f. Kinderheilk., Salzburg 1909, S. 94.

- Über Veränderungen im Mineralstoffbestand des Säuglingskörpers bei akuten und chronisehen Gewichtsverlusten. Jahrb. f. Kinderheilk. 73, 566. 1911.

- u. Bessau, Allgemeine pathologische Physiologie der Ernährung und des Stoffwechsels im Kindesalter. Wiesbaden 1914 (Bergmann).

Tugendreich, Histologischer Nachweis der Acidose des Säuglings. Berl. klin. Wochenschr. 45, 886. 1908.

Valagussa, Policlinico 1902; zit. nach Heoker, Ergebnisse d. inn. Med. u. Kinderheilk. $\gamma, 260.1911$.

Walter, Untersuchungen über die Wirkung der Säuren auf den tierischen Organismus. Archiv f. experim. Pathol. u. Pharmakol. \%, 148. 1877.

Wyss, von, Über Ö̀deme durch Natrium bicarbonicum. Deutsches Archiv f. klin. Med. 111, 93. 1913. 


\section{Protokolle.}

I. Acidität des Urins bei Frühgeburten, Neugeborenen usw. bei verschiedener Ernährung.

\begin{tabular}{|c|c|c|c|c|c|c|c|}
\hline 层悹 & Datum & Name & Alter & $\begin{array}{c}\text { Ge- } \\
\text { wioht }\end{array}$ & Dlagnose & Ernährung & $\underset{\text { Urins }}{\mathbf{P}_{\mathrm{H}} \text { des }}$ \\
\hline \multirow{15}{*}{1.} & 1914 & \multirow{15}{*}{ Luste, Margot } & \multirow{6}{*}{$\begin{array}{c}24 \mathrm{Tg} . \\
29 \\
38 " \\
38 \\
6 \text { Woch. }\end{array}$} & \multirow{6}{*}{$1300 \mathrm{~g}$} & \multirow{15}{*}{$\begin{array}{l}\text { Frïhgeburt, ge- } \\
\text { sund }\end{array}$} & & \\
\hline & 21.9. & & & & & Frauenmilch & 5,61 \\
\hline & 26.9 & & & & & $n$ & 5,38 \\
\hline & 5.10. & & & & & $"$ & 5,70 \\
\hline & 5.10. & & & & & $"$ & 6,21 \\
\hline & 12.10 . & & & & & 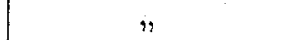 & 6,06 \\
\hline & 19. 10 . & & $7 \quad n$ & $1760 \mathrm{~g}$ & & $"$ & 5,71 \\
\hline & 26.10 . & & $8 \quad "$ & & & " & 5,79 \\
\hline & 3.11. & & $9 n$ & & & $\eta$ & 6,31 \\
\hline & 9.11. & & $10 \%$ & \multirow{3}{*}{$2200 \mathrm{~g}$} & & $n$ & 5.60 \\
\hline & 16.11. & & $11 \quad "$ & & & $n$ & 7,35 \\
\hline & 23.11 & & 12, & & & & 6,15 \\
\hline & 30.11 . & & 3 Mon. & & & $"$ & 7,01 \\
\hline & $\begin{array}{l}7.12 . \\
14.12 .\end{array}$ & & $3 \%$ Mon. & $\cdot 2450 \mathrm{~g}$ & & $n$ & $\begin{array}{l}7,74 \\
6,70\end{array}$ \\
\hline & 21.12 . & & $3 \%$ & $2680 \mathrm{~g}$ & & $"$ & $\begin{array}{l}0,10 \\
7,01\end{array}$ \\
\hline \multirow[t]{14}{*}{2.} & 18.8. & \multirow{14}{*}{$\begin{array}{c}\text { Schwersenski, } \\
\text { Hans }\end{array}$} & $21 \mathrm{Tg}$ & $1100 \mathrm{~g}$ & Fruhgeburt, ge- & Frauenmilch & 5,57 \\
\hline & 5. 9. & & 39 & $-\pi$ & sund & $n$ & 5,65 \\
\hline & $\begin{array}{l}15.9 . \\
26.9 .\end{array}$ & & $\begin{array}{l}49 \text { Mon. } \\
2 \text { Mon. }\end{array}$ & $1600 \mathrm{~g}$ & & Buttermilch $120 \mathrm{~g}$ & 5,47 \\
\hline & & & & & & Frauenmilch $200 \mathrm{~g}$ & 5,06 \\
\hline & $\begin{array}{l}5.10 . \\
12.10 .\end{array}$ & & $\begin{array}{l}21 / 4 \text { Mon. } \\
21 / 9 \quad n\end{array}$ & $2150 \mathrm{~g}$ & & 1/2 Milch" (Larosan) & $\begin{array}{l}5,24 \\
5,58\end{array}$ \\
\hline & 19.10 & & $2^{3} / 4 \quad n$ & & wind $\mathrm{sahr}$ hlan & do. & 5,12 \\
\hline & 2.11 & & 3 & & Craniotabes & Frauenmilch s. 20. X. & $\begin{array}{l}b, b 1 \\
5,21\end{array}$ \\
\hline & 9.11 . & & $3 \% \ddot{n}$ & $2250 \mathrm{~g}$ & do. & $"$ & 5,60 \\
\hline & 10.11 & & & & do. & 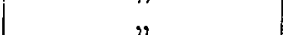 & 5,48 \\
\hline & 23.11 . & & $3^{3 / 4}$ & & do. & $n$ & 5,71 \\
\hline & 30.11. & & 4 & $2520 \mathrm{~g}$ & do. & $"$ & 6,62 \\
\hline & 7.12. & & $4^{1 / 4}$ & & do. & $n$ & 5,90 \\
\hline & 14.12. & & $41 / 2 \quad$, & & do. & 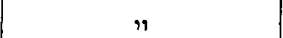 & 5,34 \\
\hline & 21.12 & & $4^{3} \% 11$ & $2740 \mathrm{~g}$ & sehr blaß, Cra- & $"$ & 5,51 \\
\hline \multirow[t]{8}{*}{ 3. } & 18. 8. & \multirow[t]{8}{*}{ Möller, Karl } & 4 Woch. & $2120 \mathrm{~g}$ & Fruhgeburt, ge- & Frauenmilch & 5,30 \\
\hline & 50 & & $11 / \mathrm{Man}$ & & sund & & \\
\hline & 5. 9. & & $13 / 2$ Mon. & $2350 \mathrm{~g}$ & & 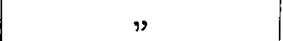 & 5,98 \\
\hline & $\begin{array}{l}20.9 \\
5.10\end{array}$ & & & $2580 \mathrm{~g}$ & & $"$ & 7,25 \\
\hline & 12.10 . & & & $\left|\begin{array}{ll}2000 & \mathrm{~g}\end{array}\right|$ & nlötalich Krïmó & & 6,91 \\
\hline & 19.10 . & & & $2840 \mathrm{~g}$ & photanc & Butermucen s. $9 . \Delta$. & $\begin{array}{l}5,88 \\
7,38\end{array}$ \\
\hline & 26.10 & & 3 Mon. & $2960 \mathrm{~g}$ & & th & 6,67 \\
\hline & 2.11. & & & $|3040 \mathrm{~g}|$ & & , & 6,39 \\
\hline
\end{tabular}


und Intoxikationsacidosis in ihren Beziehungen zueinander.

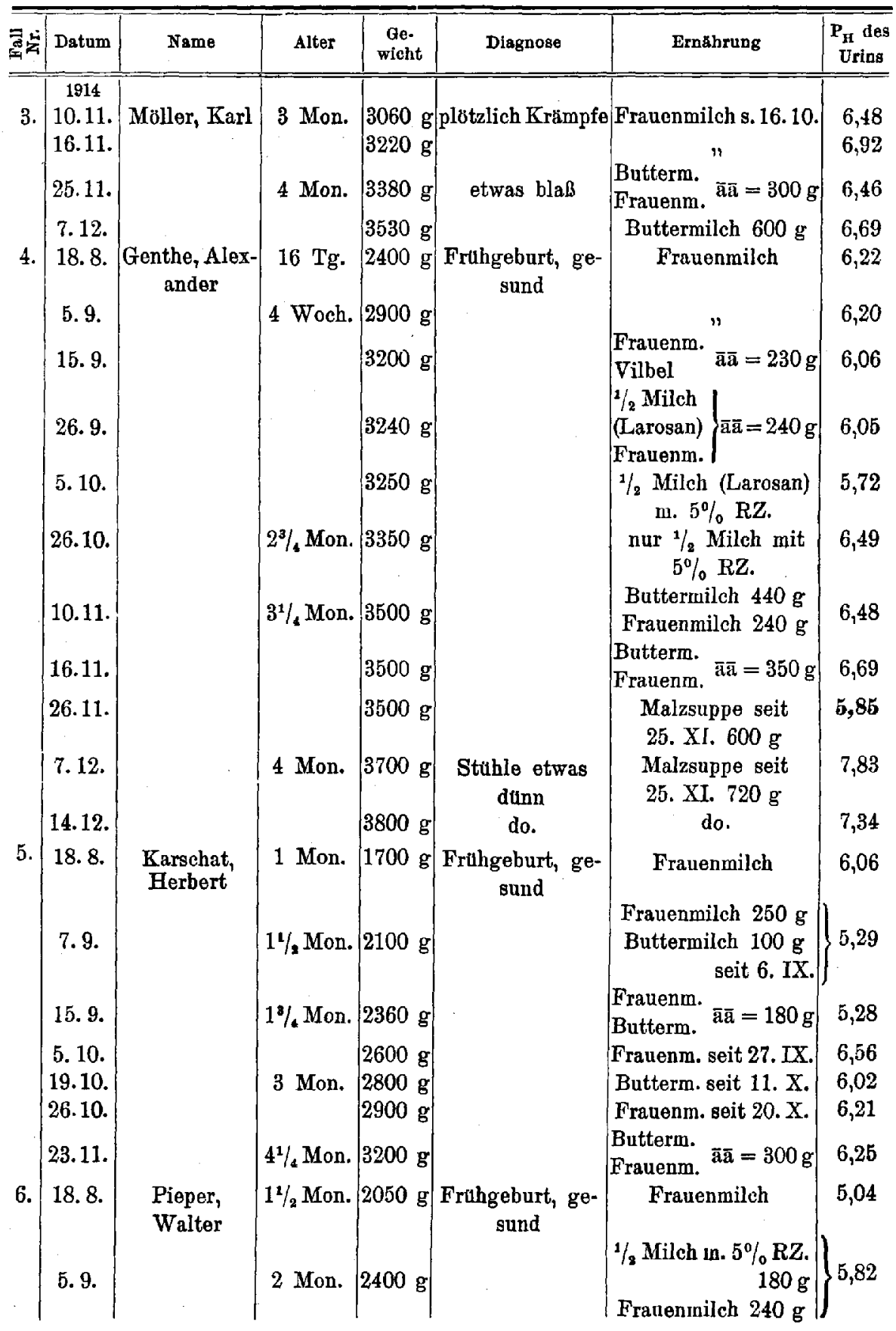


Arvo Ylppö: Neugeborenen-, Hunger-

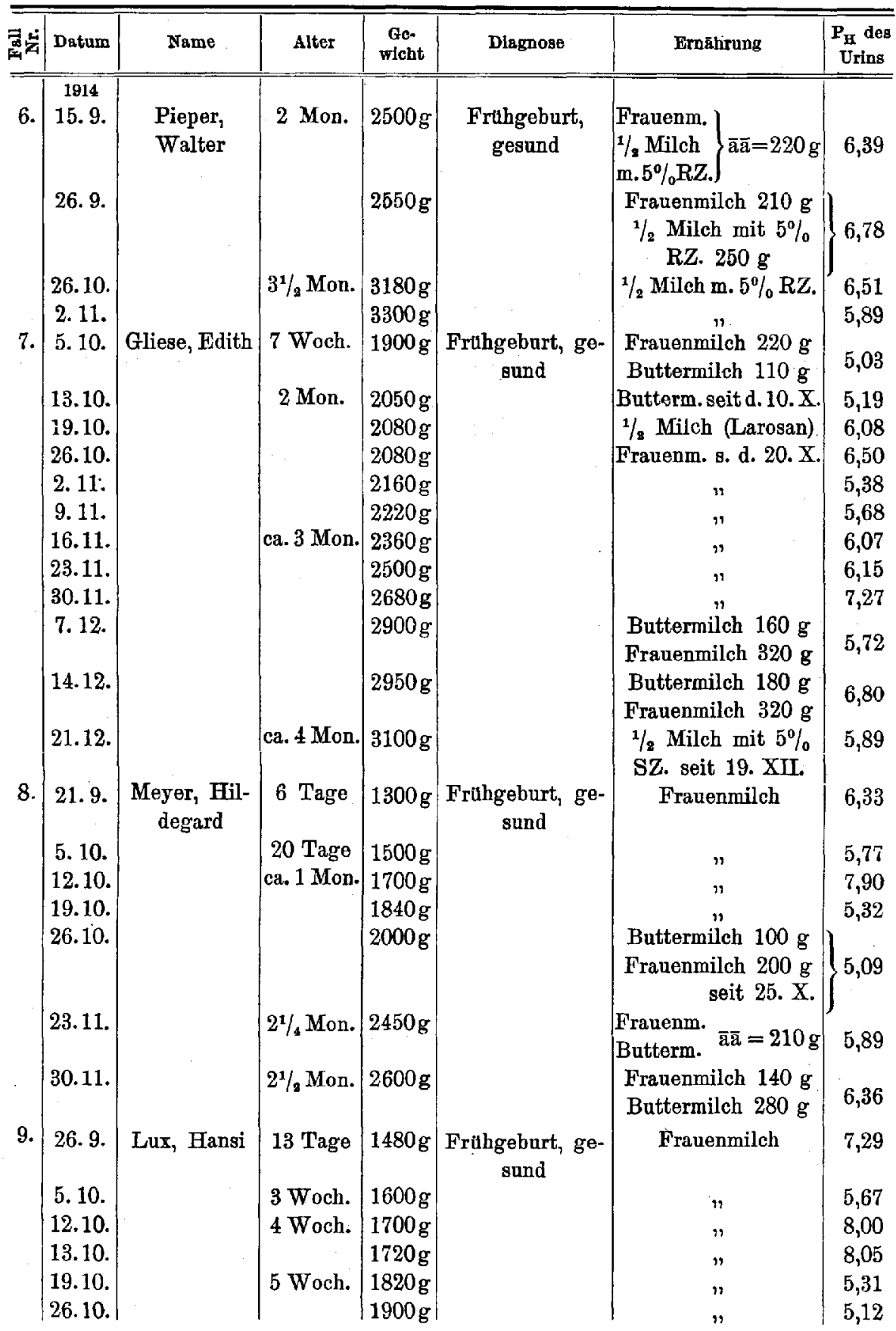


und Intoxikationsacidosis in ihren Beziehungen zueinander.

\begin{tabular}{|c|c|c|c|c|c|c|c|c|}
\hline 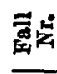 & Datum & Name & Alter & $\begin{array}{c}\text { Ge- } \\
\text { wicht }\end{array}$ & Diagnose & \multicolumn{2}{|c|}{ Ernährung } & $\mid \begin{array}{c}P_{E} \text { des } \\
\text { Urins }\end{array}$ \\
\hline & 1914 & & \multirow[b]{2}{*}{$21 / 2$ Mon. } & & & \multirow{2}{*}{\multicolumn{2}{|c|}{$\begin{array}{l}\text { Butterm. } \\
\text { Frauenm. }\end{array} \quad \overline{\mathrm{a}} \overline{\mathbf{a}}=210 \mathrm{~g}$}} & \\
\hline 9. & 23.11 & Lux, Hansi & & $2400 \mathrm{~g}$ & $\begin{array}{c}\text { Fruhgeburt, ge- } \\
\text { sund }\end{array}$ & & & 5,49 \\
\hline & 30.11 & & $2^{3} / 4$ Mon. & $2520 \mathrm{~g}$ & & \multicolumn{2}{|l|}{$"$} & 5,90 \\
\hline 10. & 8.10 & $\begin{array}{c}\text { Gozdziki, } \\
\text { Helene }\end{array}$ & 2 Tage & $1300 \mathrm{~g}$ & $\begin{array}{l}\text { Fruhgeburt mit } \\
\text { asphykt. Anfall. }\end{array}$ & \multicolumn{2}{|c|}{ Frauenmilch } & 5,63 \\
\hline & 12.10 & & 6 & $1300 \mathrm{~g}$ & do. & \multicolumn{2}{|l|}{$"$} & 8,71 \\
\hline & 13.10 & & & $1340 \mathrm{~g}$ & do. & \multicolumn{2}{|l|}{18} & 5,23 \\
\hline & 19.10 . & & & $1380 \mathrm{~g}$ & do. & \multicolumn{2}{|l|}{$n$} & 5,34 \\
\hline 11. & 18.8. & $\begin{array}{l}\text { Langfritz, } \\
\text { Hans }\end{array}$ & 6 Woch. & $2400 \mathrm{~g}$ & $\begin{array}{c}\text { Fruhgeburt, ge- } \\
\text { sund }\end{array}$ & \multicolumn{2}{|c|}{ Frauenmilch } & 7,94 \\
\hline 12. & 13.10 . & Bassewitz, L. & $11 / 2$ Mon. & $2600 \mathrm{~g}$ & Fruhgeburt, En- & \multirow{2}{*}{\multicolumn{2}{|c|}{$\begin{array}{r}\text { Frauenmilch } 370 \mathrm{~g} \\
1 / \mathrm{a} \text { Milch, 5\% RZ. } \\
160 \mathrm{~g}\end{array}$}} & 839 \\
\hline & & & & & leritis & & & 002 \\
\hline 13. & 1.10. & Bamburg & 3 Mon. & $3720 \mathrm{~g}$ & Friahgeburt & \multicolumn{2}{|c|}{ Frauenmilch $560 \mathrm{~g}$} & 6,77 \\
\hline 14. & 19.9. & Melz & 7 Tage & $1700 \mathrm{~g}$ & $\begin{array}{c}\text { Fruhgeburt, As- } \\
\text { phykt. Anfalle }\end{array}$ & \multicolumn{2}{|c|}{ Frauenmilch $250 \mathrm{~g}$} & 6,10 \\
\hline 15. & 15.9. & $\begin{array}{l}\text { Bennecke, } \\
\text { Kurt }\end{array}$ & $21 / 2$ Mon. & $2800 \mathrm{~g}$ & Fruhgeburt & \multicolumn{2}{|c|}{$\begin{array}{l}\text { Frauenm. } \\
\text { Butterm. }\end{array} \overline{\mathrm{a}} \overline{\mathrm{a}}=210 \mathrm{~g}$} & 6,84 \\
\hline \multirow[t]{9}{*}{16.} & 23.11 . & Freitag, & \multirow{3}{*}{$\begin{array}{l}\text { 12 Std. } \\
36 \text { Std. } \\
3 \text { Tage }\end{array}$} & $3000 \mathrm{~g}$ & Neugeborenes, & \multirow{2}{*}{\multicolumn{2}{|c|}{$\begin{array}{cr}\text { Frauenmulch } & 20 \mathrm{~g} \\
, & 180 \mathrm{~g}\end{array}$}} & 5,75 \\
\hline & 24.11 & Georg & & $2800 \mathrm{~g}$ & gesund & & & 5,82 \\
\hline & 25.11 & & & $2780 \mathrm{~g}$ & & $"$ & $250 \mathrm{~g}$ & 5,87 \\
\hline & 26.11 . & & $4 \quad "$ & $2840 \mathrm{~g}$ & & $"$ & $340 \mathrm{~g}$ & 5,77 \\
\hline & 28.11 & & & $2800 \mathrm{~g}$ & & $"$ & $400 \mathrm{~g}$ & 5,45 \\
\hline & 30.11 & & & $2940 \mathrm{~g}$ & & $n$ & $500 \mathrm{~g}$ & 5,84 \\
\hline & 1.12 & & & $2940 \mathrm{~g}$ & & $"$ & $500 \mathrm{~g}$ & 6,04 \\
\hline & 2.12. & & & $3000 \mathrm{~g}$ & & 1 & $520 \mathrm{~g}$ & 5,75 \\
\hline & 3.12. & & 11 & $3060 \mathrm{~g}$ & & $"$ & $450 \mathrm{~g}$ & 5,95 \\
\hline \multirow[t]{7}{*}{17.} & 20.11 & Jander, Karl & 2 & $2500 \mathrm{~g}$ & $\begin{array}{l}\text { Neugeborenes, } \\
\text { gesund (Geb.- } \\
\text { Gew. } 2700 \mathrm{~g} \text { ) }\end{array}$ & \multicolumn{2}{|c|}{ Frauenmilch $210 \mathrm{~g}$} & 5,66 \\
\hline & 29.11 & & 11 & $2560 \mathrm{~g}$ & & $n$ & $350 \mathrm{~g}$ & 5,94 \\
\hline & 30.11 & & 12 & $2600 \mathrm{~g}$ & & $"$ & $390 \mathrm{~g}$ & 7,22 \\
\hline & 1.12. & & & $2640 \mathrm{~g}$ & & $"$ & $380 \mathrm{~g}$ & 7,16 \\
\hline & 2.12 & & & $2680 \mathrm{~g}$ & & 11 & $450 \mathrm{~g}$ & 6,15 \\
\hline & 3.12 & & & $2700 \mathrm{~g}$ & & $"$ & $430 \mathrm{~g}$ & 5,86 \\
\hline & 4.12 & & 16 & $2760 \mathrm{~g}$ & & $"$ & $470 \mathrm{~g}$ & 5,64 \\
\hline 18. & 7.9. & $\begin{array}{l}\text { Zaczinski, } \\
\text { Karl }\end{array}$ & 3 & $2700 \mathrm{~g}$ & $\begin{array}{l}\text { Neugeborenes, } \\
\text { gesund }\end{array}$ & Frauenmilch & $120 \mathrm{~g}$ & 6,18 \\
\hline 19. & 17.9 & $\begin{array}{c}\text { Buschmann, } \\
\text { W. }\end{array}$ & 2 Std. & $3600 \mathrm{~g}$ & Neugeborenes & noch keine $\mathrm{Na}$ & ahrung & 6,01 \\
\hline
\end{tabular}




\begin{tabular}{|c|c|c|c|c|c|c|c|}
\hline 丞 & Datum & Name & Alter & $\begin{array}{c}\mathrm{Ge}- \\
\text { wicht }\end{array}$ & Diagnose & Ernährung & $\mid \begin{array}{c}\mathbf{P}_{\mathbf{H}} \text { des } \\
\text { Urins }\end{array}$ \\
\hline 20. & $\begin{array}{r}1914 \\
17.9\end{array}$ & Weber, $\mathbf{K}$. & 20 Tage & $3200 \mathrm{~g}$ & $\begin{array}{l}\text { Neugeborenes, } \\
\text { Gehirnblutungen }\end{array}$ & Franenmileh $500 \mathrm{~g}$ & 6,25 \\
\hline 21. & 5.10 & Schneidt, Else & 6 Mon. & $4200 \mathrm{~g}$ & $\begin{array}{l}\text { Atrophie, chron. } \\
\text { Ernahrungsstör. }\end{array}$ & $\begin{array}{c}1 / 2 \text { Milch (Larosan) } \\
5 \% \mathrm{RZ} .\end{array}$ & 4,91 \\
\hline 22. & $\begin{array}{l}5.10 \\
12.10\end{array}$ & Thiele, Käte & $71 / 2$ Mon. & $5800 \mathrm{~g}$ & $\begin{array}{c}\text { Pyelitis, Bron- } \\
\text { chitis }\end{array}$ & $\begin{array}{l}2 / 3 \text { Milch } 5 \% \text { RZ. } \\
\text { + Mondamin }\end{array}$ & $\begin{array}{l}6,70 \\
6,04\end{array}$ \\
\hline & 14.12. & & $91 / \mathrm{s}$ Mon. & $6400 \mathrm{~g}$ & & $\begin{array}{c}2 / \mathrm{s} \text { Milch } 5^{0} \% \mathrm{RZ} . \\
+ \text { Beikost }\end{array}$ & 6,43 \\
\hline 23. & 3. 9 . & $\begin{array}{l}\text { Schröder, } \\
\text { Erwin }\end{array}$ & $\begin{array}{l}1 \text { Jahr, } \\
\text { 3. Mon. }\end{array}$ & $9800 \mathrm{~g}$ & $\begin{array}{l}\text { Enterokatarrh } \\
\text { (Rekonvalesz.) }\end{array}$ & $\begin{array}{c}1 / 2 \text { Milch (Larosan) } \\
500 \mathrm{~g}\end{array}$ & 5,97 \\
\hline
\end{tabular}

Einfluß der Acidität der Nahrung aư die Acidität des Urins.

Fall Nr. 24.

Biging, Kurt, Frühgeburt, geb. 4. XII. 14 mit 1900 g Gewicht. Zartes Kind, Lunge o. B. Bekommt von Anfang an nur Frauenmilch, und zwar am 5. XII. $60 \mathrm{~g}$, am 6. XII. $140 \mathrm{~g}$, am 7. XII. $180 \mathrm{~g}$, am 8. und 9. XII. je $140 \mathrm{~g}$, dann langsam steigend bis $350 \mathrm{~g}$ am Ende des Versuches 21. XII.

Vom 12. XII. 6 Uhr früh bis 14. XII. 6 Uhr früh bekam das Kind täglich $280 \mathrm{~g}$ von folgendem saurem Nahrungsgemisch:

$$
\begin{aligned}
\frac{n-\text { Milchsäure }}{\mathrm{n} / \mathrm{I}_{2} \text {-Na-Lactat }}+4 \text { Teil } & =10 \mathrm{~cm} \\
\text { und Frauenmileh } & =100 \mathrm{~cm}
\end{aligned}
$$

$P_{H}$ dieses Gemisches war 4,58. Das Casein fiel in feinen Flocken aus. Das Kind trank die Mischung gut, nahm während des Versuches von $1720 \mathrm{~g}$ bis $1800 \mathrm{~g} \mathrm{zu}$.

vor der Säuremilch:

$$
P_{\mathbf{H}} \text { des Urins }
$$

5. XII. $8^{\mathrm{h}}$ vorm. Urin (Kind 1 Tag alt) . . . . 6,26

5. XII. 8 $8^{\text {h }}$ vorm. (Meconium $\mathrm{P}_{\mathrm{H}}=5,90$ ) . . . .

6. XII. $8^{\text {n }}$ vorm. Urin . . . . . . . . . . . 6,95

7. XII. $8^{\text {h }}$ vorm. Urin ...... . . . . 7,32

$8^{\mathrm{h}}$ nachm. Urin . . . . . . . . . 7,20

8. XII. $8^{\mathrm{h}}$ vorm. Urin . . . . . . . . . . . 6,56

9. XII. gh.vorm. Urin .......... 7,10

$7^{\text {h }}$ nachm. Urin . . . . . . . . 6,81

10. XII. gh vorm. Urin . . . . . . . . . . . 6,99

$8^{\mathrm{h}}$ nachm. Urin . . . . . . . . . 6,63

11. XII. 9h vorm. Urin . . . . . . . . . . 7,09

$6^{\text {h }}$ nachm. Urin . . . . . . . . 6,60

während der Säuremilch:

12. XII. $8^{\text {h }}$ vorm. Urin . . . . . . . . . . . 6,96

$5^{\mathrm{h}}$ nachm. Urin . . . . . . . . . . 6,59 
13. XII. 9h vorm. Polyurie . . . . . . . . 8,35

$8^{\text {h }}$ nachm. Polyurie . . . . . . 8,39

wieder nur Frauenmilch von $6^{\text {h }}$ früh

14. XII. 7h vorm. Polyurie . . . . . . . 8,53

$5^{\mathrm{h}}$ nachm. Polyurie . . . . . . . 8,02

15. XII. $9^{\mathrm{h}}$ vorm. Polyurie . . . . . . . . 8,61

$5^{\text {h }}$ nachm. Polyurie ........ . 7,70

16. XII. 9h $9^{\mathrm{h}}$ vorm. Polyurie . . . . . . . . 7,53

$8^{\text {h }}$ nachm. Polyurie . . . . . . . . 7,48

17. XII. 9h vorm. keine Polyurie mehr. . . . . 6,54

$5^{\mathrm{h}}$ naohm. Urin . . . . . . . . . 6,95

18. XII. $8^{\text {h }}$ vorm. Urin . . . . . . . . . 7,03

$6^{\text {h }}$ nachm. Urin . . . . . . . . . . 7,19

19. XII. 9 vorm. Urin . . . . . . . . . . . . 6,87

$6^{\text {h }}$ nachm. Urin . . . . . . . . . . . 6,09

20. XII. 9h vorm. Urin . . . . . . . . 7,08

$7^{\mathrm{h}}$ nachm. Urin . . . . . . . 6,58

21. XII. $8^{\mathrm{h}}$ nachm. Urin . . . . . . . . . . . 7,30

6. I. $7^{\mathrm{h}}$ vorm. Urin . . . . . . . . . . 7,20

8. I. $8^{\mathrm{h}}$ vorm. Urin . . . . . . . . . . . 7,45

10. I. $8^{\mathrm{h}}$ vorm. Urin ........... . 6,81

13. I. $8^{\mathrm{h}}$ vorm. Urin . . . . . . . . . . 6,44

25. I. $7^{\text {h }}$ vorm. Urin (Kind ca. 2 Mon. alt) . . 7,36

1. II. $8^{\mathrm{h}}$ vorm. Urin . . . . . . . . . . 6,73

15. II. $8^{\text {h }}$ vorm. Urin ... . . . . . . . . . 7,19

22. II. $8^{\mathrm{h}}$ vorm. Urin ............ 9,19

1. III. $8^{\text {h }}$ vorm. Urin (Kind ca. 4 Mon. alt, Gewicht

$2900 \mathrm{~g})$. . . . . . . 7,31

Die vorstehenden Werte sind auf Fig. 5 S. 301 graphisch dargestellt.

Fall Nr. 25.

Maudt, Else, Frühgeburt mit verschiedenen sohweren Mißbildungen. Darm gesund. Geb. 8. XI. 14 mit ca. 1600 g. Wird im Alter von 11 Tagen aufgenommen, ist dauernd cyanotisoh, kongenit. Herzfehler, deshalb Exitus 2. XII.

Bekommt täglich Frauenmilch ca. $280 \mathrm{~g}$ mit Sonde; vom 28. XI. $7 \mathrm{Uhr}$ nachmittags bis zum 30. XI. 8 Uhr vormittags bekam das Kind täglich $280 \mathrm{~g}$ von folgendem saurem Nahrungsgemisch:

$$
\begin{aligned}
\frac{\mathrm{n} \text {-Essigsäure }}{\text { n-Na-Acetat }}-\frac{1}{1} \text { Teil } & =10,0 \mathrm{~cm} \\
\text { und Frauenmilch } & =100,0 \mathrm{~cm}
\end{aligned}
$$

$P_{\mathbf{H}}$ dieses Gemisches war 4,42 (die der reinen Frauenmilch war ca. 6,80).

vor der Säuremilch:

$$
P_{H} \text { des Uring }
$$

20. XI. $8^{\mathrm{h}}$ vorm. Urin (Kind 12 Tage alt) $\ldots .6,55$

24. XI. $8^{\mathrm{h}}$ vorm. Urin ......... 7,53 
28. XI. $8^{\text {h }}$ vorm. Urin .......... . 6,63

$7^{\mathrm{h}}$ nachm. Urin . . . . . . . . . 6,74

während der Säuremilch:

29. XI. $8^{\mathrm{h}}$ vorm. Urin ........... 8,22

$8^{\text {h }}$ nachm. Urin . . . . . . . . 8,17

30. XI. $8^{\text {h }}$ vorm. Urin .......... 8,26

wieder nur Frauenmilch von $8^{\text {h }}$ früh:

30. XI. $8^{\mathrm{h}}$ nachm. Urin . . . . . . . . . 7,69

1. XII. $8^{h}$ vorm. Urin . . . . . . . . . . . 8,16

$8^{\mathrm{h}}$ nachm. Urin . . . . . . . . 8,50

2. XII. $10^{\mathrm{h}}$ vorm. Urin . . . . . . . . . . 6,32

Die Acidität der Organe und des Blutes siehe S. 392.

\section{EinfluB der Nahrung auf die Acidität des Urins.}

Fall Nr. 26.

Griebner, Hilde, Frühgeburt, geb. 11. XII. 14, 3 Mon. alt, Gewicht $3100 \mathrm{~g}$, hat bisher nur Frauenmilch bekommen. Vom 22. III. ab $1 / 2$-Milch mit $5 \%$ R.-Z. in Mengen von $480 \mathrm{~g}$ täglich bis 2 . III., von da an (6 $6^{\mathrm{h}}$ vorm.) wieder $480 \mathrm{~g}$ Frauenmilch.

Frauenmilch:

19. III. $8^{\text {h }}$ vorm. Urin . . . . . . . . . . . . 6,29

$5^{\mathrm{h}}$ nachm. Urin . . . . . . . 7,27

$1 / 2-$ Milch von heute:

22. III. $8^{\mathrm{h}}$ vorm. Urin. . . . . . . . . 6,41

Stuhl, gelblichgrün ..... . 4,93

$5^{\mathrm{h}}$ nachr. Urin. . . . . . . . 6,28

$5^{\text {h }}$ nachm. Stuhl, grün, schleimig .... 5,33

1/2-Milch:

23. III. $5^{\text {h }}$ vorm. Stuhl, gelbe Salbe . . . . 7,20

$8^{\text {h }}$ vorm. Stuhl, wie vor . . . . . . 7,4l

$8^{\mathrm{h}}$ vorm. Urin. . . . . . . . . 6,52

$5^{\text {h }}$ nachm. Urin. . . . . . . . . . 6,64

27. III. $8^{\mathrm{h}}$ vorm. Urin. . . . . . . . . 6,07

$5^{\text {h }}$ nachm. Urin. . . . . . . . 6,05

28. III. $8^{\text {h }}$ vorm. Stuhl, weißlichgelbe Paste : . 6,65

29. III. $8^{\text {h }}$ vorm. Stuhl, wie vor . . . . . . 6,15

1. IV. $8^{\text {h }}$ vorm. Urin. : . . . . . . . . , 5,87

$5^{\text {h }}$ nachm. Urin. . . . . . . . . 5,62

$5^{\text {h }}$ nachm. Stuhl, gelblichgrünliche Salbe $.6,26$

$480 \mathrm{~g}$ Frauenmileh von heute $6^{\mathrm{h}}$ vorm.:

Frauenmilch:

2. IV. $8^{\mathrm{h}}$ vorm. Urin. . . . . . . . . . 5,78

$6^{\mathrm{h}}$ nachm. Stuhl, gelblich, salbig . . . . 5,43

5. IV. $8^{\mathrm{h}}$ vorm. Urin. . . . . . . . . . 6,25

$1 \mathrm{I}^{\mathrm{h}}$ vorm. Stuhl, wie vor . . . . . . 5,10

Die gefundenen Werte sind auf Fig. 3 graphisch dargestelit. 
und Intoxikationsacidosis in ihren Beziehungen zueinander.

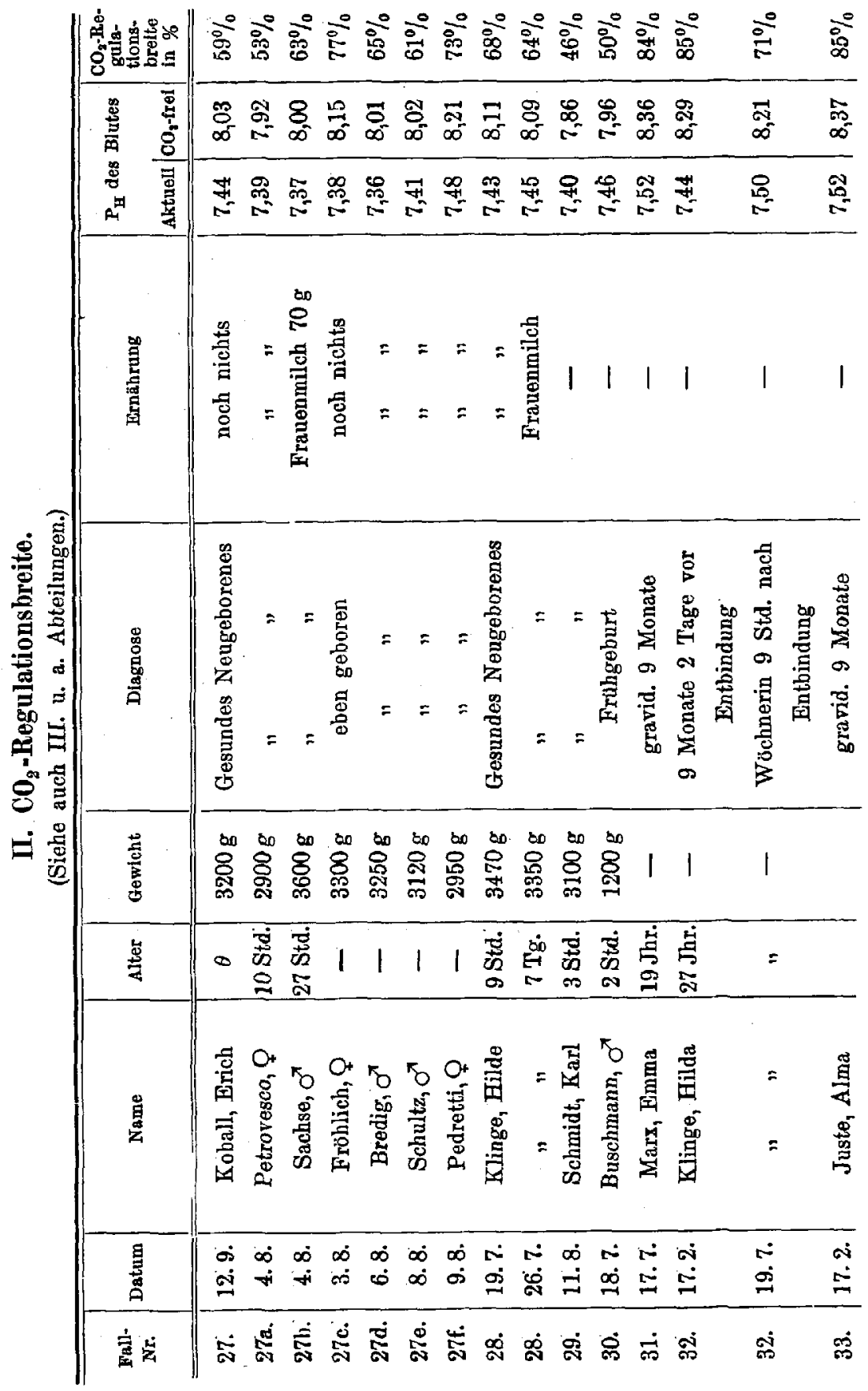




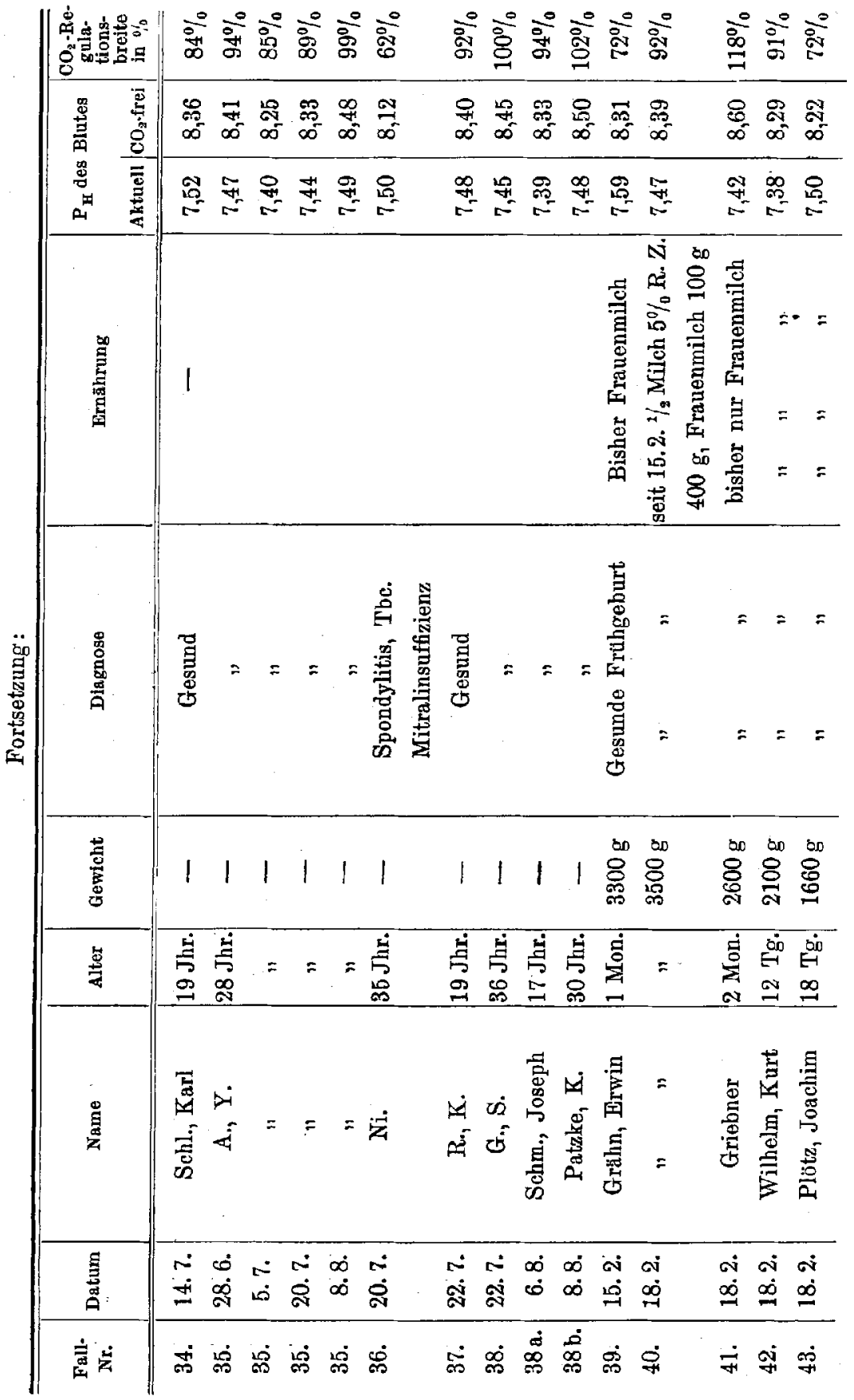


und Intoxikationsacidosis in ihren Beziehungen zueinander.

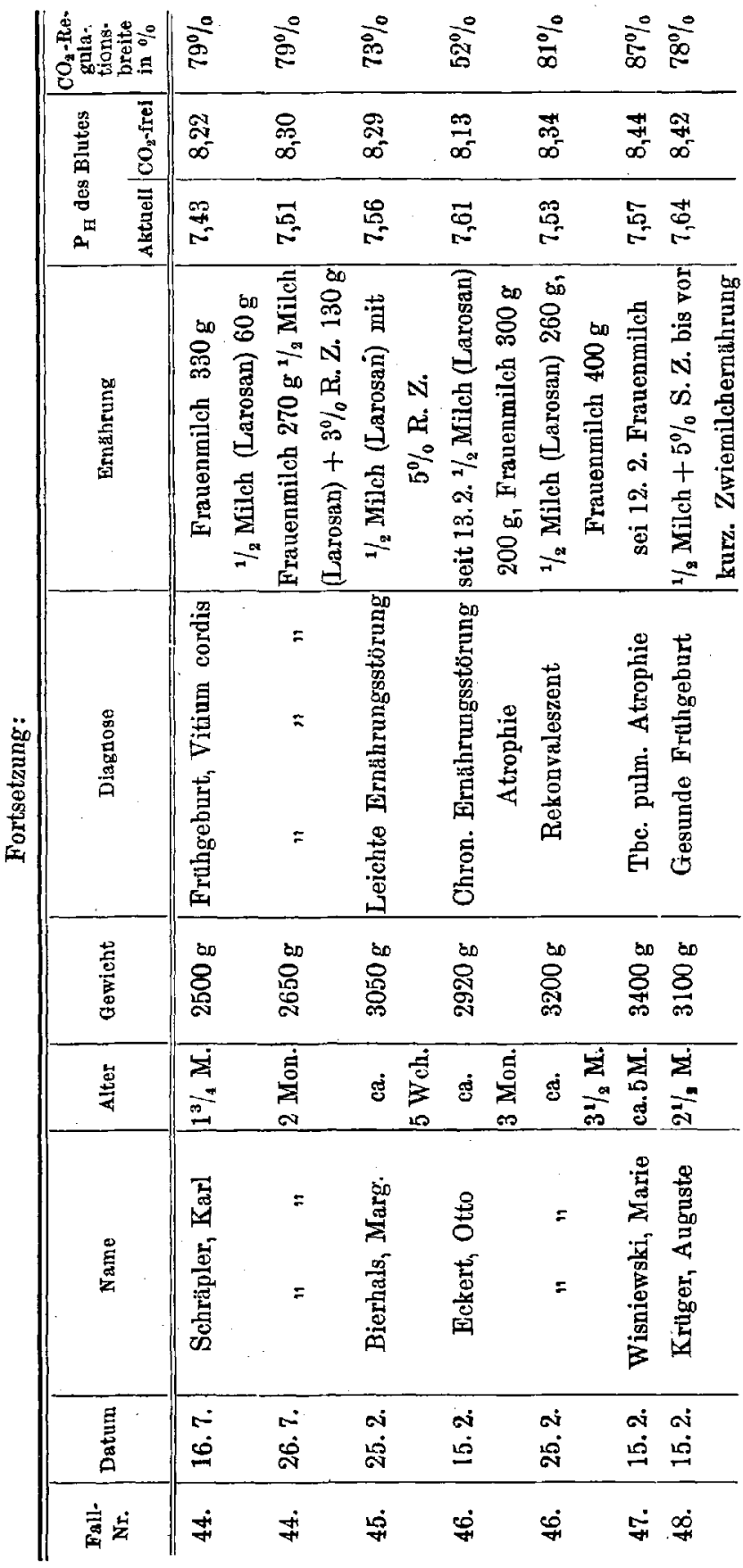

Zeitschrift für Kinderheilkunde. 


\section{III. $\mathbf{O}_{2}$-Dissoziationskurve des Blutes mit zunehmendem Alter.}

Fall Nr. 49. (Siehe auch Fall Nr. 60.)

Verschiebungen im Verlaufe der $\mathrm{O}_{2}$-Dissoziationskurve des Hämoglobins mit zunehmendem Alter.

Kind Jacoby, Hubert; klinische Notizen siehe S. 398.

$\mathrm{O}_{2}$-Dissoziationskurve des Hämoglobins.

Punkt I.

3,0 ccm Nabelschnurblut im Tonometer, daselbst ununterbrochen $25 \mathrm{Min}$. geschüttelt.

$\left.\begin{array}{llll}\text { Temp. } & 20^{\circ} & \text { Gasanalysen } & \mathrm{I}=3,50 \\ \text { Druck } & \mathbf{7 5 2} \mathrm{mm} \mathrm{Hg} & \text { aus dem Tonometer } & \mathrm{II}=3,45\end{array}\right\}=3,5 \% \mathrm{O}_{2}$

A. Differentialmanometer I bei $19^{\circ} \mathrm{im}$ Wasserbade.

In die Birne ca. 0,1 ccm Blut.

\begin{tabular}{|c|c|c|}
\hline $\begin{array}{c}\text { In die linke Birne } \\
\text { das gesättigte Blut } \\
7,72\end{array}$ & $\begin{array}{c}\text { In die rechte Birne das } \\
\text { zu untersuch. Blut } \\
7,67\end{array}$ & Differenz \\
\hline 7,10 & 8,22 & 1,17 \\
\hline 9,67 & 5,76 & 3,86 \\
\hline
\end{tabular}

Gleichgewicht bei .....

Nach dem Schütteln ... .

Kaliumferricyanid rechts,

$\begin{array}{ccc}\text { Hähne vorher geöffnet } \cdot . \quad & 9,67 & 5,76 \\ \text { B. Differentialmanometer II bei } 19^{\circ} & \end{array}$

In die Birne ca. $0,1 \mathrm{ccm}$ Blut.

Gleichgewicht bei In die linke Birne In die rechte Birne das

Nach dem Schütteln . . . $\quad 6,86 \quad 8,10 \quad 1,26$

Kaliumferricyanid rechts,

Hähne vorher geöffnet . . $\quad 9,50 \quad 5,40 \quad 4,08$

$$
\begin{aligned}
& \text { \% Sättigung } \left.\begin{array}{l}
\mathrm{A}=69,6 \\
\mathrm{~B}=69,1
\end{array}\right\}=69,4 \% \quad \mathrm{O}_{2} \\
& \mathrm{O}_{2} \text { Druck . . . . . }=26,3 \mathrm{~mm} \mathrm{Hg} \\
& \mathbf{P}_{\mathrm{H}} \ldots \text {. . . . . . }=7,75
\end{aligned}
$$

Punkt II.

$3,0 \mathrm{ccm}$ Nabelschnurblut im Tonometer, daselbst ununterbrochen $25 \mathrm{Min}$. bei $38^{\circ}$ geschüttelt.

Temp. $20^{\circ}$

Druck $752 \mathrm{~mm} \mathrm{Hg}$

$\left.\begin{array}{ll}\text { Gasanalysen } & \mathrm{I}=\mathbf{5 , 0} \\ \text { aus dem Tonometer } & \mathrm{II}=4,7\end{array}\right\}=\mathbf{4 , 8 5} \% \mathrm{O}_{2}$

A. Differentialmanometer I bei $19^{\circ} \mathrm{im}$ Wasserbade.

In die Birne ca. $0,1 \mathrm{ccm}$ Blut.

Gleichgewicht ber......

$\begin{array}{ccc}\text { In die linke Birne } & \text { In die rechte Birne das } \\ \text { das gesättigte Blut } & \text { zu untersuch. Blut } & \text { Differenz }\end{array}$

Nach dem Schütteln . . . . $\quad 7,30$

7,67

8,10

0,85

Kaliumferricyanid rechts,

Hähne vorher geöffnet

9,70

5,70

3,95 
B. Differentialmanometer II bei $19^{\circ}$ im Wasserbade.

In die Birne ca. 0,1 com Blut.

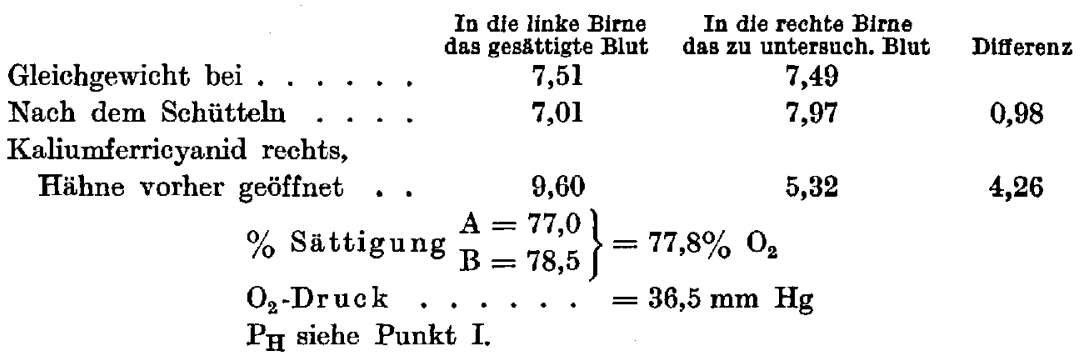

Mit Hilfe der obigen Zahlen wurden die Werte $\mathbf{n}$ und $\mathrm{K}$ in der Gleichgewichtsformel bereehnet:

$$
\begin{aligned}
\mathbf{K} & =0,0119 \\
\mathbf{n} & =1,63
\end{aligned}
$$

und danach durch dieselbe Formel ein dritter Punkt auf der Dissoziationskurve rechnerisch bestimmt, und zwar:

$$
\begin{aligned}
\text { Punkt III. } & \\
\% \text { Sättigung } & =90,3 \% \mathrm{O}_{2} \\
\mathrm{O}_{2} \text {-Druck } & =60,0 \mathrm{~mm} \mathrm{Hg}
\end{aligned}
$$

Diese drei Punkte sind auf der Fig. 9 I zu einer Dissoziationskurve vereinigt. Hierauf sind die Punkte I und II mit $\bullet$, Punkt III mit * gezeichnet.

$\mathrm{O}_{2}$-Dissoziationskurve des Blutes beim Kind Jacoby, 5 Tage alt. Punkt I.

2,0 ccm Blut im Tonometer, daselbst ununterbrochen $25 \mathrm{Min}$. bei $30^{\circ}$ geschüttelt.

$$
\left.\begin{array}{llll}
\text { Temp. } 17^{\circ} & \text { Gasanalysen } & \mathrm{I}=2,6 \\
\text { Druck } & 750 \mathrm{~mm} \mathrm{Hg} & \text { aus dem Tonometer } & \mathrm{II}=2,6
\end{array}\right\}=2,6 \% \mathrm{O}_{2}
$$

A. Differentialmanometer I bei $16^{\circ} \mathrm{im}$ Wasserbade.

In die Birne ca. $0,1 \mathrm{~cm}$ Blut.

$\begin{array}{lcccc} & & \begin{array}{c}\text { In die linke Birne } \\ \text { das gesattigte Blut }\end{array} & \begin{array}{c}\text { In die rechte Blrne das } \\ \text { zu untersuch. Blut }\end{array} & \text { Dlfferenz } \\ \text { Gleichgewicht bei . . . . . } & 7,65 & 7,60 & \\ \text { Nach dem Schütteln } & \text {. . . } & 8,60 & 6,60 & 1,95 \\ \begin{array}{l}\text { Kaliumferricyanid rechts, } \\ \text { Häbne vorher nicht geöffnet }\end{array} & 6,42 & 8,70 & 2,33\end{array}$

B. Differentialmanometer II bei $16^{\circ}$ im Wasserbade.

In die Birne ca. $0,1 \mathrm{ccm}$ Blut.

$\begin{array}{ccc}\begin{array}{c}\text { In die linke Birne } \\ \text { das gesättigte Blut }\end{array} & \begin{array}{c}\text { In die rechte Birne das } \\ \text { zu untersuch. Blut }\end{array} & \text { Differenz } \\ 7,40 & 7,40 & \\ 6,13 & 8,54 & 2,41 \\ & & \\ 8,85 & 5,72 & 3,13\end{array}$

Gleichgewicht bei . . . . . .

Hähne vorher nicht geöffnet

$$
\left.\% \text { Sättigung } \begin{array}{l}
\mathrm{A}=54,4 \\
\mathrm{~B}=56,5
\end{array}\right\}=55,5 \% \mathrm{O}_{2}
$$




$$
\begin{aligned}
& \mathrm{O}_{2} \text {-Druck . . . . }=19,5 \mathrm{~mm} \mathrm{Hg} \\
& \mathrm{P}_{\mathrm{H}} \ldots \ldots \ldots . \ldots=7,82 \\
& \mathrm{O}_{2} \text {-Dissoziationskurve des Hämoglobins. } \\
& \text { Punkt II. }
\end{aligned}
$$

2,0 com Blut im Tonometer, daselbst ununterbrochen 25 Min. bei $38^{\circ}$ geschüttelt.

\begin{tabular}{|c|c|c|c|}
\hline$t$ & $\begin{array}{l}\text { In die linke Birne } \\
\text { das gesättigte Blut }\end{array}$ & $\begin{array}{l}\text { In die rechte Birne das } \\
\text { zul untersuch. Blut }\end{array}$ & Differenz \\
\hline teln & & 711 & 0.92 \\
\hline chts, & & & \\
\hline Hähne vorher nicht geöffnet & 5,70 & 9,43 & 3,78 \\
\hline
\end{tabular}

$$
\left.\begin{array}{lll}
\text { Temp. } 17^{\circ} & \text { Gasanalysen } & \mathrm{I}=4,76 \\
\text { Druck } & 750 \mathrm{~mm} \mathrm{Hg} & \text { aus dem Tonometer } \\
\mathrm{II}=4,48
\end{array}\right\} 4,6 \% \mathrm{O}_{2}
$$

\begin{tabular}{|c|c|c|c|}
\hline Gleichgewicht bei & $\begin{array}{r}\text { In die linke Birne } \\
\text { das gesättigte Blut } \\
7,40\end{array}$ & $\begin{array}{c}\text { In die rechte Birne das } \\
\text { zu untersuch. Blut } \\
7,40\end{array}$ & Differenz \\
\hline Nach dem Schütteln & 6,88 & 7,82 & 0,94 \\
\hline \multicolumn{4}{|l|}{ Kaliumferrioyanid rechts, } \\
\hline Hähne vorher nicht geöffnet & 9,40 & 5,20 & 4,20 \\
\hline $\begin{array}{l}\text { \% Sättigung } \\
\mathrm{O}_{2}-\text { Druck } \\
\mathrm{P}_{\mathrm{H}} \text { siehe oben. }\end{array}$ & $\begin{array}{l}\left.\begin{array}{l}A=80,0 \\
B=81,7\end{array}\right\}= \\
\therefore .\end{array}$ & $\begin{array}{l}9 \% \mathrm{O}_{2} \\
5 \mathrm{~mm} \mathrm{Hg}\end{array}$ & \\
\hline
\end{tabular}

A. Differentialmanometer $I$ bei $16^{\circ}$ im Wasserbade.

In die Birne ca. $0,1 \mathrm{ccm}$ Blut.

B. Differentialmanometer II bei $16^{\circ}$ im Wasserbade.

In die Birne 0,1 com Blut.

Mit Hilfe der obigen Zahlen wurden die Werte $\mathrm{n}$ und $\mathrm{K}$ in der Gleichgewichtsformel berechnet:

$$
\begin{aligned}
\mathrm{K} & =\mathbf{0 , 0 0 1 2 4} \\
\mathrm{n} & =\mathbf{2 , 3} .
\end{aligned}
$$

Diese Punkte sind zu einer Dissoziationskurve auf der Fig. 9 II vereinigt.

$\mathrm{O}_{2}$ - Dissoziationskurve des Blutes vom Kind Jacoby, 2 Monate alt. Punkt I.

2,0 ccm Blut im Tonometer, daselbst ununterbrochen $25 \mathrm{Min}$. bei $38^{\circ}$ geschüttelt.

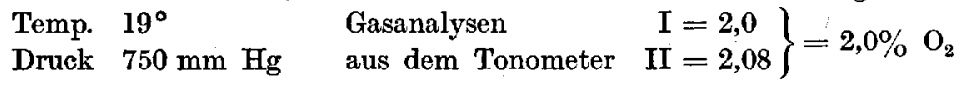

A. Differentialmanometer I bei $17^{\circ}$ im Wasserbade.

In die Birne ca. 0,1 com Blut.

$\begin{array}{ccc}\begin{array}{c}\text { In die rechte Birne } \\ \text { das gesättigte Blut }\end{array} & \begin{array}{c}\text { In die linke Birne das } \\ \text { zu untersuch. Blut }\end{array} & \text { Differenz } \\ \mathbf{7 , 6 0} & 7,65 & \\ 6,90 & 8,30 & 1,35 \\ & & \\ 8,20 & 6,99 & 1,26\end{array}$

Gleichgewicht bei . . . . . .

Nach dem Schütteln ... . 
B. Differentjalmanometer II bei $17^{\circ}$ im Wasserbade.

In die Birne ca. 0,1 ccm Blut.

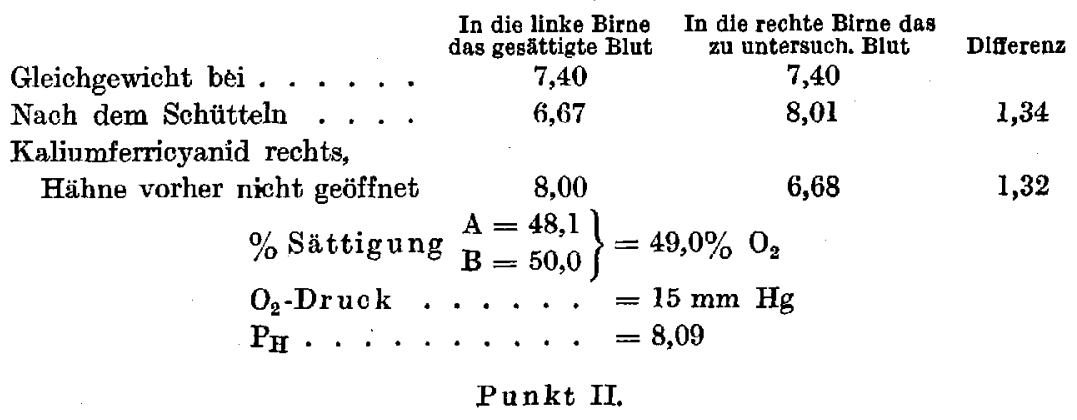

2,0 ccm Blut im Tonometer, daselbst ununterbrochen 25 Min. bei $38^{\circ}$ geschüttelt.
Temp. $18^{\circ}$
Drúck $750 \mathrm{~mm} \mathrm{Hg}$
Gasanalysen
$\left.\begin{array}{r}\mathrm{I}=4,0 \\ \mathrm{II}=4, \mathrm{I}\end{array}\right\}=4,05 \% \mathrm{O}_{2}$

A. Differentialmanometer I bei $17^{\circ}$ im Wasserbade.

In die Birne ca. $0,1 \mathrm{ccm}$ Blut.

$\begin{array}{lcccc} & & \begin{array}{c}\text { In die rechte Birne } \\ \text { das gestitigte Blut }\end{array} & \begin{array}{c}\text { In die linke Birne das } \\ \text { zut untersuch. Blut }\end{array} & \text { Differenz } \\ \text { Gleichgewicht bei . . . . . . } & 7,60 & 7,63 & \\ \text { Nach dem Schütteln . . . . } & 7,46 & 7,75 & 0,26 \\ \text { Kaliumferricyanid links, } & & & \\ \text { Hähne vorher nicht geöffnet } & 8,69 & 6,51 & \mathbf{2 , 2 1}\end{array}$

B. Differentialmanometer II bei $17^{\circ}$ im Wasserbade.

In die Birne ca. $0,1 \mathrm{~cm}$ Blut.

Gleichgewicht bei ... . .

$\begin{array}{ccc}\begin{array}{c}\text { In die linke Birne } \\ \text { das gesättigte Blut }\end{array} & \begin{array}{c}\text { In die rechte Birne das } \\ \text { zu untersuch. Blut }\end{array} & \text { Differenz } \\ 7,40 & 7,40 & \\ 7,17 & 7,50 & 0,33\end{array}$

Kaliumferricyanid rechts,

Hähne vorher nicht geöffnet

$$
\begin{aligned}
& \text { \% Sättigung } \left.\begin{array}{l}
\mathrm{A}=89,5 \\
\mathrm{~B}=88,5
\end{array}\right\}=89 \% \mathrm{O}_{2} \\
& \mathrm{O}_{2} \text {-Druck . . . . . }=30 \mathrm{~mm} \mathrm{Hg} \\
& \mathrm{P}_{\mathrm{H}} \text { wie vor. }
\end{aligned}
$$

Diese Werte sind auf der Fig. 9, III zu einer Dissoziationskurve vereinigt.

Fall Nr. 50.

$\mathrm{O}_{2}$-Dissoziationskurve des Hämoglobins im Nabelschnurblut.

11. VIII. Gesundes Neugeborenes.

Punkt I.

$3,0 \mathrm{ccm}$ Nabelschnurblut im Tonometer, daselbst ununterbrochen $25 \mathrm{Min}$. bei $38^{\circ}$ geschüttelt.

Temp.: $22^{\circ}$

Druck: $753 \mathrm{~mm} \mathrm{Hg}$

$\left.\begin{array}{lr}\text { Gasanalysen } & \mathrm{I}=3,5 \\ \text { aus dem Tonometer } & \text { II }=3,1\end{array}\right\}=3,3 \% \quad \mathrm{O}_{2}$ 
A. Differentialmanometer I bei $22^{\circ}$ im Wasserbade.

In die Birne ca. $0,1 \mathrm{ccm}$ Blut.

\begin{tabular}{|c|c|c|c|}
\hline Gleichgewicht bei & $\begin{array}{c}\text { In die linke Birne } \\
\text { das gesāttigte Blut } \\
7,74\end{array}$ & $\begin{array}{c}\text { In die rechte Birne das } \\
\text { zu untersuch. Blut } \\
7,69\end{array}$ & Differenz \\
\hline Nach dem Schütteln & 7,18 & 8,23 & 1,10 \\
\hline $\begin{array}{c}\text { Kaliumferricyanid rechts, } \\
\text { Hähne vorher geöffnet }\end{array}$ & 9,43 & 6,10 & 3,28 \\
\hline
\end{tabular}

B. Differentialmanometer II bei $22^{\circ}$ im Wasserbade.

In die Birne ca. $0,1 \mathrm{com}$ Blut.

\begin{tabular}{|c|c|c|c|c|c|}
\hline & & $\begin{array}{l}\text { In die linke } \mathbf{B} \\
\text { das gesättigte }\end{array}$ & & $\begin{array}{l}\text { In die rechte Birne das } \\
\text { zu untersuch. Blut }\end{array}$ & Differenz \\
\hline Gleichgewicht bei & . & 7,49 & & 7,49 & \\
\hline Nach dem Schütteln & . & 6,90 & & $-8,09$ & 1,19 \\
\hline aliumferricyanid rechts, & & & & & \\
\hline Hähne vorher geöffnet & & 8,93 & & 6,02 & 2,91 \\
\hline $\begin{array}{l}\mathrm{O}_{2} \text {-Druck } \\
\mathbf{P}_{\mathrm{H}} \text { (des } \mathrm{CO}_{2} \text {-freie } \\
\mathbf{P}_{\mathbf{H}} \text { (des frischen, }\end{array}$ & n B & $\begin{array}{l}\text { Sättig ung } \\
\cdot \cdot \cdot \cdot \cdot \\
\text { tes aus der } \\
\text { haltigen Blu }\end{array}$ & $\begin{array}{l}\mathrm{A} \\
\mathrm{B}\end{array}$ & 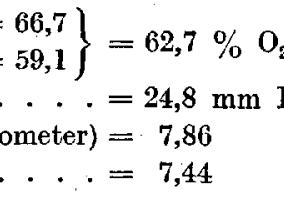 & [g \\
\hline
\end{tabular}

Dieser Punkt fällt genan in die Dissoziationskurve des Nabelschnurblutes von Kind Jacoby auf Fig. 9, I.

Fall Nr. 51.

$\mathrm{O}_{2}$-Dissoziationskurve des Hämoglobins im Nabelschnurblut.

7. VIII. Gesundes Neugeborenes.

\section{Punkt I.}

$3,0 \mathrm{ccm}$ Nabelschnurblut im Tonometer, daselbst ununterbrochen $25 \mathrm{Min}$. bei $38^{\circ}$ geschüttelt.

Temp. : $20^{\circ}$ Gasgemisch . . . . . . $=3,6 \% \mathrm{O}_{2}$

Druck: $750 \mathrm{~mm} \mathrm{Hg}$

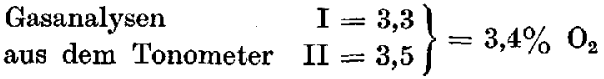

Differentialmanometer I bei $19,5^{\circ} \mathrm{im}$ Wasserbade.

In die Birne ca. $0,1 \mathrm{ccm}$ Blut.

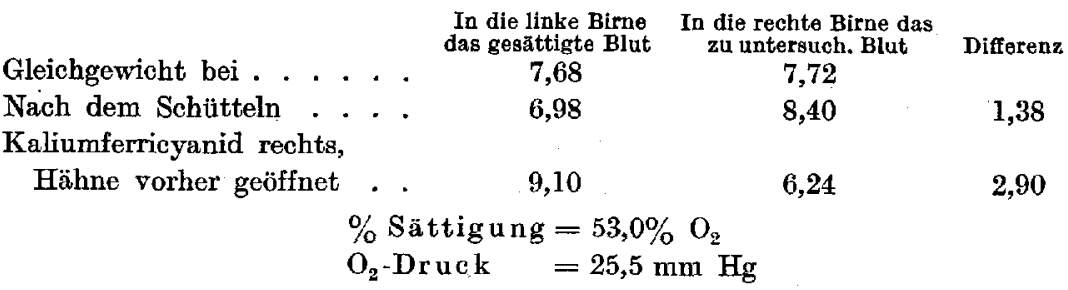

Dieser Punkt gehört zu einer Dissoziationskurve, die einen noch niedrigeren Verlauf nimmt als die des Nabelschnurblutes von Kind Jacoby auf Fig. 9, I. 
Fall Nr. 52.

$\mathrm{O}_{2}$-Dissoziationskurve des Hämoglobins im Schwangerenblut.

9. IX. 14. Hildegard Jacoby, 21 Jahre alt, I Woche vor Entbindung Blutentnabme.

\section{Punkt I.}

$3,0 \mathrm{ccm}$ Schwangerenblut im Tonometer, daselbst ununterbroohen $25 \mathrm{Min}$. bei $38^{\circ}$ geschüttelt.

Temp.: $21^{\circ}$

Druck: $746 \mathrm{~mm} \mathrm{Hg}$

Gasanalysen

aus dem Tonometer $\mathrm{II}=3,00\}=3,1 \% \mathrm{O}_{2}$

Differentialmanometer I bei $20^{\circ} \mathrm{im}$ Wasserbade.

In die Birne ca. $0,1 \mathrm{ccm}$ Blut.

$\begin{array}{ccc}\begin{array}{c}\text { In die linke Birne } \\ \text { das gesattigte Blut }\end{array} & \begin{array}{c}\text { In dfe rechte Birne das } \\ \text { zu untersuch. Blut }\end{array} & \text { Dlfferenz } \\ \mathbf{7 , 4 0} & 7,40 & \\ 6,97 & 7,69 & 0,72\end{array}$

Gleichgewicht bei . . . . .

6,97

Kaliumferricyanid rechts,

Hähne vorher nicht geöffnet

$10,42 \quad 8,20$

$$
\begin{gathered}
\% \text { Sättigung }=75,5 \% \mathrm{O}_{2} \\
\mathrm{O}_{2} \text {-Druck }=23,13 \mathrm{~mm} \mathrm{Hg} \\
\mathrm{P}_{\mathrm{H}} \cdot \cdot \cdot \cdot=7,95 \\
\text { Punkt II. }
\end{gathered}
$$

$3,0 \mathrm{cem}$ Schwangerenblut im Tonometer, daselbst ununterbrochen $25 \mathrm{Min}$. bei $38^{\circ}$ geschüttelt.
Temp.: $21^{\circ}$
Gasanalysen
Druck: $746 \mathrm{~mm} \mathrm{Hg}$

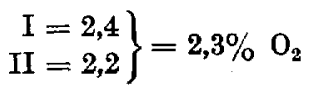

A. Differentialmanometer I bèi $18^{\circ} \mathrm{im}$ Wasserbade.

In die Birne ca. $0,1 \mathrm{ccm}$ Blut.

In die linke Birne

In die linke Birne In die rechte Birne das

Gleichgewicht bei . . . . . . $\quad 8,62$

$\begin{array}{lcr}8,62 & \text { zu untersuch. Blut } & \text { Differenz } \\ 7,42 & 8,60 & \\ & 9,00 & 1,60\end{array}$

Kaliumferrioyanid rechts,

Hähne vorher nicht geöffnet

$$
9,32
$$

\begin{tabular}{|c|c|c|c|}
\hline Gleichgewicht bei & $\begin{array}{c}\text { In die linke Birne } \\
\text { das gesatttigte Blut } \\
8,04\end{array}$ & $\begin{array}{c}\text { In die rechte Birne das } \\
\text { zu untersuch. Blut } \\
\mathbf{8 , 0 0}\end{array}$ & Differenz \\
\hline Nach dem Sohütteln & 8,68 & 9,97 & 1,33 \\
\hline \multirow{2}{*}{\multicolumn{4}{|c|}{$\begin{array}{l}\text { Kaliumferrioyanid rechts, } \\
\text { Hähne vorher nicht geöffnet }\end{array}$}} \\
\hline & 10,14 & 8,50 & 1,60 \\
\hline $\begin{array}{l}\% \text { Sättigung } \\
\mathrm{O}_{2} \cdot \text { Druck } \\
\mathrm{P}_{\mathrm{H}} \text { wie vor. }\end{array}$ & $\begin{array}{l}\left.\begin{array}{l}A=56,7 \\
B=54,6\end{array}\right\}= \\
\therefore .\end{array}$ & $\begin{array}{l}, 7 \% \mathrm{O}_{2} \\
, 16 \mathrm{~mm} \mathrm{Hg}\end{array}$ & \\
\hline
\end{tabular}

7,20

B. Differentialmanometer II bei $18^{\circ}$ im Wasserbade.

In die Birne ca, 0,1 ccm Blut.

Diese Punkte sind auf Fig. 9 S. 306 mit $\Delta$ gezeiohnet. Sie fallen beinahe in die Kurve des Blutes rom Kind Jacoby im Alter von 2 Mon. 


\title{
IV. Die Reaktion des Blutes und der Gewebe (einschl. einiger Disso- ziationskurven) bei Frühgeburten.
}

\author{
Fall Nr. 53.
}

Harms, Wilhelm, 2 Wochen alt, Erysipel, am 13. IX. 14 einige Wochen zu früh geboren, von Anfang an künstlich ernährt, soll bis zum 27. IX. gesund gewesen sein. Dann plötzliche Schwellung der rechten Gesichtshälfte, hohes Fieber. Am folgenden Tage, 28. IX., zur Aufnahme gebracht. Status: kleines, leidlich ernährtes Kind, $2660 \mathrm{~g}$; starke Schwellungen und harte Infiltrationen der ganzen rechten Gesichtshälfte und der rechten Halsseite, Untertemperatur. Sieht elend und blaB aus, bekommt Frauenmilch und $1 / 2$ Milch mit Sonde, Stühle etwas zer. fahren. In der Nacht zum 29. IX. plötzlich stark erschwertes Atmen, der Hals stark geschwollen, um $6^{\mathrm{h}} 30^{\prime}$ unter zunehmender Atemnot Exitus.

Herzpunktion und Sektion gleich danach.

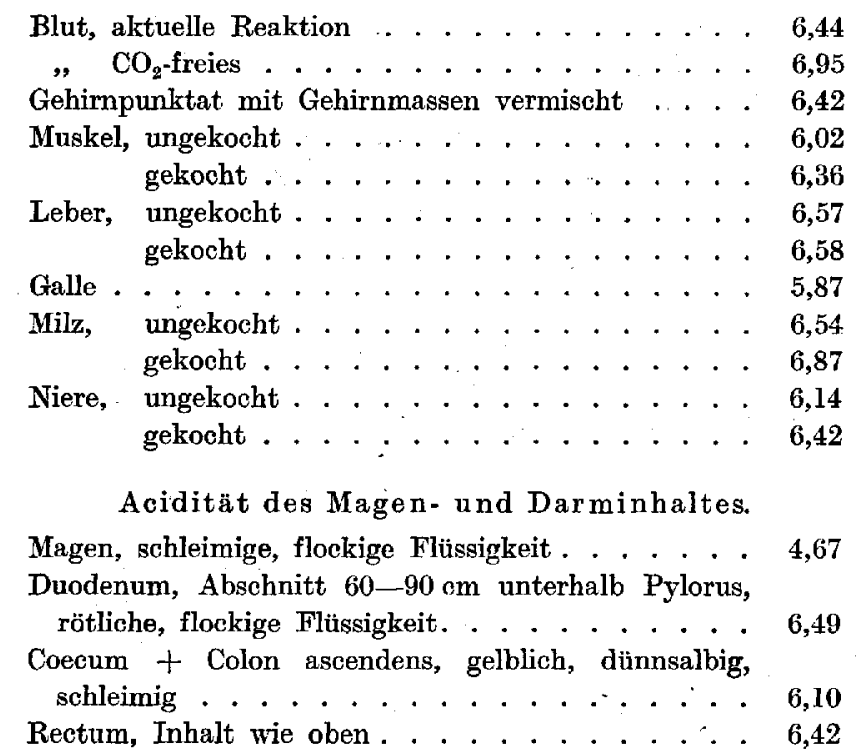

Die Sektion ergab phlegmonöses Erysipel des Gesichts mit anschließendem Lary nxödem.

Die vorstehenden Zahlen sind auf der Kurve 11, II graphisch dargestellt. Außerdem wurde noch bestimmt:

die Dissoziationskurve des $\mathrm{O}_{2}$-Hä moglobins.

Punkt I.

2,0 ccm Herzblut im Tonometer, daselbst ununterbrochen $25 \mathrm{Min}$. geschüttelt

Temp.: $20^{\circ}$

Druck: $752 \mathrm{~mm} \mathrm{Hg}$

$$
\begin{aligned}
& \text { Gasgemisch ....... . }=2,0 \% \mathrm{O}_{2} \\
& \text { Gasanalyse } \\
& \text { aus dem Tonometer } \\
& \left.\begin{array}{r}
\mathrm{I}=2,40 \\
\mathrm{II}=2,20
\end{array}\right\}=2,3 \% \mathrm{O}_{2}
\end{aligned}
$$


A. Differentialmanometer I bei $17^{\circ}$ im Wasserbade.

In die Birne ca. $0,1 \mathrm{ccm}$ Blut.

$\begin{array}{ccccc} & & \begin{array}{c}\text { In die linke Birne } \\ \text { das gesatttigte Blut }\end{array} & \begin{array}{c}\text { In die rechte Birne das } \\ \text { zu untersuch. Blut }\end{array} & \text { Dlfferenz } \\ \text { Gleichgewicht bei . . . . . } & \mathbf{7 , 6 5} & \mathbf{7 , 6 0} & \\ \text { Nach dem Schütteln . . . . } & 6,63 & \mathbf{8 , 6 0} & 2,02 \\ \text { Kaliumferricyanid rechts, } & & & \\ \text { Hähne vorher geöffnet . . } & 7,80 & 7,46 & 0,29\end{array}$

B. Differentialmanometer II bei $17^{\circ}$ im Wasserbade.

In die Birne ca. $0,1 \mathrm{ccm}$ Blut.

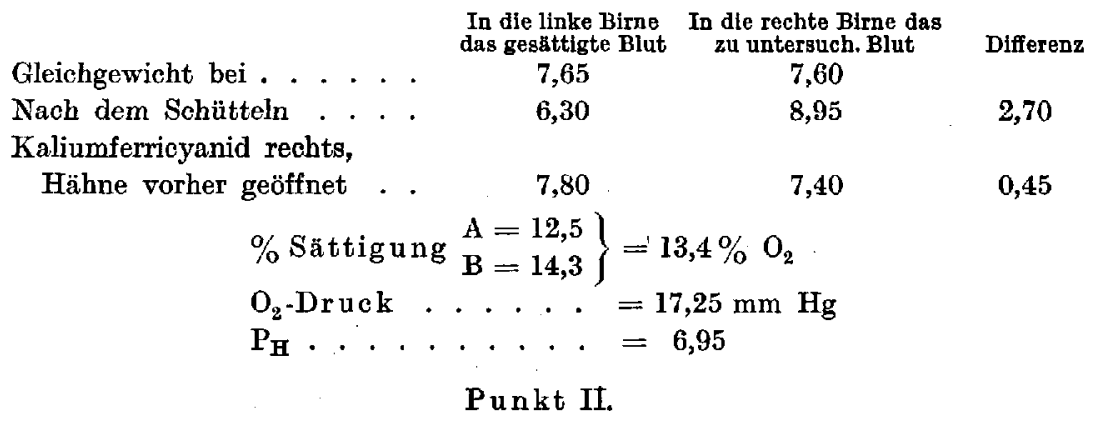

2,0 ccm Herzblut im Tonometer, daselbst ununterbrochen $25 \mathrm{Min}$. geschüttelt.

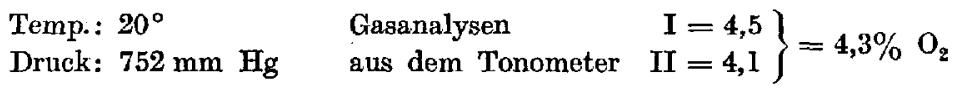

Differentialmanometer bei $17^{\circ} \mathrm{im}$ Wasserbade.

In die Birne ca. $0,1 \mathrm{cem}$ Blut.

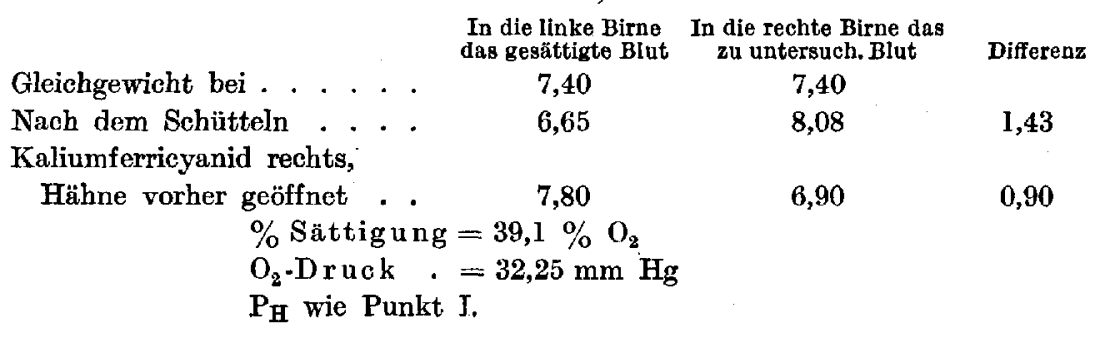

Fall Nr. 54.

Brasem, Margarete, Zwillingskind, Frühgeburt im ca. 7.-8. Schwangerschaftsmonat. Geb.-Gewicht 1150 g. Am 2. Lebenstage, 5. X. 14 ins K.-A.-V.-H. gebracht. Starke Untertemperatur $35,4^{\circ}$. Trotz Wärmewanne starke Neigung zur Untertemperatur. Bekam kleine Mengen Frauenmilch, bis $300 \mathrm{~g}$ täglich, per Sonde. Am 9. Lebenstage wurden die Stühle dünn, allmählich immer häufiger, am 16. ganz spritzend, mit vielem Schleim untermischt. Langsame Gewichtsabnahme. Ganz benommen, Gewicht $960 \mathrm{~g}$ bei Exit us, der nach langdauernder Agonie am 22. $X$. eintrat.

22. X. $4^{\text {h }}$ nachm. Sinuspunktion unmittelbar vor dem Exitus. 
Blut, aktuelle Reaktion . . . . . . . . . 6, 6,45

" $\mathrm{CO}_{2}$-freies . . . . . . . . . . . . 6.58

Gleich nach dem Exitus Selztion.

\section{Acidität der Organe.}

Gehirnsubstanz, ungekocht . . . . . . . . . 6,01

gekocht . . . ........ . 6,07

Muskel, Oberschenkel, ungekocht . . . . . . . 5,95

gekocht . . . . . . 6,4

Leber, ungekocht . . . . . . . . . . . 6,12

gekocht . . . . . . . . . 6, 6,

Milz, ungekocht . . . . . . . . . . . 6, 600

gekocht . . . . . . . . . . 6, 6, 6

Niere, ungekocht . . . . . . . . . . 5,67

gekocht ........... 5,83

Acidität des Magen- und Darminhaltes.

Magen, leer, Schleimhaut mit destill. Wasser gewaschen,

Waschwasser . . . . . . . . . . . . 5,53

Duodenum, Abschnitt von 30-60 cm unterhalb Pylorus gelblichbraune Brühe . . . . . . . . . . 4,26

Ileum, Abschnitt vor dem Coecum, Inhalt wie vor . $\quad 5,65$

Colon transversum, gelblichbraune Schleimflocken . 5,95

Rectum, gelblichbraun, zähe Schleimpartikel . . 5.95

Sektion ergab: starke hämorrhagische Injektion der Magen- und Daımschleimhaut.

Die vorstehenden Zahlen sind auf Kurve 11, I S. 310 graphisch dargestellt.

Fall Nr. 55.

Maudt, Else, Frühgeburt, am 2. XII. 1914 im Alter von 24 Tagen mit einem Gewicht von $1900 \mathrm{~g}$ an kongenitalem Herzfehler gestorben. Über das weitere Klinische und über die Versuche mit Säuremilch siehe $\mathbf{S}$. $\mathbf{3 8 0}$.

2. XII. $1^{\text {h }}$ mittags Exitus.

Sektion und Herzpunktion gleich danach.

Blut, aktuelle Reaktion .......... . 7,01

Muskel, ungekocht . . . . . . . . . . . . . . 6,04

gekocht . . . . . . . . . . . 6,28

Gehirn, ungekocht . . . . . . . . . . . 6,71

gekocht . . . . . . . . . . . 6,71

Leber, ungekocht. . . . . . . . . . . . 6,42

gekocht . . . . . . . . . 6, 6,59

Milz, ungekocht ............ . 6, 6, . . . . . .

gekooht . . . . . . . . . 6,37

Niere, ungekocht.......... . 6,52

gekocht . . . . . . . . . . 6,51

Magen- und Darminhalt:

Magen, milchige Flüssigkeit . . . . . . . . 5,88 
Duodenum, Abschnitt $60-80 \mathrm{~cm}$ unterhalb Pylorus, gelber Brei . . . . . . . . . . . . . . 5,85

Coecum, gelber Brei . . . . . . . . . . . . 5,93

Rectum, gelblichgrüner Brei . . . . . . . . . 5,58

Aus diesen Zahlen wurden mittels der Gleichgewichtsformel

$$
\begin{aligned}
n & =2,4 \\
K & =0,00016
\end{aligned}
$$

gefunden und mit Hilfe dieser Werte zwei weitere Punkte auf der Kurve berechnet, und zwar:

$$
\begin{array}{ll}
\text { Punkt III: } & \% \text { Sättigung }=75,0 \% \mathrm{O}_{2} \text { bei } \\
& \mathrm{O}_{2} \text {-Druck }=60,3 \mathrm{~mm} \mathrm{Hg} \\
\text { Punkt IV: } & \% \text { Sättigung }=85,0 \% \mathrm{O}_{2} \text { bei } \\
& \mathrm{O}_{2} \text {-Druck }=78,3 \mathrm{~mm} \mathrm{Hg}
\end{array}
$$

Bemerkung: Aus diesen Zahlen gebaute Dissoziationskurve hat einen auffallend niedrigen Verlauf, wie auch bei anderen Frühgeburten.

\section{Fall Nr. 56.}

Bail, Helmut, am 24. IX. 14 mit ca. $2650 \mathrm{~g}$ einen Monat zu früh geboren. Wegen mangelhaften Gedeihens im Alter von I Mon. am 20. X. zur Aufnahme gebracht. Status: deutlich abgemagertes, schlaffes Kind, dem Aussehen nach eine Frühgeburt, Gewricht 2115 g. Haut trocken, bläschenförmig abschilfernd an den Plantae und Palmae. Schnieft stark. Wassermann positiv. Trotz Frauen. milch ständige Abnahme; Stühle etwas zerfahren. Vom 28. $\mathrm{X}$. anfangend rapider Verfall und am 30. X. nach langandauernder Agonie Exitus.

30. X. Herzpunktion und Sektion gleich danach.

Blut, aktuelle Reaktion . . . . . . . . . . 6,29

$\mathrm{CO}_{\mathrm{z}}$-freies . . . . . . . . . . 6,60

Die Sauerstoff-Dissoziationskurve ergibt sich aus dem nachfolgenden

\begin{tabular}{|c|c|c|c|}
\hline Heichgewicht bei & $\begin{array}{c}\text { In die linke Birne } \\
\text { die gesăttigte Blutlösg. } \\
7,65\end{array}$ & $\begin{array}{c}\text { In die rechte Birne die } \\
\text { zu untersuch. Blutlösg. } \\
\quad 7,60\end{array}$ & Differenz \\
\hline Tach dem Schütteln & 6,80 & 8,36 & 1,61 \\
\hline liumferricyanid rechts, & & & \\
\hline Hähne vorher nicht geöffnet & 7,82 & 7,42 & 0,35 \\
\hline \multicolumn{4}{|c|}{ Differentialmanometer II im Wasserbade bei $17^{\circ}$. } \\
\hline & $\begin{array}{c}\text { In die lnke Birne } \\
\text { die gesattigte Blutlösg. } \\
\mathbf{7 , 4 4}\end{array}$ & $\begin{array}{l}\text { In die rechte Birne die } \\
\text { zu untersuch. Blutiôsg. } \\
\quad 7,42\end{array}$ & Differenz \\
\hline ch dem Scl & 6,82 & 8,38 & 1,58 \\
\hline
\end{tabular}

Punkt 1.

$3,0 \mathrm{ccm}$ Herzblut im Tonometer, daselbst ununterbrochen $25 \mathrm{Min}$. bei $38^{\circ}$ geschüttelt.

$$
\left.\begin{array}{lll}
\text { Temp.: } 18^{\circ} & \text { Gasanalysen } & \mathrm{I}=2,6 \\
\text { Druck: } 750 \mathrm{~mm} \mathrm{Hg} & \text { aus dem Tonometer } & \text { II }=2,5
\end{array}\right\}=2,6 \% \mathrm{O}_{2}
$$

Differentialmanometer I im Wasserbade bei $17^{\circ}$. 
Kaliumferricyanid rechts,

Hähne vorher nicht geöffnet

$$
\begin{aligned}
& \left.\% \text { Sättigung } \begin{array}{rl}
\mathrm{I} & =18,0 \\
\mathrm{II} & =18,1
\end{array}\right\}=18,1 \% \mathrm{O}_{2} \\
& \mathrm{O}_{2} \text {-Druck . . . }=19,5 \mathrm{~mm} \mathrm{Hg} \\
& \mathrm{P}_{\mathbf{H}} \text {........ }=6,51
\end{aligned}
$$

\begin{tabular}{|c|c|c|c|}
\hline $\begin{aligned} \text { Temp.: } & 18^{\circ} \\
\text { Druck: } & 750 \mathrm{~mm} \mathrm{Hg} \\
& \text { Differentialman }\end{aligned}$ & $\begin{array}{l}\text { Gasanalysen } \\
\text { aus dem Tonomete } \\
\text { ometer I bei } 17^{\circ} \mathrm{im}\end{array}$ & $\left.\begin{array}{r}I=4,4 \\
I I=4, I\end{array}\right\}=4,3^{\circ}$ & \\
\hline Gleichgewicht bei & $\begin{array}{l}\text { In die linke Birne } \\
\text { die gesăttigte Blutlögg. } \\
7,65\end{array}$ & $\begin{array}{l}\text { In die rechte Birne die } \\
\text { zu untersuch. Blutlösg. } \\
7,60\end{array}$ & Differenz \\
\hline Nach dem Schütteln & 6,97 & 8,21 & 1,39 \\
\hline $\begin{array}{l}\text { aliumferrioyanid rechts, } \\
\text { Hähne vorher nicht geöffnet }\end{array}$ & 7,91 & 7,28 & 0,58 \\
\hline
\end{tabular}

$3,0 \mathrm{ccm}$ Herzblut im Tonometer, daselbst ununterbrochen $25 \mathrm{Min}$. bei $38^{\circ}$ ge-

\begin{tabular}{|c|c|c|c|}
\hline & $\begin{array}{l}\text { In die linke Btrne } \\
\text { die gesăttigte Blutlősg. }\end{array}$ & $\begin{array}{l}\text { In die rechte Birne die } \\
\text { zu untersuch. Blutlösg. }\end{array}$ & Differenz \\
\hline Gleichgewicht bei . . . . . . & 7,40 & 7,40 & \\
\hline Nach dem Schütteln & 6,70 & 7,98 & 1,28 \\
\hline Kaliumferricyanid rechts, & & & \\
\hline Hähne vorher nicht geöffnet & 7,70 & 6,94 & 0,76 \\
\hline \multicolumn{4}{|c|}{$\%$ sättigung $\left.\begin{array}{rl}\mathrm{I} & =29,4 \\
\mathrm{II} & =37,0\end{array}\right\}=33,2 \% \quad \mathrm{O}_{2}$} \\
\hline $\begin{array}{l}\mathrm{O}_{2} \text {-Druck } \\
\mathrm{P}_{\mathbf{H}} \ldots . .\end{array}$ & $\begin{array}{l}\cdots \cdots \\
\cdots \cdots\end{array}$ & $\begin{array}{l}3 \mathrm{~mm} \mathrm{Hg} \\
51\end{array}$ & \\
\hline
\end{tabular}
schüttelt.

Differentialmanometer II im Wasserbade bei $17^{\circ}$

$3,0 \mathrm{ccm}$ Herzblut im Tonometer, Schütteln wie bei Punkt II.

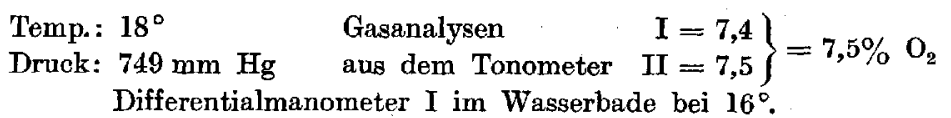

Gleichgewicht bei . . . . . In die linke Birne die In die rechte Birne zu untersuch. Blutlösg. die gesättigte Blutlösg. Differenz $\begin{array}{llll}\text { Nach dem Schütteln . . . } & 8,00 & 7,15 & 0,8 \mathrm{I}\end{array}$ Kaliumferricyanid links,

Hähne vorher nicht geöffnet $7,08 \quad 8,08$

Differentialmanometer II im Wasserbade bei $16^{\circ}$.

Gleichgewicht bei :

Nach dem Schütteln:

Kaliumferricyanid reohts,

Hähne vorher nicht geöffnet $\begin{array}{cc}\text { In die linke Birne } & \text { In die rechte Birne das } \\ \text { das gesättigte Blnt } & \text { zu untersuch. Blut }\end{array}$

$$
7,35
$$

6,92

7,35

7,68
6,75

1,13 
und Intoxikationsacidosis in ihren Beziehnngen zueinander.

$$
\begin{aligned}
& \left.\% \text { sättigung } \begin{array}{rl}
\mathrm{I} & =56,2 \\
\mathrm{II} & =60,0
\end{array}\right\}=58,1 \% \mathrm{O}_{2} \\
& \mathrm{O}_{2} \text {-Druck ..... }=56,3 \mathrm{~mm} \mathrm{Hg} \\
& \mathrm{P}_{\mathrm{H}} \ldots \ldots \ldots . \ldots=6,53
\end{aligned}
$$

Mit Hilfe der obigen Zahlen wurden in der Gleichgewichtsformel $\frac{y}{100}=\frac{K \times x^{n}}{1+K \times x^{n}}$ die Werte für $n$ und $K$ berechnet:

$$
\begin{aligned}
\boldsymbol{K} & =0,000125 \\
\boldsymbol{n} & =\mathbf{1 , 8 2}
\end{aligned}
$$

und danach durch dieselbe Formel zwei weitere Punkte auf der Dissoziationskurve rechnerisch bestimmt. Und zwar wurden gefunden:

$$
\begin{array}{ll}
\text { 4. Punkt: } & \% \text { Sättigung }=70,0 \% \mathrm{O}_{2} \text { bei } \\
& \mathrm{O}_{2} \text {-Druck }=23,5 \mathrm{~mm} \mathrm{Hg} \\
\text { 5. Punkt: } & \% \text { Sättigung }=80,0 \% \mathrm{O}_{2} \text { bei } \\
& \mathrm{O}_{2} \text {-Druck }=31,7 \mathrm{~mm} \mathrm{Hg}
\end{array}
$$

Alle diese 5 Punkte sind in Fig. 8 in einer Dissoziationskurve graphisch dargestellt.

Acidität der Organe.

Gehirnflüssigkeit, trübe mit Gehirnpartikeln vermischt $\quad 6,31$

Leber, ungekocht . . . . . . . . . . 6,27

gekacht . . . . . . . . . 6,32

Milz, ungekocht . . . . . . . . . . 6,62

gekocht ............ . 6, 6, . . . .

Aciditat des Magen- und Darmkanals.

Magen, ca. $60 \mathrm{ccm}$ milchige Flüssigkeit . . . . . 6,27

Duodenum, Abschnitt 70-100 cm unterhalb Pylorus,

gelbliche, schleimige, flockige Flüssigkeit . . . . 5,64

Ileum, oberer Abschnitt, gelbe breiige Massen . . . 6,74

Ileum, kurz vor Coecum, Inhalt wie vor . . . . . 6,48

Rectum, gelbe breig-salbige Massen . . . . . . . 6,55

Die Sektion ergab Hypertrophia lienis, Osteochondritis Iuetica.

Die Werte für die Organacidität sind auf Fig. 11, III S. 310 graphisch dargestellt.

Fall Nr. 57.

Modersitzky, Marie, 16. IX. 14 mit $1500 \mathrm{~g}$ ca. 2 Mon. zu früh geboren. Kommt im Alter von 10 Std. zur Aufnahme. Status: stark abgekühlte Frühgeburt. Temp. $28^{\circ}$. Ödeme, blau, bekommt in der Wärmewanne allmählich Temperatur bis $38^{\circ}$, die Atmung will aber nicht in Gang kommen, und das Kind stirbt 12 Std. nach der Aufnahme im Alter von 22 Std.

17. IX. Herzpunktion und Sektion gleich nach dem Exitus.

Blut, aktuelle Reaktion . . . . . . . . 6,65

Gehirnpunktat, trübe Flüssigkeit mit Gehirnmassen vermischt . . . . . . . . . . . 6,65

Muskulatur, Oberschenkel, frisch . . . . . . 6,41 


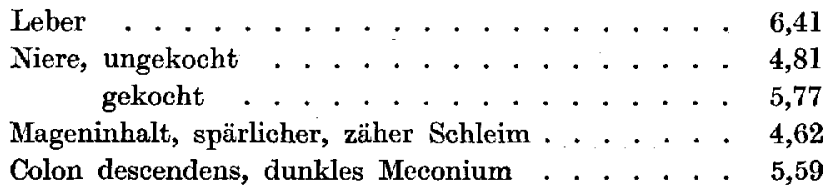

Sektionsdiagnose: Frühgeburt, Atelektase (?) der beiderseitigen Lungenunterlappen.

Außerdem wurden noch zwei Punkte anf der $\mathrm{O}_{2}$-Dissoziationskurve des Blutes bestimmt.

\section{Punkt I.}

3,0 com Herzblut im Tonometer, daselbst ununterbrochen $25 \mathrm{Min}$. geschüttelt.

Temp.: $19^{\circ}$

Gasgemisch ... . $=5,2 \% \mathrm{O}_{2}$

Druck: $750 \mathrm{~mm} \mathrm{Hg}$

Gasanalyse $\quad \mathrm{I}=5,0 \% \mathrm{O}_{2}$

aus dem Tonometer I

A. Differentialmanometer I bei $17^{\circ}$ im Wasserbade.

In die Birne ca. $0,1 \mathrm{ccm}$ Blut.

In die linke Birne In die rechte Birne das das gesättigte Blut zu untersuch. Blut Differenz

Gleichgewicht bei . . . . . $\mathbf{7 , 6 5}$ 7,60

Nach dem Schütteln . 7,39

7,90

Kaliumferricyanid rechts,

Hähne vorher nicht geöffnet

9,20

6,10

$$
\begin{aligned}
& \% \text { Sättigung }=86,6 \% \mathrm{O}_{2} \\
& \mathrm{O}_{2} \text {-Druck }=37,5 \mathrm{~mm} \mathrm{Hg} \\
& \mathrm{P}_{\mathrm{H}} . . . .=7,53
\end{aligned}
$$

Punkt II.

Gasanalysen aus dem Tonometer $2,7 \% \mathrm{O}_{2}$.

\begin{tabular}{|c|c|c|c|}
\hline & $\begin{array}{l}\text { In die linke Birne } \\
\text { das gestitigte Blut }\end{array}$ & $\begin{array}{l}\text { In die rechte Birne das } \\
\text { zu untersuch. Blut }\end{array}$ & Differenz \\
\hline Gleichgewicht bei . . . . . . & 7,40 & 7,40 & \\
\hline Nach dem Schütteln & 6,32 & 8,40 & 2,08 \\
\hline \multicolumn{4}{|l|}{ Kaliumferricyanid rechts, } \\
\hline Hähne vorher nicht geöffnet & 8,40 & 6,30 & 2,10 \\
\hline $\begin{array}{l}\% \mathrm{Sä} \\
\mathrm{O}_{2} \cdot \mathrm{Dr} \\
\mathbf{P}_{\mathrm{H}} \mathrm{w}\end{array}$ & $\begin{array}{l}\text { ig ung }=50,0 \% \\
\mathrm{ck}=20,25 \\
\text { Punkt I. }\end{array}$ & $\begin{array}{l}\mathrm{O}_{2} \\
\mathrm{~m} \mathrm{Hg}\end{array}$ & \\
\hline
\end{tabular}

B. Differentialmanometer II bei $17^{\circ}$ im Wasserbade.

In die Birne ca. 0,1 ccm Blut.

Fall Nr. 58.

Frank, Käte, am 16. XII. 14 im 7. Schwangerschaftsmonat mit $1100 \mathrm{~g}$ geboren, kam im Alter von $24 \mathrm{Std}$. zur Aufnahme. Stark abgekühlt, Ödeme. Hatte bisher nur etwas Fencheltee bekommen, Versuche mit Sonde $5 \mathrm{~g}$ Frauenmileh zu geben mißlangen, die Milch mit blutigem Schleim vermischt wird gleich wieder herausgebrocben. Atmung scblecht, stirbt nach $16 \mathrm{Std}$. im Alter von $11 / 2$ Tagen. 
und Intoxikationsacidosis in ihren Beziehungen zueinander.

18. XII. Herzpunktion und Sektion gleich nach dem Fxitus.

Blut, aktuelle Reaktion . . . . . . . . . . 6,52

, $\mathrm{CO}_{2}$-freies . . . . . . . . . . . 7,35

Muskel, ungekocht. . . . . . . . . . . 6,67

gekocht.. . . . . . . . . . . . 7,19

Leber, ungekocht . . . . . . . . . 7,15

gekocht . . . . . . . . . 7,17

Milz, ungekocht.............. $7, \mathbf{7 3 3}$

gekocht . . . . . . . . . . . . 7,33

Niere, ungekocht . . . . . . . . . . 7,19

gekocht . . . . . . . . . . . . . . 7,59

Mageninhalt: 1-2 cem gelblich-schleimige Flüssigkeit 6,39

Dünndarm, zur Hälfte Meconium, zur Hälfte schlei-

miges Milchgerinsel. . . . . . . . . . . . 6,10

Dickdarm, dunkelgrünes Meconium . . . . . . 5,70

Sektion ergab kleine atelektatische Partien und multiple Embolien.

Bemerkung: Mit Ausnahme der Werte von $\mathrm{P}_{\mathrm{H}}$ für das Blut sind die Werte in diesem Falle unerwartet hoch, im Gegensatz zu allen anderen Frühgeburten.

Fall Nr. 59.

Luste, Paul, Zwillingskind, im 7. Schwangerschaftsmonat mit $1650 \mathrm{~g}$ ge. boren, kam 2 Std. alt zur Aufnahme. Status: Frühgeburt mit bläulich-rosa verfärbter Haut, Temperatur $37^{\circ}$. In die Wärmewanne, atmet schlecht und stirbt in einem asphyktischen Anfall im Alter von 12 Std.

30. VIII. 14 Herz- und Gehirnpunktion gleich nach dem Exitus.

Blut, aktuelle Reaktion . . . . . . . . . . 6,34

" $\mathrm{CO}_{2}$-freies . . . . . . . . . . . 6,80

Gehirnpunktat, hauptsächlich Gehirnmassen . . . 6,33

Die Dissoziationskurve des $\mathrm{O}_{2}$-Hämoglobins ergibt sich aus den folgenden Bestimmungen, die gleich im Herzblut vorgenommen wurden.

\section{Punkt.}

2,0 ccm Herzblut im Tonometer, daselbst ununterbrochen $25 \mathrm{Min}$. bei $38^{\circ}$ geschüttelt.

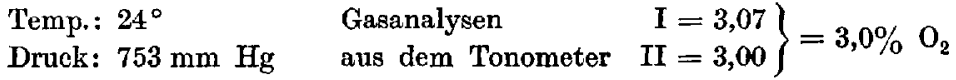

In die Birne ca. 0,1 ccm Blut.

Differentialmanometer bei $21^{\circ}$ im Wasserbade.

\begin{tabular}{|c|c|c|c|}
\hline & $\begin{array}{l}\text { In die linke Birme } \\
\text { das gesattigte Blut }\end{array}$ & $\begin{array}{l}\text { In die rechte Birne das } \\
\text { zu untersuch. Blut }\end{array}$ & Dlfferenz \\
\hline Gleichgewicht bei . . & 7,70 & 7,65 & \\
\hline Nach dem Schütteln & 6,65 & 8,68 & 2,08 \\
\hline \multicolumn{4}{|l|}{ Kaliumferricyanid rechts, } \\
\hline Hähne vorher nicht geöffnet & 8,64 & 6,73 & 1,86 \\
\hline $\begin{array}{l}\% \mathrm{Sä} \\
\mathrm{O}_{2}-\mathrm{D} \\
\mathrm{P}_{\mathrm{H}} .\end{array}$ & $\begin{aligned} \operatorname{igung} & =47,2 \% \\
& =22,5 \mathrm{~m} \\
\mathrm{ck} & =6,80\end{aligned}$ & $\begin{array}{l}\mathrm{O}_{2} \\
\mathrm{~m} \mathrm{Hg}\end{array}$ & \\
\hline
\end{tabular}


II. Punkt.

2,0 ccm Herzblut im Tonometer, geschüttelt wie bei Punkt I.

$\left.\begin{array}{lll}\text { Temp.: } 24^{\circ} & \text { Gasanalysen } & \mathrm{I}=5,45 \\ \text { Druck: } 753 \mathrm{~mm} \mathrm{Hg} & \text { aus dem Tonometer } & \mathrm{II}=5,30\end{array}\right\}=5,4 \% \mathrm{O}_{2}$ Differentialmanometer II bei $21^{\circ}$ im Wasserbade.

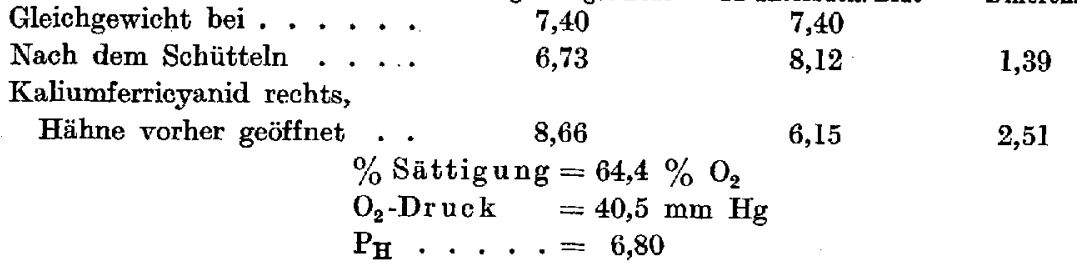

Aus der Formel $\frac{Y}{100}=\frac{K \cdot x^{n}}{1+K \cdot x^{n}}$ wurden die Werte für $n$ und $K$ berechnet:

$$
\begin{aligned}
n & =1,2 \\
K & =0,0208 .
\end{aligned}
$$

Die aus den obigen Zahlen aufgebante $\mathrm{O}_{2}$-Dissoziationskurvo des Blutes ist auf Fig. 24, II S. 330 dargestellt.

\section{Hunger- u. a. Versuehe.}

Einfluß des Hungers anf die Acidität des Urins und des Stuhls und auf die Regulationsbreite der $\mathrm{CO}_{2}$ im Blute.

Fall Nr. 60.

Jacoby, Hubert, gesundes Ammenkind, geb. I6. IX. 14. Gewicht $2700 \mathrm{~g}$. Zartes, etwas mageres Kind, physiologische Abnahme $150 \mathrm{~g}$. Regelmäßige Zunahme vom 3. Lebenstage an. Bekommt in den ersten Tagen nur kleinere Mengen von $90-400 \mathrm{~g}$ Frauenmilch, später $700-800 \mathrm{~g}$ bis zum 18. XI., von da an bis zum 1. XII. neben Frauenmileh $1 / 2$ Milch, vom 2. XII. (vom ca. $21 / 2$. Lebensmonat an) anfangend nur künstliche Ernährung, zuerst $1 / 2$ Milch, später $2 / 3$ Milch mit Beikost. Wird 10 Mon. alt, am 21. VII. 1915 mit $6760 \mathrm{~g}$ entlassen.

Bei dem Kinde wurde die Dissoziationskurve des Nabelschnurblutes, des Blutes im Alter von 5 Tagen und 2 Monaten bestimmt. Siehe Näheres S. 384.

Außerdem wurden bei dem Kinde Hungerversuche vorgenommen, wie aus dem Folgenden hervorgeht:

I. Hungerversuch (mit $1 / 2$ Ringerlösung $+1 / 2$ Tee):

Dauer des Hungers vom 11. I. $156^{\mathrm{h}}$ vorm. bis 13 . I. $15 \mathrm{1}^{\mathrm{h}}$ mittags $=2$ Tage 7 Stunden.

Während dieser Zeit trank das Kind von einer Lösung, die zur Hälfte aus Ringerlösung und zur Hälfte aus Tee bestand; am 11. I. im ganzen $720 \mathrm{~g}$. Am 12. I. $980 \mathrm{~g}$ derselben Mischung, am 13. I. vorm. $80 \mathrm{~g}$. Die Nahrung bestand vor und nach dem Versuch aus $1 / 2$ Milch mit $4 \%$ Rohrzucker und $2 \%$ Malzextrakt. Das Gewicht des Kindes stieg bei Beginn des Versuches (11. I.) von $4740 \mathrm{~g}$ bis zum Ende des Versuches (13. I.) auf $4900 \mathrm{~g}$. Nachdem das Kind wieder Nahrung bekam, 
fing es an abzunehmen, niedrigstes Gewicht am 15. I. $=4640 \mathrm{~g}$. Am I. Tage des Versuches schrie das Kind viel, am 2. und 3. Tage war es schon ruhiger und trank die Ringer + Tee-Lösung sehr gut. Die Temperatur bewegte sich in der ganzen Zeit zwischen 36,8-37 . Die Veränderungen in der Acidität des Stuhls, Urins und des Blutes gehen aus folgenden Zahlen hervor:

Vor dem Hunger:

I. StuhI und Urin.

10. I. $8^{\mathrm{h}}$ vorm. Urin. . . . . . . . . . . 6,69

Stuhl, weißliche Salbe . . . 7,86

$2^{\text {n }}$ nachm. Stuhl, wie vor . . . . . . . 8,32

Urin ... . . . . . 6,68

11. I. $8^{\mathrm{h}}$ vorm. Urin ........... 6, 6,

Stuhl, weißliche Paste . . . 7,97

Während des Hungers:

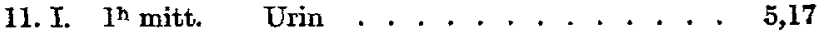

$5^{\mathrm{h}}$ nachm. Urin ...........5,15

12. I. $9^{\mathrm{h}}$ vorm. Urin . . . . . . . . . . . . 5,39

$\mathrm{I}^{\mathrm{h}}$ mitt. Urin . . . . . . . . 5,09

$\mathbf{5}^{\mathrm{h}}$ nachm. Urin . . . . . . . . 5, 5,

$5^{\text {h }}$ nachm. Stuhl, zum erstenmal, wässerig, in

die Windel gegangen . . . 0,00

13. I. $9^{\mathrm{h}}$ vorm. Urin . . . . . . . . . . 4 4,88

Von $1^{\text {h }}$ ab wieder Nahrung:

$3^{\mathrm{h}}$ nachm. Urin . . . . . . . . . . 5,37

$5^{\mathrm{h}}$ nachm. Urin . . . . . . . . 5,71

14. I. 9h vorm. Urin . . . . . . . . . . 6,97

$3^{\text {h }}$ nachm. Urin . . . . . . . . . . 6,89

$6^{\mathrm{h}}$ nachm. Urin ........... 7,44

15. I. $9^{n}$ vorm. Urin . . . . . . . . . . . . 7,39

$11^{\text {b }}$ vorm. Stuhl, weißliche harte Paste. . 7,51

17. I. 9b vorm. Stuhl, wie vor . . . . . . . 8,29

20. I. $9^{\mathrm{h}}$ vorm. Stuhl, wie vor . . . . . . . 7,51

23. I. $9^{\text {h }}$ vorm. Stuhl, wie vor . . . . . . 8,44

Urin ......... 6,30

II. Blut.

11. I. $8^{\text {h }}$ vorm. ( 2 Std. nach der letzten Flasohe)

aktuelle Reaktion . . . . 7,53

13. I. l $^{\mathrm{h}}$ mitt. (am Ende des Hungers)

aktuelle Reaktion .... 7,51

II. Hungerversuch (40stündiger absoluter Hunger):

Beginn des Hungers 19. II. $154^{\mathrm{h}}$ nachm. bis 21. ПI. $158^{\mathrm{h}}$ vorm. $=40 \mathrm{Stun}$ den. (Nahrung $900 \mathrm{~g}: 1 / 2$ Milch mit 5\% Rohrzucker vor und nach dem Hunger.)

Das Kind war anfangs etwas unruhig, gegen Ende des Hungers beruhigte es sich aber und schlief die letzte Nacht ganz ruhig. Temperatur am 19. II. $5^{\mathrm{h}}$ nachm. bei Beginn des Hungers $37,5^{\circ}$, am 20. II. $5^{\mathrm{h}}$ nachm., die höohste Tem- 
peratur während des Hungers, 37,8 ${ }^{\circ}$. Am 21. II. abends 37,3 $3^{\circ}$ Das Gewicht sank während des Versuches von $4900 \mathrm{~g}$ auf $4550 \mathrm{~g}$ herab. Die Veränderungen in der Acidität des Stuhls und des Urins gehen aus folgenden Zahlen hervor:

\section{StuhI und Urin.}

Vor dem Hunger:

19. II. $9^{\text {h }}$ vorm. Stuhl, weißlichbraun, bröcklig $\quad 7,72$

Urin ......... . 6,13

$5^{\mathrm{h}}$ nachm. Stuhl, wie vor ..... . $\mathbf{7 , 9 2}$

Urin .......... 6,03

Während des Hungers:

21. II. $6^{\mathrm{h}}$ vorm. Urin . . . . . . . . . . 5,45

Von $8^{\mathrm{h}}$ früh ab wieder Nahrung:

21. II. $5^{\text {h }}$ nachm. Urin . . . . . . . . . . . . . 5,81

Stuhl, braun, zäh knollig . . . $\quad 6,79$

22. II. $8^{\mathrm{h}}$ vorm. Urin . . . . . . . . . . 5,89

$5^{\text {h }}$ nachm. Stuhl, gelbliche, weißliche Salbe 7,02

Urin . . . . . . . . . 6,72

23. II. $5^{\text {h }}$ nachm. Stuhl, bräunliche, knollige Paste $\mathbf{7 , 7 6}$

24. II. $5^{\mathrm{h}}$ nachm. Stuhl, wie vor . . . . . . . 7,75

25. II. $5^{\mathrm{h}}$ nachm. Urin . . . . . . . . . . . . $\mathbf{7 , 1 4}$

26. II. $8^{\mathrm{h}}$ vorm. Urin . . . . . . . . . . . 7,29

27. II. $9^{\mathrm{h}}$ vorm. Urin . . . . . . . . . . 6,79

Stuhl, braune, brüchige Massen - 8,52

28. II. 9h vorm. Urin . . . . . . . . . . . . . 6,41

1. III. $5^{\text {h }}$ nachm. Urin . . . . . . . . . . . . . 6,06

2. III. $5^{\text {h }}$ nachm. Stuhl, bräunliohe, brüchige Massen 8,25

II. Blut.

19. II. $6^{\text {h }}$ nachm. (kurz nach Beginn des Hungers)

$\left.\begin{array}{lll}\text { aktuelle Reaktion . . . . . . . . . } & 7,57 \\ \mathrm{CO}_{2} \text {-freies . . . } & 8,27\end{array}\right\}=70 \%$

21. II. $8^{\mathrm{h}}$ vorm. (am Ende des Hungers)

$\left.\begin{array}{lll}\text { aktuelle Reaktion . . . . . . . . . } & 7,59 \\ \mathrm{CO}_{2} \text {-freies . . . } 899\end{array}\right\}=50 \%$

Die Veränderungen in der Acidität des Stuhles und des Urins in beiden Versuchen sind auf Fig. 14 S. 314 graphisch dargestellt. Die Veränderungen des Blutes während des II. Hungerversuches auf der Fig. 15, I S. 315 und auf Fig. 16 S. 316.

Fall Nr. 61.

Lux, Hansi, gesunde Frühgeburt, ziemlich ausschließlich 6 Mon, lang mit Brustmilch ernährt: Geb. 12. IX, 14 etwa im 7. Schwangerschaftsmonat. Im Alter von 10 Tagen aus einer Entbindungsanstalt zu uns gebracht,

23. IX. 14. Status: Kleine, zierliche Frühgeburt von $1400 \mathrm{~g}$ Gewicht, wird mit Temperatur yon $31^{\circ}$ eingeliefert. Kommt in die Wärmewanne; in den ersten 10 Tagen starke Neigung zur Untertemperatur, später Monothermie um ca, $37^{\circ}$. 
Das Kind entwickelt sich gut und wird im Alter von 11 Mon. mit einem Gewicht von $5800 \mathrm{~g}$ bei $2 / 3$ Milch mit 5\% Rohrzucker nebst Beikost gesund entlassen.

Bei dem Kinde wurden folgende Hungerversuche vorgenommen:

I. Hungerversuch (mit $1 / 2$ Ringerlösung + 1/2 Tee):

Dauer des Hungers 2 Tage 7 Stunden, und zwar vom 11 . I. $156^{\mathrm{h}}$ vorm. bis 13. I. $151^{\text {h }}$ mittags. Die Nahrung bestand vor und nach dem Hunger aus $600 \mathrm{~g}$ Frauenmilch täglich. Während des Versuches trank das Kind von einem Gemisch $1 / 2$ Ringerlösung $+1 / 2$ Tee am 11. I. $450 \mathrm{~g}$, am 12. I. $530 \mathrm{~g}$ und am 13. I. $100 \mathrm{~g}$. Das Gewicht nahm zu, während der Versuchszeit von $3000 \mathrm{~g}$ bis $3180 \mathrm{~g}$, sank dann aber in den nächsten 5 Tagen auf $2900 \mathrm{~g}$ herab. Die Temperatur betrug ca. $36,6^{\circ}$ die ganze Zeit hindurch. Am ersten Tage unruhig, am zweiten ruhiger; trank die Mischung gern.

Vor dem Hunger:

\section{Stuhl und Urin.}

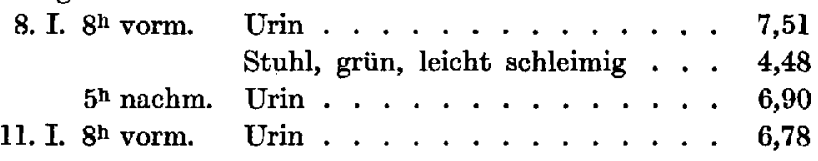

Während des Hungers:

$12^{\mathrm{h}}$ mitt. Urin . . . . . . . . . . 7,04

$4^{\text {h }}$ nachm. Stuhl, grün, schleimig .... 5,38

Urin, wasserklar . . . . . . 6,81

12. I. $8^{\text {h }}$ vorm. Urin ..... . . . . . . 5,04

$1^{\mathrm{h}}$ mitt. Urin . . . . . . . . . . 4,95

$5^{\text {h }}$ nachm. Urin . . . . . . . . . . 4,80

13. I. $8^{\text {h }}$ vorm. Urin . . . . . . . . . . 5,49

Von $1^{\text {th }}$ ab wieder Nahrung:

$3^{\text {h }}$ nachm. Urin . . . . . . . . . . . 5,36

$6^{\text {h }}$ nachm. Urin . . . . . . . . . 5, 5,

14. I. $8^{\mathrm{h}}$ vorm. Urin . . . . . . . . . 6,42

$3^{\text {h }}$ nachm. Urin . . . . . . . . . . . . 7,09

14. I. $3^{\text {h }}$ nachm. Stuhl (der erste seit 2 Tagen), bräun-

lich, etwas schleimig, dünn . . 4,50

$7^{\mathrm{h}}$ nachm. Urin . . . . . . . . . . . 6,86

15. I. $9^{\mathrm{h}}$ vorm: Urin . . . . . . . . . 8,56

Stuhl, gelb, salbig, schleimig . . $\quad 4,88$

17. I. $8^{\text {h }}$ vorm. Stuhl, grüngelb, schleimig . . . . 5,40

18. I. $8^{\text {h }}$ vorm. Stuhl, wie vor. . . . . . . . 5,09

Urin . . . . . . . . . 6,60

II, Blut.

11. I. (2 Std. nach der letzten Flasche)

aktuelle Reaktion . . . . . . . 7,48

13. I. $1^{h}$ mitt. (am Ende des Hungers)

aktuelle Reaktion . . . . . . 7,53 
II. Hungervers uch (40stündiger absoluter Hunger):

Beginn des Hungers 19. II. $154^{\mathrm{h}}$ nachm. bis 21 . II. $158^{\mathrm{h}}$ vorm. $=40 \mathrm{St}$ u n. den. Nahrung vor und nach dem Hunger $600 \mathrm{~g}$ Frauenmilch täglich.

Anfangs war das Kind sehr unruhig, beruhigte sich aber gegen Ende des Versuches; machte vor dem Ende des Hungers otwas müden Eindruek. Das Kind zeigte vorher eine auffallende Monothermie, morgens $36,8^{\circ}$ und abends $37,1^{\circ}$. Am 20. II. nach 24stündigem Hunger stieg die Temperatur allmählich bis $37,9^{\circ}$, hielt sich in dieser Höhe bis zum 21. II. $6^{\text {h }}$ vorm. und fiel danach im Verlaufe des 21. II. langsam bis zur Norm $37^{\circ}$ zurück. In den dem Hunger folgenden nächsten Tagen bestand die Monothermie wie vor dem Versuoh. Das Gewicht sank während des Hungers von $3600 \mathrm{~g}$ auf $3360 \mathrm{~g}$ herab. Die Veränderungen in der Acidität des Stuhles und des Urins gehen aus folgenden Zahlen hervor:

Vor dem Hunger:

I. Stuhl und Urin.

19. II. 9h vorm. Urin . . . . . . . . . . 7,01

Stuhl, dünn, grünlich-schleimig . $\quad 4,82$

Während des Hungers:

$4^{\mathrm{h}}$ nachm. Urin . . . . . . . 7,41

20. II. $9^{\text {h }}$ vorm. Urin . . . . . . . . . . . 5,51

$\mathbf{4}^{\mathrm{h}}$ nachm. Urin . . . . . . . . . . . . . 5,51

21. II. $7^{\text {h }}$ vorm. Urin .......... . 5,44

Von $8^{\text {h }}$ vorm, ab wieder Nahrung:

$=10^{\mathrm{h}}$ vorm. Urin . . . . . . . . . 5,44

$2^{\mathrm{h}}$ nachm. Urin . . . . . . . . 5,45

$4^{\text {h }}$ nachm. Stuhl, salbig, mit Schleim überzogen (2 Tage verstopft gewesen) $\quad 6,62$

22. II. $7^{\mathrm{h}}$ vorm. Urin ............. 5,42

$2^{\mathrm{h}}$ nachm. Urin . . . . . . . . 6,90

Stuhl, wie vor, nur mehr pastig . $\quad 6,72$

23. II. $5^{\text {h }}$ nachm. Stuhl, leicht schleimig, gelblichgrün 5,13

24. II. $12^{\mathrm{h}}$ mitt. Urin . . . . . . . . . . 8,06

Stuhl, wie vor . . . . . 5,04

26. II. $8^{\text {n }}$ vorm. Urin . . . . . . . . . . . . 7,90

$5^{\text {h }}$ nachm. Stuhl, wie vor . . . . . 4,98

27. II. $8^{\text {h }}$ vorm. Urin . . . ........ . 7,85

Stuhl, wie vor ...... . 4,94

28. II. $8^{\mathrm{h}}$ vorm. Urin . . . . . . . . . . . 7,61

1. III. $8^{\text {h }}$ vorm. Urin . . . . . . . . . . 7,69

Stuhl, grünlichgelbe Salbe . . 6,64

2. III. $5^{\mathrm{h}}$ nachm. Urin . . . . . . . . . . . 7,60

Stuhl, schleimig, etwas dünn . . 5,01

3. III. $5^{\text {h }}$ nachm. Stuhl, wie vor . . . . . . . 5,49

Urin : ...... 7,34

4. III. $8^{\text {h }}$ vorm. Stuhl, wie vor . . . . . . 4,91 


$$
\text { II. Blut. }
$$

19. II. $6^{\text {h }}$ nachm. (kurz nach Beginn des Hungers)

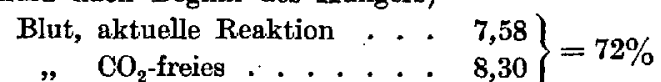

21. II. $8^{\text {h }}$ vorm. (am Ende des Hungers)

$$
\left.\begin{array}{cccc}
\text { Blut, } & \text { aktuelle Reaktion } . . . & 7,62 \\
, & \mathrm{CO}_{2} \text {-freies ...... } & 8,23
\end{array}\right\}=61 \%
$$

Die Veränderungen in der Acidität des Stuhles und Urins in beiden Versuchen sind auf Fig. 13, I und II S. 313, die Veränderungen des Blutes während des II. Versuches auf Fig. 15, II S. 315 und Fig. 16 S. 316 graphisch dargestellt.

\section{Fall Nr. 62.}

Koehler, Erika, Frühgeburt von $960 \mathrm{~g}$ Geburtsgewicht. Frauenmilchernährung 6 Mon. lang. Geb. 12. IX. 14, im 7. Schwangerschaftsmonat, Ursache zur Frihgeburt unbekannt. Wird aus einer Entbindungsanstalt im Alter von 7 Tagen zu uns überwiesen. Kleine Frühgeburt, Gewicht ca. $1000 \mathrm{~g}$, Länge $37,5 \mathrm{~cm}$. Das Kind kommt in die Wärmewanne, nimmt regelmäßig gut zu. Im Alter von $2 \mathrm{Mon}$. Gewicht $1600 \mathrm{~g}$, wird dann aber allmählich sehr blaß, der Hinterkopf wird sehr weich, das Gewicht nimmt trotzdem ununterbrochen zu, das Kind bleibt aber sehr schlaff. Kann im Alter von 51/2 Mon. nur kürzere Zeit den Kopf halten, mit dem Fortschreiten der Anämie starke Appetitlosigkeit. Im Alter von 6 Mon. Pyelitis und Bronchitis, aus welcher sich dann später eine Pneumonie entwickelt, an der das Kind nach langem Kranksein im Alter von 8 Mon. mit einem Gewicht von $3000 \mathrm{~g}$ am 7. V. 15 starb.

Bei dem Kinde wurden Untersuchungen bezüglich der Acidität in den verschiedensten Richtungen vorgenommen.

Aciditat des Urins und des Stuhles in den ersten Lebensmonaten.

\section{Versuch I.}

a. Der Urin von 24 Stunden wurde in einem mit etwas Toluol versetzten GefäB, das auf Eis gehalten wurde, gesammelt. Die Bestimmung der Acidität wurde $8^{\mathrm{h}}$ vorm. vorgenommen.
19. IX. . . . . . 5,97
26. IX. . . . . 5,18
5. X. . . . . . 5,55
12. $X . \cdots, \ldots . .58$
19. X. . . . . . 5,87
26. X. . . . . 5,62
2. XI. . . . . . 5,40
9. XI. . . . . . 5,43
16. XI. . . . 5,54
23. XI. . . . . 5,75
30. XI. . . . . . 5,94

1. XII. . . . . . . 6,11

3. XII. . . . . . . 5,98

7. XII. . . . 6 6,38

14. XII. . . . . . . 6,11

1. I. . . . . . . 7,02

2.1. . . . . 7,06

3. I. . . . . . 6,98

15. I. . . . . . 7,12

16. I. . . . . . . . . . 7,15

Spätere Werte siehe im Versuch II. 
b. Der Stuhl wurde jedesmal frisch untersucht. Er war'immer leicht schleimig, gelblich-grünlich.
19. IX. . . . . . 4,65
5. X. . . . . 4,90
12. X. . . . 4,95
26. X. . . . . 4,81
2. XI. . . . . 4,62
23. XI. . . . 4,60

7. XII. . . . . 4,58

14. XII. . . . . 4,76

5.1....... . 4,80

8. I. . . . . . 4,82

10. I. . . . . . 4,78

Diese $\mathrm{P}_{\mathbf{B}}$-Werte, sowohl die für den Urin als die für den Stuhl, sind auf Fig. 1 S. 293 graphisch dargestellt.

\section{Versuch II.}

1. Hungerversuch von $2^{1} / 2$ Tagen Dauer (mit $1 / 2$ Ringerlösung $+1 / 2$ Tee) vom 18. I. $1511^{\text {h }}$ abends bis zum 21. I. $9^{\text {h }}$ vorm. Das Kind hat am 19. und 20. I. je $420 \mathrm{~g}$, am 21. I. bis zum. Ende des Versuches nur $160 \mathrm{~g}$ obiger Mischung zum Trinken bekommen. Vor und nach dem Hunger täglich $420 \mathrm{~g}$ Frauenmilch. Das Gewicht blieb während des Versuches unverändert $2300 \mathrm{~g}$. Alter des Kindes bei Beginn des Versuches 4 Mon. Sehr blaß, Temperatur vor und während des Versuches unverändert 36,8 bis $37^{\circ}$. Trank mit Wohlbehagen die Mischung. Am Ende des Versuches sehr matt.

\section{Acidität des Stuhls und des Urins.}

Vor dem Hunger:

15. I. 9h vorm. Urin . . . . . . . . . 7,12

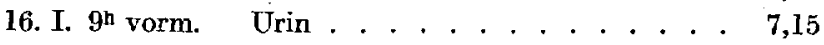

18. I. $9^{\mathrm{h}}$ vorm. Urin . . . . . . . . 6,96

$3^{\text {h }}$ nachm. Stuhl, grünlich, schleimig $\ldots . .5,11$

Während des Hungers:

19. I. $6^{\mathrm{h}}$ vorm. Urin ............ 6,69

$8^{\text {h }}$ vorm. Stuhl, wie vor. . . . . . . . 5,37

$5^{\mathrm{h}}$ nachm. Urin . . . . . . . . . . 6,60

Stuhl, wie vor. . . . . . . . 5,18

20. I. $9^{\mathrm{h}}$ vorm. Urin . . . . . . . . 6,11

$5^{\text {h }}$ nachm. Urin . . . . . . .... . 5,66

21. I. $8^{\text {h }}$ vorm. Stuhl, grünbraun, zäh, spärlich $\ldots 7,53$

$9^{\text {h }}$ vorm. wieder die Nahrung:

$9^{\mathrm{h}}$ vorm. Urin . . . . . . . . . . 5,80

$5^{\mathrm{h}}$ nachm. Urin . . . . . . . . . . . 5,77

Stuhl, grün und schleimig . . . 4,90

22. I. $9^{\text {h }}$ vorm. Urin . . . . . . . . . . . . 7,58

Stuhl, grünlich schleimig . . . . 4,66

$7^{\mathrm{h}}$ nachm. Urin . . . . . . . . . . . 7,50

Stuhl, wie vor. . . . . . 4,54

23. I. 9h vorm. Urin . . . . . . . . . . . 7,72

Stuhl, wie vor. . . . . . . 4,96

$5^{\text {h }}$ nachm. Stuhl, wie vor. . . . . . . . 5,01 
24. I. 9h vorm. Urin . . . . . . . . 8,26

Stuhl, gelblichgrüne Paste . . . 4,60

25. I. $9^{\mathrm{h}}$ vorm. Urin . . . . . . . . . 8,40

Stuhl, wie vor. . . . . . . 4,89

$5^{\text {h }}$ nachm. Urin . . . . . . . . . . . . 7,98

Stuhl, wie vor. . . . . . 4,82

Acidität des Blutes.

19. I. $9^{\text {n }}$ vorm. (am Anfang des Hungers)

Blut, aktuelle Reaktion ... 7,57

21. I. $9^{\mathrm{h}}$ vorm. (am Ende des Hungers)

Blut, aktuelle Reaktion... . . 7,33

\section{Versuch III.}

Hungerversuch von $2^{1 / 2}$ Tagen Dauer (mit $1 / 2$ Ringerlösung $+1 / 2$ Tee). Vom 27. I. $1511^{\mathrm{h}}$ nachm. bis 30 . I. $159^{\mathrm{h}}$ vorm. bekam das Kind nur obige Mischung zum Trinken, und zwar am 28. und 29. je $480 \mathrm{~g}$, am 30. bis zum Ende des Versuches $150 \mathrm{~g}$. Nahrung vor und nach dem Versuch $480 \mathrm{~g}$ Frauenmilch. In der Gewichtskurve trat während oder nach dem Versuche keine besondere Veränderung auf. Am 27. I. Gewicht $2340 \mathrm{~g}$, am 30. I. $2360 \mathrm{~g}$. Die Temperatur blieb unverändert um 36,6 bis $37^{\circ}$. D̉as Kind trank die Mischung wieder sehr gut, war matt gegen Ende des Versuches.

\section{Acidität des Stuhls und des Urins.}

Vor dem Hunger (diese Bestimmungen schließen sich den obigen, unmittelbar vorangehenden, an):

26. I. $g^{\mathrm{h}}$ vorm. Urin . . . . . . . 7,20

Stuhl, grünlich, sohleimig . . . 4,56

$5^{\mathrm{h}}$ nachm. Urin . . . . . . . . 7,84

27. I. $5^{\text {h }}$ nachm. Urin . . . . . . . . . . 7,53

Stuhl, gelbgrün, schleimig . . . 4,93

Während des Hungers:

28. I. $6^{\text {h }}$ vorm. Urin . . . . . . . . . . . . 7,19

Stuhl, gelbe, wenig grüne Paste . 4,82

$4^{\text {h }}$ nachm. Urin . . . . . . . . . . 6,85

29. I. 6 ${ }^{\text {h }}$ vorm. Urin, Polyurie ...... . . 5,63

Stuhl, bräunlichgelbe Salbe . . 6,31

$4^{\text {h }}$ nachm. Urin, Polyurie . . . . . . . 5,68

30. I. $6^{\text {h }}$ vorm. Urin, Polyurie . . . . . . . . 5,67

Von $9^{\text {h }}$ vorm. áb wieder Nahrung:

30. I. $4^{\mathrm{h}}$ nachm. Urin . . . . . . . . . . . . 5,95

Stuhl, dunkelbraun, zäh . . : 6,51

31. I. $6^{\text {h }}$ vorm. Urin, keine Polyurie mehr . . . 7,47

Stuhl, gelblichgrünliche Salbe . . 4,84

1. II. $6^{\mathrm{h}}$ vorm. Urin . . . . . . . . . . . . . 7,69

Stuhl, wie vor . . . . . 4, 48 
3. II. $8^{\mathrm{h}}$ vorm. Urin . . . . . . . . . . 8,11

Stuhl, gelbe, zähe Salbe . . . 4,85

4. II. $8^{\text {h }}$ vorm. Urin . . . . . . . . . 7,06

Stuhl, wie vor . . . . . . . 4,63

5. II. $8^{\mathrm{h}}$ vorm. Urin . . . . . . . . . . . . 6,78

Stuhl, gelb, wässerig, flockig . . 4,91

6. II. $8^{\text {h }}$ vorm. Urin . . . . . . . . . . . 7,10

Stuhl, wie vor . . . . . . 5,04

7. II. $8^{\mathrm{h}}$ vorm. Urin . . . . . . . . . . . . . 6,67

Stuhl, wie vor ....... . $\mathbf{4 , 9 6}$

8. II. $8^{\text {h }}$ vorm. Urin . . . . . . . . . . 7,01

9. II. $9^{\text {h }}$ vorm. Urin . . . . . . . . . 6,58

Stuhl: grünlichgelb, zäh . . . . 4,93

10. II. $9^{\mathrm{h}}$ vorm. Urin . . . . . . . . . . . . 7,49

Stuhl, wie vor . . . . . . 4, 4,94

11. II. $9^{\mathrm{h}}$ vorm. Urin . . . . . . . . . . . . . 7,37

Acidität des BIutes.

28. I. $8^{\text {h }}$ vorm. (am Anfang des Hungers)

COn-Regul.Breite

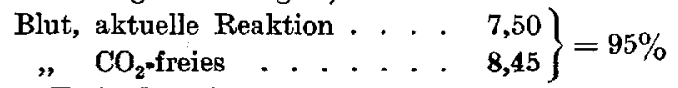

30. I. $9^{\mathrm{h}}$ vorm. (am Ende des Hungers)

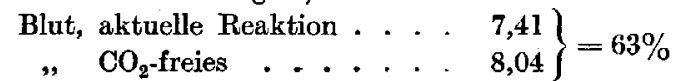

2. II. $12^{\mathrm{h}}$ mitt. (3 Tage nach dem Versuch)

Blut, aktuelle Reaktion . . . 7,50

Versuch IV.

Hungerversuch: Absoluter Hunger von $20 \mathrm{Stunden,} \mathrm{vom} \mathrm{14.} \mathrm{II.} 151^{\mathrm{b}}$ abends bis 15. II. $7^{\mathrm{h}}$ abends. Die Nahrung bestand vor- und nachher aus $480 \mathrm{~g}$ Frauenmilch. Das Gewicht sank während des Versuches von $2520 \mathrm{~g}$ auf $2440 \mathrm{~g}$ herab. Das Kind war anfangs sehr unruhig, gegen Ende des Versuches matt. Temperatur unverändert um 36,8 bis $37,1^{\circ}$.

I. Stuhl und Urin.

Vor dem Hunger (diese Bestimmungen schließen sich den obigen unmittelbar vorangehenden an):

12. II. $9^{\mathrm{h}}$ vorm. Urin . . . . . . . . . . . 6,78

Stuhl, gelb, grobflockig, leicht schlei-

mig . . . . . . . . . 4,91

13. II. $9^{\text {h }}$ vorm. Urin . . . . . . . . . . . . . 6,99

14. II. $\mathrm{g}^{\mathrm{h}}$ vorm. Urin . . . . . . . . . . . . . 6,69

Stuhl . . . . . . . . . 4,91

Während des Hungers:

15. II. $6^{\mathrm{h}}$ vorm. Urin . . . . . . . 7,08

$12^{\mathrm{h}}$ mitt. Urin . . . . . . . . . 6,58

$6^{\text {h }}$ nachm. Urin . . . . . . . . 5,94 
Vom 15. II. 7h nachm. wieder Nahrung:

16. II. $6^{\text {h }}$ vorm. Urin ........... 5, 50

Stnhl, dicke, gelbliche Paste . . 5,50

$5^{\mathrm{h}}$ nachm. Urin . . . . . . . . . . 6,29

Stuhl, wie vor . . . . . . . 5, 5,14

17. II. $9^{\text {h }}$ vorm. Urin . . . . . . . . . . 7,33

Stuhl, wie vor, nur mehr Schleim 4,85

II. Blut.

15. II. $8^{\mathrm{h}}$ vorm. (am Anfang des Hungers)

Blut, aktuelle Reaktion . . . 7,55 $\}=90 \%$

7h nachm. (am Ende des Hungers)

" $\mathrm{CO}_{2}$-freies ...... 8,45$\}=90 \%$

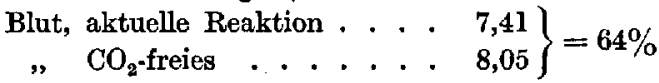

Die Veränderungen des Stuhls und des Urins in den vorangehenden drei Hungerversuchen sind auf Fig. 17 S. 317 und 19 S. 318 graphisch dargestellt. Die Veränderungen des Blutes während des 2. und 3. Hungerversuches (Versuch III und IV) sind auf Fig. 20, II graphisch dargestellt.

Bei demselben Kinde wurde direkt anschließend an die obigen Versuche eine Nahrungsveränderung von Frauenmilch auf $1 / 2-M i l c h$ (Larosan) vorgenommen, am 23. Il. $10^{\text {h }}$ morgens anfangend.

Versuch V, Stuhl: und Urinacidität bei Übergang von Frauenmilch auf $1 / 2-$ Milch

(Larosan):

18. II. (siehe die Werte an don vorhergehenden Tagen

im Versuch IV bei diesem Kinde).

18. II. 9h vorm. Urin ............ . 7,47

Stuhl, gelb, grobflockig, salbig . 4,97

21. II. $8^{\mathrm{h}}$ vorm. Urin . . . . . . . . . . . . $\mathbf{7 , 7 2}$

22. II. $8^{\mathrm{h}}$ vorm. Urin .......... . 7,53

23. II. $8^{\text {h }}$ vorm. Urin . . . . . . . . . 7,51

Von $10^{\text {h }}$ vorm. an $1 / 2^{-M i l c h ~(L a r o s a n) ~ m i t ~} 5 \%$ Rohrzucker:

$5^{\mathrm{h}}$ nachm. Urin . . . . . . . . 7,85

Stuhl, schleimig, gelblich, porös . 4,91

24. II. $8^{\text {h }}$ vorm. Urin . . . . . . . . . 6,52

Stuhl, gelbliche Salbe, kein Schlein $\quad \mathbf{6 , 9 7}$

$5^{\mathrm{h}}$ nachm. Urin . . . . . . . . . 6,18

Stuhl, braune knollige Salbe . . 7,47

25. II. $8^{\text {h }}$ vorm. Urin . . . . . . . . . . 6,36

Stuhl . . . . . . . . . . 8,13

26. II. von $10^{\text {h }}$ vorm. ab nur $1 / 2$-Milch mit $5 \%$ Rohrzucker ohne Larosanzusatz:

$10^{\mathrm{h}}$ vorm. Urin . . . . . . . . . . 6,22

Stuhl, wie vor . . . . . 8,13

$5^{\mathrm{h}}$ nachm. Urin . . . . . . . . . . . 7,19 


\begin{tabular}{|c|c|c|c|c|c|c|}
\hline II. & m. & Urin . . . . . & $\dot{0} \cdot \cdot$ & $\cdots$ & . & \\
\hline & & uhl, weißliche & Paste & & • & \\
\hline & $5^{\text {b }}$ nachm. & Urin & & & . & 10 \\
\hline II. & $8^{\mathrm{h}}$ & Urin & & & &, 25 \\
\hline & & Stuhl, wie vor & & & & 81 \\
\hline 1. III. & $8^{b}$ & Urin $\ldots$. & . & & . & 6,41 \\
\hline & & $U_{\mathbf{r}}$ & & & & 7,63 \\
\hline & & I, gelbbraun, & , brüchig & & & 7,85 \\
\hline 2. III & $8^{h}$ & $\mathrm{Ur}$ & . & . & . & 6,11 \\
\hline & & l, wie vor & & & & 7,53 \\
\hline & & Urin & & & & 6,43 \\
\hline & & $\mathrm{Ur}$ & & & & 6,12 \\
\hline & $\mathrm{rm}$ & Stuhl, wie vor & & & & 7,3 \\
\hline
\end{tabular}

Diese großen Veränderungen in der Acidität des Stuhls und Urins beim Nahrungswechsel sind auf Fig. 2 S. 297 graphisch dargestellt.

Fall Nr. 63.

Förster, Karl, Frühgeburt, hauptsächlich mit Kuhmilch ernäbrt. Geb. 4. IX. $14 \mathrm{im}$ ca. 7. Sohwangerschaftsmonate, angeblich im Anschluß an die Reposition eines Bauchbruches bei der Mutter. Gewicht ca. $1500 \mathrm{~g}$. Wird mit Kuhmilchmischungen von Anfang an ernährt. Gedeiht dabei schlecht, nimmt langsam ab und wird deswegen im Alter von ca. 2 Mon. am 28. X. 14 in die Anstalt eingeliefert.

28. X. 14. Status: Stark abgemagerte, wasserverarmte Frühgeburt, Gewicht $1330 \mathrm{~g}$, Länge $41 \mathrm{~cm}$. Trotz der starken Atrophie macht das Kind einen verhältnismäBig lebhaften und lebenskräftigen Eindruck. Bekommt bei uns ziemlich ausschließlich Frauenmilch, von $300 \mathrm{~g}$ bis $400 \mathrm{~g}$ langsam steigend bis zum 9 . XII., dann versuchshalber $1 / 2$-Milch (Larosan) mit $4 \%$ Soxhletzucker, später $1 / 2$-Milch mit etwas Beikost. Das Kind entwickelt sich dabei recht gut, nimmt regelmäßig zu, bleibt von allen Infektionen versehont und wird erst im Alter von 8 Mon. am 4. V. 15 mit sinem Gewicht von $4200 \mathrm{~g}$ gesund entlassen.

Bei dem Kinde wurden verschiedene Untersuchungen vorgenommen.

Die Protokolle der Hungerversuche bringe ich zunächst:

I. Hungervers uch (mit $1 / 2$ Ringerlōsung $+1 / 2$ Tee):

Dauer des Hungers ca. 21/2 Tage, vom 18. I. $1511^{\mathrm{h}}$ nachm. bis 21 . I. 15 $9^{\mathrm{b}}$ vorm. Die Nahrung bestand vor dem Hunger aus $540 \mathrm{~g} 1 / 2$-Milch (Larosan) mit $4 \%$ Soxhletzucker, nach dem Hunger einen Tag lang aus derselben Menge Frauenmilch, später wieder aus derselben Menge 1/2-Milch (Larosan) wie vordem. Das Kind bekam am 1. und 2. Tage des Versuches je $250 \mathrm{~g}$ obigen Gemisches, am 3. Tage kis zum Ende des Versuches 200 g. Das Gewicht sank während des Versuches von $2740 \mathrm{~g}$ auf $2640 \mathrm{~g}$ herab. Die Temperatur blieb unbeeinflußt $36,8^{\circ}$ bis $37^{\circ}$. Am 1. Tage war das Kind unruhig, am 2. Tage wieder ruhiger, machte etwas matten Eindruck gegen Ende des Versuches.

I. Stuhl und Urin.

Vor dem Hunger:

16. I. $8^{\mathrm{h}}$ vorm. Urin . . . . . . . . . 6,62

18. I. $8^{\mathrm{h}}$ vorm. Urin ........, 6,59 


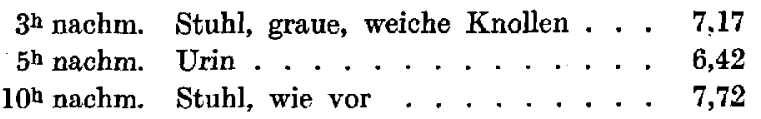

Während des Hungers;

19. I. $6^{\text {h }}$ vorm. Urin . . . . . . . . . 6,48

$5^{\text {h }}$ nachm. Urin . . . . . . . . . . . 5,64

Stuhl, wie vor . . . . . . 7,28

20. I. 9 vorm. Urin ............ 5,49

$5^{\mathrm{h}}$ nachm. Urin . . . . . . . . . . 5, 57

21. I. $9^{\text {h }}$ vorm. Urin . . . . . . . . . . 5,52

Von $9^{\mathrm{h}} \mathrm{ab}$ wieder Nahrung:

21. I. $5^{\text {h }}$ nachm. Urin . . . . . . . . . . . . . 6,02

22. I. $9^{\mathrm{h}}$ rorm. Urin . . . . . . . . . . . . . 7,59

$5^{\mathrm{b}}$ nachm. Urin . . . . . . . . . . 7,91

23. I. $9^{\text {h }}$ vorm. Urin . . . . . . . . . . . 7,79

9h vorm. Stubl, bräunlich, brüchig (seit dem

Hunger verstopft gewesen) . . . $\mathbf{7 , 4 3}$

$5^{\mathrm{n}}$ nachm. Urin . . . . . . . . . . . . . 7,27

24. I. 9h vorm. Urin . . . . . . . . . . . . . . 6,88

25. I. 9h vorm. Urin . . . . . . . . . . 5,67

Stuhl, weißlich, brüchig . . . 7,32

$5^{\mathrm{h}}$ nachm. Urin . . . . . . . . . . . 6, 6,25

II. Blut.

19. I. $9^{\mathrm{h}}$ porm. (am Anfang des Hungers)

$\mathrm{CO}_{\mathbf{2}}$-Regul. Breite

$\left.\begin{array}{c}\text { Blut, aktuelle Reaktion . . . . . } \\ \text {, } \quad \mathrm{CO}_{2} \text {-freies . . . . . . } 8,40\end{array}\right\}=87 \%$

21. I. $9^{\mathrm{n}}$ vorm. (am Ende des Hungers)

$\left.\begin{array}{c}\text { Blut, altuelle Reaktion . . . . } \\ \text {, } \quad \mathrm{CO}_{2} \text {-freies . . . . . . } 73,98\end{array}\right\}=45 \%$

II. H u ngerversuch (mit. $1 / 2$ Ringerlösung $+1 / 2$ Tee):

Dauer des Hungers ca. 21/2 Tage, vom 27 . I. $1511^{\mathrm{h}}$ nachm. bis 30 . I. 15 $9^{\text {h }}$ vorm. Die Nahrung bestand vor und nach dem Hunger aus $540 \mathrm{~g} 1 / \mathrm{g}^{-M i l c h}$ (Larosan) mit 4\% Soxhletzucker. Das Kind bekam am 1. und 2. Tage des Versuches je $250 \mathrm{~g}$ obigen Gemisches, am 3. Tage bis zum Ende des Versuches $185 \mathrm{~g}$. Während des Versuches sank das Gewicht von $2820 \mathrm{~g}$ bis $2680 \mathrm{~g}$. Die Temperatur hielt sich während des Versuches konstant bei $37^{\circ}$, dagegen war sie am 30. I. abends plötzlich bis auf $38,2^{\circ}$ erhöht, am folgenden Morgen aber wieder $36,8^{\circ}$. Vor der Temperatursteigerung hatte das Kind schon 3 Flaschen zu je 90 g obiger Nahrung bekommen. Es trank die Mischung ganz gut, war am Anfang des Versuches unruhig, wurde gegen Ende des Versuches etwas ruhiger.

\section{Stuhl und Urin.}

Vor dem Hunger (diese Periode schließt sich unmittelbar an die vorher angegebenen

Untersuchungen an):

26. I. $9^{\mathrm{n}}$ vorm. Urin . . . . . . . . . . 5,60

Stuhl, gelblichweiß, salbig . . . 6,49 
$5^{\mathrm{h}}$ nachm. Urin . . . . . . . . . 6 6,45

27. I. $9^{\mathrm{h}}$ vorm. Urin ........... 5,76

Stuhl, wie vor . . . . . . 6,4l

Während des Hungers:

28. I. $6^{\text {h }}$ rorm. Urin . . . . . . . . 7,03

Stuhl, gelblichweiß, pastig . . . $\mathbf{7 , 2 3}$

$4^{\mathrm{h}}$ nachm. Urin . . . . . . . . 5,86

Stuhl, wie vor . . . . . . . 7,48

29. I. $6^{\mathrm{h}}$ vorm. Urin . . . . . . . . . 5,47

$4^{\mathrm{h}}$ nachm. Urin . . . . . . . . . 5,80

30. I. $6^{\mathrm{h}}$ vorm. Urin, gelblich (keine Polyurie) . 5,76

Von $9^{\text {h }}$ vorm. an wieder Nahrung:

30. I. $4^{\text {h }}$ nachm. Urin . . . . . . . . . . . 6,17

31. I. $6^{\text {h }}$ vorm. Urin . . . . . . . . . 7,61

$4^{\mathrm{h}}$ nachm. Urin . . . . . . . . . . . 6,96

Stuhl, gelblichweiß, brüchig (seit

Anfang des Hungers verstopft) 7,72

1. II. $6^{\text {h }}$ vorm. Urin . . . . . . . . . . . 6,44

Stuhl, wie vor . . . . . . 7,98

2. II. $6^{\text {h }}$ vorm. Urin . . . . . . . . . . 6,90

Stuhl . . . . . . . . . . 7,88

3. II. $6^{\mathrm{h}}$ vorm. Urin . . . . . . . . . . 5,96

Stuhl, weißlich, brüchig . . . 7,73

4. II. $6^{\text {h }}$ vorm. Urin . . . . . . . . . . 5,99

Stuhl, wie vor . . . . . . . $\mathbf{8 , 0 9}$

5. II. $6^{\text {h }}$ vorm. Urin . . . . . . . . . . . . . 5,52

Stuhl, wie vor . . . . . . . 8,01

6. II. $6^{\text {h }}$ vorm. Urin . . . . . . . . . . . . . 6,28

Stuhl, bräunlich, brüchig . . . . $\mathbf{7 , 8 2}$

7. II. $6^{\text {h }}$ vorm. Urin . . . . . . . . . . . 6.52

Stuhl, wie vor . . . . . . . 7,72

8. II. $6^{\text {h }}$ vorm. Urin . . . . . . . . . . . . . 6,28

Stuhl, wie vor....... 8,00

II. BIut.

28. I. $8^{\text {h }}$ vorm. (am Anfange des Hungers)

$\mathrm{CO}_{2}-$ Regul. Breite

Blut, aktuelle Reaktion . . . . 7,46

, $\mathrm{CO}_{2}$-freies

$8,46\}=104 \%$

30. I. $9^{\text {h }}$ vorm. (am Ende des Hungers)

Blut, aktuelle Reaktion . . . . 7,47)

, $\mathrm{CO}_{2}$-freies ...... 8,13

$\left.\begin{array}{l}7,47 \\ 8,13\end{array}\right\}=66 \%$

III. Hungerversuch (20stündiger absoluter Hunger):

Am 14. II. um $9^{\mathrm{h}}$ abends die letzte Mahlzeit, $90 \mathrm{~g} 1 / 2$-Milch (Larosan) mit $5 \%$ Rohrzucker. Dann nichts zum Trinken bis 15 . II. $11^{\text {h }}$ nachm. Von obiger Nahrung wurden vor und nach dem Hunger $540 \mathrm{~g}$ täglich gereicht. Die Temperatur in den Tagen vor und nach dem Hungerversuch war vollkommen mono- 
therm $37^{\circ}$; nach 18 stündigem Hunger stieg die Temperatur langsam bis $37,8^{\circ}$, hielt sich in dieser Höhe ca. 12 Stunden lang (trotz schon eingereichter Nahrung) und fiel dann im Verlaufe der 4 nächsten Stunden wieder auf $37^{\circ}$ zurück. Das Kind machte gegen Ende des Hungers etwas müden Eindruck. Das Gewicht sank vom 14. II. bis 15. II. von $3000 \mathrm{~g}$ auf $2900 \mathrm{~g}$ zurück.

\section{Stuhl und Urin.}

Vor dem Hunger (diese Periode schließt sich mit ihren Bestimmungen direkt an die vorhergehenden an):
9. II. $8^{\text {h }}$ vorm.
Urin . . . . . . . . . 5,96
Stuhl, weißlichgelb, brüchig . . . 7,94
10. II. $8^{\mathrm{h}}$ vorm.
Urin . . . . . . . . 6, 6,29
11. II. $8^{\text {h }}$ vorm.
Stuhl, wie vor . . . . . . . 8,40
12. II. $8^{\text {h }}$ vorm.
Urin . . . . . . . 5,92
Stuhl, wie vor . . . . . . 7,98
13. II. $8^{\text {h }}$ vorm.
Urin . . . . . . . . . 6,30
Stuhl, wie vor ..... 7,57
14. II. $8^{\text {h }}$ vorm. Urin . . . . . . . . . . 6,03
Stuhl, gelbliche Salbe . . . . 6,98

Während des Hungers:

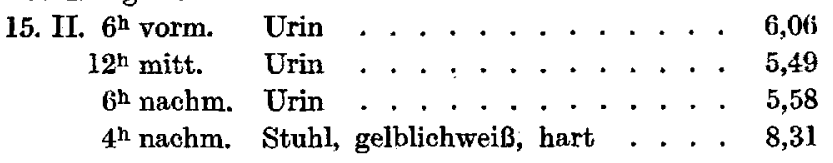

Von $7^{\text {h }}$ nachm. ab wieder Nahrung 1/2-Milch (Larosan) mit $5 \%$ Rohrzucker:

16. II. $6^{\text {h }}$ vorm. Urin ........... 5,69

Stuhl, wie vor . . . . . . . 8,31

$5^{\mathrm{h}}$ nachm. Urin ........... 5,89

17. II. $9^{\text {h }}$ vorm. Urin . . . . . . . . . . . . 6,55

18. II. $9^{\mathrm{h}}$ vorm. Urin ... . . . . . . . . 7,11

$5^{\mathrm{h}}$ nachm. Urin . . . . . . . . . . 6,57

\section{Blut.}

15. II. $8^{\text {h }}$ vorm. (am Anfang des Hungers)

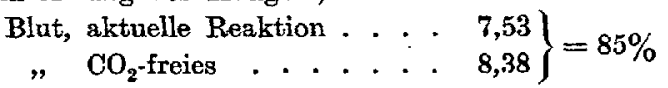

$7^{\text {h }}$ nachm. (am Ende des Hungers)

$\left.\begin{array}{c}\text { Blut, aktuelle Reaktion . . . . } \\ \text { " } \mathrm{CO}_{2} \text {-freies . . . . . } 76,92\end{array}\right\}=46 \%$

Bei demselben Kinde wurde anschließend an die obigen Versuche eine Nahrungsveränderung von $1 / 2$ Milch (Larosan) auf Frauenmilch, $540 \mathrm{~g}$ täglich, forgenommen, und zwar am 19. II. $6^{\mathrm{h}}$ früh Übergang.

IV. Versuch: Stuhl und Urin bei Utbergang in die Frauenmilchernährung.

19. II. (siehe die Werte an den vorhergehenden Tagen im III. Hungerversuch).

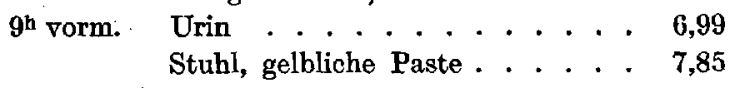


$4^{\mathrm{h}}$ nachm. Urin . . . . . . . . . 6,98

Stuhl, weißliche Salbe . . . 7,09

20. II. $9^{\mathrm{h}}$ vorm. Urin . . . . . . . . . . . 6,49

Stuhl, weiche, gelbe Salbe . . . 4,68

$5^{\text {h }}$ nachm. Urin . . . . . . . . 6,75

21. II. $8^{\mathrm{h}}$ vorm. Urin . . . . . . . . . . . . 7,35

Stuhl, gelbliche poröse, leicht grün-

liche Masse . . . . . . 4,82

$5^{\text {h }}$ nachm. Urin . . . . . . . . . 7,31

22. II. $8^{\text {b }}$ vorm. Urin . . . . . . . . . . . 7,53

Stuhl, wie vor . . . . . 4,90

$5^{\mathrm{h}}$ nachm. Urin . . . . . . . . . . 7,58

23. II. $9^{\mathrm{h}}$ vorm. Urin . . . . . . . . . . . . . 7,79

Stuhl, wie vor . . . . . . 4,54

$5^{\mathrm{h}}$ nachm. Urin . . . . . . . . . . . $\mathbf{7 , 4 6}$

24. II. $9^{\text {h }}$ vorm. . Urin . . . . . . . . . 7,86

$5^{\text {h }}$ nachm. Stuhl, grün, schleimig ... . 5,15

25. II. $8^{\mathrm{h}}$ vorm. Urin . . . . . . . . . . 7,40

Stuhl, schleimig, grün . . . . . 4,68

26. II. $8^{\text {h }}$ vorm. Urin . . . . . . . . . . . . . 7,92

Stuhl, wie vor . . . . . 4,42

27. II. $7^{\mathrm{h}}$ vorm. Urin . . . . . . . . . . 7,85

Stuhl, grün, schleimig, zäh . . 4,80

28. II. $8^{\mathrm{h}}$ vorm. Urin ........... . 7,21

Stuhl, wie vor . . . . . . . 4,67

V. Versuch (Übergang am 28. II. $1^{\text {h }}$ mittags von Frauenmilch auf $1 / 2$-Milch mit 5\% Rohrzucker):
1. III. $8^{\text {h }}$ vorm. Urin . . . . . . . . . . 6,49
$5^{\mathrm{h}}$ nachm. Urin . . . . . . . . 6,65
Stuhl, gelbliche Salbe . . . . 5,89
2. III. $8^{\mathrm{h}}$ vorm. Urin . . . . . . . . . . 6,56
Stuhl, gelbliche, dicke Paste . . 7,69
$5^{\text {h }}$ nachm. Urin . . . . . . . 6 6,85
Stuhl, wie vor ....... 7,27
3. III. $5^{\mathrm{h}}$ nachm. Urin . . . . . . . . . . 6,46
4. III. $8^{\text {h }}$ vorm. Stuhl, gelbliche Paste . . . . 7,48

Die Veränderungen in der Acidität des Stuhls und des Urins während des I. und II. Hungerversuches sind auf Fig. $18 \mathrm{~S} .317$, die Veränderungen während des III. Hungerversuches und während der Nahrungsveränderungen (Versuch IV und V) sind auf Fig. 4 S. 298, das Verhalten des Blutes im II. und III. Hungerversuch ist auf Fig. 20, I S. 318 graphisch dargestellt.

Außerdem wurden bei diesem Kinde Versuche über den

Einfluß der Acidität der Nahrung a uf die Acidität des Urins angestellt. 
I. Versuch: $\frac{\text { Essigsäure }}{\text { Na-Acetat }}+$ Frauenmilch.

Vom 28. XI. $9^{\text {h }}$ nachm. bis 2. XII. $6^{\text {h }}$ vorm. bekam das Kind täglich $400 \mathrm{~g}$ von folgendem saurem Nahrungsgemisch:

$$
\begin{aligned}
& \text { von } \frac{\text { n-Essigsäure } 1 \text { Teil }}{n \text {-Na-Acetat } 1 \text { Teil }}=10 \mathrm{ccm} \\
& \text { und Frauenmilch } \quad=100,0 \mathrm{ccm}
\end{aligned}
$$

$P_{H}$ dieses Gemisches war 4,42 (die der reinen Frauenmilch ist ca. 6,80).

Das Kind trank die Mischung gern, nahm während des Versuches von $1800 \mathrm{~g}$ bis $1920 \mathrm{~g} \mathrm{zu}$. Diese Zunahme bewegte sich in normalen Grenzen.

$$
P_{\text {H }} \text { des Urins. }
$$

28. XI. $8^{\text {h }}$ vorm. vor der Säuremiloh . . . : 6,49

29. XI. $8^{\text {h }}$ vorm. während der Säuremilch . . 7,52

$8^{\mathrm{h}}$ nachm. " $\quad$ " . . . 7,25

30. XI. $8^{\text {h }}$ vorm. $\quad$ " $\quad$ " $\quad$, . 8,55

$8^{\text {h }}$ nachm. $\quad, \quad$, $\quad$, . . 8,40

-1. XII. $8^{\text {h }}$ vorm. $\quad " \quad, \quad, \quad$. . 8,25

$8^{\text {h }}$ nachm. " . " , , $\quad . .7,55$

2. XII. $6^{\text {h }}$ vorm. wieder nur Frauenmilch:

$8^{\text {h }}$ vorm. . . . . . . . . 8,07

3. XII. $8^{\mathrm{h}}$ vorm. $\quad . \quad \ldots . . . . . . . .7,97$

$8^{\text {h }}$ nachm. . . . . . . . . . . . 8,42

4. XII. $8^{\mathrm{h}}$ vorm. . . . . . . . . . . . . 7,43

$5^{\mathrm{h}}$ nachm. . . . . . . . . . . . 7,90

5. XII. $8^{\mathrm{h}}$ vorm. . . . . . . . . . . 7,40

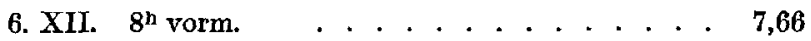

7. XII. $8^{\text {h }}$ vorm. . . . . . . . . . . . . 7,06

$7^{\mathrm{h}}$ nachm. . . . . . . . . . . . . 6,94

8. XII, $10^{\mathrm{h}}$ vorm. . . . . . . . . . . 6,70

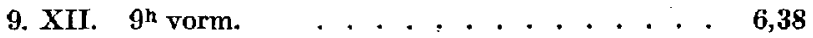

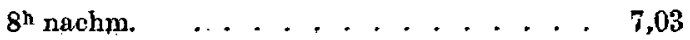

10. XII. $9^{\mathrm{h}}$ vorm. . . . . . . . . . . 6,85

$8^{\mathrm{h}}$ nachm. . . . . . . . . 6 6,65

11. XII. $8^{\mathrm{h}}$ vorm. . . . . . . . . . . 6,35

II. Versuch: $\frac{\text { Milchsäure }}{\text { Na-Lactat }}+$ Frauenmileh.

Vom 12. XII. $6^{\text {h }}$ früh bis 14. XII. $6^{\text {h }}$ früh bekam das Kind täglich ca. $400 \mathrm{~g}$ von folgendem saurem Nahrungsgemisch:

$$
\begin{aligned}
\operatorname{von} \frac{\mathrm{n} \text {-Milchsäure } 1 \text { Teil }}{\mathrm{n} / \mathrm{2} \text {-Na-Lactat } 4 \text { Teile }} & =10,0 \mathrm{~cm} \\
\text { und Frauenmilch } & =100,0 \mathrm{ccm}
\end{aligned}
$$


$\mathrm{P}_{\mathbf{H}}$ dieses Gemisches war 4,58. Das Casein fiel hier wie in der obigen Mischung in feinen Flocken aus. Das Kind trank die Mischung ganz gern. Die Gewichtskurve zeigte während des Versuches den normalen Verlauf. Gewicht am 12. XII. $2080 \mathrm{~g}$, am 14. XII. $2140 \mathrm{~g}$.

\section{$\mathbf{P}_{\mathbf{H}}$ des Urins.}

12. XII. $7^{\mathrm{h}}$ vorm. $\ldots . . . . . .6,68$

$4^{\mathrm{h}}$ nachm. . . . . . . . . . . 7,13

13. XII. 9h vorm. Polyurie . . . . . . . 8,25

$7^{\mathrm{h}}$ nachm. Polyurie . . . . . . . 8,88

14. XII. $7^{\mathrm{h}}$ vorm. Polyurie . . . . . . . . 8,87

$6^{\text {h }}$ vorm. ab wieder Frauenmilch

$5^{\text {h }}$ nachm. Polyurie . . . . . . . . 8,12

15. XII. 9h vorm. Polyurie ........ . 8,44

$5^{\text {h }}$ nachm. Polyurie . . . . . . . 7,53

16. XII. 9h vorm. Polyurie . . . . . . . . . 7,52

$8^{\mathrm{h}}$ nachm. Polyurie . . . . . . . . 7,49

17. XII. 9h vorm. Polyurie . . . . . . ${ }^{\circ} 7,63$

$5^{\mathrm{h}}$ nachm. Polyurie . . . . . . 7,34

Vom 17. XII. bis 20. XII. neben Frauenmilch ca. $200 \mathrm{~g}$ Buttermileh täglich.

18. XII. $9^{\mathrm{h}}$ vorm. . . . . . . . . . . . . . 6,83

$6^{\mathrm{h}}$ nachm. . . . . . . . . . . 7,05

19. XII. $10^{\mathrm{h}}$ vorm. . . . . . . . . . . . . 6,49

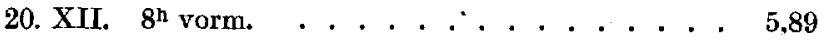

21. XII. $8^{\mathrm{h}}$ vorm. . . . . . . . . . . . . 6,39

$8^{\mathrm{h}}$ nachm. . . . . . . . . . . 6,22

22. XIr. $8^{\mathrm{h}}$ vorm. . . . . . . . . . . . 5,97

Diese beiden Versuche mit sauren Nahrungsgemischen sind auf der Fig. 6 S. 302 graphisch dargestellt.

Außerdem wurde bei dem Kinde am I0. I. 15 ein weiterer Nahrungswechsel vorgenommen und die Verschiebungen der $P_{H}$ des Urins wie folgt bestimmt:

6. I. $8^{\text {b }}$ vorm. nur Frauenmilch täglich $420 \mathrm{~g}$. . . 6,82

8. I. $8^{\mathrm{h}}$ vorm. $, \quad, \quad$. . . . . . . 6, 6,79

9. 1. $8^{\mathrm{h}}$ vorm. Frauenmileh $+1 / \mathrm{g}^{\mathrm{A}}$-Milch $\overline{\mathrm{a}} \overline{\mathrm{a}} 210 \mathrm{~g} \cdot 6,85$

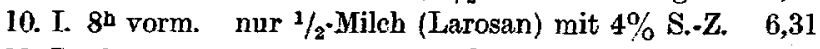

11. I. $8^{\text {h }}$ vorm. do. 6,51

12. I. $8^{\text {h }}$ vorm. do. 6,17

13. I. $8^{\text {h }}$ vorm. do. $\quad 5,62$

15. I. $\gamma^{\text {th }}$ vorm. do. $\quad 5,99$

16. I. $8^{\text {h }}$ vorm. do. 6,62

18. I. $8^{\text {h }}$ vorm. do. do 6,59

Aus dem Versuch ist klar ersichtlich, wie die Acidität des Urins bei Zufulur von Kuhmilch rapid zu steigen anfängt, um die größte Acidität nach Übergang in die volle Kuhmilchernährung zu erreichen. Nachher findet wieder ein lang. sames Sinken der Acidität trotz Kuhmilchernährung statt. Die Werte erreichen aber nicht die bei Frauenmilch gefundene Höhe. 
und Intoxikationsacidosis in ihren Beziehungen zueinander.

\section{Reaktion des Blutes und der Gewebe beim hungernden und normalen Tier.}

Acidität des Blutes und der Gewebe beim hungernden und normalen Tier.

Fall Nr. 64.

Siebentägiger Hungerversuch bei einem' kräftigen, ausgewachsenen Kaninchen.

Gewicht $2880 \mathrm{~g}$.

28. VI. $151^{\text {h }}$ nachm. Beginn des Hungers, Blutentnahme aus der Ohrvene.

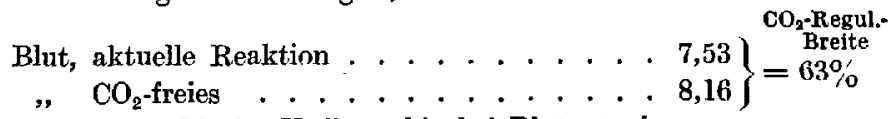

29. VI. Liegt meistens ruhig im Käfig, ruhig bei Blutentnahme.

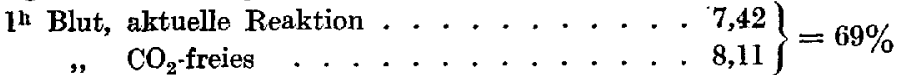

2. VII. Ruhig wie vor, links eiterige Conjunctivitis, sonst o. B.

$1^{\text {h }}$ Blut, aktuelle Reaktion . . . . . . . . 7,23

" $\mathrm{CO}_{2}$-freies ........... 7,82

5. VII. Sitzt immer in ein und derselben Ecke des Käfigs, wenn

man es stört, macht es nur ein paar Sprünge und bleibt

wieder sitzen.

$3^{\text {h }}$ nachm. Aus der Carotis verblutet, Sektion gleich danach.

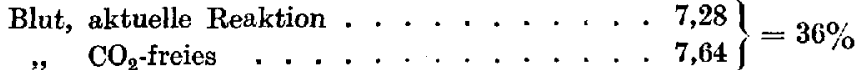

Gehirnsubstanz, ungekocht . . . . . . . 6,63

gekocht . . . . . . . . 6,84

1. Muskel, Pectoralis, ungekocht . . . . 6,67

gekocht . . . . . 6,79

2. Muskel, Tleopsoas, 1 Std. später herausgenommen, ungekocht . . . . . . . . . . 6,36

Herz, schlug noch beim Spalten, ungekocht . . . . . . . . . 6,63

gekocht . . . . . . . . . 6,65

Leber, ungekocht . . . . . . . . . 6,54

gekocht . . . . . . . . . . . 6,82

Galle, $4 \mathrm{ccm}$, grünlich, verhältnismäßig dünnflüssig 5,87

Milz, ungekocht . . . . . . . . . 6,55

Niere, ungekocht . . . . . . . . 6,52

gekocht . . . . . . . . . 6,84

Urin .............. . . 5,15

Beim Töten wog das Kaninchen $2300 \mathrm{~g}$. Sektion: Organe anscheinend alle gesund, Dünndarm leer, im Dickdarm noch dicke breiige Fäkalmassen.

Die vorstehenden Werte sind in der Fig. 21, I graphisch dargestellt.

Fall Nr. 65.

Acidität der Organe und des Blutes bei einem gesunden Kaninchen, ca. $2700 \mathrm{~g}$. 6. VII. Aus der Carotis verblutet, Sektion gleich danach.

$$
\begin{aligned}
& \text { Blut, aktuelle Reaktion . . . . . . . } 7,47)^{\substack{\mathrm{CO}_{2} \text {-Regul. } \\
\text { Breite }}} \\
& \text { " } \mathrm{CO}_{2} \text {-freies ...... . . . . . . } 8,03 \mathrm{~J}=56 \%
\end{aligned}
$$

Zeitschrlft für Kinderheilkunde. O. XIV. 
1. Muskel, Pectoralis. ungekocht . . ...... . 6,31

gekocht : . . . 6,74

2. Muskel, Ileopsoas, ca. I Std. : später herausgenommen, ungekocht . . . . . . 6,33

Herz, ungekocht . . . . . . . 6,47

gekocht . ... . . . . . 6,47

Leber, ungekocht . . . . . . . . . . 6,66

gekocht . . . . . . . . 6,66

Milz, ungekocht ......... 6,52

Niere, ungekocht .......... 6,61

gekocht . . . . . . . . . 6,74

Gehirn, ungekocht . . . . . . . . .. 6,74

gekocht . . . . . . . . . 6,71

Die vorstehenden. Werte sind auf Fig. 21, II S. 321 graphisch dargestellt.

Fall Nr. 66.

Zehntägiger Hungerversuch bei einem jüngeren Kaninchen, Gewicht $2080 \mathrm{~g}$. 6. VII. $2^{\mathrm{h}}$ mitt. Beginn des Hungers, Blutentnahme aus der Ohrvene.

10. VII. $2^{\text {h }}$ mitt.

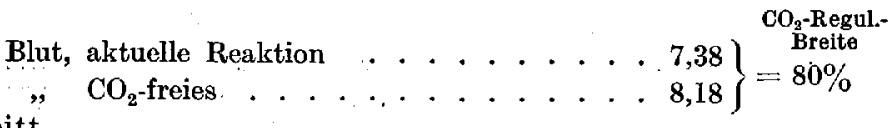

Blut, aktuelle Reaktion . . . . . . . 7,28

" $\mathrm{CO}_{2}$-freies ........ . 7,90$\}=62 \%$

12. VII. $2^{\text {h }}$ mitt. deutlich abgenagert, macht aber noch kräftige Sprünge beim Versuch es abzufangen, sonst ruhig im Käfig.

Blut, aktuelle Reaktion . . . . . . . 7,07

" $\mathrm{CO}_{2}$-freies .......... . 7,75$\}=68 \%$

16. VIII. $2^{\mathrm{h}}$ mitt. Äthernarkose, aus der Carotis verblutet, Sektion gleich danach.

Blut, aktuclle Reaktion ....... . 7,14 " $\mathrm{CO}_{2}$-freies .......... 7,57$\}=43 \%$

1. Muskel, vor dem Exitus das ganze Vorderbein in der Narkose mit Schere abgeschnitten, Extrakt gleich gemessen, ungekocht . . . 6,82 das Hinterbein wie vor, dann gleich in kochendes Wasser geworfen, gekocht . . . . . 6, 60

2. Muskel, ca. $20 \mathrm{Min}$. nach dem Tode das andere Hinterbein abgeschnitten, ein Teil der Muskulatur, ungekocht . . . . . . . 6,67 der andere Teil gekocht , . . . . . 6,77

Herz, schlug noch beim Spalten, tungekocht . . . . . . . . . 6,67 gekocht . . . . . . . . . . 6,69

Leber, ungekocht . . . . . . . . . 6,81 gekocht . . . . . . . . . 6,95

Galle, dunkelgrün und zäh . . . . . . . 6,14 
und Intoxikationsacidosis in ihren Beziehungen zueinander.

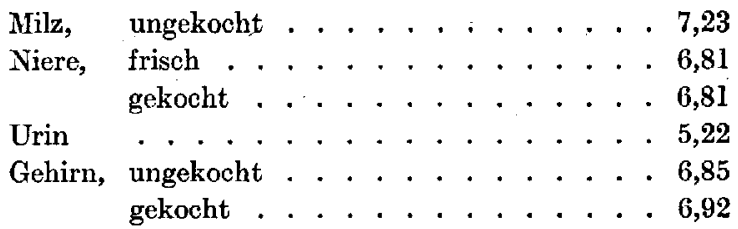

Die vorstehenden Werte sind auf Fig. 21, III S. 321 graphisch dargestellt.

Fall Nr. 67.

Gesundes ausgewachsenes Meerschweinchen, Gewicht $300 \mathrm{~g}$.

10. VII. $12^{\text {h }}$ mitt. Äthernarkose, Blut aus der Carotis.

Blut, aktuelle Reaktion . . . . . . . . . 7,30

" $\mathrm{CO}_{2}$-freies . . . . . . . . . . . . . 7,89

1. Muskel, linkes Hinterbein in der Narkose am. putiert, enthäutet, Extrakt gleich gemessen, ungekocht . . . . . . . . . 6,45 rechtes Hinterbein wie vor, dann in kochendes Wasser geworfen, gekocht . . . . . . 6,73

2. Muskel, ca. 20 Min. später das linke Vorderbein amputiert, ein Teil wurde zerrieben, der Extrakt stand 1 Std. lang, ungekocht . . . . 6,51 ein anderer Teil desselben Beines gekocht . . . 6,78 Leber, ungekocht . . . . . . . . . 6,84 gekocht .. . . . . . . . . . 7,11

Die vorstehenden Werte sind auf Fig. 10, II S. 309 graphisch dargestellt. Fall Nr. 68.

Gesundes Meerschweinchen, altes Tier, $320 \mathrm{~g}$.

9. VIII. Äthernarkose, Sektion während derselben, Blut aus der Carotis.

Blut, aktuelle Reaktion . . . . . . . . . 7,19 , $\mathrm{CO}_{2}$-freies ........... . 7,75

1. Muskel, Pectoralis, in der Narkose heraus. genommen, ungekocht. . . . . . : 6,36 gekocht . . . . . . . 6,66

Herz, schlug noch beim Spalten, ungekocht . . . . . . . . . . . . 6,44 gekocht . . . . . . . . . . 6,65

Leber, ungekocht . . . . . . . . . . . 6,66 gekocht . . . . . . . . 6,65

Galle, ockergelb, dünnflüssig . . . . . . 8,35

Milz, ungekocht . . . . . . . . . . . . 6,48

Niere, ungekocht . . . . . . . . . 6,53 gekocht . . . . . . . . . . 6,72

Die vorstehenden Werte sind auf Fig. 10, I S. 309 graphisch dargestellt. 


\title{
VII. Reaktion des Blutes einschließlich der $\mathrm{CO}_{2}$-Regulationsbreite und der $\mathrm{O}_{2}$-Dissoziationskurve bei Intoxikation. \\ (Phosphatkinder darunter!)
}

\author{
Fall Nr. 69.
}

Fischer, Rolf, 6 Wochen, schwere chronische Ernährungsstörung, Intoxikation, geb. 9. IV. 16, Geburtsgewicht $3750 \mathrm{~g}$, 10 Tage Brust, dann 1/3 Milch ohne Zucker, dabei starke Abnahme und schlechtes Gedeihen. Am 14. V. plötzlich dünne Stühle und Erbrechen, seit 17. bis 19. Eiweißmilch $6 \times 6^{\prime}$. Weil allmählich verfallen und bewußtlos geworden, am 19. V. zur Aufnahme gebracht. Gewicht $2980 \mathrm{~g}$, stark atrophisch, tief benommen, Blut und Eiter aus dem rechten Ohr, eiterige, stinkende Stühle. Frauenmilch und Tee in.kleinen Dosen mit Sonde. Am 20. V. noch schwer benommen, Untertemperatur, andauernd eiterige Stühle, subeutane Phosphatgemischinjektion um 1 Uhr, um 2 Uhr Exitus. Sektion gleich danach. Diagnose: Enteritis haemorrh. mit diphtheritischen Darmgeschwüren, Otitis media dextra.

Bei den am Leben und nach dem Tode vorgenommenen Untersuchungen wurden folgende $\mathrm{P}_{\mathrm{II}}$-Werte gefunden:

20. V. $12^{\mathrm{h}} 40^{\prime}$ Blutentnahme durch Sinuspunktion, Hirudin.

Blut, aktuelle Reaktion . . . . . . . . 7,17 )

, $\mathrm{CO}_{2}$-freies ........... 7,81

$\mathrm{CO}_{3}$-Regul.-

Lumbalpunktion: Liquor, klar . . . . . 7,81

Urin, frisch katheter., leicht trübe, Eiweiß, Zucker + 5,58

Stuhl, dünn wässerig, eiterig, flockig . . . 8,10

20. V. $1^{\text {h }}$ suboutane Einspritzung $120 \mathrm{com}$ Phosphatgemisch.

Resorption Jangsam, doch bis zum Exitus vollständig.

$2^{\text {h }}$ Exitus.

Herzpunktion und Sektion ca. 10 Min. später.

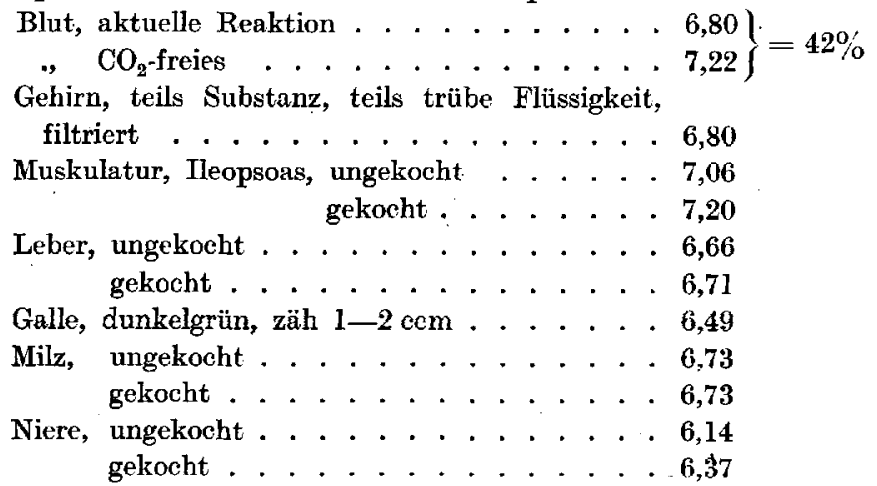

Inhalt des Magendarmkanals:

In der Magenschleimhaut kleine Blutungen, die Darmschleimhaut durch und durch hämorrhagisch verfärbt. Im untersten Ileum größere diphtheritische Darmgeschwüre, im Dickdarm ähnliche kleinere Geschwüre. 
Magen ziemlich leer, enthält nur einige Kubikzentimeter schleimige, mit eiterigen ( ?) Flocken vermischte Flüssigkeit

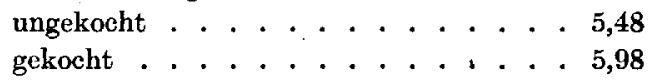

Duode $\mathrm{n} \mathrm{m}$, ca. $50 \mathrm{~cm}$ unterhalb Pylorus, braune

flockige wässerige Massen

ungekocht . . . . . . . . . . . 7,17

gekocht . . . . . . . . . . . . 7,86

Jejunum, eiterig, brüunlich, dünnbreiig

ungekocht . . . . . . . . . 7,48

gekocht . . . . . . . . . . . 8,04

Heum, dicht oberhalb Valvula Bauhinii, Inhalt

wie Jejunum

ungekocht . . . . . . . . . . . 7,29

gekocht . . . . . . . . . . . 8,28

Rectum, Inhalt wie vor

ungekocht . . . . . . . . . 7,85

gekocht . . . . . . . . 7,03?

Die vorstehenden Werte sind auf Fig. 30 S. 344 graphisch dargestellt.

Fall Nr. 70.

Lutkat, Frieda, 21/2 Mon., Intoxikation bej grippaler Infektion mit Bronchopneumonie. Geb. 8. IV. 15, Geburtsgewicht ca. $4000 \mathrm{~g}, 4$ Wochen nur Brust, später Brust + Halbmilch bis 23. VI. 15. In den letzten 3 Tagen nur Halbmilch. Seit dieser Zeit Erbrechen und Durchfall, stark abgemagert. Am 26. V. aufgenommen, Gewicht $3300 \mathrm{~g}$, schwer benommen, Temp. 41,3, Schnupfen, Lungen anfangs o. B., dünne Stühle, kein Erbrechen. Bekommt mit Sonde kleine Mengen Frauenmilch und große Mengen bis $700 \mathrm{~g}$ Tee mit etwas Ringerlösung. Urin enthält viel Eiweiß und Zylinder, Zucker +, vom 27. bis zum Tode komplette Anurie, starke Gewichtszunahme bis $3900 \mathrm{~g}$, die ganze Zeit bewußtlos, am 29. Krämpfe, am 30 . Exitus unter Erscheinungen der Atemlähmung. Sektion gleich danach, Diagnose: Bronchopneumonie, trübe Schwellung der Nieren und hochigradige Leberverfettung. Über das klinische Verhalten siehe Näheres

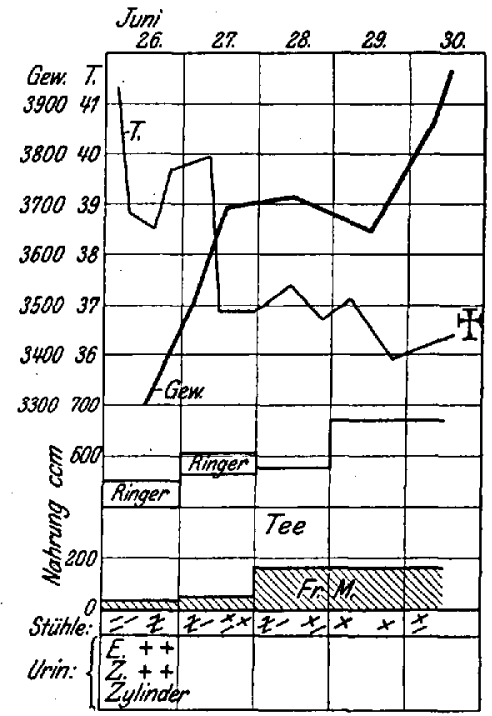

Fig. 84. Krankenkurve Fall 70 . Frieda Lutkat. in der beigefügten Gewichts. und Temperaturkurve Fig. 34. 
28. VI. ca. $5^{\text {h }}$ vorm.

Stuhl, spärlich, grünlich, schleimig . . . . . 5,88 derselbe Extrakt bei $28^{\circ}$ im Tonometer evakuiert 5,86

$5^{\text {h }}$ nachm. Stuhl, Beschaffenheit, wie vor . . . . . . 5,37

$6^{\text {h }}$ vorm. Urin, katheterisiert . . . . . . . . . . 5,61

$4^{\mathrm{h}}$ nachm. Urin, katheterisiert . . . . . . . . . . . . 5,37

$10^{\text {h }} 45^{\prime}$ Sinuspunktion, keine nennenswerte Reaktion dabei.

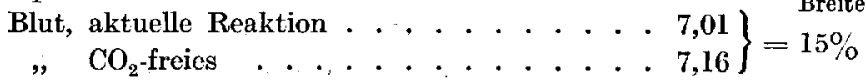

29. VI. $8^{\text {h }} 30^{\prime}$ vorm. nochmals Sinuspunktion, ohne Reaktion,

tief bewußtlos.

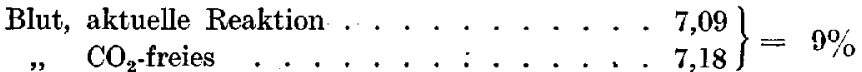

Stuhl, gelblich, salbig . . . . . . . . . 6,74

Urin, katheterisiert, trübe . . . . . . . . . 5,62

30. VI. $8^{\mathrm{h}} 30^{\prime}$ vorm. nochmalige Sinuspunktion, das Kind war

moribund und starb $10 \mathrm{Min}$. später.

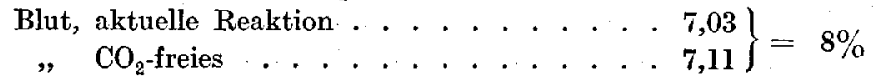

8h 40 Exitus.

Herzpunktion und Sektion gleich danach.

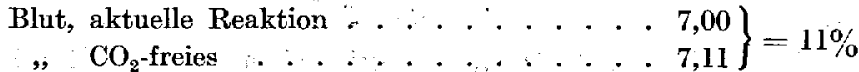

1. Muskulatur, beide Pectorales majores

ungekocht . . . . . . . . 6 6,60

gekocht . . . . . . . . . . 7,04

2. Muskulatur, beide Ileopsoas und Quadr. Iumb.

ungekocht . . . . . . . 6,52

gekocht . . . . . . . . . . 6,96

Herz, gespalten, mit Wasser gespült,

die eine Hälfte ungekocht . . . . . . 5,28

die andere Hälfte gekocht . . . . . . 6,28

Leber, ungekocht . . . . . . . . . . . 6,21

gekocht ........... . 6,50

Galle, dunkelgrün zäh, $4 \mathrm{ccm}$. . . . . 6,21

Milz, in der Mitte gespalten,

ungekocht . . . . . . . . . 6,39

gekocht . . . . . . . . . . . . 6,69

Niere; in der Mitte gespalten,

ungekocht . . . . . . . . 6,00

gekocht ............ 6, . . . . . .

Gehirn, mittels Punktion durch Orbita, trübe

Flüssigkeit, mit Gehirnmassen vermischt,

ungekocht . . . . . . . . 6,03

gekocht . . . . . . . . 6,38 
Inhalt des Magendarmkanals:

Magen enthält ca. $80 \mathrm{ccm}$ dicke, rahmige, homogene Flüssigkeit . . . . . . . . 4,46

Duodenum, Abschnitt von ca. $30-70 \mathrm{~cm}$ unterhalb Pylorus, leicht gelbliche zähe Flüssigkeit 5,06

Ileum, kurz oberhalb des Coecums, dickflüssige, gelbliche, homogene Flüssigkeit . . . 5, 5,

Coecum + Colon ascendens, gelbliche breige Masse . . . . . . . . . . . . 5,52

Rectum + Sigmoideum, gelblichgrüner dicker Brei . . . . . . . . . . . . . 5,87

Die Schleimhaut des Magen- und Darmkanals zeigte nur eine geringgradige Rötung, sonst o: B.

Sektionsdiagnose: siehe oben.

Die vorstehenden Werte für die Organacidität sind auf Fig. 28, II a. S. 335 und die für den Magendarminhalt auf Fig. 28, II b dargestellt. Das Verhalten des Blutes zeigt Fig. 22, II S. 325.

\section{Fall Nr. 71.}

Baartz, Werner, 8 Mon., Intoxikation, geb. 10. XII. 14, Geburtsgewicht $3250 \mathrm{~g}, 3$ Wochen Brust, später Milchmischungen, zuletzt Vollmilch 5-6 mal 12 Strich, Zwieback 1 mal. Bisher angeblich immer gesund gewesen und regelmäßig zugenommen. Am 26. VI. 15 plötzlich wässerige Stühle, bis zur Aufnahme am 30. VI. 10-15 täglich; Kein Erbrechen, kein Husten, seit 29. schlaff und müde, sehr gierig getrunken. Aufnahmegewicht $6370 \mathrm{~g}$, gut ernährtes Kind, stark wasserverarmt, deutlich benommen. Temp. $40,6^{\circ}$ bis $41^{\circ}$ bis kurz vor dem Tode. Stühle anfangs dünn und wässerig, am 2. VII. gar keinen. Urin: Eiweiß und Zucker +. Bekam in den zwei ersten Tagen nur Tee mit Sonde, am letzten Tage im ganzen $190 \mathrm{~g}$ Eiweißmilch mit $410 \mathrm{~g}$ Tee insgesamt. Tod unter Krämpfen am 3. VII. Sektionsbefund: Infektion der Magen- und Darmschleimhaut, Emphysema pulmonis, Hyperaemia cerebri.

I. VII. $7^{\text {h }} 40^{\prime}$ nachm. Blutentnahme durch Sinuspunktion,

Hirudin.

$" \mathrm{CO}_{2}$-freies ......... $\left.7,7,82\right\}=38 \%$

Lumbalpunktion:

Liquor klar, Eiweiß vermehrt . . . . . 7,75

$11^{\mathrm{h}}$ vorm. Urin, gelb, klar ........... . 5,79

$5^{\text {h }}$ nachm. Urin, gelb, klar . . . . . . . . . . . 5,69

2. VII. Sinuspunktion, leichte Reaktion dabei.

$\left.\begin{array}{c}\text { Blut, aktuelle Reaktion . . . . . . . . . } 7,48 \\ \text { 1. , } \mathrm{CO}_{2} \text {-freies (15 Min. im Tonometer) } . . .7,72\end{array}\right\}=24 \%$

2. $, \ldots, \quad(30:,, \quad, \quad, \quad) \ldots .7,73$

3. VII. $7^{\text {h }} 10^{\prime}$ vorm. Exitus.

$$
(30:,, \quad, \quad, \quad) . .7,73
$$

Herzpunktion und Sektion gleich danach.

$\left.\begin{array}{c}\text { Blut, aktuelle Reaktion . . . . . . . . . . . . . . . . } 6,61 \\ \text { " } \mathrm{CO}_{2} \text {-freies . . . . . . } 7,28\end{array}\right\}=67 \%$ 
Gehirnpunktion, leicht trübe Flüssigkeit . . . 6,79

Gehirnsubstanz, Stück von der Konvexitüt, ungekocht ........... 6,14

gekocht . . . . . . . . . . 6,22

Muskel, Pectoralis + Bauchmuskel, ungekocht . . . . . . . . . 7,21

gekocht : . . . . . . . . 7,28

Herz, gespalten, mit Wasser gespült,

ungekocht . . . . . . . . . 5,55

gekocht . . . . . . . 6, 6, . . .

Leber, stark verfettet,

ungekocht . . . . . . . 6,36

gekocht . . . . . . . . 6,45

Galle, zäh und dunkelgrün, $3 \mathrm{ccm}$. . . . 6,14

Milz, kaum vergrößert, ungekocht . . . . . . . . . . 6, 6,19

gekocht . . . . . . . . 6,18

Niere, gespalten, gespült,

ungekocht . . . . . . . . 6,10

gekocht . . . . . . . . . . 6,29

Inhalt des Magendarmkanals:

Magen, ea. $50 \mathrm{~cm}$ bräunliche, flockige Flüssig-

keit, mit vielem zähem Schleim vermischt . $\mathbf{3 , 9 9}$

Duodenum, Abschnitt von $60-100 \mathrm{~cm}$ unter-

halb Pylorus, gelbliche, schleimige, flockige

Flüssigkeit . . . . . . . . . . 6, 6,14

Ile u m, Abschnitt kurz vor Coecum, dunkel-

braune, dünne, flockige Flüssigkeit . . . . 6,24

Coecum und Colon ascendens, dunkelbraune,

salbige Massen . . . . . . . . . . . . 5,90

Rectu m, ein salbiger dunkelbrauner Knollen . 6,77

Die Sektion ergab nur Injektion der Magen- und Dïnndarmschleimhaut, Schwellung der Gehirnhäute, besonders auf der linken Hemisphäre, außerdem trübe Schwellung der Nieren und mäßige Verfettung der Leber. Die Tage vor der Erkrankung waren auffallend heiß, dies und der klinische Verlauf veranlaßten uns, den Fall als Hitzschlag-Intoxikation zu betrachten.

Die vorstehenden Werte sind auf Fig. 26 S. 333 graphisch dargestellt.

$$
\text { Fall Nr. } 72 .
$$

Schwitallik, Heinz, 3 Mon. 10 Tage, Intoxikation beim Brustkinde. Geb. am 8. IV. 1915, Geburtsgewicht $3500 \mathrm{~g}$. Bisher ausschließlich. Brustmilchernährung. Im Alter von 5 Wochen croupöse Pneumonie, die septisch pyämischen Verlauf nahm. Aufnahme im K.-A.-V.-H. am 12. V. Reahtsseitiges Empyem, Orchitis suppurativa und Pneumokokkenabscesse in den Bauchdecken, Thoracotomia. Danach langsame Besserung, wurde am 4. VII. im Alter von 3 Mon. geheilt entlassen. Dann 2 Wochen zu Hause munter und ganz gesund gewesen, am 18. VII. um $10^{\text {h }}$ nachm. noch gut an der Brust getrunken und ca. $11^{\text {h }}$ ein- 
geschlafen. Um $2^{\text {h }}$ nachts (19. VII.) erwachte die Mutter und morkte, daß das Kind ganz blaß war, es stöhnte stark, Schaum trat aus dem Munde, kleine Zuckungen im Gesicht und in den Extremitäten. Vorher immer guten Stuhl gehabt, nur ist der Mutter aufgefallen, daß der Junge in den letzten 3 Tagen eine etwas heisere Stimma hatte. Um $3^{\text {h }} 30^{\prime}$ nachts brachte die Mutter das Kind wieder in das K.-A.-V.-Haus. Es war schwer kollabiert, tief benommen, stark spastisch, zeitweise klonische Zuckungen, keine Reaktion beim stärksten Kneifen. Über den Unterlappen der rechten Lunge spärliches kleinblasiges feuchtes Rasseln, deutliche Rötung des Rachens, sonst Herz und Lunge o. B. Hatte bei der Aufnahme in der Windel reichliche Mengen von gutem, gelblich-salbigem Stuhl. Temp. $39,8^{\circ}$. Der nächste Stuhl aber, der um $5^{\text {h }}$ entleert wurde, war schleimig und eiterig und mit Blutstreifen vermischt. Exitus um $6^{\mathrm{h}} 30^{\prime}$.

Sektion ca. 3 Stunden später.

Herzpunktion zur gleichen Zeit.

Blut, aktuelle Reaktion . . . . . . . 6,59

, $\mathrm{CO}_{2}$-freies . . . . . . . . 6,97

Muskulatur beiderseits Pectoralis majoris, ungekocht . . . . . . . . 6,10

gekocht . . . . . . . . . . 6,19

Herz, gespalten, mit Wasser gespült, ungekocht . . . . . . . . . 5,75

gekocht . . . . . . . 6, 6, . . . . . . .

Leber, ungekocht . . . . . . . . 6,50

gekocht . . . . . . . . . 6,55

Milz, stark vergrößert, pulpös, blutreich,

ungekocht . . . . . . . . 6,73

gekocht . . . . . . . . 6,61

Niere, ungekocht . . . . . . . 6, 6,47

gekocht . . . . . . . 6,54

Gehirnsubstanz von der Konvexität

ungekocht . . . . . . . . . 7,09

gekocht . . . . . . . . . . 7,19

Die vorstehenden Werte sind auf Fig. 28, I S. 335 graphisch dargestellt.

Die Sektion ergab neben älteren Verwachsungen der linken Lunge nur Gehirnödem und Injektion der Darmschleimhaut.

\section{Fall Nr. 73.}

Totgeburt, Kind N., ausgetragen, $4000 \mathrm{~g}$, Schwangerschaft ca. 9 Mon. lang normal, dann Wehen, in deren Verlauf am 30 . VII $10^{\text {h }}$ vorm. die Herztöne des Foetus aufhörten. Geburt um $4^{\text {h }}$ nachm. Das Kind tot, Nabelschnur um den Hals.

Sektion gleich danach.

Blutentnahme durch Herzpunktion.

Blut, aktuelle Reaktion . . . . . . 6, 6,17

, $\mathrm{CO}_{2}$-freies ........ 6,68 


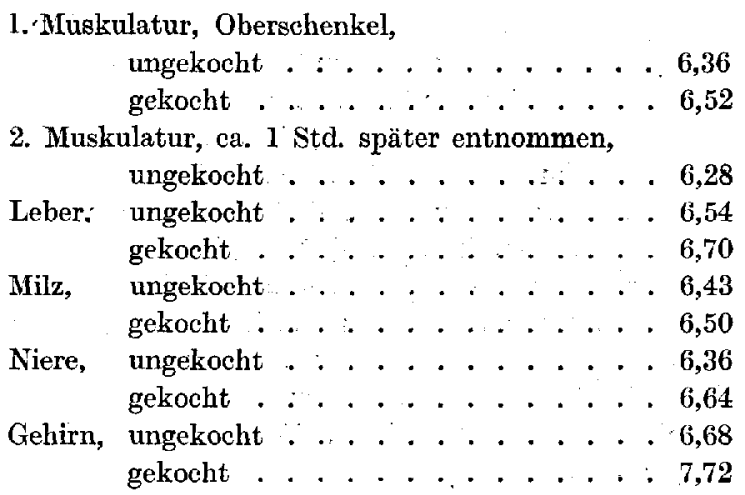

Die Sektion ergab eine starke Blutüberfüllung verschiedener Organe, sonst nichts Besonderes.

Die vorstehenden Werte sind auf Fig. 12 graphisch dargestellt.

Fall Nr. 74.

Wieland, Georg, 31/2. Mon. Intoxikationszustand leicht und vorübergehend während chronischer schwerer Ernährungsstörung. Geb. am 16. III. 15. Geburtsgewicht ünbekannt, ausgetragenes Kind. 14 Tage Brust, später bis zur Erkrankung $1 / 2$ Milch, dabei gute Zunahme bis zum 20. VI.; dann plötzlich dünne Stühle, die bis zur .Aufnahme im K.-A.-V.-H. am 3. VII. unverändert geblieben sind, trotzdem das Kind 2 Wochen lang nur Haferschleim mit etwas Zucker bekommen hat. Stark abgenommen, kein Erbrechen. In der Familie Polymortalität, von 7 Kindern starben 4 in den ersten. Monaten an Ernährungsstörung.

3. VII. Status: bei der Sinuspunktion:

Blasses, stark abgemagertes Kind von $3500 \mathrm{~g}$ mit eingesunkenem Leib, macht sehr müden, leicht apathischen Eindruck, fixiert aber und reagiert deutlich bei der Sinuspunktion. Stïhle spärlich, dunkelbraun (Hunger-

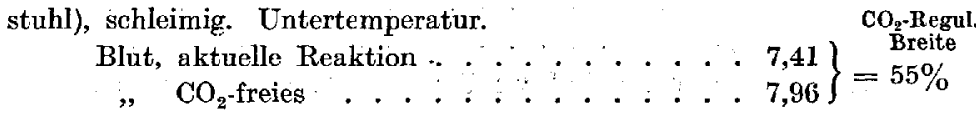

Am 5. VII. deutlich benommen, trinkt schlecht, bekommt Phosphatgemisch und Eiweißmilch mit 3\% Soxhletzucker 6 mal $40 \mathrm{~g}$. Am 7. VII. fixiert es wieder etwas, doch noch sehr apathisch, müde und schlaff.

7: VII. Sinuspunktion $1^{\mathrm{h}}$ mittags:

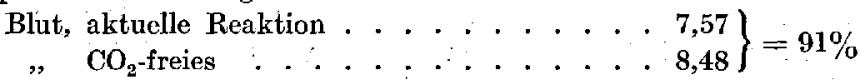

9. VII. trinkt etwas besser, noch müde, seit vorgestern keinen

Stuhl. Sinuspunktion $1^{\text {h }}$ nachm.

Blut, aktuelle Reaktion. . . . . . . . . . . 7,37

$\left.\begin{array}{c}\text { " } \mathrm{CO}_{2} \text {-freies (25 Min. im Tonometer) } 1.8,54 \\ \quad " \quad(35,,, \quad, \quad 2.8,45\end{array}\right\}$ Mittel 8,50 $\}=113 \%$ 
20. VII. hat sich ausgezeichnet erholt, Gewicht $3780 \mathrm{~g}$, täglich

$720 \mathrm{~g}$ Eiweißmilch mit $3 \%$ Soxhletzucker. Lebhafter, trinkt gut.

Sinuspunktion:

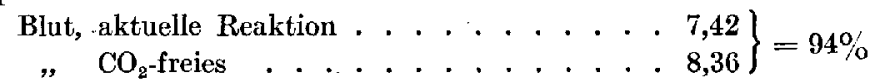

26. VII. $3800 \mathrm{~g}$, blaB, lacht aber schon. Tee + , Eiweißmileh.

Sinuspunktion $1^{\mathrm{h}}$ nachm.:

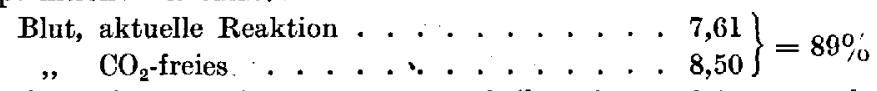

Am 30. IX. im Alter von 6 Mon. mit $4540 \mathrm{~g}$ geheilt entlassen. Die vorerwähnten Zahlen für die Blutreaktion sind auf Fig. 22, III S. 325 graphisch dargestellt.

Fall Nr. 75.

S., Hermann, 4 Mon. Intoxikation im Verlaufe von akuter Ernährungsstörung. Geb. 17. III. 15, Geburtsgewicht 3500 g. Nur 8 Tage Brust, später bis zur Aufnahme 5 mal 150-200 g Vollmilch mit nur wenig Wasser verdünnt. Am 10. VII. dünne Stühle und Erbrechen, 2 Tage lang Haferschleim, dann geringe Mengen Eiweißmilch, dann Nestlesches Kindermehl, zuletzt Eiweißmilch $220 \mathrm{~g}$ pro Tag. Stühle zahlreich, bis 8 täglich, müde, schlaff geworden.'Am 20. VII. im K.-A.:V.-H. aufgenommen.

Status: Gut entwickeltes Kind mit schlaffem reduziertem Fettgewebe, macht müden, wasserverarmten Eindruck, etwas Starres im Wesen, folgt wohl auf starke Reize: Licht und Klatschen, reagiert aber wenig z. B. bei der Untersuchung des Rachens. Lungen und Herz o. B., Stühle spritzend und dünn. Bekommt in den ersten Tagen neben Tee geringe Mengen Eiweißmilch, später neben Phosphatgemisch nur Frauenmilch. Genaueres über die Nahrungs- und Fieberverhältnisse (kontinuierliches Fieber!) siehe in der Kurve Fig. 29. Im Urin Eiweiß +, Zucker +, im Sediment Cylinder.

22. VII. deutlich apathisch, liegt ruhig mit weit geöffneten Augen im Bett, hat aber leidlich gut getrunken. Leichte Reaktion bei-der

Sinuspunktion um $12^{\mathrm{h}}$ mittags

$$
\begin{aligned}
& \text { Blut, aktuelle Reaktion Breite } \\
& \text { " } \left.\mathrm{CO}_{2} \text {-freies . . . . . . . . . . . . . . . } 7,90\right\}=46 \%
\end{aligned}
$$

23. VII. leicht benommen, am Nachmittag Krämpfe, seitdem deutlich toxisch. Seit $8^{\mathrm{h}}$ vorm. Phosphatgemisch, dessen $P_{H}=7,41$, neben Frauenmilch mittels Sonde. Siehe genauer die Kurve

Urin ............. . $5,6 \mathrm{I}$

Stuhl, dünn, breiig, schaumig . . . . . 6,77

24. VII. dem Aussehen nach deutlich toxisch, hat aber heute selbst etwas getrunken und reagierte mit deutlichem Schreien bei der Sinuspunktion $10^{\text {h }} 50^{\prime}$ vorm. $10^{\mathrm{h}} 50^{\prime}$ vorm.

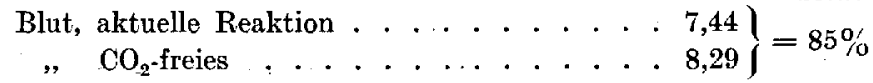


Stuhl, grau, salbig (Eiweißmilchstuhl) . . . 6,74

Urin, Zucker — . . . . . . . . . 6,46

$2^{\mathrm{h}}$ nachm. Urin . . . . . . . . . . . . . . . . 7,15

26. VII. noch ganz leicht benommen, liegt mit halboffenen

Augen ruhig im Bett, beim Anfassen Zeichen von Un. willeri.

Urin . . . . . . . . . . . . . . . 7,05

Stuhl, gelbliche, bröcklige, salbige Masse mit einzelnen grünlich schimmernden Schleimflocken 5,54

27. VII. noch miide, leicht apathisch, reagiert aber mit Schreien bei der Sinuspunktion um $10^{\text {h }} 50^{\prime}$ vorm.

Blut, aktuelle Reaktion . . . . . . . 7,36

, $\mathrm{CO}_{2}$-freies ........... 8,26$\}=90 \%$

$1^{\text {h }}$ mitt. Stuhl gelb, porös, leicht salbig, Spur grünlicher

Schleim . . . . . . . . . 5,67

Urin . . . . . . . . . . 7,30

28. VII. $1^{\text {h }}$ vorm.

Urin . . . . . . . . . . . . . . 6,99

$1^{\text {h }}$ mitt. Stuhl, gelbliche, flockige Salbe . . . . . 5,55

Die Werte für die Blutreaktion sind auf Fig. 22, I S. 325 graphisch dargestellt. Fall Nr. 76.

Suckrow, Alfred, 19 Tage alt, Intoxikation beim Brustkinde. Geb. 24. VIII. 14, ausgetragen, bisher nach vollkommen zuverlässigen Angaben nur Brust bekommen. Am 9. IX. plötzlich dünne Stühle und heiß, trank aber noch gut an der Brust, am 10. IX. schlecht getrunken, vom 11. bis 13. die Brust verweigert, nur etwas Tee geschluckt, am 12. auffallend apathisch geworden, Stühle noch dünn, kein Erbrechen, am 13. zur Aufnahme gebracht.

13. IX. Status: Leidlich ernährtes tief toxisches, schwer kollabiertes Kind, Gewicht $3300 \mathrm{~g}$. Lungen und Herz o. B. Bekommt im Verlaufe des Tages mit Sonde im ganzen $50 \mathrm{~g}$ Frauenmilch und $150 \mathrm{~g}$ Tee, außerdem subcutan $120 \mathrm{~g}$ Ringerlösung. Temp. $38^{\circ}$, Stühle 8 mal dünn, gelblich. Trotz Camphers und Senfbades am 14. IX. um $8^{\text {h }}$ früh, 20 Stunden nach Aufnahme, Exitus. I4. IX. $8^{\mathrm{h}}$ vorm. Herzpunktion und Sektion gleich danach.

Blut, aktuelle Reaktion . . . . . . . . 6,85

" $\mathrm{CO}_{2}$-freies . . . . . . . . . . . 6,92

(Die Dissoziationskurve siche später.)

Blutkörperchen, 3 mal mit $\mathrm{NaCl} 0,85 \%$ gewaschen, mit destill. $\mathrm{H}_{2} \mathrm{O}$ hämolysiert . . . . . . . 6,61

Gehirnpunktat leicht rosa verfärbt, mit Gehirnmassen vermischt. . . . . . . . . 6,41

Leber, ungekocht . . . . . . . . . 6,31 gekocht . . . . . . . . 6,53

Galle ca. $5 \mathrm{ccm}$, grünlich, zäh . . . . . . 6,87

Muskel, Pectoralis ungekocht . . . . . . . . . 6,71

gekocht . . . . . . . . 6,88 
und Intoxikationsacidosis in ihren Beziehungen zueinander.

Niere, ungekocht . . . . . . . 5,86

gekocht . . . . . . . . 5,93

Inhalt des Magen- und Darmkanals:

Magen, ca. $10 \mathrm{ccm}$ dunkelbraune, zähe, schleimige

Flüssigkeit . . . . . . . . . 6,83

Duodenum, Abschnitt von $60-100 \mathrm{~cm}$ gelblich-

braune, flockige Flüssigkeit . . . . . 7,57

Ileum, Abschnitt kurz vor Coecum, schokoladen-

braune, flockige Flüssigkeit . . . . . 7,38

Rectum, weißlichgelbe, dünnflüssige Brühe . . 6,93

Die Sektion ergab starke hämorrhagische Verfärbung der Magen- und Dünn. darmschleimhaut mit mehreren Blutaustritten. Colon und Rectumschleimhaut zeigten nur geringe Injektion. Diagnose: Gastritis und Enteritis acuta haemor. rhagica.

Die vorstehenden Werte sind auf Fig. 27 graphisch dargestellt. Weiterhin wurde noch die $\mathrm{O}_{2}$-Dissoziationskurve des Blutes bestimmt:

\section{Punkt.}

3,0 ccm Herzblut im Tonometer, daselbst ununterbrochen 25 Min. geschüttelt bei $38^{\circ}$.

$\left.\begin{array}{lll}\text { Temp. : } 19^{\circ} & \text { Gasanalysen } & \mathrm{I}=2,6 \\ \text { Druck: } 750 \mathrm{~mm} \mathrm{Hg} & \text { aus dem Tonometer } & \text { II }=2,6\end{array}\right\}=2,6 \% \mathrm{O}_{2}$ Differentialmanometer I bei $17^{\circ} \mathrm{im}$ Wasserbade.

Gleichgewicht bei . . . . . In die linke Birne In die rechte Birne das gesättigt e Blut das zu untersuch. Blut Differenz $\begin{array}{llll}\text { Nach dem Schütteln.... } & 6,64 & \mathbf{8 , 6 0} & \mathbf{2 , 0 1}\end{array}$

Kaliumferricyanid rechts,

Hähne vorher nicht geöffnet

8,22

7,05

1,13

$$
\begin{array}{ll}
\% \text { Sättigung } & =36,0 \% \mathrm{O}_{2} \\
\mathrm{O}_{2}-\mathrm{Druck} & =19,5 \mathrm{~mm} \mathrm{Hg} \\
\mathrm{P}_{\mathrm{H}} & =6,92
\end{array}
$$

II. Punkt.

Tonometer wie vor.

Temp. : $19^{\circ}$

Druck: $750 \mathrm{~mm} \mathrm{Hg}$

$\left.\begin{array}{lr}\text { Gasanalysen } & \mathrm{I}=5,2 \\ \text { aus dem Tonometer } & \mathrm{II}=5,4\end{array}\right\}=5,3 \% \mathrm{O}_{2}$

Differentialmanometer II bei $17^{\circ} \mathrm{im}$ Wasserbade.

Gleichgewicht bei . . . . .

In die linke Birne In die rechte Birne

das gesăttigte Blut das zu untersuch. Blut Differenz

$6,70 \quad 8,04$

1,34

Kaliumferricyanid rechts,

Hähne vorher nicht geöffnet

$$
8,47
$$

6,24

$$
\begin{aligned}
& \% \text { Sattigung }=62,5 \% \mathrm{O}_{2} \\
& \mathrm{O}_{2}-\mathrm{Druck}=39,75 \mathrm{~mm} \mathrm{Hg} \\
& \mathrm{P}_{\mathrm{H}} \text { wie vor. }
\end{aligned}
$$

Aus der Gleichgewichtsformel wurden die Werte für $n$ und $K$ berechnet:

$$
\begin{aligned}
\mathbf{n} & =\mathbf{2 , 0} \\
\mathbf{K} & =0,0014 ;
\end{aligned}
$$


mit Hilfe dieser Zahlen wurde dann Punkt III bestimmt:

$$
\begin{aligned}
& \% \text { Sättigung }=85,0 \% \\
& \mathrm{O}_{2} \text {-Druck }=63,8 \mathrm{~mm} \mathrm{Hg} .
\end{aligned}
$$

Die Dissoziationskurve ist auf Fig. 24, III S. 330 graphisch gezeichnet.

$$
\text { Fall Nr. } 77 .
$$

Cornet, Frieda, 3 Mon., Intoxikation im Verlaufe von grippaler Erkrankung bei chronisch ernährungsgestörtem Kinde. Geb. 10. IX. 14. Geburtsgewicht 3450 g. 4 Wochen ausschließlich Brust, später Zwiemilchernährung, im letzten Monat nur $1 / 2$ Milch. Dabei gut zugenommen, wog am 21. XI. schon $4000 \mathrm{~g}$, hatte aber immer röchelndes Atmen; in der Fürsorgestelle Bronchitis und Nasopharyngitis chronica konstatiert. Anfang Dez. wieder starken Schnupfen und Husten, dünne Stïhle; starke Abnahme, wird am 7. XII. deswegen zur Aufnahme gebracht.

7. XII. Status: Stark atrophisches Kind, $3140 \mathrm{~g}$, deutlich benommen, Stühle dünn, und heftiges Erbrechen. Bekommt täglich 120-200 g Ringerlösung subcutan, außerdem mit Sonde langsam steigend $100-400 \mathrm{~g}$. Tee und 50-350 g Frauenmilch, die letzten Mengen am Tage vor dem Exitus. Temperatur von $37^{\circ}$ bis $40^{\circ}$ schwankend. Am 8. XII. kaum mehr toxisch, nur sehr schlaff, fixiert etwas, Urin trübe, Eiweiß +, Zucker +, viel Leukocyten.

Blut, aktuelle Reaktion . . . . . . . . . 7,64

Lumbalflüssigkeit, klar . . . . . . . 7,98

14. XII. wieder toxisch, hustet, Verdacht auf Bronchopneunonie.

Exitus heute $7^{\text {h }}$ früh.

Sektion und Herzpunktion ca. 4 Stunden später.

Blut, aktuelle Reaktion . . . . . . . . . . . 6,87

" $\mathrm{CO}_{2}$-frejes ......... . . 7,86

(Die Dissoziationskurve des Hämoglobins siehe

unten.)

Gehirnflüssigkeit, rosa, trübe . . . . . 6 6,42

Muskel, Pectoralis

ungekocht . . . . . . . . . 6,78

gekocht . . . . . . 6, 6,78

Leber, ungekocht . . . . . . . . . 6,15

gekocht . . . . . . 6,24

Galle, zäh, grün . . … . . . . 6,04

Milz, ungekocht . . . . . . . 6,15

gekocht . . . . . . . . 6, 6,27

Dissoziationskurve des Hämoglobins.

Bestimmung I.

2,0 ccm Herzblut im Tonometer.

Im Tonometer 25 Min. geschüttelt, nach dem Schütteln: klar.

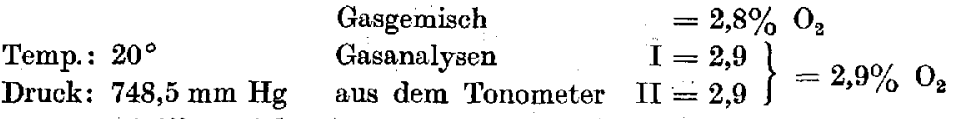

Differentialmanometer im Wasserbade bei $38^{\circ}$.

In die Birne ea. 0,1 com Blut. 


\begin{tabular}{|c|c|c|c|}
\hline Gleichgewicht bei & $\begin{array}{c}\text { In die linke Birne die } \\
\text { gesättigte Blutlös. } \\
7,39\end{array}$ & $\begin{array}{c}\text { In die rechte Birne die } \\
\text { zu untersuch. Blutlös. } \\
7,34\end{array}$ & Differe \\
\hline Nach dem Schütteln & 8,69 & 9,99 & 1,35 \\
\hline \multicolumn{4}{|l|}{ Kaliumferricyanid rechts, } \\
\hline Hähne vorher nicht geöffnet & 10,12 & 8,54 & 1,53 \\
\hline \multicolumn{4}{|c|}{$\begin{array}{l}\% \text { Sättigung }=53,1 \% \mathrm{O}_{2} \\
\mathrm{O}_{2} \text {-Druck }=21,7 \mathrm{~mm} \mathrm{Hg} \\
\text { E.M.K. nach dem Schütteln= } 703,0 \text { Millivolt bei } 17^{\circ} \\
\mathrm{P}_{\mathrm{H}} \quad=7,86\end{array}$} \\
\hline
\end{tabular}

A. Manometer I bei $38^{\circ}$, in die Birne $0,1 \mathrm{ccm}$ Blut.

Temp.: $20^{\circ}$; Druck: 748,5 $\mathrm{mm} \mathrm{Hg}$.

In die linke Birne die In die rechte Birne die gesulttigte Blutlös. zu untersuch. Blutiős. Differenz

Gleichgewicht bei . . . . . $\quad 9,25$

$\begin{array}{lll}9,25 & 9,25 & \\ 8,96 & 9,96 & 1,00\end{array}$

$\begin{array}{llll}\text { Nach dem Schütteln . . . . } & 8,96 & \mathbf{9 , 9 6} & \mathbf{1 , 0 0}\end{array}$

Kaliumferrioyanid rechts,

$\begin{array}{llll}\text { Hähne vorher nicht geöffnet } & 10,38 & 8,30 & 2.08\end{array}$

B. Manometer II bei $38^{\circ}$, in die Birne etwas weniger als $0,1 \mathrm{ccm}$ Blut.

Gleichgewicht bei . . . . $\quad 7,39 \quad 7,30$

$\begin{array}{llll}\text { Nach dem Schütteln . : }: ~ & 7,89 & \mathbf{7 , 7 0} & 0,90\end{array}$

Kaliumferricyanid rechts,

$\begin{array}{llll}\text { Hähne vorher nicht geöffnet } & 9,24 & 7,40 & 1,75\end{array}$

$$
\begin{aligned}
& \begin{array}{l}
\left.\% \text { Sättigung } \begin{array}{l}
\mathrm{A}=67,5 \\
\mathrm{~B}=66,0
\end{array}\right\}=66,8 \% \mathrm{O}_{2} \\
\mathrm{O}_{2} \text {-Druck } \\
\mathrm{P}_{\mathrm{H}}=\text { Bestimmung } \mathrm{I} .
\end{array}=31,43 \mathrm{~mm} \mathrm{Hg}
\end{aligned}
$$

Die Sektion ergab: Bronchitis chronica; Bronchopneumonie, Nephritis parenchymatosa, geringfügige Injektion der Darmschleimhaut.

Die vorstehenden Zahlen außer den für die Dissoziationskurve sind anf Fig. 31, I S. 345 graphisch dargestellt.

\section{Fall Nr. 78.}

Kreutzer, Erwin, 6 Mon. alt, Intoxikation im Anschluß an akute Ernährungsstörung, Bronchopneumonie. Geb. 20. II. 14, ausgetragen, von Anfang an bis zur Erkrankung nur $1 / 3$ Milch mit 1 Teelöffel Rohrzucker 8 mal täglich $3-4^{\prime}$. War sehr mager, Stühle waren gut, und soll das Kind sonst bis zum 13 . VIII. gesund gewesen sein. Dann heftiges Erbrechen und dünne, schleimige Stühle, sehr unruhig, vom 16. angefangen nur Haferschleim. In der Nacht zum 17. VIII. schläfrig, heute früh gar nichts mehr getrunken, deswegen zur Aufnahme gebracht. 17. VIII. Status: Stark wasserverarmtes, atrophisches Kind, tief toxisch, Temp. $40^{\circ}$, Stühle blutig-eiterig, dünn. Dämpfung über dem rechten Oberlappen. Bekam im Verlaufe des Tages mit Sonde $280 \mathrm{~g}$ Tee und $20 \mathrm{~g}$ Frauenmilch, die teilweise wieder erbrochen wurden. Außerdem subeutan $2 \mathrm{mal} 150 \mathrm{~g}$ Phosphatgemisch, das langsam resorbiert wurde.

Stuhl, schleimig, blutig . . . . . . . 6,68

Urin, trübe (Eiweiß +, Zucker +) . . 5, 5,37

18. VIII. Exitus $7^{\text {h }}$ nachm.

Herzpunktion und Sektion gleich danach. 


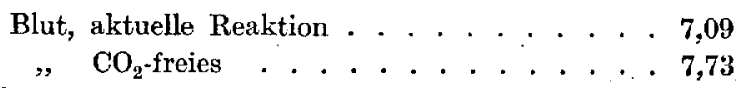

Ein Punkt der Dissoziationskurve des Hämoglobins wurde wie folgt bestimmt 2,0 cem Herzblut im Tonometer, daselbst 25 Min. geschüttelt.

Temp.: $19^{\circ}$

Druck: $753 \mathrm{~mm} \mathrm{Hg}$ aus dem Tonometer $\left.\begin{array}{r}\mathrm{I}=3,5 \\ \mathrm{II}=3,4\end{array}\right\}=3,5 \% \mathrm{O}_{2}$

Differentialmanometer im Wasserbade bei $18^{\circ}$.

In die Birne ca. 0,1 ccm Blut.

Gleichgewicht bei . . . . .

In die linke Birne die In die rechte Birne die

$\begin{array}{llll}\text { Nach dem Schütteln . . . . } & 6,95 & \mathbf{7 , 9 3} & 0,98\end{array}$

Kaliumferricyanid rechts,

Hähne vorher geöffnet . $\quad 8,60 \quad 6,25 \quad 2,35$

$$
\begin{aligned}
& \% \text { Sätigung }=58,3 \% \mathrm{O}_{2} \\
& \mathrm{O}_{2} \text {-Druck }=26,36 \mathrm{~mm} \mathrm{Hg} \\
& \mathrm{CO}_{2} \text {-freies Blut aus dem Tonometer } \mathrm{P}_{\mathrm{H}}=7,73
\end{aligned}
$$

Acidität der Organe in frischem wässerigem Extrakt:

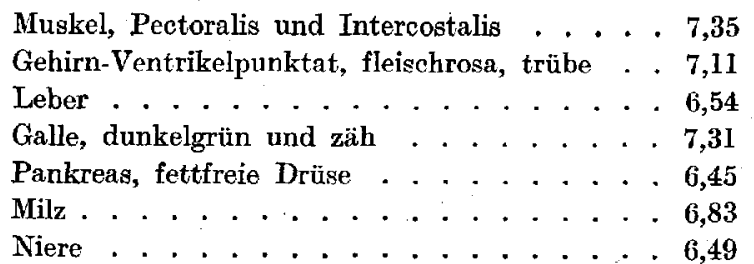

Aciditãt des Magen- und Darminhalts:

Magen, schleimig, flockige Flüssigkeit . . . . 4,41

Duodenum, Abschnitt $60-75 \mathrm{~cm}$ unterhalb Py.

lorus, gelblich bräunliche, flockige Flüssigkeit 6,44

Dünndarm, Abschnitt kurz vor Coecum, braune

schleimige dünne Flüssigkeit . . . . . . 6,94

Rectum, gelblichbrauner, dünnflüssiger Brei . . 7,13

Die Sektion ergab: Bronchopneumonie und starke Injektion der Magenund Darmschleimhaut.

Die vorstehenden Werte sind auf Fig. 31, II S. 345 graphisch dargestellt.

$$
\text { Fall Nr. } 79 .
$$

Hueckstedt, Frieda, .6 Wochen alt. Intoxikation im AnschluB an akute Ernährungsstörung. Ausgetragen, am 30. VII. 14 geb. 4 Wochen Brust, dabei leidliche Zunahme, dann 3' Milch und 3' Wasser 6 mal täglich. Bekam aber bald (am 8. IX.) dünne Stühle und hat seit dieser Zeit 6 Tage lang nur Tee und Reisschleim getrunken. Stühle trotzdem dünn, auch ab und zu gebrochen. Wurde am 13. IX. schläfrig, trank nichts mehr, und deswegen am 14. IX. zur Aufnahme gebracht. Status: mageres Kind, deutlich benommen, mehrere Furunkel am Kopfe, Urin: Eiweiß +, Zucker -, im Sediment viel Leukocyten und einzelne Cylinder. Bekommt geringe Mengen Frauenmilch, von 50 bis $300 \mathrm{~g}$, etwas Tee und außerdem an den zwei ersten Tagen subcutan $100 \mathrm{~g}$ Ringerlösung. Temp. 
$37^{\circ}$ bis $39^{\circ}$. Blicb ununterbrochen toxisch, nabm langsam ab, von 3100 bis $2900 \mathrm{~g}$, es entstanden neue Furunkel, Urin unverändert, nach langsamer Agonie Exitus am 22. IX. $7^{\text {h }} 50^{\prime}$ vorm. Herzpunktion und Sektion gleich danach.

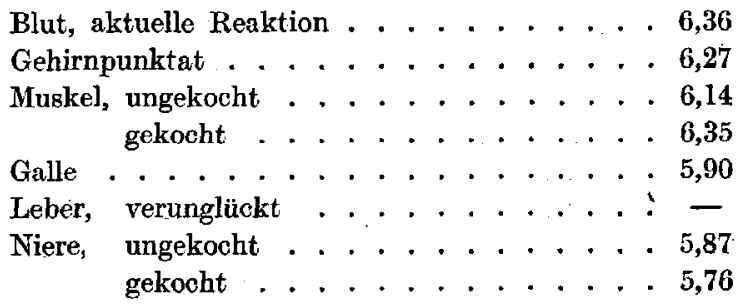

Inhalt des Magen- und Darmkanals:

Magen, weißflockige; schleimige Flüssigkeit . . 5,92

Duodenum, Abschnitt $60-90 \mathrm{~cm}$ unterhalb Py-

lorus, gelbe flockige Brühe . . . . . 5,70

Coecum, gelbliche, dickflüssige Brühe . . . 6,43

Rectum, gelbliche schleimige Salbe . . . . . 7,10

Sektion ergab: Schwellung und Rötung der Dickdarmschleimhaut, Furunkulosis, Atrophia universalis.

\section{Fall Nr. 80.}

Dokter, Herbert, $1^{1 / 2}$ Mon. alt, schwere chronische Ernährungsstörung. Bekam von Geburt an 1/2 Milch mit etwas Zucker, dabei Stühle immer dünn, ständige Abnahme, Geburtsgewicht $4000 \mathrm{~g}$, Aufnahmegewicht am 21. IX. I4 3350 g. Status: mageres, schlaffes Kind, sieht schwach, aber nicht toxisch aus. Bekommt zuerst $1 / 3$ Milch (Larosan), die Stühle bleiben aber dünn, dabei weitere Abnahme, deswegen vom 26. an $\mathbf{4 0 0} \mathrm{g}$ täglich gereicht. Trotzdem Fieber, weitere Abnahme und ständiger Verfall und ohne besonderen vorhergehenden Intoxikationszustand am 29. IX. tot. Gewicht dann $2900 \mathrm{~g}$. 29. IX. $10^{\text {h }}$ nachm. Herzpunktion und Sektion gleich nach dem Exitus.

Blut, aktuelle Reaktion . . . . . . . 6,77

Gehirnsubstanz und -flüssigkeit . . . . . 6,79

Fall Nr. 81.

Wilbelmi, Leonor, 3 Mon. Intoxikation im Verlaufe von chronischer schwèrer Ernährungsstörung (Mehlnährschaden). Geb. 20. VI: 14 mit $3250 \mathrm{~g}$, verkrüppelte Beine, wird von Anfang an mit $1 / 3$ Milch und Rademanns Kindermehl ernährt, gedeiht schlecht, hat andauernd Durchfälle. Am 28. IX. deswegen aufgenommen. Status: mageres, elend aussehendes, verkrüppeltes Kind, Gewicht $3130 \mathrm{~g}$, tief benommen, Untertemperatur, bekommt am ersten Tage Tee, ca. $200 \mathrm{~g}$ mit Sonde, außerdem 100. $\mathrm{g}$ Ringerlösung, am folgenden Tage außerdem noch $50 \mathrm{~g}$ Frauenmilch, hat in den 2 Tagen nur 3 mal ganz spärlichen schleimigen Stubl gehabt. Unter zunehmendem Verfall und nach tiefem Koma Exitus am 30.IX. 30. IX. $6^{\text {h }}$ früh Herzpunktion und Gehirnpunktion gleich nach dem Exitus.

Blut, aktuelle Reaktion . . . . . . . . . . 6,87

Gehirnpunktat, fleischsaftartige Flüssigkeit mit

Gehirnmassen vermischt . . . . . . 6,72 
Fall Nr. 82.

Weger, Gustav; 5 Wochen alt, Intoxikation beim Brustkinde. Geb. 31. VII. 14, ausgetragenes Kind, Geburtsgewicht unbekannt. Bis zur Aufnahme nur Brust bekommen, regelmäßig und gut zugenommen, wog am 3. IX. schon $3900 \mathrm{~g}$. An demselben Tage wurde das bisher ganz gesunde Kind unruhig, faßte die Brust schlecht an, die Stühle wurden dünn, waren am 4. IX. wässerig, 10 bis $15 \mathrm{im}$ Verlaufe des Tages. Am 5. IX. sehr schläfrig, trank nicht mehr an der Brust, schluckte nur etwas Tee. Die Schläfrigkeit und die dünnen Stühle dauerten bis zum 7. IX. an, an welchem Tage das Kind zur Aufnahme gebracht wurde. Status: Stark abgemagertes, wasserverarmtes Kind, benommen, Atemfrequenz nur 14 pro Min., Lunge und Rachen o. B. Stühle dünn, schleimig, nur 2 täglich. Gewicht $3000 \mathrm{~g}$, also im ganzen ca. $900 \mathrm{~g}$ in 4 Tagen abgenommen. Das Kind bekommt gleich Ringerinfusion $150 \mathrm{~g}$, außerdem am ersten Tage nur Tee, an den folgenden Tagen neben Tee Frauenmilch von $150 \mathrm{~g}$ bis $500 \mathrm{~g}$ täglich steigend. Das Sensorium wird allmählich klar, am 11. fixiert das Kind bereits und guckt lebhaft um sich. Die Stüble waren schon am 8. IX. gut geformt, am 9. und 10. gar kein Stuhl, später war derselbe immer gut. Kein Erbreehen während der ganzen Erkrankung. Am 7. IX. Untertemperatur, vom 8. bis 11. IX. von $37,3^{\circ}$ bis $37,6^{\circ}$, dann monothermisch $36,8^{\circ}$. Später bekam das Kind in der Klinik leichte Bronchitis mit Fiebersteigerungen, entwickelte sich aber trotzdem gut und wurde am 14. XII. mit $1 / 2$ Milch-Ernährung bei $4600 \mathrm{~g}$ Gewicht entlassen. 8. IX. $8^{\text {h }}$ vorm. Sinuspunktion, deutlich benommen, doch leichte Reaktion bei der Punktion.

Blut, aktuelle Reaktion . . . . . . . . 7,09

Bemerkung: Deutliche Steigerung der Acidität des Blutes bei einer nicht tödlich verlaufenden Intoxikation.

\title{
VIII. $\mathrm{O}_{2}$-Dissoziationskurve und die $\mathrm{CO}_{2}$-Regulationsbreite des Blutes bei Intoxikation.
}

\author{
Fall Nr. 83.
}

Müller, Werner, 21/4 Mon., Intoxikation im Anschluß an akute Ernährungsstörung, am 12. VI. 14 geb. mit $4000 \mathrm{~g}$ Gewicht. 8 Tage Brust, später $1 / 3-1 / 2$ Milch, gedieh damit leidlich bis zum 9. VIII. Dann dünne Stühle und Abnahme. Vom 14. VIII. Eiweißmilch mit ca. 3\% Soxhletzucker 6 mal 3r. Damit wurden die Stühle zunächst gelb, am 17. wieder etwas dünner, das Kind fing an heftig zu erbrechen, verfiel stark, wurde allmählich ganz apathisch und so am 18. VIII. zur Aufnahme gebracht. Status: Sohwer toxisches Kind in leidlichem Ernährungszustande, stark wasserverarmt. Gewicht 3800 g. Starker Soor. Stühle zerfahren, schleimig; Urin: Eiweiß +, Zucker -, im Sediment granulierte Cylinder. Bekommt täglich 100-125 ccm Ringerlösung, außerdem am ersten Tage Tee, im ganzen $120 \mathrm{ccm}$, am folgenden Tage etwa die gleiche Menge und außerdem von 50 - $180 \mathrm{~g}$ Frauenmiloh, die wegen sehr schlechten Trinkens per Sonde gegeben werden mußten. Der toxische Zustand blieb unverändert bis 21. VIII., dann verfiel das Kind in tiefkomatösen Zustand und lebte die letzten 24 Stunden in voller Agonie. Die Temperatur schwankte zwischen 38 und $37^{\circ}$. 
Gewicht am 23. VIII. 3600 g. Exitus an demselben Tage um $9^{\text {h }}$ früh. Sektion verweigert.

Bei dem Kinde wurden zwei Blutuntersuchungen gemacht und zwar: 22. VIII. $4^{\text {h }}$ nachm. Sinuspunktion, keine Reaktion dabei, moribund.

23. VIII. $9^{\mathrm{h}}$ vorm. Herzpunktion.

$$
\begin{aligned}
& \text { Blut, aktuelle Reaktion . . . . . . . 6,96) }{ }_{\text {Breite }}^{\mathrm{CO}_{2} \text {-Regul }} \\
& \text { " } \left.\mathrm{CO}_{2} \text {-freies .......... . } 7,10\right\}=14 \%
\end{aligned}
$$

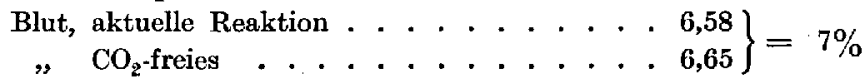

Ein Teil vơn dem Blute sowohl vom 22 . als auch vom 23. wurde zur Bestimmung der $\mathrm{O}_{2}$-Dissoziationskurve des Hämoglobins verwendet. Diese Untersuchungen schließen hier an.

\section{1. $\mathrm{O}_{2}$-Dissoziationskurve des Hämoglobins.}

22. VIII. 14 Sinuspunktion.

$$
\text { Punkt I. }
$$

2,0 ccm Blut im Tonometer, daselbst ununterbrochen $25 \mathrm{Min}$. bei $37,8^{\circ}$ geschüttelt.

$$
\left.\begin{array}{llr}
\text { Temp. : } 23^{\circ} & \text { Gasanalysen } & \mathrm{I}=3,24 \\
\text { Druck: } 752 \mathrm{~mm} \mathrm{Hg} & \text { aus dem Tonometer } & \mathrm{II}=3,37
\end{array}\right\}=3,3 \% \mathrm{O}_{2}
$$

Differentialmanometer bei $20^{\circ}$ im Wasserbade.

In die Birne ca. $0,1 \mathrm{ccm}$ Blut.

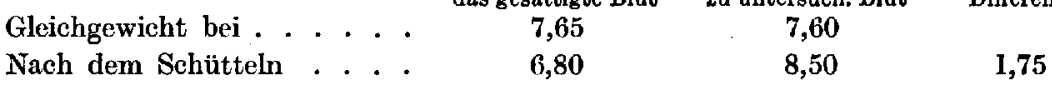

$$
\begin{aligned}
& \begin{array}{l}
\text { In die linke Birne } \\
\text { das geslittigte Blut }
\end{array} \begin{array}{c}
\text { In die rechte Birne das } \\
\text { zu untersuch. Blut }
\end{array} \text { Differenz } \\
& \text { Kaliumferricyanid rechts, } \\
& \begin{array}{llll}
\text { Hähne vorher nicht geöffnet } & 8,30 & 7,05 & 1,20
\end{array} \\
& \% \text { Sättigung }=40,7 \% \mathrm{O}_{2} \\
& \mathrm{O}_{2} \text {-Druck }=24,8 \mathrm{~mm} \mathrm{Hg} \\
& \mathrm{P}_{\mathrm{H}}=7,10
\end{aligned}
$$

$2,0 \mathrm{ccm}$ Blut im Tonometer im Wasserbade 25 Min. bei $37,8^{\circ}$ geschüttelt.
Temp.: $23^{\circ}$
Druck: $752 \mathrm{~mm} \mathrm{Hg}$
Gasanalysen
$\left.\begin{array}{r}\mathrm{I}=4,3 \\ \mathrm{II}=4,7\end{array}\right\}=4,5 \% \quad \mathrm{O}_{2}$

Differentialmanometer bei $20^{\circ}$ im Wasserbade.

In die Birne ca. $0,1 \mathrm{~cm}$ Blut.

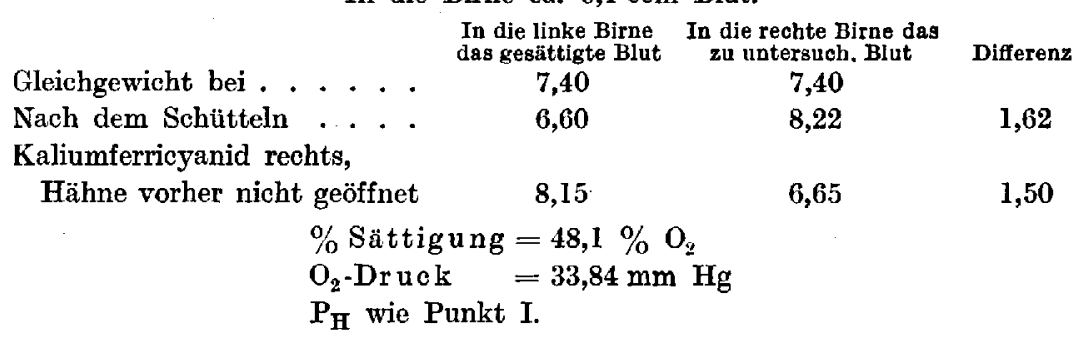


Diese Punkte sind zu einer Dissoziationskurve auf Fig. 23, I vereinigt.

Das Kind starb am nächsten Tage (23. VIII. 14). Die folgenden Bestimmungen zeigen die Verhältnisse der Sauerstoffbindung im Herzblute, das gleich nach dem Exitus entnommen warde.

23. VIII. 14.

$$
\text { 2. } \mathrm{O}_{2} \text {-Dissoziationskurve des Hä moglobins. }
$$

\section{Punkt I.}

$3,0 \mathrm{ccm}$ Herzblut im Tonometer, daselbst ununterbrochen 25 Min. bei $38^{\circ}$ geschüttelt.

Temp.: $20^{\circ}$

Druck: $753 \mathrm{~mm} \mathrm{Hg}$

$$
\left.\begin{array}{lr}
\text { Gasanalysen } & \mathrm{I}=1,22 \\
\text { aus dem Tonometer } & \mathrm{II}=1,30
\end{array}\right\}=1,3 \% \mathrm{O}_{2}
$$

A. Differentialmanometer I bei $17,5^{\circ} \mathrm{im}$ Wasserbade.

\begin{tabular}{|c|c|c|}
\hline $\begin{array}{l}\text { In die linke Birne } \\
\text { das gesättigte Blut }\end{array}$ & $\begin{array}{l}\text { In die rechte Birne das } \\
\text { zu untersuch. Blut }\end{array}$ & Differenz \\
\hline 7,68 & 7,64 & \\
\hline 6,88 & 8,41 & 1,57 \\
\hline 7,66 & 7,70 & $-0,08$ \\
\hline
\end{tabular}

In die Birne ca. $0,1 \mathrm{ccm}$ Blut.

Gleichgewicht bei .. . . .

Nach dem Schütteln . . . .

7,70

$-0,08$

B. Differentialmanometer II bei $17,5^{\circ}$ im Wasserbade.

In die Birne ca. 0,1 ccm Blut.

$$
\begin{aligned}
& \text { In die linke Birne In die rechte Birne das } \\
& \text { das gesāttigte Blut zu untersuch. Blut Differenz } \\
& 6,93 \quad 8,38 \\
& 8,44 \quad 6,97 \\
& \left.\% \text { Sättigung } \begin{array}{l}
\mathrm{A}=<0 \% \\
\mathrm{~B}=<0 \%
\end{array}\right\}=0 \% \mathrm{O}_{2} \\
& \mathrm{O}_{2} \text {-Druck . . . . . }=9,75 \mathrm{~mm} \mathrm{Hg} \\
& \mathrm{P}_{\mathrm{H}} \text {. . . . . . . }=6,65
\end{aligned}
$$

Gleichgewicht bei . . . . .

$7,68 \quad 7,64$

Kaliumferricyanid rechts,

Hähne vorher geöffnet

Punkt II.

$3,0 \mathrm{ccm}$ Herzblut, im Tonometer daselbst ununterbrochen $25 \mathrm{Min}$. bei $38^{\circ}$ geschüttelt.

Temp.: $20^{\circ}$

Druck: $753 \mathrm{~mm} \mathrm{Hg}$ $\left.\begin{array}{ll}\text { Gasanalysen } & \text { I }=2,80 \\ \text { aus dem Tonometer } & \text { II }=3,00\end{array}\right\}=2,9 \% \mathrm{O}_{2}$

A. Differentialmanometer I bei $17^{\circ} \mathrm{im}$ Wasserbade.

In die Birne ea. 0,1 ccm Blut.

Gleichgewicht bei ...... . In die linke Birne
das gesättigte Blut

In die rechte Birne das 7,66 zu untersuch. Blut

Nach dem Schütteln

8,30 7,61

Kaliumferricyanid rechts,

6,88

7,00

Differenz

Hähne vorher geöffnet

8,40

$\cdot$




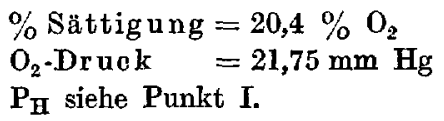

Punkt III.

$3,0 \mathrm{ccm}$ Herzblut im Tonometer, daselbst ununterbrochen 25 Min. bei $38^{\circ}$ geschüttelt.

$$
\left.\begin{array}{llr}
\text { Temp.: } 20^{\circ} & \text { Gasanalysen } & \mathrm{I}=5,7 \\
\text { Druck: } 753 \mathrm{~mm} \mathrm{Hg} & \text { aus dem Tonometer } & \mathrm{II}=5,4
\end{array}\right\}=5,6 \% \mathrm{O}_{2}
$$

Differentialmanometer bei $18^{\circ}$ im Wasserbade.

In die Birne ca, 0, 1 com Blut.

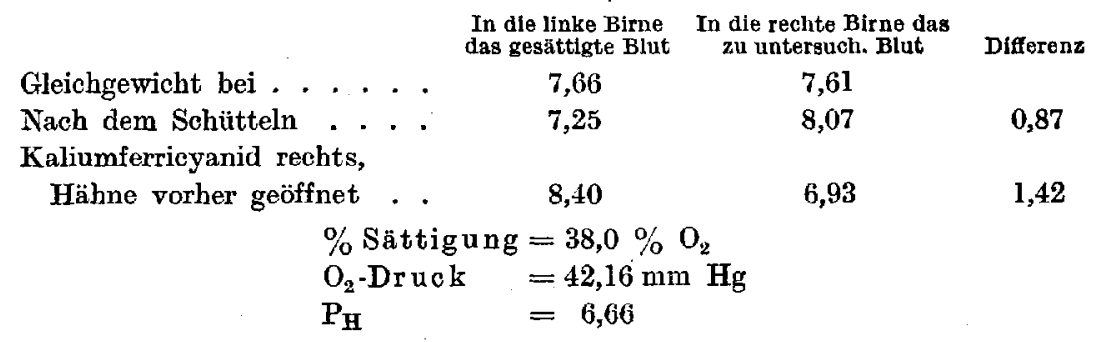

Aus der Gleichgewichtsformel wurden $\mathrm{n}$ und $\mathrm{K}$ berechnet:

$$
\begin{aligned}
\mathbf{n} & =1,5 \\
\mathbf{K} & =0,00279 .
\end{aligned}
$$

Mit Hilfe dieser Werte wurde durch die Gleichgewichtsformel Punkt IV bestimmt.

Punkt IV. $\quad \%$ Sättigung $=75,0 \% \mathrm{O}_{\mathfrak{s}}$

$$
\mathrm{O}_{\mathrm{z}} \text {-Druck }=100,0 \mathrm{~mm} \mathrm{Hg} \text {. }
$$

Diese Werte sind auf Fig. 23, II zu einer Dissoziationskurve vereinigt. Der IV. berechnete Punkt ist mit * bezeichnet.

Fall Nr. 84.

Pis kol, Wilhelm, 5 Mon., Intoxikation im Verlaufe von akuter sohwerer Ernährungsstörung. Am 7. VII. 14 im K.-A.-V.-Haus geboren, Geburtsgewicht $3030 \mathrm{~g}, 4$ Mon. Brust, dabei gutes Gedeihen, kommt in die Außenpflege, wo es $1 / 2$ Milch mit $5 \%$ Rohrzucker 5 mal $7-8^{\prime}$ bekam. Am 4. $X$. wird das Kind unruhig, will nicht mehr trinken. Stühle anfangs unverändert, seit dem 6. VII. aber dünn. An demselben Tage wurde es schläfrig, verweigerte die Nahrung ganz und gar und wurde am 7. X. in die Anstalt zurückgebracht. Status: Deutlich abgemagertes Kind, $3500 \mathrm{~g}$. schwer benommen. Stühle dünn, teils spritzend, Temperatur schwankt zwischen 38 und $37^{\circ}$, bekommt zuerst einen Tag Tee, dann kleine Mengen Frauenmilch, $180-300 \mathrm{~g}$ täglich und Tee $200-300 \mathrm{~g}$ dazu. Trank in den ersten Tagen sehr schlecht, vom 10. X, ab aber etwas besser. Das Kind exhält außerdem täglich $125-150 \mathrm{~g}$ Ringerlösung subcutan. Kein nennenswertes Erbrechen, Stühle schleimig, zahlreich. Die Benommenheit verschwand allmählich, am 11. X. war das Sensorium ganz klar, das Kind fixierte, war nur auffallend schlaff. Lungen und Herz $\mathrm{O}$. B., die Fiebererscheinungen verschwanden 
erst nach 2 Wochen und die Stühle wurden allmählich gut. Das Kind blieb längere Zeit im Hause, entwickelte sich nachher leidlich und wurde im Alter von 11 Mon. mit einem Gewicht von $6300 \mathrm{~g}$ entlassen.

Folgende Bestimmungen wurden vorgenommen:

9. X. I. Blutentnahme, leicht benommen, doch lebhafte Reaktion dabei, die Aciditätswerte und die Dissoziationskurve später.

Lumbalpunktion, Druck niedrig.

$$
\begin{aligned}
& \text { Liquor klar . . . . . . . . . . . 7,99 } \\
& \text { Urin . . . . . . . . . . 6,68 }
\end{aligned}
$$

12. X. 2. Blutentnahme, nicht mehr benommen, die Werte siehe unten.

9. X. 1. Blutentnahme, leicht benommen.

Blut, aktuelle Reaktion . .

" $\mathrm{CO}_{2}$-freies ........... 7,98$\}=32 \%$

1. $\mathrm{O}_{2}$-Dissoziationskurve des Hämoglobins.

\section{Punkt I.}

2,0 ccm Herzblut im Tonometer, daselbst ununterbrochen $25 \mathrm{Min}$. bei $38^{\circ}$ geschüttelt.

Temp.: $19^{\circ}$

Druck: $760 \mathrm{~mm} \mathrm{Hg}$

$$
\left.\begin{array}{lr}
\text { Gasanalysen } & \mathrm{I}=3,5 \\
\text { aus dem Tonometer } & \mathrm{II}=3,1
\end{array}\right\}=3,3 \% \mathrm{O}_{\mathbf{2}}
$$

A. Differentialmanometer $\mathrm{I}$ bei $17^{\circ}$ im Wasserbade.

In die Birne ca. 0,1 cem Blut.

$\begin{array}{ccc}\begin{array}{c}\text { In die linke Birne } \\ \text { das gesättigte Blut }\end{array} & \begin{array}{c}\text { In die rechte Birne das } \\ \text { zu untersuch. Blut }\end{array} & \text { Differenz } \\ 7,65 & 7,60 & \\ 7,27 & 8,00 & 0,78 \\ & & \\ 8,60 & 6,65 & 1,90\end{array}$

Gleichgewicht bei . . . .

B. Differentialmanometer II bei $17^{\circ}$ im Wasserbade.

In die Birne ca. $0,1 \mathrm{ccm}$ Blut.

In die linke Birne In die rechte Birne das
das gesáttigte Blut

Gleichgewicht bei . . . $7,40 \quad 7,40$

$\begin{array}{llll}\text { Nach dem Schütteln . . . } & 7,07 & 7,88 & 0,81\end{array}$

Kaliumferricyanid rechts,

$\begin{array}{llll}\text { Hähne vorher nicht geöffnet } & 8,40 & 6,28 & 2,12\end{array}$

$$
\begin{gathered}
\text { \% Sättigung } \left.\begin{array}{r}
A=70,9 \\
B=72,3
\end{array}\right\}=71,6 \% \mathrm{O}_{2} \\
\mathrm{O}_{2} \text {-Druck . . . . . . . . . . . . }=25,0=7,98 \\
\mathrm{P}_{\mathrm{H}} \cdot . . \mathrm{Hg} \\
\text { Punkt II. }
\end{gathered}
$$

2,0 cem Herzblut im Tonometer, daselbst ununterbrochen 25 Min. bei $38^{\circ}$ geschüttelt. 
und Intoxikationsacidosis in ihren Beziehungen zueinander.

$\left.\begin{array}{llr}\text { Temp.: } 19^{\circ} & \text { Gasanalysen } & \mathrm{I}=2,4 \\ \text { Druck : } 760 \mathrm{~mm} \mathrm{Hg} & \text { aus dem Tonometer } & \mathrm{II}=2,2\end{array}\right\}=2,3 \% \mathrm{O}_{2}$

Differentialmanometer I bei $17^{\circ}$ im Wasserbade.

In die Birne ca. $0,1 \mathrm{com}$ Blut.

Gleichgewicht bei ......

In die linke Birne In die rechte Birne das das gesättigte Blut zu untersuch. Blut

Differenz

Nach dem Schütteln . . .

$\begin{array}{ll}7,65 & 7,60 \\ 6,60 & 8,63\end{array}$

8,63

2,08

Kaliumferricyanid rechts,

Hähne vorher nicht geöffnet

$8,50 \quad 6,80 \quad 1,65$

$$
\begin{aligned}
& \% \text { Sättigung }=44,2 \% \mathrm{O}_{2} \\
& \mathrm{O}_{2} \text {-Druck }=17,48 \mathrm{~mm} \mathrm{Hg} \\
& \mathbf{P}_{\mathbf{H}}=7,98 .
\end{aligned}
$$

12. X. 14. 2. Blutentnahme, sehr schlaff, nicht mehr benommen.

$$
\begin{aligned}
& \text { Blut, aktuelle Reaktion . . . . . . } 7,45)_{\text {Breite }}^{\text {CO. }_{\text {Begul. }}} \\
& \text { " } \left.\mathrm{CO}_{2} \text { freies ......... 8,02 }\right\}=57 \%
\end{aligned}
$$

2,0 cem Herzblut im Tonometer, daselbst ununterbrochen $25 \mathrm{Min}$. bei $38^{\circ}$ geschüttelt.

$$
\left.\begin{array}{llr}
\text { Temp.: } 20^{\circ} & \text { Gasanalysen } & \mathrm{I}=2,0 \\
\text { Druck: } 754 \mathrm{~mm} \mathrm{Hg} & \text { aus dem Tonometer } & \mathrm{II}=1,9
\end{array}\right\}=2,0 \% \mathrm{O}_{2}
$$

A. Differentialmanometer I bei $17^{\circ}$ im Wasserbade.

In die Birne ca. $0,1 \mathrm{ccm}$ Blut.

Gleichgewicht bei ......

In die linke Birne In die rechte Birne das das gesättigte Blut zu untersuch. Blut

Differenz

Nach dem Schïtteln ....

7,62

7,20

7,58

8,00

0,84

Kaliumferrieyanid rechts,

Hähne vorher nicht geöffnet

8,50

6,75

1,71

B. Differentialmanometer II bei $17^{\circ}$ im Wasserbade.

In die Birne ca. $0,1 \mathrm{~cm}$ Blut.

In die linke Birne In die rechte Birre das

das gesalttigte Blut $\begin{gathered}\text { 2u untersuch. Blut } \\ \text { differenz }\end{gathered}$

Gleichgewicht bei . . . .

$\begin{array}{lll}\mathbf{7 , 4 0} & \mathbf{7 , 4 4} & \\ \mathbf{6 , 8 4} & \mathbf{7 , 8 7} & 0,99\end{array}$

Nach dem Schütteln . . . $\quad 6,84$

8,30

6,42

1,92

Hähne vorher nicht geöffnet

$$
\begin{aligned}
& \text { \% Sättigung } \left.\begin{array}{l}
\mathrm{A}=67,0 \\
\mathrm{~B}=66,0
\end{array}\right\}=66,5 \% \mathrm{O}_{2} \\
& \mathrm{O}_{2}-\text { Druck . . . . . }=15,08 \mathrm{~mm} \mathrm{Hg} \\
& \mathrm{P}_{\mathrm{H}} \text {......... . . }=8,02
\end{aligned}
$$


Punkt II.

2,0 ccm Herzblut im Tonometer, daselbst ununterbrochen 25 Min. bei $38^{\circ}$ geschüttelt.
Temp.: $20^{\circ}$
Gasanalysen
Druck: $754 \mathrm{~mm} \mathrm{Hg}$
aus dem Tonometer

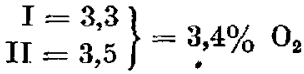

A. Differentialmanometer I bei $17^{\circ}$ in Wasserbade.

In die Birne ca. 0, $1 \mathrm{ccm}$ Blut.

In die linke Birne In die rechte Birne das

das gesättigte Blut zu untersuch. Blut Differenz

Gleichgewicht bei . . . . . 7,59

7,55

$7,46 \quad 7,80$

Kaliumferricyanid rechts,

Hähne vorher nicht geöffnet

8,94

6,36

B. Differentialmanometer II bei $17^{\circ}$ im Wasserbade.

In die Birne ca. 0,1 cem Blut.

Gleichgewicht bei . . . . .

In die linke Birne In die rechte Birne das das gesättigte Blut zu untersuch. Blut

Differenz

Nach dem Schütteln . . . $\quad 7,20$

$7,42 \quad 7,44$

$7,20 \quad 7,52$

Kaliumferricyanid rechts,

$\begin{array}{llll}\text { Hähne vorher nicht geöffnet } & 8,40 & 6,33 & 2,09\end{array}$

$$
\begin{aligned}
& \text { \% Sättigung } \left.\begin{array}{l}
A=87,0 \\
B=87,5
\end{array}\right\}=87,25 \% \mathrm{O}_{2} \\
& \mathrm{O}_{2} \text {-Druck . . . . . }=25,64 \mathrm{~mm} \mathrm{Hg} \\
& \mathrm{P}_{\mathbf{H}} . . . \\
& . . . . .
\end{aligned}
$$

Mit Hilfe der obigen Zahlen wurden die Werte $\mathbf{n}$ und $\mathrm{K}$ in der Gleichgewichtsformel berechnet:

$$
\begin{aligned}
\mathbf{K} & =0,00304 \\
\mathbf{n} & =\mathbf{2 , 4}
\end{aligned}
$$

und danach durch dieselbe Formel ein 3. Punkt auf der Dissoziationskurve rechnerisch bestimmt, und zwar

$$
\text { Punkt III } \quad\left\{\begin{array}{l}
\% \text { Sättigung }=96,7 \% \mathrm{O}_{2} \text { bei } \\
\mathrm{O}_{2} \text {.Druck }=50,0 \mathrm{~mm} \mathrm{Hg}
\end{array}\right.
$$

Aus den obigen Zahlen gebaute Dissoziationskurven vom 9. $\mathrm{X}$, und 12. $\mathrm{X}$. sind auf der Fig. 23, I und II S. 328 graphisch dargestellt.

\section{Fall Nr. 85.}

Bueter, Kurt, 10 $/ 2$ Mon., Intoxikation im Verlaufe von akutem Brechdurchfall. Am 20. X. 13 geb., Geburtsgewicht unbekannt, stammt aus Familie, wo Polymortalität, von 13 Kindern 9 in den ersten Monaten an Brechdurchfall zugrunde gegangen. Das Kind bereits zweimal im K.-A.V.-Haus gewesen, das erste Mal 2 Tage alt wegen Krämpfen, das zweite Mal im Alter von 3 Mon. wegen Atrophie und Bronchopneumonie; nachher bis zu dieser Erkrankung auffallend 
gut zugenommen. Ernährung zuletzt $1 / 2$ Milch 4 mal $12^{\prime}$ und daneben 2 mal Grießbrei und Gemüse. Am 7. IX. plötzlich unruhig, heftiges Frbrechen und dünne Stühle, am 8. IX. gegen Abend etwas schläfrig, wollte nicht mehr trinken, in der Nacht zum 9. IX. Krämpfe, deswegen und auch wegen der bestehenden dünnen Stühle und des Erbrechens 9. IX. 14 zur Aufnahme gebracht. Status: Sehr kräftiges, dickes, wasserverarmtes Kind. Schwer toxisch. Pulslos. Cornea leicht trübe. Ringerlösung subcutan, Campher, trank noch allein etwas Tee; Lungen o. B. Um $5^{\text {h }}$ nachm. plötzlich Exitus.

Herzpunktion und Sektion gleich danach. Sektion ergab: Oedema pulmonis dextra, Bronchitis lat. ambor, Degeneratio adiposa hepatis, Hyperplasio des ganzen Lymphdrüsensystems; Magen- und Darmkanal zeigten keine besonderen Veränderungen, Injektion oder dergleichen.

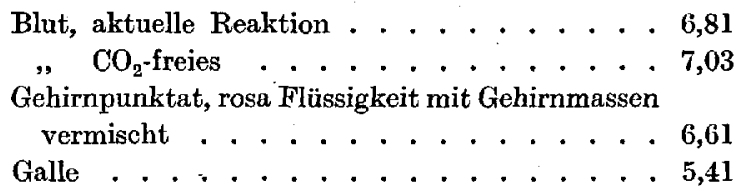

Ein Teil vom Herzblut wurde zur Bestimmung der Dissoziationskurve verwendet. Ich lasse die Bestimmungen folgen:

$$
\begin{gathered}
\mathrm{O}_{2} \text {-Dissoziationskurve des Hämoglobins. } \\
\text { Punkt I. }
\end{gathered}
$$

$3,0 \mathrm{ecm}$ Herzblut im Tonometer, daselbst ununterbrochen 25 Min. bei $37,8^{\circ}$ geschüttelt.

Temp.: $23^{\circ}$

Druck: $752 \mathrm{~mm} \mathrm{Hg}$

$$
\left.\begin{array}{lr}
\text { Gasanalysen } & \mathrm{I}=3,24 \\
\text { aus dem Tonometer } & \text { II }=3,37
\end{array}\right\}=3,3 \% \mathrm{O}_{2}
$$

\begin{tabular}{|c|c|c|c|}
\hline Gleichgewicht bei . . . . & $\begin{array}{c}\text { In die linke Birne } \\
\text { das gesättigte Blut } \\
7,65\end{array}$ & $\begin{array}{c}\text { In die rechte Birne } \\
\text { das zu untersuch. Blut } \\
7,60\end{array}$ & Differenz \\
\hline Nach dem Schütteln & 6,80 & 8,50 & 1,75 \\
\hline \multicolumn{4}{|l|}{ Kaliumferricyanid rechts, } \\
\hline Hähne vorher nicht geöffnet. & 8,30 & 7,05 & 1,20 \\
\hline $\begin{array}{l}\% \text { Sättig } \\
\mathrm{O}_{2} \text {-Druc } \\
\mathrm{P}_{\mathbf{H}}\end{array}$ & $\begin{aligned} \mathrm{ung} & =40,7 \% \mathrm{O} \\
& =24,8 \mathrm{~mm} \\
& =7,03\end{aligned}$ & & \\
\hline
\end{tabular}

Differentialmanometer bei $20^{\circ}$ im Wasserbade.

In die Birne ca. $0,1 \mathrm{ccm}$ Blut.

Punkt II.

3,0 com Herzblut im Tonometer, daselbst ununterbrochen 25 Min. bei $37,8^{\circ}$ geschüttelt.

Temp.: $23^{\circ}$

Druck: $752 \mathrm{~mm} \mathrm{Hg}$
Gasanalysen

aus dem Tonometer

$$
\left.\begin{array}{r}
I=4,3 \\
I I=4,7
\end{array}\right\}=4,5 \% \quad O_{2}
$$


Differentialmanometer bei $20^{\circ}$ im Wasserbade.

In die Birne ca. $0,1 \mathrm{ccm}$ Blut.

In die linke Birne In die rechte Birne

das gesättigte Blut das zu untersuch. Blut Differenz

Gleichgewicht bei . .....

$\begin{array}{lr}7,40 & \mathbf{7 , 4 0}\end{array}$

Nach dem Schütteln ....

6,60

8,22

1,62

Kaliumferricyanid rechts,

Hähne vorher nicht geöffnet

8,15

6,65

1,50

$$
\begin{array}{ll}
\% \text { Sättigung } & =48,1 \% \mathrm{O}_{2} \\
\mathrm{O}_{\mathbf{2}} \text {-Druck } & =33,8 \mathrm{~mm} \mathrm{Hg} \\
\mathbf{P}_{\mathbf{H}} & =7,01
\end{array}
$$

Vereinigt man die obigen Punkte zu einer Dissoziationskurve, so bekommt man eine Linie, die einen auffallend niedrigen Verlauf hat, aber doch etwas höheren als beim Fall 83 (Kind Müller).

Fall Nr. 86.

Behrendt, Charlotte, 3 Mon. alt, Intoxikation im Verlaufe von chronischer Ernährungsstörung. Am 9. V. 14 geb., ausgetragenes Kind, von Anfang an künstlich ernährt. Oft etwas dünne Stühle, deswegen nur $1 / 2$ Milch-Nahrung in den letzten Zeiten mit Plasmonzusatz. Trotz der dünnen Stühle bis vor 8 Tagen regelmäßig zugenommen. Am 5. VIII. fing das Kind an zu stöhnen, die Stühle wurden noch dünner, jede Windel voll, hustete stark, hatte am folgenden Tage Erbrechen und sohlechten Appetit, nahm stark ab. Am 12. VIII. sehr schläfrig, nur ganz wenig getrunken, heute am 13. VIII. gar nichts mehr getrunken, bewußtlos, deswegen zur Aufnahme gebracht. Status: Stark abgemagertes Kind, 3400 g Gewicht, stark wasserverarmt, Soor, reichliches Knisterrasseln über beiden Unterlappen, links hinten unten leichtes Bronchialatmen und leichte Dämpfung. Bekommt am ersten Tage nur Tee und Ringerlösung $100 \mathrm{ccm}$ subcutan, am folgenden Tage neben Tee $50 \mathrm{~g}$ Frauenmilch. In den nächsten Tagen steigt die Frauenmilchmenge täglich um $50 \mathrm{~g}$ bis $450 \mathrm{~g}$. Späterhin wurde neben Frauenmilch 1/2 Milch (Larosan) gegeben. Das Kind war deutlich benommen rom 13. bis 15. VIII, am 16. fing das Sensorium an allmählich klar zu werden und am 17. VIII. merkte man nichts mehr von Benommenheit, fixierte auch schon. Die Dämpfung links hinten unten verschwand allmählich, die Temperatur blieb vom 13. bis 22. dauernd hoch remittierend, von $37,4^{\circ}$ bis $39^{\circ}$, seitdem Monothermie um ca. $37^{\circ}$. Das Kind wurde am 18. IX. mit $4120 \mathrm{~g}$ geheilt entlassen.

Folgende Untersuchungen wurden vorgenommen:

15. VIII. $12^{\mathrm{h}}$ mittags Sinuspunktion, benommen, reagiert doch mit Schreien.

Blut, aktuelle Reaktion . . . . . . . . 7,46

17. VIII. $12^{\mathrm{h}}$ mittags Sensorium klar, fixiert schon, aber noch sehr müde.

$$
\begin{aligned}
& \mathrm{CO}_{\mathrm{g}} \text {-Regul.- }
\end{aligned}
$$

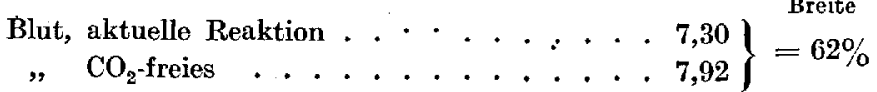

Aus demselben Blute wurde ein Teil zur Bestimmung der Dissoziationskurve benutzt, deren Werte folgen: 
$\mathrm{O}_{2}$-Dissoziationskurve des Hämoglobins.

17. VIII. 14, $12^{\mathrm{h}}$ mittags Sinuspunktion.

\section{Punkt I.}

2,0 cem Blut im Tonometer, daselbst ununterbrochen $25 \mathrm{Min}$. bei $37,4^{\circ}$ geschüttelt.
Temp.: $19^{\circ}$
Druck: $760 \mathrm{~mm} \mathrm{Hg}$
Gasanalysen
$\left.\begin{array}{r}\mathrm{I}=3,3 \\ \mathrm{II}=3,4\end{array}\right\}=3,35 \% \quad \mathrm{O}_{2}$

A. Differentialmanometer I béi $18^{\circ} \mathrm{im}$ Wasserbade.

In die Birne ca. $0,1 \mathrm{ccm}$ Blut.

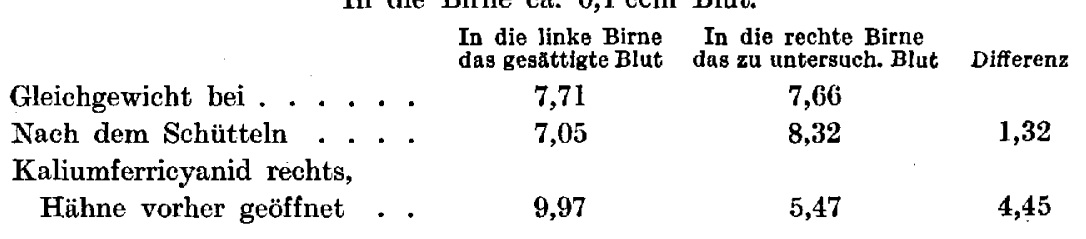

B. Differentialmanometer II bei $18^{\circ}$ im Wasserbade.

In die Birne ca. $0,1 \mathrm{ccm}$ Blut.

\begin{tabular}{|c|c|c|c|c|}
\hline & & $\begin{array}{l}\text { In die linke Birne } \\
\text { das gesättigte Blut }\end{array}$ & $\begin{array}{l}\text { In die rechte Birne } \\
\text { das zu untersuch. Blut }\end{array}$ & Differenz \\
\hline Gleichgewicht bei & . . & 7,50 & 7,46 & \\
\hline Nach dem Schütteln & $0^{\circ}$ & 7,05 & 7,90 & 0,89 \\
\hline \multicolumn{5}{|l|}{ Kaliumferricyanid rechts, } \\
\hline Hähne vorher geöffnet & - · & 9,25 & 5,65 & 3,56 \\
\hline 0 & $\begin{array}{l}\text { Sätt } \\
\text {-Dru } \\
\text {. . }\end{array}$ & $\left.\begin{array}{r}\mathrm{A}=70,3 \\
\mathrm{~B}=74,3\end{array}\right\}$ & $\begin{array}{l}=72,3 \% \mathrm{O}_{2} \\
=25,5 \mathrm{~mm} \mathrm{Hg} \\
=7,92\end{array}$ & \\
\hline
\end{tabular}

Dieser Doppelpunkt ist auf der Dissoziationskurve Fig. 23 mit $\times$ bezeichnet. Ihm entsprechende Dissoziationskurve würde einen verhältnismäßig hohen Verlauf annehmen, annähernd denselben wie die Kurve des Falles Nr. 84 (Piskol, Fig. 23, I) während des toxischen Stadiums.

\section{Fall Nr. 87.}

Otto, Erich, 4 Mon. alt, schwere Atrophie bei chronisch ernährungsgestörtem, exsudativem Kinde. Geb. 28. III. 14, Geburtsgewicht $3270 \mathrm{~g}$. 4 Wochen lang Muttermilch, dann 6 Wochen Mehlsuppe mit etwas Milch; bekam Durchfall, deswegen IMon. Amme, dann Backhausmilch. Andauernd langsam abgenommen, Stühle zeitweise hart, zeitweise dünner, nie Erbrechen. Seit dem ersten Lebensmonate Schorf und Ekzem am ganzen Körper. Aufnahme am 4. VIII. 14. Status: Blasses, leicht spastisches, stark atrophisches Kind, macht elenden und schwachen Eindruck. Gewicht $3100 \mathrm{~g}$, Alter 4 Mon. Die ganze Haut stark gerötet, an manchen Stellen wund, stark abschilfernd. Starke Untertemperatur. Herz, Lungen o. B. Sensorium frei. Stühle weißlich, salbig. Bekommt $450-600 \mathrm{~g}$ täglich $1 / 2$-Milch (Larosan) mit 5\% Rohrzucker, trinkt leidlich, Gewebsturgor und Allgemeinbefinden schienen schon etwas besser. Am 10. VIII. $8^{\mathrm{h}}$ morgens plötzlich Exitus. 10. VIII. Herzpunktion gleich nach dem Exitus.

Blut, aktuelle Reaktion . . . . . . . 7,21 
Außerdem wurde ein Teil dieses Blutes zur Bestimmung der Dissoziationskurve benutzt, wie aus dem Folgenden ersichtlich:

$$
\begin{gathered}
\mathrm{O}_{2} \text {-Dissoziationskurve des Blutes. } \\
\text { Punkt I. }
\end{gathered}
$$

$2,0 \mathrm{ccm}$ Herzblut im Tonometer, daselbst ununterbrochen 25 Min. bei $38^{\circ}$ geschüttelt.

Temp.: $22^{\circ}$

Druck: $753 \mathrm{~mm} \mathrm{Hg}$

$\left.\begin{array}{lr}\text { Gasanalysen } & \mathrm{I}=3,55 \\ \text { aus dem Tonometer } & \mathrm{II}=3,55\end{array}\right\}=3,55 \% \quad \mathrm{O}_{2}$

A. Differentialmanometer I bei $21^{\circ} \mathrm{im}$ Wasserbade.

In die Birne ca. 0,1 ccm Blut.

In die linke Birne In die rechte Birne

das gesattigte Blut das zu untersuch. Blut Differenz

Gleichgewicht bei ......

$\begin{array}{lll}7,72 & 7,67 & \\ 7,38 & 8,00 & 0,67\end{array}$

Nach dem Schütteln .... 7,38

8,00

0,67

Kaliumferricyanid rechts,

Hähne vorher geöffnet . . $\quad 9,00 \quad 6,44 \quad 2,51$

B. Differentialmanometer II bei $21^{\circ} \mathrm{im}$ Wasserbade.

In die Birne ca. 0,1 cem Blut.

Gleichgewicht bei .....

In die linke Birne. In die rechte Birne

das gesättigte Blut das zu untersuch. Blut Differenz

$\begin{array}{llll}\text { Nach dem Sehütteln . . . } & 7,18 & 7,80 & 0,62\end{array}$

Kaliumferricyanid rechts,

$\begin{array}{llll}\text { Hähne vorher geöffnet } \ldots & 8,72 & 6,22 & 2,50\end{array}$

$$
\begin{aligned}
& \left.\begin{array}{cc}
\% \text { Sattigung } & \begin{array}{c}
\mathrm{A}=73,3 \\
,
\end{array} \\
\mathrm{~B}=75,2
\end{array}\right\}=74,3 \% \quad \mathrm{O}_{2} \\
& \mathrm{O}_{2} \text {-Druck ..... }=26,7 \mathrm{~mm} \mathrm{Hg}
\end{aligned}
$$

2,0 ccm Herzblut im Tonometer, daselbst ununterbrochen 25 Min. bei $38^{\circ}$ geschüttelt.

Temp. : $23^{\circ}$

Druck: $753 \mathrm{~mm} \mathrm{Hg}$

$\left.\begin{array}{lr}\text { Gasanalysen } & \mathrm{I}=3,5 \\ \text { aus dem Tonometer } & \mathrm{II}=3,1\end{array}\right\}=3,3 \% \quad \mathrm{O}_{2}$

A. Differentialmanometer I bei $21^{\circ}$ im Wasserbade.

In die Birne ca. 0,1 ccm Blut.

Gleichgewicht bei . . . . .

In die linke Birne

das geslttigte Blut.

Naoh dem Schütteln ... 7,28

7,73
7,28

9,18

In die rechte Birne

das zu untersuch. Blut Differenz

Kaliumferricyanid rechts,

Hähne vorher geöffnet · .

B. Differentialmanometer II bei $21^{\circ}$ im Wasserbade.

8,15

0,92

In die Birne ca. 0,1 cem Blut.

In die linke Birne In die rechte Birne

Gleichgewicht bei . . . . . das gesättigte Blut

das zu untersuch. Blut Differenz

Nach dem Schütteln ....

7,48

7,08

7,91

0,85 
Kaliumferricyanid rechts,

Hähne vorher geöffnet

$$
8,95
$$

6,00

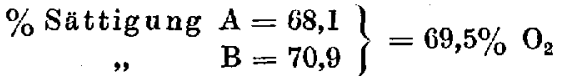

$$
\begin{aligned}
& \mathrm{O}_{2} \text {-Druck . . . . . }=24,8 \mathrm{~mm} \mathrm{Hg} \text {. }
\end{aligned}
$$

Vereinigt man diese Punkte zu einer Dissoziationskurve, so bekommt man eine Linie, die einen auffallend hohen Verlauf hat, annähernd gleich hoch wie im Falle 90 (Kind Maier, Helene). Siehe Fig. 24, I S. 330.

$$
\text { Fall Nr: } 88 .
$$

Werda, Wally, 101/4 Mon. alt, Lues congenita, chronische Ernährungsstörung. Am 10. X. 13 geb., Geburtsgewicht $1500 \mathrm{~g}$, aus einer Familie, wo von 7 Kindern 3 in den ersten Monaten gestorben sind. Von Anfang an künstlich ernährt, hat wegen mehrmaligen Auftretens von dünnen Stühlen aus der Fürsorgestelle längere Zeit Eiweißmilch bekommen, in der übrigen Zeit $1 / 2^{-}$bis ${ }^{2} / 3^{-M i l c h ~}$ mit $5 \%$ Rohrzucker. Hat trotz der dünnen Stühle leidlich zugenommen, schnieft seit der Geburt, in der Fürsorge mit Sublimatinjektionen wegen Lues congenita behandelt und wird von dort zu weiterer Behandlung überwiesen. 17. VIII. 14 Status: Leidlich ernährtes Kind, Gewicht $5500 \mathrm{~g}$, mit deutlichem Caput natiforme und Sattelnase, starkem Schniefen, Milztumor; Lunge: links hinten unten leichte Schallabschwächungen mit zahlreichem Rasseln. Stühle leicht breiig, Urin: Eiweiß +, Zucker -, im Sediment granulierte Cylinder. Bekommt bei uns $1 / 2$-Milch (Larosan) und 3\% Zucker; macht schwachen Eindruck, Sensorium ist frei, Temperatur um $38^{\circ}$. Unerwarteter Exitus am 20. VIII. $6^{\mathrm{h}} 30^{\prime}$ morgens.

20. VIIT. Herzpunktion und Gehirnpunktion gleich danach.

Blut, aktuelle Reaktion . . . . . . . . 6,91

" $\mathrm{CO}_{2}$-freies . . . . . . . . 7,33

Gehirnpunktat mit Gehirnmassen vermischt . . 6,78

Cerebrospinalflüssigkeit aus Lumbalkanal mit

Spritze entnommen . . . . . . . 7,27

Außerdem wurde ein Teil des Herzblutes zur Bestimmung der Dissoziationskurve benutzt. Ich lasse die Werte folgen:

20. VIII. 1914 .

$$
\mathrm{O}_{2} \text {-Dissoziationskurve des Hämoglobins. }
$$

\section{Punkt I.}

$3,0 \mathrm{ccm}$ Herzblut im Tonometer, daselbst ununterbrochen 25 Min. bei $38^{\circ}$ geschüttelt.

Temp.: $20^{\circ}$

Druck: $752 \mathrm{~mm} \mathrm{Hg}$

$$
\left.\begin{array}{lr}
\text { Gasanalysen } & \mathrm{I}=2,38 \\
\text { aus dem Tonometer } & \mathrm{II}=2,50
\end{array}\right\}=2,4 \% \mathrm{O}_{2}
$$

Differentialmanometer bei $18^{\circ}$ im Wasserbade.

In die Birne ca. $0,1 \mathrm{ccm}$ Blut.

$\begin{array}{lccccc} & & & \begin{array}{c}\text { In die linke Birne } \\ \text { das gesăttigte Blut }\end{array} & \begin{array}{c}\text { In die rechte Birne } \\ \text { das zu untersuch. Blut }\end{array} & \text { Differenz } \\ \text { Gleichgewicht bei . . . . . . } & 7,66 & 7,60 & \\ \text { Nach dem Schütteln } & \text {. } & . & \mathbf{7 , 3 6} & \mathbf{7 , 9 3} & 0,63\end{array}$


Kaliumferricyanid rechts,

Hähne vorher nicht geöffnet

$$
8,24
$$

$$
\begin{aligned}
& \% \text { Sättigung }=42,7 \% \mathrm{O}_{2} \\
& \mathrm{O}_{2} \text {-Druck }=18,04 \mathrm{~mm} \mathrm{Hg} \\
& \mathrm{P}_{\mathbf{H}}=7,41 .
\end{aligned}
$$

Punkt II.

3,0 ccm Herzblut im Tonometer, daselbst ununterbrochen 25 Min. bei $37,6^{\circ}$ geschüttelt.

\begin{tabular}{|c|c|c|c|}
\hline & $\begin{array}{l}\text { In die linke Birne } \\
\text { das gesalttigte Blut }\end{array}$ & $\begin{array}{l}\text { In die rechte Birne } \\
\text { das zu untersuch. Blut }\end{array}$ & Differenz \\
\hline Gleichgewicht bei & 7,66 & 7,61 & \\
\hline Nach dem Schütteln & 7,63 & 7,91 & 0,33 \\
\hline \multicolumn{4}{|l|}{ Kaliumferricyanid rechts, } \\
\hline Hähne vorher nicht geöffnet & 8,60 & 7,70 & 0,85 \\
\hline $\begin{array}{l}\% \text { Sättig } \\
\mathrm{O}_{2}-\mathrm{Druc} \\
\mathrm{P}_{\mathrm{H}}\end{array}$ & $\begin{aligned} \mathrm{ng} & =61,1 \% \\
& =31,6 \mathrm{~mm} \\
& =7,33 .\end{aligned}$ & ${ }_{2}^{2}$ & \\
\hline
\end{tabular}

$$
\left.\begin{array}{llr}
\text { Temp: }: 20^{\circ} & \text { Gasanalysen } & I=4,20 \\
\text { Druck: } 752 \mathrm{~mm} \mathrm{Hg} & \text { aus dem Tonometer } & \text { II }=4,27
\end{array}\right\}=4,2 \% \mathrm{O}_{2}
$$

Differentialmanometer bei $18^{\circ}$ im Wasserbade.

In die Birne ca. $0,1 \mathrm{~cm}$ Blut.

Vereinigt man diese Punkte zu einer Dissoziationskurve, so nimmt sie einen deutlich niedrigen Verlauf, aber lange nicht so niedrigen wie bei Intoxikationen, z. B. Fall Nr. 83 (Kind Müller).

\section{Fall Nr. 89.}

Goerke, Johann, 5 Mon., Chronische Bronchopneumonie, Kopfphlegmone mit anschließendem Intoxikationszustand. Am 25. III. 14. geb., Geburtsgewicht unbekannt. 3 Mon. lang Brust, dabei verhältnismäßig gutes Gedeihen, dann Ziegenmilch mit etwas Wasser verdünnt, 6 mal $8^{\prime}$. - Hustete vom 3. Lebensmonate an und hatte röchelndes Atmen. Am 14. VIII. wurden die Stühle sehr dünn, der Husten schlimmer, anfangs kein Erbrechen, am 18. heftiges Erbrechen, am 19, verweigerte das Kind die Nahrung, am 20. in die Klinik gebracht. Status: Dem Alter entsprechend langes, mageres, leidlich ernährtes Kind mit sehr trockener Haut. Rechts unten über dem ganzen Unterlappen deutliche Dämpfung mit bronchialem Atemgeräusch. Aus dem rechten Ohr eiterige Sekretion, am Hinterkopf große phlegmonöse Furunkel. Stïhle zerfahren, Urin: Eiweiß +, Zucker -, im Sediment einzelne Zylinder, Sensorium leicht apathisch. Bekommt kleine Mengen Frauenmilch von $50-260 \mathrm{~g}$, außerdem reichlich Tee, $400-500 \mathrm{~g}$ täglich. Kein Erbrechen, trinkt leidlich und ist am 20. VIII. wieder klar. Untertemperatur nur am 24. VIII. bis $38^{\circ}$. Die phlegmonöse Infiltration am Hinterkopf breitete sich aus bis tief in den Nacken himunter, das Ohr eiterte reichlich und die Dämpfung überzog die ganze rechte Lunge. Am 25. VIII. wurde das Kind wieder sehr apathisch und am 26. VIII. erfolgte der Exitus nach langer Agonie. 
26. VIII. $10^{\text {h }}$ vorm. Herzpunktion und Sektion gleich nach dem Tode. Sektionsbefund: Bronchopneumonie bilateralis, Otitis media, Hyperplasia lienis, Phlegmone am Hinterkopf.

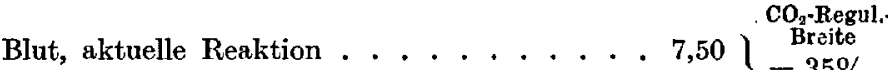

$$
\begin{aligned}
& \text { " } \left.\mathrm{CO}_{\mathrm{2}} \text {-freies . . . . . . . . . . . . } 7,85\right\}=35 \%
\end{aligned}
$$

Aus dem Herzblute wurde außerdem noch die Dissoziationskurve wie folgt bestimmt:

$$
\begin{gathered}
\mathrm{O}_{2} \text {-Dissoziationskurve des } \mathrm{Hämoglobins.} \\
\text { Punkt } \mathrm{I} \text {. }
\end{gathered}
$$

$3,0 \mathrm{ccm}$ Herzblut im Tonometer, daselbst ununterbrochen $25 \mathrm{Min}$. bei $37,8^{\circ}$ geschüttelt.

Temp.: $21^{\circ}$

Druck: $750 \mathrm{~mm} \mathrm{Hg}$

$$
\left.\begin{array}{lr}
\text { Gasanalysen } & \mathrm{I}=3,5 \\
\text { aus dem Tonometer } & \mathrm{II}=\mathbf{3 , 5}
\end{array}\right\}=3,5 \% \text { O }
$$

A. Differentialmanometer $\mathrm{I}$ bei $20^{\circ}$ im Wasserbade.

In die Birne ca. $0,1 \mathrm{ccm}$ Blut.

In die linke Birne In die rechte Birne

das gesättigte Blut das zu untersuch. Blut Differenz

Gleichgewicht bei . . . . .

$\begin{array}{lll}7,65 & 7,60 & \\ 7,22 & 8,10 & 0,93\end{array}$

Nach dem Schütteln

8,60

6,70

Hähne vorher geöffnet

B. Differentialmanometer II bei $20^{\circ} \mathrm{im}$ Wasserbade.

In die Birne ca. $0,1 \mathrm{ccm}$ Blut.

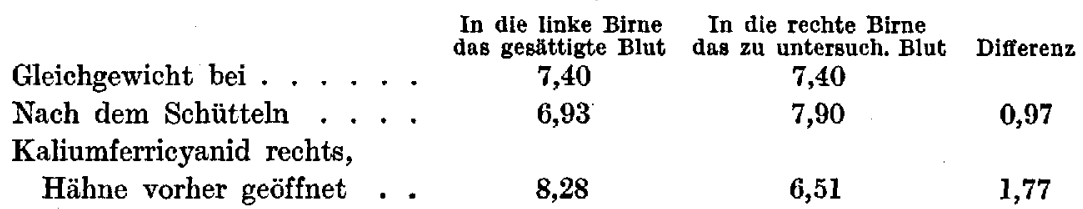

$$
\begin{aligned}
& \left.\% \text { Sättigung } \begin{array}{l}
\mathrm{A}=50,0 \\
\mathrm{~B}=45,2
\end{array}\right\}=47,6 \% \mathrm{O}_{2} \\
& \mathrm{O}_{2} \text {-Druck . . . . }=26,25 \mathrm{~mm} \mathrm{Hg} \\
& \mathbf{P}_{\mathrm{H}} \text {........ }=7,85 \text {. }
\end{aligned}
$$

Punkt II.

$2,0 \mathrm{ecm}$ Herzblut im Tonometer, daselbst ununterbrochen $25 \mathrm{Min}$. bei $38^{\circ}$ geschüttelt.

Temp.: $21^{\circ}$

Druck: $750 \mathrm{~mm} \mathrm{Hg}$ $\left.\begin{array}{lr}\text { Gasanalysen } & \mathrm{I}=5,7 \\ \text { aus dem Tonometer } & I I=5,7\end{array}\right\}=5,7 \% \mathrm{O}_{2}$

A. Differentialmanometer I bei $20^{\circ}$ im Wasserbade. In die Birne ca. 0,1 ccm Blut.

Gleichgewicht bei . . . . .

Nach dem Schütteln ....

Kaliumferricyanid rechts,

Hähne vorher nicht geöffnet
In die linke Birne In die rechte. Birne das gesättigte Blut das zu untersuch. Blut Differenz

$\begin{array}{lll}7,40 & 7,40 & \\ 7,23 & 7,61 & 0,38\end{array}$

8,49

6,33

2,16 
B. Differentialmanometer II bei $20^{\circ}$ im Wasserbade.

In die Birne ca. $0,1 \mathrm{com}$ Blut.

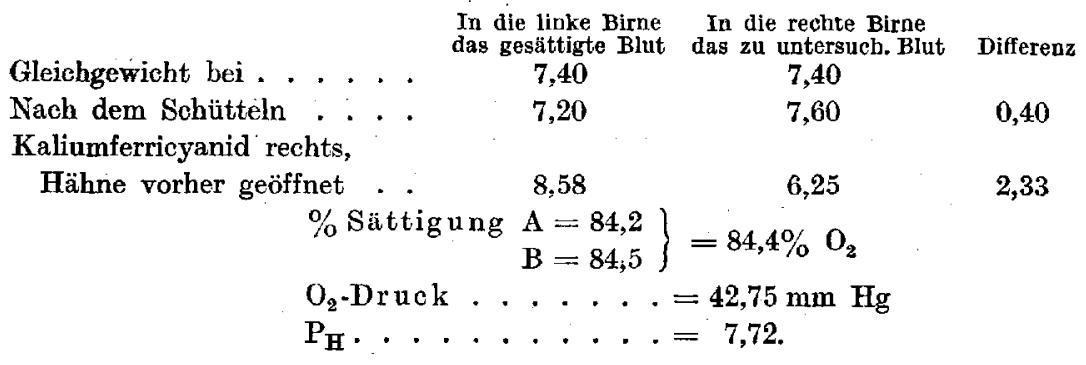

Vereinigt man diese Punkte zu einer Dissoziationskurve, so entsteht eine Linie, die einen verhältnismäßig hohen Verlauf zeigt.

\section{Fall Nr. 90.}

Maier, Helene, $3^{1} / 2$ Mon. Chronische Bronchopneumonie bei atrophischem Kinde. Geb. am.24. IV., ausgetragen, Geburtsgewicht unbekannt, von Anfang an künstlich ernährt, und zwar da. $21 / 2$ Mon. lang mit $1 / 3$-Milch mit etwas Zucker. Bekam dann etwas dünne Stühle, deswegen wurde die Milch fortgelassen. Zuerst 2 Wochen lang nur Haferschleim, dann die letzten 2 Wochen bis heute (10. VIII. 14) nur Reisschleim. Weil die Stühle immer noch nicht, gut geworden", bringt die Mutter das Kind in die Klinik. Hat in der letzten Zeit viel gehustet. Status: Hochgradig abgemagertes, leicht spastisches Kind mit grauer trockener Haut, über die beiden Lungenunterlappen bronchiales Atmen mit grobem. Geräusch und deutlicher Dämpfung. Sensorium frei. Schlaff und schwach. Das Kind bekommt Frauenmilch 250-500 g tăglich, die Stühle bleiben dabei etwas zerfahren, das Kind nimmt ständig zu. Ödeme nicht sichtbar. Bei der Aufnahme $2300 \mathrm{~g}$, beim Tode (2. IX.) $2670 \mathrm{~g}$ Gewicht. Die Dämpfungen bleiben bestehen, das Kind fiebert ständig von $37,5^{\circ}$ bis $39,5^{\circ}$. Am 30. IX. tritt diffuses Rasseln und Atemnot ein; und unter langsam zunehmender Herzschwäche stirbt das Kind am 3. IX.

Es wurden folgende Untersuchungen vorgenommen:

14. VIII. Sinuspunktion, dabei lebhaftes Schreien.

Blut, aktuelle Reaktion . . . . . . . 7,39

Blutkörperchen, gewaschen, in dest. $\mathrm{H}_{2} \mathrm{O}$ gelöst 6,73

3. IX. $6^{\mathrm{h}}$ vorm. Herzpunktion, gleich nach dem Exitus.

$$
\left.\begin{array}{c}
\text { Blut, aktuelle Reaktion . . . . . . . . . . . . . . . } 6,69 \\
\text { " } \mathrm{CO}_{2} \text {-freies . . . . } 7,57
\end{array}\right\}=88 \%
$$

Außerdem wurde die $\mathrm{O}_{2}$-Dissoziationskurve desselben Herzblutes wie folgt bestimmt:

$$
\begin{gathered}
\mathrm{O}_{2} \text {-Dissoziationskurve des Hämoglobins. } \\
\text { Punkt I. }
\end{gathered}
$$

2,0 ccm Herzblut im Tonometer, daselbst ununterbrochen $25 \mathrm{Min}$. bei $38^{\circ}$ geschüttelt.

Temp.: $20^{\circ}$

Druck: $752 \mathrm{~mm} \mathrm{Hg}$

$$
\left.\begin{array}{lr}
\text { Gasanalysen } & \mathrm{I}=4,6 \\
\text { aus dem Tonometer } & \mathrm{II}=4,4
\end{array}\right\}=4,5 \% \mathrm{O}_{2}
$$


A. Differentialmanometer I bei $18^{\circ}$ im Wasserbade.

In die Birne ca. 0,1 ccm Blut.

$\begin{array}{lcccc} & & \begin{array}{c}\text { In die linke Birne } \\ \text { das gesättgte Blnt }\end{array} & \begin{array}{c}\text { In die rechte Birne } \\ \text { das zu untersuch. Blut }\end{array} & \text { Differenz } \\ \text { Gleichgewicht bei . . . . . } & 7,40 & 7,40 & \\ \text { Nach dem Schütteln } & . . . & 7,32 & 7,49 & 0,17 \\ \text { Kaliumferricyanid rechts, } & . & & \\ \text { Hähno vorher nicht geöffnet } & 7,85 & 6,95 & 0,90\end{array}$

B. Differentialmanometer II bei $18^{\circ} \mathrm{im}$ Wasserbade.

In die Birne ca. $0,1 \mathrm{ccm}$ Blut.

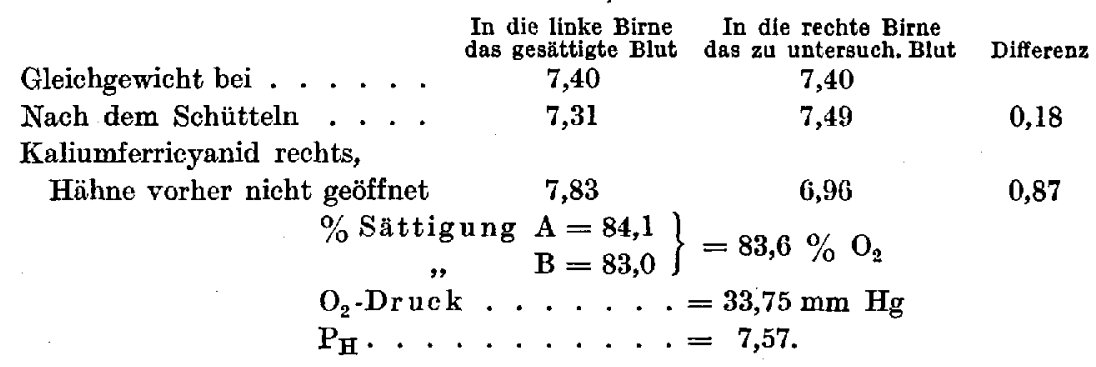

Punkt II.

$3,0 \mathrm{ccm}$ Herzblut im Tonometer, daselbst ununterbrochen 25 Min. bei $37,8^{\circ}$ geschüttelt.

Temp.: $20^{\circ}$

Druck: $752 \mathrm{~mm} \mathrm{Hg}$

$\left.\begin{array}{lr}\text { Gasanalysen } & \mathrm{I}=3,0 \\ \text { aus dem Tonometer } & \mathrm{II}=3,2\end{array}\right\}=3,1 \% \mathrm{O}_{2}$

Differentialmanometer bei $18^{\circ}$ im Wasserbade.

In die Birne ca. 0,1 ccm Blut.

Gleichgewicht bei .....

In die linke Birne In die rechte Birne

das geaăttigte Blut das zu untersuch. Blut Dlfferenz

Nach dem Schütteln ....

$7,63 \quad 7,60$

$\mathbf{7 , 4 9} \quad \mathbf{7 , 8 2}$

0,36

Kaliumferricyanid rechts,

.Hähne vorher nicht geöffnet

8,12

7,20

0,89

$$
\begin{aligned}
& \% \text { Sättigung }=71,2 \% \mathrm{O}_{2} \\
& \mathrm{O}_{2} \text {-Druck }=24,25 \mathrm{~mm} \mathrm{Hg} \\
& \mathrm{P}_{\mathrm{B}} \text { siehe Punkt } \mathrm{I} .
\end{aligned}
$$

Punkt III.

2,0 ecm Herzblut im Tonometer, daselbst ununterbrochen 25 Min. bei $38^{\circ}$ geschüttelt.

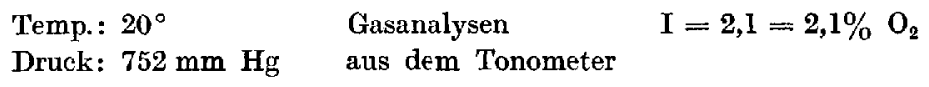

A. Differentialmanometer I bei $19^{\circ}$ im Wasserbade.

In die Birne ca. $0,1 \mathrm{ccm}$ Blut.

In die linke Birne In die rechte Birne

das gesāttigte Blut das $z u$ untersuch. Blut Differenz

Gleichgewicht bei . . . . $7,65 \quad 7,60$

Zeitschrift für Kinderheilkunde. O. XIV. 
448 Arvo Ylppö: Neugeborenen-, Hunger- und Intoxikationsacidosis.

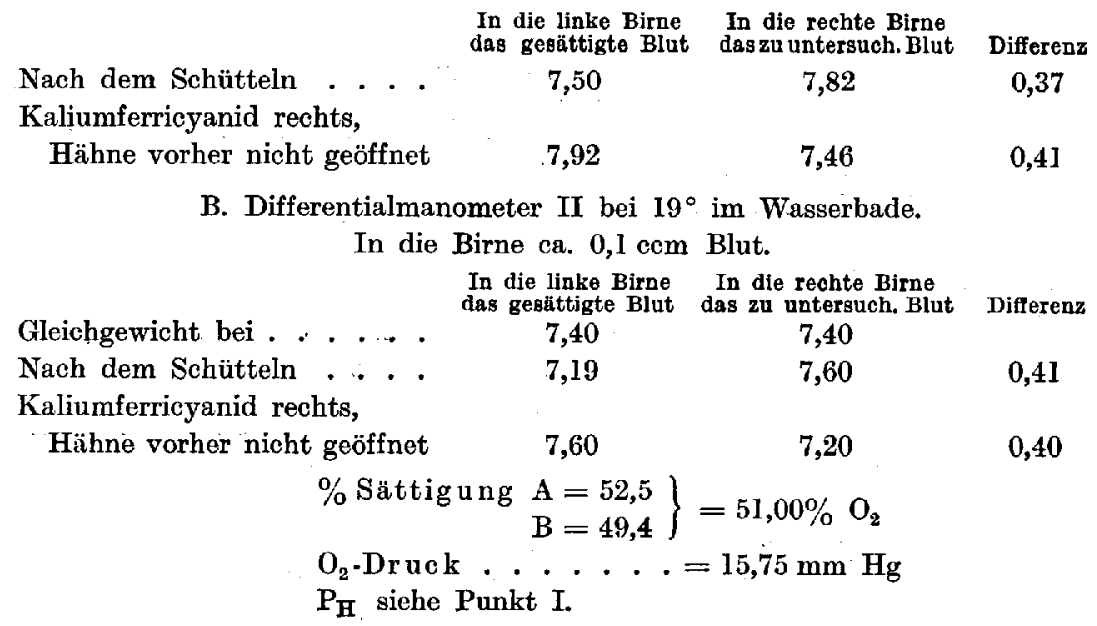

Die aus den obigen Zahlen gebaute Dissoziationskurve ist auf Fig. 24, I, S. 330, graphisch dargestellt. 